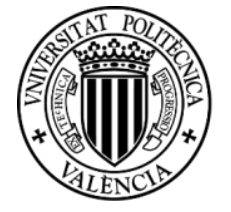

UNIVERSITAT
POLITĖCNICA
DE VALĖNCIA

\title{
Development and validation of a multi-scale and multi-physics methodology for the safety analysis of fast transients in Light Water Reactors
}

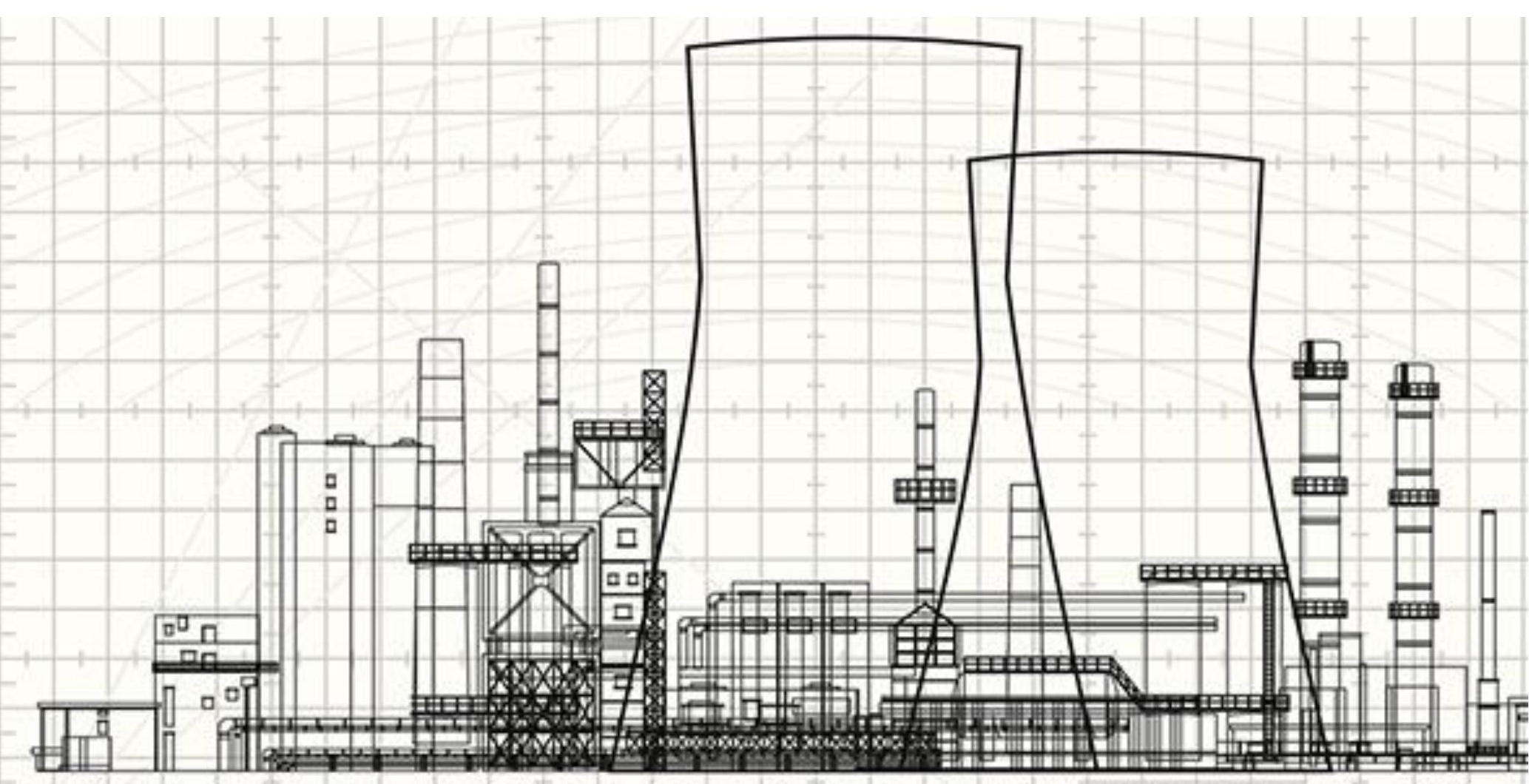

Ph.D. Thesis

Submitted by:

Patricio Hidalga García-Bermejo

Supervised by:

Dr. Rafael Miró Herrero

Dr. Gumersindo Jesús Verdú Martín 
UNIVERSITAT

POLITÈCNICA

DE VALÈNCIA

\section{Development and validation of a multi-scale and multi-physics methodology for the safety analysis of fast transients in Light Water Reactors}

Ph.D. Thesis

Submitted by:

Patricio Hidalga García-Bermejo

Supervised by:

Dr. Rafael Miró Herrero

Dr. Gumersindo Jesús Verdú Martín

October 2020 


\section{Acknowledgments}

I would like to thank first of all my thesis supervisors, Prof. Dr. Rafael Miró and Prof. Dr. Gumersindo Verdú for counting on me for the opportunity of developing this thesis work. This acknowledgment is extended to Kernkraftwerk Leibstadt and specially STA group, whose financial and technical support have made possible this research work. A special acknowledgement is addressed to Dr. Agustín Abarca, who has been not only a professional colleague, but also a de facto supervisor and mentor, even when he did not have to. Without him, I would not have been able to develop my current knowledge and skills in this scientific field that is the Nuclear Technology.

My personal acknowledgment goes to my fellow researchers at the ISIRYM and Department of Chemical and Nuclear Engineering of the UPV, who made the daily work less stressful than it could have been.

Finally, I have to thank the most to my parents, sisters, and my friends, the ones who were there back then, and of course the ones that still stand by me nowadays, despite the distance. Somehow and sometime you have been always there to give me your support, your trust and your encouragement, even in the toughest moments. You all helped to bring me to the final step of this journey. From the bottom of my heart: Thank you. 


\begin{abstract}
The nuclear technology for civil use has generated more concerns for the safety than several other technologies applied to the daily life. The Nuclear Regulators define the basis of how the Safety Operation of Nuclear Power Plants is to be done. According to these guidelines, a Nuclear Power Plant must analyze an envelope of hypothetical events and deterministically define if the acceptance criteria for these events is met. The Deterministic Safety Analysis uses simulation tools that apply the physics known in the behavior of the Nuclear Power Plant to evaluate the evolution of a safety variable and assure that the safety limits will not be exceeded.
\end{abstract}

The development of the computer science, the numerical methods and the physics involved in the behavior of a Nuclear Power Plant have yield powerful simulation tools that are capable to predict the evolution of safety variables which significant accuracy. This allows to consider more realistic simulation scenarios instead of conservative approaches in order to compensate the lack of knowledge in the applied prediction methods. The so called Best Estimate simulation tools are capable to analyze the transient events in different scales. Furthermore, they account more detailed analytical models and experimental correlations. A step forward in the Deterministic Safety Analysis intends to combine the Best Estimate simulation tools of the different physics considering the interaction among them and analyzing the different scales, considering more local approaches if necessary.

For this purpose, this thesis work presents a multi-scale and multi-physics methodology that uses different physics codes and has the aim of modeling postulated scenarios in different scales, i.e. from system models representing the components of the plants to the subchannel models that analyze the behavior of the coolant between the fuel rods. This methodology allows a flow of information where the output of one scale is used as input in a more detailed scale to predict a more local analysis of parameters, such as the Critical Power Ratio, which are of great importance for the estimation of safety margins. The development of this methodology has been validated against plant data with the aim of evaluating the scope of this methodology and in order to provide future lines of development. In addition, different results of the validation and verification yielded in the development of the parts of this methodology are presented. 


\section{Resumen}

La tecnología nuclear para el uso civil genera más preocupación por la seguridad que muchas otras tecnologías que se usan a diario. La Autoridad Nuclear define las bases de cómo debe realizarse la operación segura de una Central Nuclear. De acuerdo a las directrices establecidas por la Autoridad Nuclear, una Central Nuclear debe analizar una envolvente de escenarios hipotéticos y comprobar de manera determinista que los criterios de aceptación para dicho evento se cumplen. El Análisis Determinista de Seguridad utiliza herramientas de simulación que aplican la física conocida sobre el comportamiento de la Central Nuclear para evaluar la evolución de una variable de seguridad y asegurar que los límites no se sobrepasan.

El desarrollo de la tecnología informática, de los métodos matemáticos y de la física que envuelve el comportamiento de una Central Nuclear han proporcionado herramientas de simulación potentes que son capaces de predecir el comportamiento de las variables de seguridad con una importante precisión. Esto permite analizar escenarios de manera más realista evitando asumir condiciones conservadoras que hasta la fecha compensaban la falta de conocimiento modelado en las herramientas de simulación. Las herramientas conocidas como De Mejor Estimación son capaces de analizar eventos transitorios en diferentes escalas. Además, emplean modelos analíticos de las diferentes físicas más detallados, así como correlaciones experimentales más realistas y actuales. Un paso adelante en el Análisis Determinista de Seguridad pretende combinar las diferentes herramientas de Mejor Estimación que se emplean para analizar las distintas físicas de una Central Nuclear, considerando incluso la interacción entre ellas y el análisis progresivo a diferentes escalas, llegando a analizar fenómenos más locales si es necesario.

Para este fin, esta tesis presenta una metodología de análisis multi-físico y multi-escala que emplea diferentes códigos de simulación analizando el escenario propuesto a diferentes escalas, es decir, desde un nivel de planta que incluye los distintos componentes, hasta el volumen de control que supone el refrigerante pasando entre las varillas de combustible. Esta metodología permite un flujo de información que va desde el análisis a mayor escala hasta el de menor escala. El desarrollo de esta metodología ha sido validado con datos de planta para poder evaluar el alcance de esta metodología y proporcionar nuevas líneas de trabajo futuro. Además, se han añadido los resultados de los distintos procesos de validación y verificación que han surgido a lo largo de este trabajo. 


\section{Resum}

La tecnologia nuclear per a l'ús civil genera més preocupació per la seguretat que moltes altres tecnologies d'ús quotidià. L'Autoritat Nuclear defineix les bases de com ha de realitzar-se l'operació segura d'una Central Nuclear. D'acord amb les directrius establertes per l'Autoritat Nuclear, una Central Nuclear ha d'analitzar una envoltant d'escenaris hipotètics I comprovar de manera determinista que els criteris d'acceptació per a l'esdeveniment seleccionat es compleixen. L'Anàlisi Determinista de Seguretat utilitza eines de simulació que apliquen la física coneguda sobre el comportament de la Central Nuclear per avaluar l'evolució d'una variable de seguretat i assegurar que els límits no es traspassen.

El desenvolupament de la tecnologia informàtica, els mètodes matemàtics i de la física que envolta el comportament d'una Central Nuclear han proporcionat eines de simulació potents amb capacitat de predir el comportament de les variables de seguretat amb una precisió significativa. Això permet analitzar escenaris de manera realista evitant assumir condicions conservadores que fins al moment compensaven la mancança de coneixement. Les eines de simulació conegudes com De Millor Estimació son capaces d'analitzar esdeveniment transitoris a diferent escales. A més, utilitzen models analítics per a les diferents físiques amb més detall així com correlacions experimentals més actualitzades i realistes. Un pas més endavant en l'Anàlisi Determinista de Seguretat pretén combinar les diferents eines de Millor Estimació que se utilitzen per analitzar les distintes físiques d'una Central Nuclear, considerant inclús la interacció entre ells i l'anàlisi progressiu a diferents escales, amb la finalitat de poder analitzar fenòmens locals.

Per a aquest fi, esta tesi presenta una metodologia d'anàlisi multi-física i multi-escala que utilitza diferents codis de simulació analitzant l'escenari proposat a diferents escales, és a dir, des d'un nivell de planta que inclou els distints components, fins al volum de control que suposa el refrigerant passant entre les varetes de combustible. Esta metodologia permet un flux de informació que va des de l'anàlisi d'una escala major a una menor. El desenvolupament d'aquesta metodologia ha sigut validada i verificada amb dades de planta $\mathrm{i}$ els resultats han sigut analitzats a fi d'avaluar la capacitat de la metodologia i les possibles línies de treball futur. A més s'han afegit els principals resultats de verificació i validació que han sorgit en les distintes etapes d'aquest treball. 


\section{INDEX OF CONTENTS}

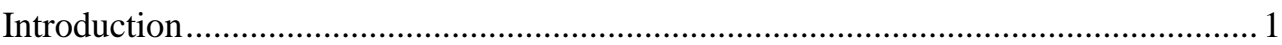

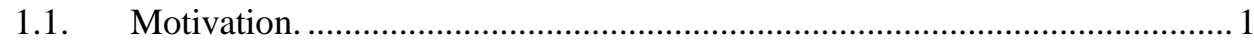

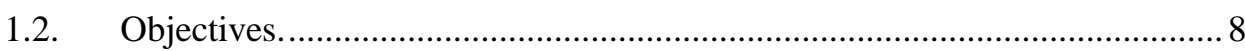

1.3. Organization of the contents of the thesis ................................................. 8

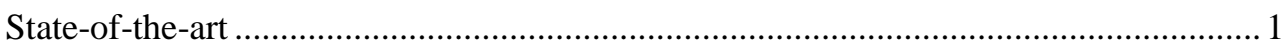

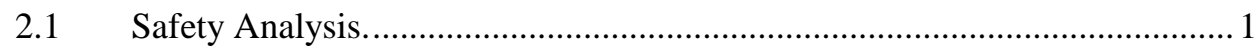

2.2. Safety Analysis in a Nuclear Power Plant................................................ 5

2.3. Transient events in Light Water Reactors................................................. 8

2.4. Classification of the events in a Nuclear Power Plant with LWRs. ...............9

2.4.1. Classification according to probability of the Initiating Event. ............. 9

2.4.2. Classification according to variable change........................................... 11

2.5. The use of simulation tools for the Safety Evaluation.................................. 14

2.5.1. Surrogated variables used in the Deterministic Safety Analysis.......... 16

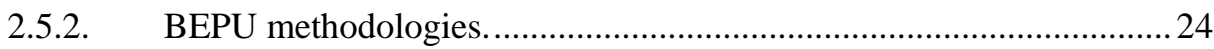

2.6. The role of Sensitivity and Uncertainty Analysis........................................28

2.7. Multi-physics and multi-scale features....................................................... 41

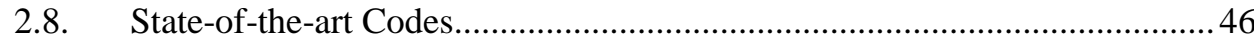

2.8.1. Neutron transport codes. ................................................................. 46

2.8.2. Thermal-Hydraulic codes at System Level. ......................................... 48

2.8.3. Thermal-Hydraulic codes at Subchannel Level. .....................................51

2.8.4. Coupled Features: Thermal-Hydraulics and Neutron Kinetics............51

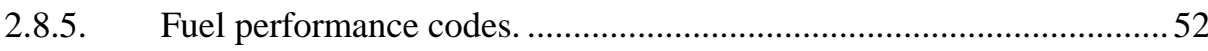

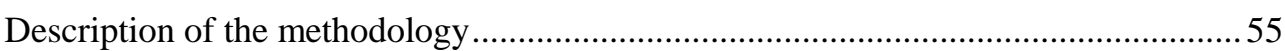

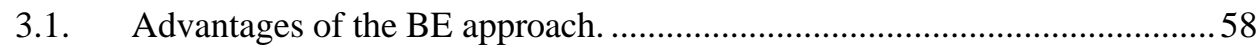

3.2. Relevant steps of a BE simulation methodology.........................................61

3.3. Features of the methodology and the selected simulation tools...................66

3.3.1. Generation of cross-section libraries....................................................66

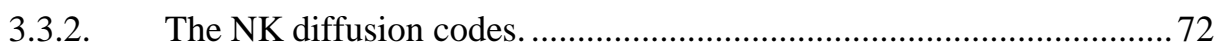

3.3.3. Simulation of system models: System codes and NK coupling............72 
3.3.4. Coupled TH/NK subchannel core simulation........................................74

3.3.5. TH subchannel fuel assembly simulation..............................................75

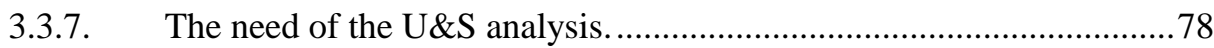

3.4. Examples that summarize the application of the methodology to a real case. 79

Validation and Verification of codes and methodologies for two application cases.....82

4.1. Code and Methodology qualification for safety analysis. .83

4.2. Methodology for the generation of Homogenized 2-Group Cross Section Libraries. . .84

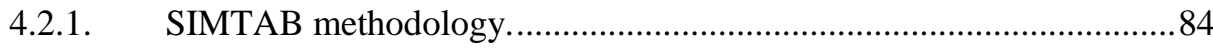

4.2.2. Developed work on GenPMAXS methodology..................................... 85

4.2.3. Assessed sources of errors in GenPMAXS development......................87

4.2.4. Current results with GenPMAXS methodology....................................91

4.2.5. Conclusions and future work for GenPMAXS methodology..............100

4.3. Validation of void fraction correlation in CTF-UPVIS against the PSBT

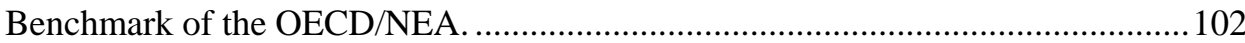

4.3.1. Description of the validated methodology. ..........................................103

4.3.2. PSBT Benchmark of the OECD/NEA................................................. 105

4.3.3. CTF-UPVIS simulation model............................................................ 109

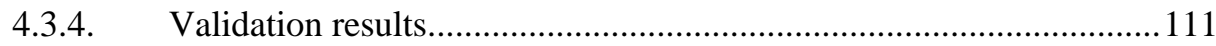

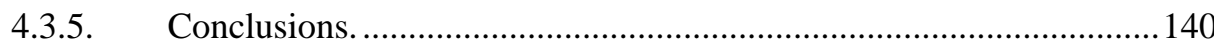

Application of the proposed methodology to the Safety Analysis of the Turbine Trip

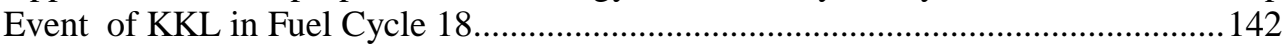

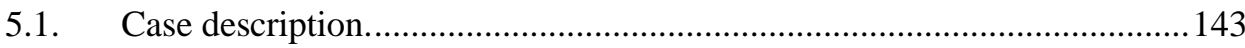

5.2. Road map of the application of the methodology........................................149

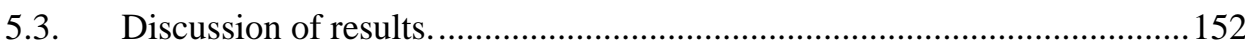

5.3.1. Verification of the cross-section libraries...........................................152

5.3.2. Simulation of the coupled thermal-hydraulic and neutron kinetic system model. 156

5.3.3. Simulation of the coupled TH and NK core model defined channel-bychannel. 161

5.3.4. Simulation of the subchannel thermal-hydraulic model .165 
5.3.5. Pin analysis including the fuel behavior simulation............................ 170

5.3.6. Uncertainty and sensitivity analysis................................................ 172

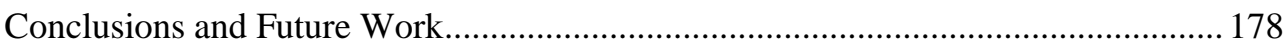

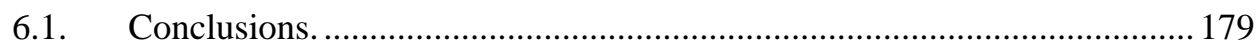

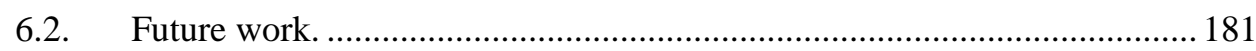

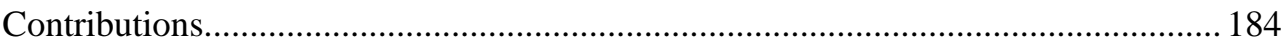

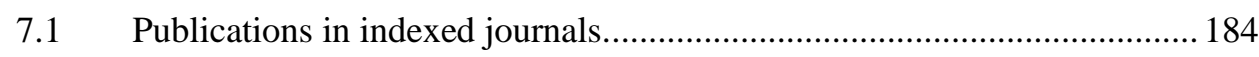

7.2. Oral participation in International Conferences........................................ 185

7.3. Oral presentations in national conferences................................................ 188

7.4. Development of projects in the framework of collaboration with different private companies that generated a series of technical reports. ............................... 189

7.5. Technical reports produced in the framework of the Thesis Project ........... 191

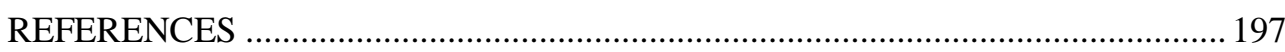




\section{INDEX OF FIGURES}

Figure 2.1 Scheme of the physical barriers containing the radioactive material. ................ 2

Figure 2.2 Phenomenology of the fuel cladding temperature increase beyond the PCT

(NEA, 2009). . .18

Figure 2.3 Behavior of the heat flux as a function of temperature difference between cladding surface and saturated coolant (NEA, 2009)

Figure 2.4 Simplified scheme of pellet-cladding system prior operation (left), irradiated and prior to $\mathrm{PCl}$ (middle) irradiated and after $\mathrm{PCl}$ (right).

Figure 2.5 Scheme of the approaches to the safety margins assessment. ......................... 26

Figure 2.6 Uncertainty flow through the code simulation. ........................................... 28

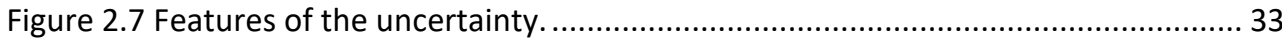

Figure 2.8 System scale modelled with SNAP tool for the simulation with TRACE code. ... 42

Figure 2.9 3D core modelled channel-by-channel represented with Paraview with the fuel channel layout.

Figure 2.10 Axial and radial nodal distribution for a fuel assembly modelled for COBRA-TF.

Figure 2.11 Fuel rod model for the thermo-mechanical analysis for FRAPTRAN code. ...... 44

Figure 3.1 Diagram of the multi-scale feature of the proposed methodology. .................. 62

Figure 3.2 Diagram of the composition of the channel-by-channel core from the pin-by-pin fuel models.

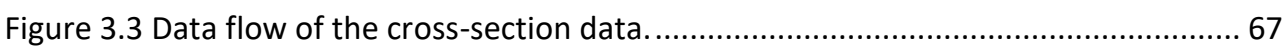

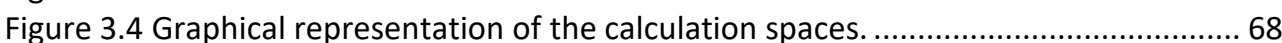

Figure 3.5 Nodal distribution of a BWR for a Neutron Diffusion 3D code............................. 72

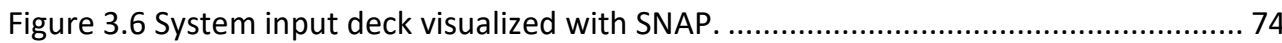

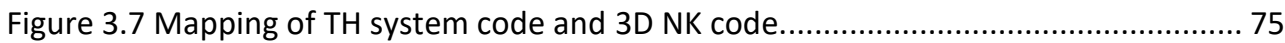

Figure 3.8 Heat Transfer scheme of the fuel rod.......................................................... 77

Figure 4.1 Approach of the built-in Branch Structure in CASMO ........................................ 86

Figure 4.2 Adapted Branch Structure needed for GenPMAXS when reading CASMO output files.

Figure 4.3 Mismatch in the nodal distribution and the regions of the different CrossSection Libraries. 90

Figure 4.4 Data flow for the generation and verification of Cross Section Libraries.......... 93

Figure 4.5 Core distribution in geometrical nodes (left) and in segments (right). ............. 94

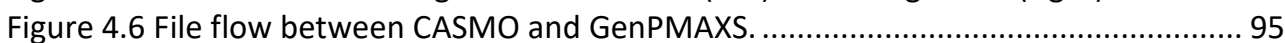

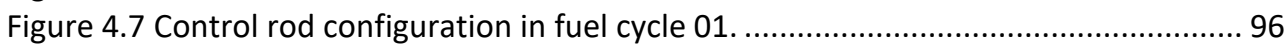

Figure 4.8 Axial power profile comparison: PMAXS/PARCS vs CASMO4/SIMULATE3 (C01EOC). 97

Figure 4.9 Radial error distribution (\%) of the power comparison: PMAXS/PARCS vs CASMO4/SIMULATE3 (C01EOC).

Figure 4.10 Axial power profile comparison: PMAXS/PARCS vS CASMO4/SIMULATE3 (CO1EOC-ARO). 
Figure 4.11 Radial error distribution (\%) of the power comparison: PMAXS/PARCS vs

CASMO4/SIMULATE3 (C01EOC-ARO)................................................................ 100

Figure 4.12 Graphical scheme of the test facility of the PSBT Benchmark (Rubin, 2010)..106

Figure 4.13 Evolution of the inlet coolant enthalpy (left) and mass flow (right).............107

Figure 4.14 Evolution of the outlet coolant pressure (left) and the power (right). ...........107

Figure 4.15 Layout of the rod array of the PSBT test facility. ............................................ 109

Figure 4.16 Validation of the prediction of void fraction against measurement of the benchmark for the measurement point 1 (2.216 $\mathrm{m})$.

Figure 4.17 Validation of the prediction of void fraction against measurement of the benchmark for the measurement point $2(2.669 \mathrm{~m})$

Figure 4.18 Validation of the prediction of void fraction against measurement of the benchmark for the measurement point $3(3.177 \mathrm{~m})$.............................................. 114

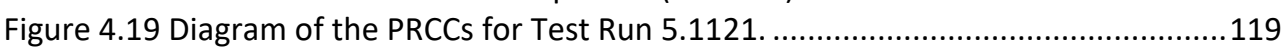

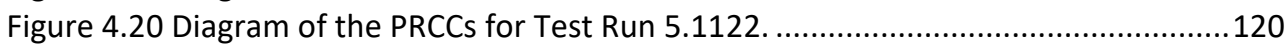

Figure 4.21 Diagram of the PRCCs for Test Run 5.2111.............................................121

Figure 4.22 Diagram of the PRCCs for Test Run 5.2112 ............................................122

Figure 4.23 Diagram of the PRCCs for Test Run 5.2442 .............................................123

Figure 4.24 Diagram of the PRCCs for Test Run 5.3441............................................124

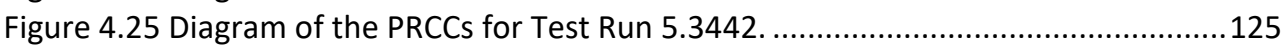

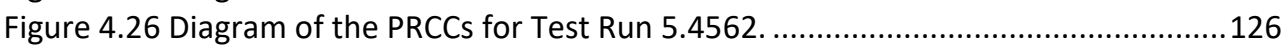

Figure 4.27 Diagram of the PRCCs for Test Run 5.6321............................................127

Figure 4.28 Diagram of the PRCCs for Test Run 5.6322 ............................................128

Figure 4.29 Diagram of the PRCCs for Test Run 5.6552 ............................................129

Figure 4.30 Evolution of the void fraction and statistical parameters for the lower measurement point of the PSBT case............................................................... 131

Figure 4.31 Evolution of the void fraction and statistical parameters for middle measurement point of the PSBT case................................................................... 132

Figure 4.32 Evolution of the void fraction and statistical parameters for the upper measurement point of the PSBT case.

Figure 4.33 Comparison against the results of other PBST participants for the Lower Measurement Point.

Figure 4.34 Comparison against the results of other PBST participants for the Lower Measurement Point........................................................................................... 135

Figure 4.35 Comparison against the results of other PBST participants for the Lower Measurement Point.

Figure 4.36 Sensitivity Analysis of the transient conditions for the Lower Measurement Point.

Figure 4.37 Sensitivity Analysis of the transient conditions for the Lower Measurement Point.

Figure 4.38 Sensitivity Analysis of the transient conditions for the Lower Measurement Point.

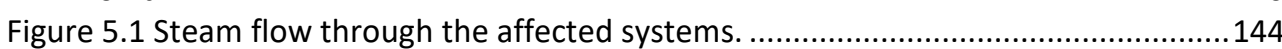


Figure 5.2 TCVs movement during the transient event.

Figure 5.3 TBVs movement during the transient event.

Figure 5.4 Scheme of information flow through the different steps of the proposed methodology.

Figure 5.5 Nodal distribution of the 3D core model for PARCS....................................... 153

Figure 5.6 Comparison of the axial power profile of PARCS-NEMTAB and SIMULATE-3. . 154

Figure 5.7 Radial distribution of the relative error (\%) of the comparison PARCS (NEMTAB) vs SIMULATE-3

Figure 5.8 TRACE model scheme represented with SNAP.......................................... 157

Figure 5.9 Total core power evolution: TRACE/PARCS(NEMTAB) vs Plant Data. .............. 159

Figure 5.10 Dome pressure evolution: TRACE/PARCS vs Plant Data.............................. 160

Figure 5.11 Reactor core evolution of the CTF-UPVIS/PARCS model and TRACE/PARCS model against plant data.

Figure 5.12 Evolution of the Minimum CPR of the fuel critical fuel channels retrieved from the simulation of the CTF-UPVIS/PARCS core model.

Figure 5.13 core map highlighting the critical fuel channels according to the Minimum CPR.

Figure 5.14 Radial nodal distribution of fuel channel 509 166

Figure 5.15 Axial nodal distribution of the fuel channel 509 with the detail of the partial length rods.

Figure 5.16 Comparison of the prediction of the Minimum CPR between the fuel channel 509 of the CTF-UPVIS/PARCS core model and the average pin-by-pin fuel model of CTF-UPVIS

Figure 5.17 Evolution of the Minimum CPR with the approaches of the average value, the envelope and the critical fuel rod.

Figure 5.18 Prediction of the Minimum CPR by FRAPCON/FRAPTRAN pin model compared with the prediction of the CTF-UPVIS pin model. 172

Figure 5.19 Statistics results of the Minimum CPR prediction with FRAPTRAN pin model.

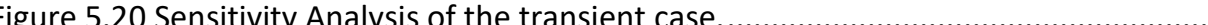




\section{INDEX OF TABLES}

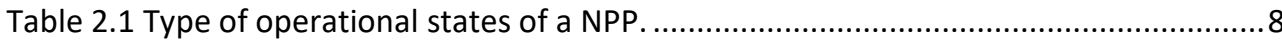

Table 2.2 Transient cases for BWRs and PWRs........................................................... 10

Table 2.3 (Continued) Transient cases for BWRs and PWRs..........................................11

Table 2.4 Transient events in LWRs categorized as Postulated Accidents. .......................... 11

Table 2.5 Different approaches for the Deterministic Safety Analysis. ...............................25

Table 2.6 Differences between stochastic and epistemic uncertainty. ...............................32

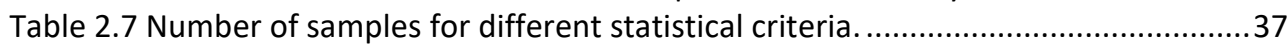

Table 3.1 Classification of occurrences in a NPP........................................................56

Table 3.2 Options for the different combinations regarding the code accuracy and the ac-

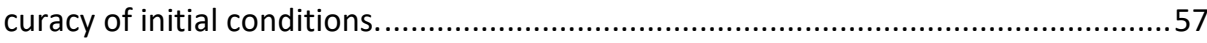

Table 4.1 History and branch structure in a regular distribution done by CASMO..............88

Table 4.2 History structure in an adequate distribution for GenPMAXS. ..........................89

Table 4.3 Branch structure in an adequate distribution for GenPMAXS. ...........................89

Table 4.4 Summary of comparison results for case EOC01: PMAXS/PARCS vs CASMO4/SIM-

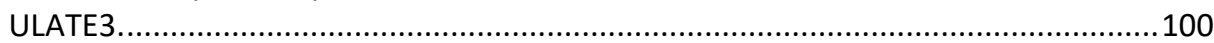

Table 4.5 File size and computational time needed for fuel cycle calculations with

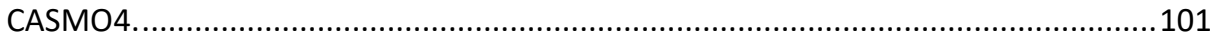

Table 4.6 Uncertainty definition of the input variables for the PSBT simulation. ..............105

Table 4.7 Boundary conditions for the simulation models. .............................................108

Table 4.8 Nodal distribution in the axial length of the PSBT model for CTF-UPVIS. ..........110

Table 4.9 Design parameters of the test facility. ............................................................. 111

Table 4.10 Absolute error of the comparison between CTF prediction and measured value

and test boundary conditions......................................................................... 115

Table 4.11 Absolute error of the comparison between CTF prediction and measured value

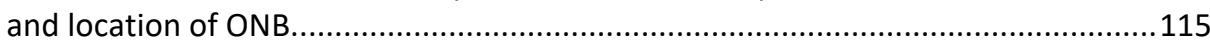

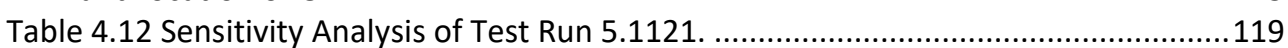

Table 4.13 Sensitivity Analysis of Test Run 5.1222. ...........................................................119

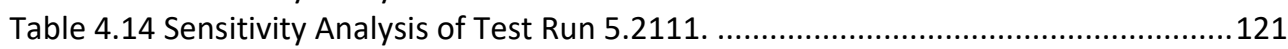

Table 4.15 Sensitivity Analysis of Test Run 5. 2112. ....................................................122

Table 4.16 Sensitivity Analysis of Test Run 5.2442. ......................................................123

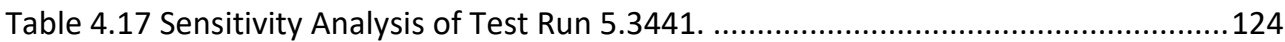

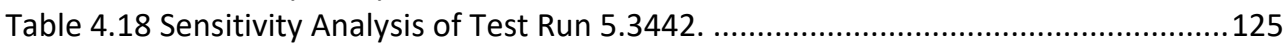

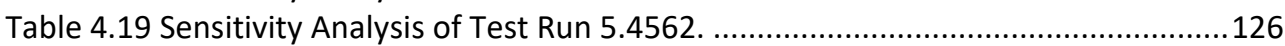

Table 4.20 Sensitivity Analysis of Test Run 5.6321. ........................................................127

Table 4.21 Sensitivity Analysis of Test Run 5.6322. ....................................................... 128

Table 4.22 Sensitivity Analysis of Test Run 5.6552 ......................................................129

Table 5.1 Turbine Trip Fuel Cycle 18 sequence of events................................................148

Table 5.2 Information of the simulation codes used in the application of the proposed methodology. ........................................................................................... 150

Table 5.3 Summary of the results of the comparison PARCS-NEMTAB vs SIMULATE-3 ....156 
Table 5.4 Summary of results of the code-to-code verification of TRACE/PARCS (NEMTAB)

vs SIMULATE-3. 158

Table 5.5 Summary of results of the code-to-code verification of CTF-UPVIS/PARCS

(NEMTAB) Vs SIMULATE-3.

161

Table 5.6 Minimum CPR for each of the fuel critical fuel channels.................................. 165

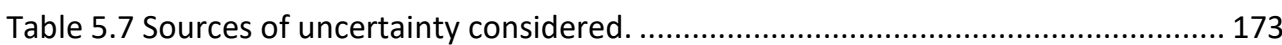

Table 5.8 Sensitivity Analysis of the transient case...................................................... 175 


\section{ACRONYMS}

\begin{tabular}{|c|c|}
\hline ANM & Analytical Nodal Method \\
\hline $\mathrm{AOO}$ & Anticipated Operational Occurrence \\
\hline APIs & Application Programming Interfaces \\
\hline ASM & Additive Schwarz Method \\
\hline ASME & American Society of Mechanical Engineers \\
\hline ATWS & Anticipated Transient Without SCRAM \\
\hline $\mathrm{BE}$ & Best Estimate \\
\hline BiCGSTAB & Biconjugate Gradient Stabilized Method \\
\hline $\mathrm{BOC}$ & Beginning Of Cycle \\
\hline BWRs & Boiling Water Reactors \\
\hline CAMP & Code Applications and Maintenance Program \\
\hline CASL & Consortium for Advanced Simulation of Light Water Reactors \\
\hline CDS & Central Difference Scheme \\
\hline CEA & Commissariat à l'Énergie Atomique \\
\hline CFD & Computational Fluid Dynamics \\
\hline CFR & Code of Federal Regulations \\
\hline CMFD & Coarse Mesh Finite Difference Method \\
\hline $\mathrm{CNCC}$ & Corrective Nodal Coupling Coefficient \\
\hline CPR & Critical Power Ratio \\
\hline $\mathrm{CR}$ & Control Rod \\
\hline CSNI & Committee on the Safety of Nuclear Installations \\
\hline CVCS & Control Volume Chemical System \\
\hline DNBR & Departure of Nucleate Boiling Ratio \\
\hline ECCS & Emergency Core Cooling System \\
\hline ECI & Exterior Communications Interface \\
\hline EPRI & Electric Power Research Institute, Inc \\
\hline
\end{tabular}




\begin{tabular}{|c|c|}
\hline ETIPs & European Technology and Innovation Platforms \\
\hline EURATOM & European Atomic Commission \\
\hline FEM & Finite Elements Method \\
\hline FGE & Forward Gaussian Elimination \\
\hline FMFD & Fine Mesh Finite Difference Method \\
\hline FOM & Figure Of Merit. \\
\hline FTCS & Forward Time/Central Space method \\
\hline FVM & Finite Volume Method \\
\hline GAMMA & CSNI Working Group on the Analysis and Management of Accidents \\
\hline GDC & General Design Criteria \\
\hline GE & General Electric \\
\hline GI & General Interface \\
\hline GRS & Gessellschaft für Anlagen-und Reaktorsicherheit \\
\hline HFP & Hot Full Power \\
\hline HPCS & High Pressure Core Spray \\
\hline HPIS & High Pressure Injection System \\
\hline HZDR & Helmholtz-Zentrum Dresden-Rossendorf \\
\hline $\mathrm{HZP}$ & Hot Zero Power \\
\hline IAEA & International Atomic Energy Agency \\
\hline ICE & Implicit Continuous Eulerian \\
\hline ILU & Incomplete LU Factorization \\
\hline INL & Idaho National Laboratory \\
\hline INSAG & International Nuclear Safety Group \\
\hline IRSN & French Nuclear Safety Institute \\
\hline KIT & Karlsruhe Institute of Technology \\
\hline LOCA & Loss of Coolant Accident \\
\hline LWR & Light Water Reactor \\
\hline MCPR & Minimum Critical Power Ration \\
\hline MLHGR & Maximum Linear Heat Generation Ratio \\
\hline
\end{tabular}




\begin{tabular}{ll} 
MPI & Message Passing Interface \\
MTU & Metric Ton of Uranium Dioxide \\
NEA & Nuclear Energy Agency \\
NEM & Nodal Expansion Method \\
NPP & Nuclear Power Plant \\
NULIFE & Nuclear Plant Life Prediction \\
NURESIM & European Platform for Nuclear Reactor Simulations \\
NURISP & Nuclear Reactor Integrated Simulation Project \\
NUSAFE & Nuclear Waste Management and Safety as well as Radiation Research \\
OECD & Organisation for Economic Co-operation and Development \\
PCMI & Pellet-Cladding Mechanical Interaction \\
PCT & Peak Cladding Temperature \\
PDD & Power Density Detector \\
PETSc & Portable, Extensible Toolkit for Scientific Computation \\
PPR & Pin Power Reconstruction \\
PVM & Parallel Virtual Machine \\
PWRs & Pressurized Water Reactors \\
QUICK & Quadratic Upwind Interpolation for Convective Kinematics \\
RCS & Reactor Coolant System \\
RIA & Reactivity Insertion Accident \\
RMSE & Root Mean Square Error \\
RPT & Recirculation Pump Trip \\
RSICC & Radiation Safety Information Computational Center \\
S3 & SIMULATE-3 \\
SALOME & The Open Source Integration Platform for Numerical Simulation \\
SET-Plan & European Strategic Energy Technology Plan \\
SETS & Stability Enhancing Two-Step \\
SIMPLE & Semi-Implicit Method for Pressure-Linked Equations \\
SNETP & Sustainable Nuclear Energy Technology Platform \\
\hline
\end{tabular}




$\begin{array}{ll}\text { SOR } & \text { Successive Over Relaxation } \\ \text { SP3 } & \text { Simplified P3 Approach. } \\ \text { SRP } & \text { Standard Review Plan } \\ \text { SRV } & \text { Safety/Relief Valve } \\ \text { TBV } & \text { Turbine Bypass Valve } \\ \text { TSV } & \text { Turbine Stop Valve } \\ \text { TT } & \text { Turbine Trip } \\ \text { UDS } & \text { Upwind Difference Scheme } \\ \text { EU } & \text { European Union } \\ \text { UPV } & \text { Universitat Politècnica de València } \\ \text { USNRC } & \text { United States Nuclear Regulatory Commission } \\ \text { V\&V } & \text { Validation and Verification } \\ \text { WNA } & \text { World Nuclear Association } \\ \text { 1-D } & \text { Unidimensional } \\ \text { 2-D } & \text { Bidimensional } \\ \text { 3-D } & \text { Tridimensional }\end{array}$




\section{NOMENCLATURE}

\begin{tabular}{|c|c|}
\hline$\alpha$ & Void Fraction (non-dimensional) \\
\hline$\beta_{k}$ & Deferred neutron precursor fraction of the k-group (non-dimensional) \\
\hline$\Sigma_{t, g}$ & Macroscopic transport cross section of energy group $g\left(\mathrm{~cm}^{-1}\right)$ \\
\hline$\Sigma_{S, g \rightarrow g \prime}$ & Macroscopic scattering cross section of energy group $g$ to $g^{\prime}\left(\mathrm{cm}^{-1}\right)$ \\
\hline$\Sigma_{f, g}$ & Macroscopic fission cross section of energy group $g\left(\mathrm{~cm}^{-1}\right)$ \\
\hline$\Sigma_{a, g}$ & Macroscopic absorption cross section of energy group $g\left(\mathrm{~cm}^{-1}\right)$ \\
\hline$\sigma_{k}$ & $\begin{array}{l}\text { Volumetric force tensor applied to the k-phase of a multiphase fluid } \\
\left(\mathrm{N} / \mathrm{m}^{3}\right)\end{array}$ \\
\hline$\lambda_{k}$ & Deferred neutron decay constant of the $k$ precursor group $\left(\mathrm{s}^{-1}\right)$ \\
\hline$\mu$ & Dynamic Viscosity (Pa-s) \\
\hline$v$ & Average Neutrons Emitted by fission (non-dimensional) \\
\hline$\rho$ & Density $\left(\mathrm{kg} / \mathrm{m}^{3}\right)$ \\
\hline$\rho_{k}$ & Density of the $k$-phase of a multiphase fluid $\left(\mathrm{kg} / \mathrm{m}^{3}\right)$ \\
\hline$\phi_{g}$ & Neutron Flux for energy group $g$ (neutrons $/ \mathrm{cm}^{2}$ ) \\
\hline$\chi_{g}$ & Fission spectrum of the neutrons of the energy group $g$ \\
\hline$A$ & Cross section area $\left(\mathrm{m}^{2}\right)$ \\
\hline$\vec{A}$ & Area Vector $\left(\mathrm{m}^{2}\right)$ \\
\hline$C_{D}$ & Drag coefficient (non-dimensional) \\
\hline$C_{f}$ & Specific Heat Capacity of the fuel pellet $(\mathrm{J} / \mathrm{kg}-\mathrm{K})$ \\
\hline$C_{k}$ & $\begin{array}{l}\text { Concentration of delayed neutron precursors of the } k \text {-group (neu- } \\
\text { trons } / \mathrm{cm}^{3} \text { ) }\end{array}$ \\
\hline$D_{g}$ & Neutron diffusion constant of the energy group $g\left(\mathrm{~cm}^{-1}\right)$ \\
\hline$E$ & Conservation of energy \\
\hline$g$ & Gravity acceleration (m/s²) \\
\hline$h_{f}$ & Fuel Pellet Enthalpy (J/kg). \\
\hline
\end{tabular}




\begin{tabular}{|c|c|}
\hline$h_{s a t, k}$ & Saturation Enthalpy the $k$-phase of a multiphase fluid $(\mathrm{J} / \mathrm{kg})$ \\
\hline$J_{g}$ & $\begin{array}{l}\text { Densidad de corriente neutrónica del grupo de energía } g \text { (neutro- } \\
\text { nes } / \mathrm{cm}^{2} \text { ) }\end{array}$ \\
\hline$k$ & Thermal Conductivity (J/m-s-K) \\
\hline$k_{e f f}$ & Neutron multiplication constant (non-dimensional) \\
\hline$M$ & Conservation of mass \\
\hline$\dot{m}$ & Mass Flow (kg/s) \\
\hline$\vec{n}$ & Unit vector normal to an area $\left(\mathrm{m}^{2}\right)$ \\
\hline$p$ & Pressure $(\mathrm{Pa})$ \\
\hline$Q$ & Heat per volume unit $\left(\mathrm{J} / \mathrm{m}^{3}\right)$ \\
\hline$\vec{r}$ & Vector position (m) \\
\hline$T$ & Temperature $(\mathrm{K})$ \\
\hline$T_{f}$ & Fuel pellet temperature $(\mathrm{K})$ \\
\hline$T_{0}$ & Initial temperature $(\mathrm{K})$ \\
\hline$t$ & Time (s) \\
\hline$U$ & Internal Energy per volume unit $\left(\mathrm{J} / \mathrm{m}^{3}\right)$ \\
\hline$u$ & Velocity Scalar Component parallel to axis $x(\mathrm{~m} / \mathrm{s})$ \\
\hline $\mathrm{V}$ & Volume $\left(\mathrm{m}^{3}\right)$ \\
\hline$v$ & Velocity Scalar Component parallel to axis $y(\mathrm{~m} / \mathrm{s})$ \\
\hline$v_{g}$ & Mean neutron velocity of the neutrons in the energy group $g(\mathrm{~cm} / \mathrm{s})$ \\
\hline$W$ & Work per volume unit $\left(\mathrm{J} / \mathrm{m}^{3}\right)$ \\
\hline$w$ & Velocity Scalar Component parallel to axis $z(\mathrm{~m} / \mathrm{s})$ \\
\hline
\end{tabular}

xviii 
To all the workers of the Scientific Research Field, that with his compromise struggle to make of this profession a dignified one. 



\section{Chapter 1 Introduction}

\subsection{Motivation.}

The field of the Nuclear Technology has been under constant supervision since its use as a conventional technology for civil use. One of the main uses focuses in the generation of electric energy and was defined by those states with the role of the avant-garde in the Nuclear Technology at the moment: The United States of America and the Soviet Union. On the one hand, it starts with the first Nuclear Power Plant (NPP) able of producing usable amounts of electricity in 1951 in the United States, the Chicago Pile-4 operated by Argonne National Laboratory. On the other hand, The AM-1 Obninsk was the first NPP connected to the grid for electricity supply in 1954 in the Soviet Union. This conventional and broad scale use of the Nuclear Technology was preceded by the corresponding establishment of the regulatory agencies in both countries, in charge of supervising the responsible and safe use of such technology. The United States Atomic Energy Commission was established in 1947 and the Committee of Supervision of Safe Conduct of Work in Industry and Mining Supervision was established in the Soviet Union in 1954.

Since then, the evaluation of the Nuclear Safety has been done by different Regulatory Commissions that work in permanent contact with the development and operation of Institutions working with Nuclear Technology. This feedback causes a constant improvement of the supervision making the Nuclear Technology one of the most reliable and safe industrial branches of the Society. 
Development and validation of a multi-scale and multi-physics methodology for the safety analysis of fast transients in Light Water Reactors

Furthermore, there are several international agencies as the International Atomic Energy Agency (IAEA), the World Nuclear Association (WNA) and the Nuclear Energy Agency (NEA) prioritizing the Safety Operation in the Nuclear Technology. Moreover, every country making use of this technology has an independent institution defining the regulation and standards that a NPP must meet for having authorized its operation. Examples of these institutions are the US Nuclear Regulatory Commission (USNRC), the Consejo de Seguridad Nuclear (CSN) in Spain or the Eidgenössisches Nuklearsicherheitsinspektorat (ENSI) in Switzerland, among others.

At this point and as it is well known, the operators of NPPs must obtain the corresponding license for operating each fuel cycle, among other actions such as obtaining the construct permit or evaluating the safety of the NPP in a specific situation. This license is obtained by reporting the results of the Safety Assessment to the Nuclear Authority and responding to any inquiry that this Authority may have about the status of the NPP. This Safety Assessment proves that the safety of the NPP is not compromised, assuring that the reactor core is cooled, that the radioactive material is confined and that the radiological protection requirements are met.

The law of the state is the maximum requirement that, as every member of a society, the NPP must meet. Nevertheless, the legislation of every country writes its requirements based on the ultimate effect of the malfunction of the NPP on the people and environment. The following paragraph is extracted from The US Code of Federal Regulations 40 Part 190, i.e. The Environmental Radiation Protection Standards for Nuclear Power Operations:

"This regulation limits the radiation releases and doses to the public from the normal operations of nuclear power plants and other uranium fuel cycle facilities - the facilities involved in the manufacture and use of uranium fuel for generating electrical power. The regulation sets limits on the annual dose equivalent to any member of the public to 25 millirem (0.25 millisievert ( $\mathrm{mSv})$ ) to the whole body, 75 millirem ( $0.75 \mathrm{mSv}$ ) to the thyroid and 25 millirem $(0.25 \mathrm{mSv})$ to any other organ. In addition, it specifies limits on the quantity of radioactive materials entering the general environment per gigawatt-year of electricity produced. These limits are 50,000 curies (1850 terabecquerels) of krypton85, 5 millicuries (185 megabecquerels) of iodine-129 and 0.5 millicuries (18.5 megabecquerels) combined of plutonium-239 and other alpha-emitting transuranic radionuclides with half-lives greater than one year".

The different States base their current legislation following the guidelines and recommendations of these International Organizations. In the end, the legal basis for the final limits of the exposure is defined by the legislation of the State. The legislation can vary from one State to another. The following paragraph is a translation of the Swiss Legislation about the regulation of the Radiological Protection in Nuclear Facilities SR 814.501: 


\section{"Chapter 2: Exposure of the population}

Art. 22 Dose limits for persons from the general public

1. The effective dose shall not exceed the limit of $1 \mathrm{mSv}$ per calendar year.

The organ equivalent dose shall not exceed the following limits:

a. for the eye lens: $15 \mathrm{mSv}$ per calendar year;

b. for the skin: $50 \mathrm{mSv}$ per calendar year."

In addition, it is common that the Legislation states which kind of events and causes leading to the release of radioactive material are to be accounted by the Nuclear Facility. The intention of regulating these facts is no other than assure the responsibility of the NPP in accounting all the types of accidents against the NPP must be prevented.

However, the philosophy of the Nuclear Industry is to avoid the scenarios where these measures of radiation would have to be evaluated. For this reason, the Nuclear Regulator, based on the Legislation of the State, sets a series of recommendations in order to make the NPP to account different scenarios that can potentially lead to the release of radioactive material.

These postulated scenarios are based on the international shared experience gather during the application of the Nuclear Technology since its beginning in the 50s. Moreover, these regulations are updated periodically based on the new experience and development of the Industry and Science. The postulated scenarios help to standardize the Safety Assessment in both national and international scope. These scenarios classify the events according to the challenging of the barriers of the power plants, and the possible initiating events. Nevertheless, the Nuclear Regulator can only postulate a list of generic scenarios and it is task of the NPP to fulfil and extend this list to the possible and specific events of the NPP and its design particularities.

The postulated scenarios evaluate the integrity of the barriers, setting limits according to physical parameters that are to be proven of not being trespassed. Assuring that, during the postulated scenario, the evolution of such parameters does not trespass the corresponding limit, the integrity of the barrier is granted. Assuring the integrity of the barrier, it is accepted that there will be no radioactive material released, and that the legal limit of dose will not be reached.

For that purpose, the licensee of the NPP uses simulation codes that reproduce the conditions of the scenario and predicts the evolution of the target safety variables. These codes are the state-of-the-art (SOA) of the simulation tools and are acknowledged by the Nuclear Authority as reliable and validated tools that have a coherent and representative prediction of the evolution of the proposed scenario. The Nuclear Authority acknowledge the capability of the simulation tools through a qualification process. When 
Development and validation of a multi-scale and multi-physics methodology for the safety analysis of fast transients in Light Water Reactors

the simulation tool meets the requirements of this qualification process, and the developers or users submit to the Nuclear Authority the corresponding documentation, the simulation tool becomes licensed. The reader must notice that this simulation tool licensing is usually done for the evaluation of specific phenomena. This means that each phenomenon to be analyzed regarding safety analysis may need different licensed simulation tools. In addition, the safety analysis does not need necessary to be restricted to the use of a single simulation tool or code. Different entities, like fuel vendors, develop extended methodologies that include the use of different codes for the analysis of the target phenomenon. These methodologies are required to be licensed as well, in the same procedure as the single codes, for the Nuclear Authority.

However, the analysis methodologies can evolve and improve. A new methodology for evaluating the Safety Assessment can present innovations in its procedure, new consideration for the physics of the core or any updating of the simulation models. In this case, the licensee will have to validate these new features in order to prove to the Nuclear Authority that the reliability of the method is consistent for safety analysis.

These safety requirements have been enhanced lately since the application of the Nuclear Technology has revealed the corresponding limitations in certain cases, for instance, the need of consider a six-equation two-phase flow model, instead of Homogenized Equilibrium Model of the fluid. For instance, the IAEA reported in 2016 this issue during the Convention on Nuclear (IAEA SAFETY STANDARDS SERIES, 2006). As a consequence, future operation strategies of NPPs as well as future design and construction must account the requirements regarding Safety Issues.

On the other hand, the economic system heads the operation of NPP to be competitive and this objective must not be a detriment to the safety. This inherent contradiction is managed by means of investing in formation, Research and Development (R\&D) capable of designing realistic and efficient simulation tools and methodologies. The aforementioned simulation tools simulate the analyzed scenario by discretizing the geometry of the target case, defining and accounting the physical models regarding such case and solving the time dependent problem with the corresponding numerical methods. Likewise, a simulation methodology would manage the data wisely to apply the corresponding simulation tool to the corresponding scale and accounting the corresponding physics. In other words, a methodology joints the capabilities of the SOA tools to provide a standard procedure of obtaining the safety evaluation of the target scenario.

On account of the need of assuring a safety operation of NPP in a cost-efficient way, it is necessary to design advanced methodologies that take advantage of the SOA tools predicting realistic values of the safety variables to be analyzed.

\section{WHY A METHODOLOGY?}

The evolution of the Nuclear Technology is the result of different efforts made by the R\&D Institutions and the NPP operators, and Owners Groups. The sharing of the knowhow is an important aspect, not common in other fields, that has favored the buildup of 
knowledge in the nuclear engineering field. Moreover, the evaluation criteria for the safety operation is also shared worldwide. The different Regulatory Authorities set similar requests that must be met in order to allow the operation of the corresponding NPP. The Nuclear Authority of each country has their own recommendations, but in general terms, the procedures for controlling the operation of NPP follow similar standards.

The reader must notice that the regulation and standards are in constant feedback with the developers and designers of the technology used in the NPP. A very reliable source of the technical specifications and limitations of a NPP is the designer and manufacturer of the reactor core and related systems and components. The company General ElectricHitachi (GE-H) holds the greatest sector of the market, and therefore, there is a strong relationship in the knowledge of the technical aspects of the LWRs developed by GE-H and the Nuclear Regulators.

The sharing of the technical specifications of the LWR is used to yield the postulated events against which the NPP using this type of reactor cores must be prevented. Therefore, it is common to observe that Nuclear Regulators as the Consejo de Seguridad Nuclear (CSN) of Spain, define the requirements based on the acknowledge specifications of the Core Designer.

This fact has derived in the use of standard procedures that the operators of the NPPs have to follow and present to the corresponding Nuclear Authority. The present thesis work focuses on the development of a simulation methodology for the analysis of those parameters that are relevant for evaluating the acceptance criteria set by the Nuclear Regulator, for the different postulated scenarios. By means of simulation tools and methodologies, the NPP or specific systems or components are modelled in order to predict their behavior during the scenario to be analyzed.

This fact leads to standardize the methodologies used for the evaluation of the different transient types to be simulated. For example, transient cases defined as Loss of Coolant Accident (LOCA), are accidents considered to occur with a certain probability and causing a certain degree of damage. It can be said that the methodology to simulate the consequences of a LOCA event has a standard procedure that several NPPs adapt to their specific case (Martin \& O’Dell, 2005).

On the one hand, it can be concluded that a methodology is necessary in order to provide the Nuclear Authority with the necessary data that assures the safety operation of the NPP. First, the methodology must be defined, selecting the most suitable simulation tools according to the needed physics and the degree of detail needed for the scale of the problem. Once the methodology has been validated, the NPP will count on a standard procedure to perform the safety analysis that the Nuclear Authority will ask for every Fuel Cycle.

On the other hand, a multi-scale and multi-physic simulation methodology is a useful tool for realizing scope analysis. The fuel cycle is designed in order to optimize the resources of the NPP for developing the power that is going to be sold. A multi-scale and 
Development and validation of a multi-scale and multi-physics methodology for the safety analysis of fast transients in Light Water Reactors

multi-physics methodology can be used for supporting and give a second opinion regarding the nuclear safety analysis. In addition, the Best Estimate (BE) approach will always tend to reduce the safety margin by means of predicting more realistic values. Therefore, the use of a BE multi-scale multi-physics methodology is a step ahead in the simulation of the NPP behavior for operation and safety analysis. In addition, every NPP has Nuclear Units that have been adapted in a unique way to the functions and purpose of the operator, owners and regulators. Therefore, adapting a methodology to the specific characteristics and features of the NPP results in a competitive tool for the safety production of electricity.

\section{WHY A BEST ESTIMATE?}

The operation of NPPs has experienced certain qualitative and quantitative steps since the beginning of the use of Nuclear Technology with civil purposes. A significant milestone is the extension of life of NPPs (Pavlovski \& Ilyn, 1987), (IAEA NUCLEAR ENERGY SERIES, 2015). Moreover, in 1974 the Title 10 part 50 of the Code of Federal Regulations (10 CFR 50) was formulated, and 10 years later, the 10 CFR 50.46 (USNRC, 2017 c) introduced the allowance to use BE codes. This decision was elucidated once the operative nuclear facilities where coming to the end of the operation license. Back then, the initial design of NPPs revealed that the assumptions taken where conservative (IAEA Safety Report Series, 2008), (IAEA NUCLEAR ENERGY SERIES, 2009). Therefore, after the analysis based on the operation experience, the corresponding authorities and experts in the field of Nuclear Technology concluded that there was still margin to extend safely the operation of NPPs until further license revision.

In addition to the overcoming of the conservative analysis in lifetime of Nuclear Facilities, another criterion was reviewed. Once again, the experience with NPPs showed that higher power rates were able to be obtained without trespassing the safety boundaries.

Moreover, the evaluation of the initial conservative criteria regarding design and operating conditions of NPPs, one must consider the development of the tools used for the analysis of the behavior of the Nuclear Core and the rest of the Power Plant. As it is known, the limits of the operation conditions in Nuclear Facilities are usually evaluated by means of computational simulations. This makes computer technology a cornerstone of the Nuclear Safety Analysis. This branch of technology develops at great velocity and hence, the capability of simulating the physics of the NPPs has evolved in relative short term. The improvement of the computational tools leads to more realistic evaluation of the safety analysis and therefore, to new re-evaluations of the safety margins.

At this point, the reader should have realized that a recurrent topic is implicit on the NPPs life: the evaluation of safety margins. The appliance of R\&D in the nuclear technology is key factor in the solution of the contradiction of needing a reliable and safety energy source versus the economic efficiency requested by the market. One must accept that NPPs are surveilled by Institutions, the so-called Nuclear Regulators that assure the safety operation by means of thorough safety margins, and also keep an update of those 
requirements according to the experience and the development of technology. Consequently, the way to achieve the most competitive operation conditions is to perform the most accurate prediction possible of those limits.

All the exposed points lead to the need of BE capability for safety analysis with simulation tools. Conversely to the Conservative evaluation, the BE approach takes advantage of the newest computational technology and experimental data to define more detailed models and to account more complex physical phenomena and the interaction between the different physics driving the behavior of NPPs. Further sections will explain in more detail the comparison between both approaches, but in principle, it is understandable that the BE approach can be useful in two aspects. On the one hand, it adjusts the operation of the NPPs by reducing the margin in operation and design, and heading to more competitive solutions. On the other hand, it allows a better understanding of the different phenomena occurring in the NPPs. The BE approach allows the analysis of detailed and local physical behavior that can be tackled with safety purposes. A more conservative approach would instead analyze the whole behavior in a coarser model, covering the detailed phenomena with greater margins for boundary conditions and being unable to analyze them. Nonetheless, special attention is needed for detailing the aspects of what is considered a realistic calculation and which further considerations are to be assumed in such type of calculations. The further assumptions required for realistic and conservative calculations are also specified by the Nuclear Regulator, and will be commented in the following sections.

In this context, KernKraftwerk Leibstadt AG (KKL) signed and renewed different contracts with the Senubio (ISIRYM/UPV) research group for the Development of a methodology for the analysis of fast transients for its Nuclear Reactor using the SOA tools. As explained above, KKL must periodically meet the requirements stated by the Swiss Nuclear Regulator (ENSI) and also is interested in having technical support for its own engineering work. Since KKL counts on different SOA tools for its different needs, the analysis of its status and future strategy yielded the motivation for adding value to its current capacities by the developing a methodology for safety analysis suitable for its objectives. The framework of this project has been the economic motivation for developing this $\mathrm{PhD}$ thesis work.

To sum up, the motivation of the work presented in this document comes from two circumstances. One of them is the analysis of the current technological and scientific status of the simulation tools and methodologies for the Safety Analysis of Nuclear Power Plants, that has been done by the author and supervisors of this thesis work. The other one is the parallel analysis and conclusion of the engineering staff of KKL and the resources invested in this common objective. 
Development and validation of a multi-scale and multi-physics methodology for the safety analysis of fast transients in Light Water Reactors

\subsection{Objectives.}

The objective of the thesis work is to develop a methodology for the analysis of transient scenarios of Light Water Reactors. This methodology works modular-wise using the available SOA codes at each step for working at a specific size scale, and applying the corresponding physics to the problem. The application of the methodology can be used modular-wise or simulating hierarchically step-by-step.

Milestones of the development of the thesis work:

- Modelling the steps of the methodology: This step is done to set the codes needed to be used and in which order. It must define how the flow data is going to act. And most important which safety variable needs to be predicted according to the corresponding Regulatory Guidelines.

- Generation of Cross Section Libraries: This step sets a procedure to generate the corresponding Cross Section Libraries to be used by the 3D NK code.

- $\quad$ System level simulation of the coupled NK and TH: For this step, an overall prediction of the system is obtained.

- Core level simulation of the coupled NK and TH: This step includes a channelby-channel modelling so the critical fuel channel can be tracked.

- Fuel level simulation of the TH: The simulation of this step uses the boundary and initial conditions of the critical fuel channel loaded in a more detailed pinby-pin model. This step will allow locating the critical fuel pin.

- Pin level simulation of the thermo-mechanics: This step is designed to evaluate the fuel behavior that lacks from the previous step. In addition, it will locate the critical axial node of the fuel pin.

- Uncertainty and sensitivity analysis: In order to complement the BE prediction of the safety variable a U\&S Analysis is proposed for the target output variable set as objective of the application of the methodology. The aim is to define the uncertainty margins of the analyzed variable and provide information of which input variables are more relevant for such uncertainty.

\subsection{Organization of the contents of the thesis}

The first chapter has presented the objectives and motivation of this thesis work. Chapter 2 will describe the state-of-the-art of the Safety Analysis in the field of the Nuclear Power Plants, more specifically, in the ones using Light Water Reactors. The state-of-the-art is 
also extended to the different physics analyzed for this purpose and the simulation tools used. Chapter number 3 describes the methodology as a procedure for the safety analysis of fast transients and its features. Chapter 4 is used to include parallel results developed within this thesis work, that are highly related to the presented main topic. The parallel work of this thesis yielded results in the validation and verification of different parts of the methodology, and two application cases are presented. Chapter 5 shows an application case of the methodology, specifically for the Turbine Trip Event of fuel cycle 18 in KKL. The conclusions and ongoing work is described in chapter 6 . And finally, Chapter 7 lists the contributions presented by means of journal papers, congress attendance, research projects and so forth. 



\section{Chapter 2 State-of-the-art}

This subsection details the main topics regarding the basis of the thesis work. The topics are, first of all, the definition of the concepts included in the Safety Analysis and the subject of the use of the methodology which are the postulated transient cases. Secondly, the current status of the simulation analysis techniques which are the Best Estimate Plus Uncertainty (BEPU) or Conservative approaches (D'Auria et al., 2006). Thirdly, the main features of the methodology developed in the thesis work which is the multi-scale and multi-physics concepts. And lastly, the explanation of the SOA simulation codes that are used in the methodology and currently, in this branch of the nuclear engineering.

\subsection{Safety Analysis.}

The basis of the Nuclear Safety is to provide physical barriers between the radioactive material and the workers from the NPP, the public and the environment. The efficiency of the barriers must be assured for normal and abnormal operation, whether anticipated or postulated incidents.

For Light Water Reactors (LWRs) the physical barriers consist of the fuel rod, made of the fuel pellet itself and the fuel cladding, the coolant pressure boundary, the primary containment and the reactor building, as Figure 2.1 shows. 


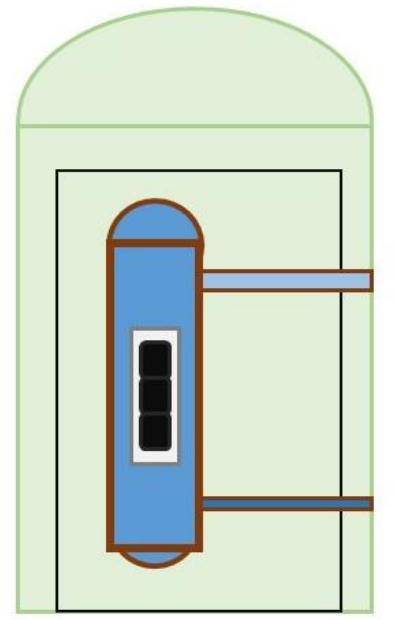

Figure 2.1 Scheme of the physical barriers containing the radioactive material.
FUEL PELLET

FUEL CLADDING

COOLANT PRESSURE BOUNDARY

PRIMARY CONTAINMENT

REACTOR BUILDING

In terms of the Deterministic Safety Analysis of NPP Guideline of the IAEA:

"A safety analysis of the plant design shall be conducted in which methods of both deterministic and probabilistic analysis shall be applied. On the basis of this analysis, the design basis for items important to safety shall be established and confirmed. It shall also be demonstrated that the plant as designed is capable of meeting any prescribed limits for radioactive releases and acceptable limits for potential radiation doses for each category of plant states, and that defense in depth has been effected."

The safety strategy of a NPP is based on three points:

- Preserve the integrity of the barriers.

- Prevent the occurrence of event.

- Limiting the consequences of a possible accident.

Regarding to the explained points, the principle of the defense in depth can be summarized as follows:

The studied measures that are intended to prevent the occurrence of errors do not avoid the assumption that accidents are always possible to happen. Hence, provisions have to be taken for dealing with them with the aim of restricting the consequences in the acceptable levels. In addition, Safety Analysis accounts a higher level of concern by considering effects having unexpected initiating events but also that may cause potential damage to the NPP. 
The willing of the international community in evaluating the different risk scenarios and assuring the safe operation of NPP is materialized in organizations like the International Nuclear Safety Group (INSAG). This organization gathers international experts of the nuclear field with proven competence and experience in Safety Issues. The working field of these experts are Nuclear Regulator Organizations and Research and Academic Institutions, as well as the industry. The aim of this group, hosted by the IAEA, is to work as an Authority in the field for giving advice and providing recommendations regarding the actual status of NPP and the ones being in current development.

There are five levels of defense in depth regarding the documentation of the International Nuclear Safety Advisory Group (International Nuclear Safety Advisory Group INSAG10, 1996):

Prevention of abnormal operation and failures: The preliminary design of the installations in a NPP must be an exhaustive study of its predicted operating conditions. This study must determine the worst cases for stresses in the different structures, regarding mechanical, thermal and pressure strain. A set of clearly defined and qualified rules have to be used to design, construct, check, install, test and operate the components of the different structures in the nuclear facility. The rules will allow adequate margins regarding the specific limits assuring the safety of the installation. The system dealing with abnormal situations using these margins will operate only in the corresponding situations, and will not actuate on an everyday basis.

This level includes the design of man-machine interfaces and other measures to diminish the operation staff stress hazards. Furthermore, the site selection is a relevant part of this level of defense. This selection is made regarding seismic levels, meteorological conditions, etc., as well as the awareness from external hazard sources that can affect the normal operation of the NPP.

Control of abnormal operation and detection of failures: The facility must count on reliable regulation, control and protection systems able to inhibit undesirable performance of the equipment before it is loaded beyond its rated operation conditions. This includes control systems for temperature, pressure, nuclear power and thermal power. The objective is to have a plant design with a stable core and high thermal inertia for it is feasible to hold the NPP within the authorized limits this way.

The measures for the purification systems and barriers will be checked as well as the radioactivity levels of certain fluids and atmosphere. Regarding the protection system, mainly the emergency shutdown system, will be able to solve any undesirable event. All these measures will be supported by a periodic equipment of surveillance, in order to spot any incident or abnormal situation.

Control of accidents within the design basis: Despite the efforts put on the first two levels, it is necessary for the sake of safety, to postulate a set of incidents and accidents in the NPP. Taking into account these events assures the reliability of the facility in more situations than the normal operation. The measures accounting accidents in the design 
Development and validation of a multi-scale and multi-physics methodology for the safety analysis of fast transients in Light Water Reactors

basis will limit the effects of them to acceptable levels, even if this means the disabling of design and installation safety systems for normal operating conditions. In these procedures, the human intervention will be limited to the corresponding time lapse allowing a carefully considered diagnosis to be reached. The main objective will be ensuring the core structure integrity by means of cooling procedures. The releases to the environment will be consequently limited.

The evaluation of which events are considered for the groups of accidents within the design basis will be carefully studied in the beginning of the design phase of the project. This is made in order to integrate the measures for this kind of accidents in the overall installation design.

Control of severe plant conditions including prevention of accident progression and mitigation of severe accident consequences: This level accounts the response in case of multiple failure. The target is the plant situations bypassing the first three levels of defense in depth. These situations are able to lead to core meltdown and hence, to higher radioactive release levels. Consequently, the concern of this level is to reduce the probability of such situations preparing the corresponding requirements and equipment to withstand this kind of scenarios.

The measures include the limitation of radioactive release in the frame of a very serious event that would had not involved core meltdown. Measures also have to gain time to set the protective features for the public in the vicinity of the site. For this reason, the containment function must be maintained under the best conditions. Further accident management will be defined in the emergency procedure.

Mitigation of radiological consequences of significant off-site releases of radioactive materials: The last level takes into account the release of radioactive material and the requirement of protecting the public. This case is presented when all of the previous levels have been trespassed. The basis of this level is the evacuation or confinement within the scope of the public authorities. It also includes the checking of foodstuff, distribution of stable iodine tablets, etc. All these measures will be included in the external emergency plans.

The conservatism of the safety margins is closely related to the deterministic approach developed in this thesis work. In fact, the work is focused on the first three levels being the ones where this approach applies. The different levels are intended to be independent, because of this, it is necessary to assure that the same event will not affect several levels simultaneously.

The role of the methodology developed in this thesis work is directly connected to the need of predicting if the safety of the operation of the LWR is being trespassed or not. The safety evaluation is determined by the dose received by the public, workers or the environment. However, it is possible to move this evaluation to the variables that define whether the barriers are trespassed or not. 
For example, the safety analysis is focused to the evaluation of the fuel cladding integrity. The fuel cladding is made of a material that changes its mechanical properties according to the temperature. Achieving certain temperature and regarding the external and internal stresses of the fuel cladding will affect the integrity and may cause its rupture breaking this safety barrier. In this case, the methodology of this work would use the physics implemented with numerical methods in the corresponding safety analysis code to predict the temperature evolution in a defined transient and hence, evaluate the consequences in the fuel cladding integrity.

The basis of the licensing of a NPP is to demonstrate according to the prediction of certain variables if the safety barriers are not trespassed for a list of transient scenarios. These proposed scenarios are based on the built up experience of the operation of NPP and the up-to-date knowledge of the Safety Analysis. The possible scenarios are analyzed systemically by the Reactor Designer and evaluated by the Nuclear Authority. These postulated transient cases are defined for each NPP internally based on the Design Basis Documentation and approved by the corresponding Nuclear Regulator. Moreover, the Nuclear Regulator can propose a minimum of events that are compulsory to be analyzed, as it is for the Swiss case (ENSI, 2019).

\subsection{Safety Analysis in a Nuclear Power Plant.}

The safety analysis of a Nuclear Power Plant intends to demonstrate three main points: First, that the principle of defense in depth is met. Secondly, that the corresponding safety barriers work properly in order to control the release of radioactive material. And, in third place, that the multiple protection levels against the failure of those barriers work efficiently. It must be account, that in order to satisfy these three points, neither the designers of the NPP nor the operators cannot use experimental procedures in order to elucidate consequences. Therefore, analytical procedures have to be accounted.

The evaluation of the risk in the operation of the NPP are considered accounting two aspects: Damage and frequency. Therefore, the experience in the analysis of the behavior of a NPP defines two methods: Transient Analysis (Deterministic method) (Pusa \& Isotalo, 2017) and Probabilistic Safety Assessment (remainder risk analysis) (Martorell Aigües, 2019).

The nuclear industry started to consider the Probabilistic Safety Assessment (PSA) after Three Mile Island (USA) accident (Wall, 1980).

As the IAEA defines in the Guideline for Probabilistic Safety Assessment (IAEA, 1992):

"Probabilistic safety analysis provides a comprehensive, structured approach to identifying accident scenarios and deriving numerical estimates of risks."

That event revealed that transient happening in a NPP could drive the operation beyond the design basis. This was caused due to an initiating event coming from not considered initial conditions. Therefore, PSA evaluates the frequency of core damage and quantifies 
Development and validation of a multi-scale and multi-physics methodology for the safety analysis of fast transients in Light Water Reactors

this probability accounting the possible initiating events and the sequence of events that may drive the NPP operation to multiple failure. The PSA engineers use a series of techniques based on the quantification of the inability of the protection systems due to maintenance, tests or failure. This data is structured in sequence trees in order to determine the possible evolution of incidents, their consequences and the probability and damage.

The analysis of remainder risk is complemented with the Deterministic Analysis. The also called Transient Analysis is the main topic in which this thesis work is developed. This method of analysis is based on the selection of certain transient events grouped according to their damage and frequency. These events are considered because they are assumed to challenge the protection barriers due to thorough conditions. Hence, the NPP is driven in these transient events to operation conditions where a previously imposed Acceptance Criteria must be meet. In other words, depending on the transient case, the integrity of the barriers can be evaluated regarding certain safety variables, such as maximum Vessel pressure.

According to IAEA definition from the Deterministic Safety Analysis (IAEA NUCLEAR ENERGY SERIES, 2009):

"The aim of the deterministic approach should be to address plant behavior under specific predetermined operational states and accident conditions and to apply a specific set of rules in judging design adequacy."

It must to be accounted that the derived variables are selected as a figure of merit representing the integrity of the barrier that is being compromised by the conditions of the transient event. For instance, a LOCA event may compromise the fuel cladding due to an excursion on the temperature, caused by a deterioration of the heat transfer capacity between fuel and coolant.

In this postulated event, the safety is guaranteed by assuring the integrity of the fuel cladding as a barrier against the release of fission products and radioactive material. The deterministic safety analysis translates this criterion in the analysis of certain physical parameters. According to the physical knowledge and scientific experience, it is known that the fuel integrity is held if fuel cladding does not lose its mechanical properties. Therefore, it is necessary to know which conditions derived from this kind of event make the cladding losing its mechanical properties. The Deterministic Safety Analysis leads then to the following facts:

1. The cladding keeps its mechanical properties if its molecular structure is not altered with oxidation and uptake of hydrogen.

2. The cladding keeps its mechanical properties if its temperature does not arise above certain value.

In a more detailed analysis, it could be added that both circumstances, oxidation and increase in fuel cladding temperature, are also related one each other. Consequently, and 
as an overview, Deterministic Safety Analysis has translated the evaluation of the safety limit for this accident, in the analysis of certain physical parameters that define a limiting value. The prediction of the evolution of these variables during the postulated scenario will proof that the Safety Limits are held. 
Development and validation of a multi-scale and multi-physics methodology for the safety analysis of fast transients in Light Water Reactors

\subsection{Transient events in Light Water Reactors.}

As prior sections explain, the Deterministic Safety Analysis is used to evaluate whether the operation of the NPP is safe by means of controlling that the safety limits are not trespassed in certain scenarios. These scenarios are hypothetical transient events that may occur in the lifespan of a NPP, and yield the plant to thorough conditions where the safety barrier must assure their effectivity. These transients consider a variation from the expected and nominal behavior of the NPP to another state in a time lapse. The size of this time lapse will categorize the transient in fast or slow. The point of this evaluation process is to consider that, if the NPP is able to operate safely in the worst-case scenario, it will be assured a complete safety operation during its life.

The reader must notice that the Nuclear Regulator only specifies which conditions and anomalies, as well as the causes leading to them, are to be accounted by the NPP. It is the task of the NPP operator to generate and define every possible initiating event leading to such scenarios. These series of initiating events are to be defined in a realistic manner, according to the indications of the Nuclear Regulator and the specifications of the components, systems and structures existing in the NPP.

As commented, a specific set of transient events are considered for the NPP. This set of transient cases can be classified regarding the severity of the consequences and the probability and frequency with which they can happen. Moreover, the categorization of the possible events to be evaluated can vary if the Nuclear Regulator considers so. Table 2.1 summarizes the classification of the transient events in a NPP.

Table 2.1 Type of operational states of a NPP.

\begin{tabular}{|c|c|c|c|c|c|}
\hline \multirow{2}{*}{\multicolumn{2}{|c|}{ Operational states }} & \multicolumn{4}{|c|}{ Accident conditions } \\
\hline & & \multicolumn{2}{|c|}{$\begin{array}{l}\text { Accidents within the de- } \\
\text { sign basis }\end{array}$} & \multicolumn{2}{|c|}{$\begin{array}{l}\text { Accidents beyond the de- } \\
\text { sign basis }\end{array}$} \\
\hline \multirow[t]{2}{*}{$\begin{array}{l}\text { Normal } \\
\text { operation }\end{array}$} & \multirow[t]{2}{*}{$\begin{array}{l}\text { Antici- } \\
\text { pated op- } \\
\text { erational } \\
\text { occur- } \\
\text { rences }\end{array}$} & \multirow[t]{2}{*}{$\begin{array}{l}\text { Accident } \\
\text { conditions } \\
\text { encom- } \\
\text { passed by } \\
\text { design basis } \\
\text { accidents }\end{array}$} & \multirow[t]{2}{*}{$\begin{array}{l}\text { Design basis } \\
\text { accidents }\end{array}$} & $\begin{array}{l}\text { Accidents be- } \\
\text { yond design } \\
\text { basis with no } \\
\text { significant } \\
\text { damage }\end{array}$ & $\begin{array}{l}\text { Severe acci- } \\
\text { dents }\end{array}$ \\
\hline & & & & \multicolumn{2}{|c|}{ Accident management } \\
\hline
\end{tabular}

Naturally, from the safety and economic point of view, there is no sense in proving physically the consistency of the safeguards, and due to this, the simulation methodology acquires a relevant role in the Safety Analysis. The aim of a simulation methodology is 
allowing the plant analysts to evaluate the evolution of the variables that define the integrity of the barriers acting at each transient scenario proposed by the Nuclear Authority.

Moreover, it is not feasible to evaluate every scenario derived from the malfunctioning or failure of every component, system or structure of the NPP. Therefore, there is a relevant engineering task in defining a list of transient events that cover the spectrum of worst scenarios accounting the specifications of the Nuclear Regulator and the knowledge of the functioning of the components, systems and structures of a NPP.

The transient events proposed to evaluate the consistency of the safeguards of a NPP are, in general terms, based on the guidelines of the USNRC (USNRC, 2007a). It must be accounted that the specific case of the methodology developed in this thesis work is focused in, according to Table 2.1 Anticipated Operational Occurrences and Accidents within the Design Basis. Therefore, the transient cases considered by the Nuclear Authority are defined as follows, according to the different categorizations that the USNRC propose, namely by frequency and type.

\subsection{Classification of the events in a Nuclear Power Plant with LWRs.}

The classification of the transients in NPPs help the analysts to group the hypothetical events that could endanger the plant in different categories. By group events, the safety analysis and the corresponding measures to tackle the consequences are simplified and can be more effectively analyzed. There are different ways to classify the so-called Initiating Events, and it is task of the corresponding Nuclear Authority to set a consistent way to categorize and standardize them. The most common ways are based either on the probability of occurrence of the event or on the change of the main core variable affected in the transient, e.g. core pressure or coolant temperature.

\subsubsection{Classification according to probability of the Initiating Event.}

For the first classification method, there are two options: Anticipated Operational Occurrences (AOO) and postulated accidents.

Anticipated Operational Occurrences: These are events that are expected to happen with a moderate frequency. AOOs can be also classified between those occurring several times during the plant operation. And those that may occur during the life of the plant. Table 2.2 (USNRC, 2007a) shows examples of possible AOOs for LWRs, and which of PWR or BWR facilities is affected by the corresponding transient event. USNRC names these events as Condition II and III events. 
Development and validation of a multi-scale and multi-physics methodology for the safety analysis of fast transients in Light Water Reactors

Table 2.2 Transient cases for BWRs and PWRs.

\begin{tabular}{|c|c|c|}
\hline EVENT & PWR & BWR \\
\hline Inadvertent control rod or rod group withdrawal & $\checkmark$ & $\sqrt{ }$ \\
\hline $\begin{array}{l}\text { Loss or interruption of core coolant flow, excluding re- } \\
\text { actor coolant pump locked rotor }\end{array}$ & $\checkmark$ & \\
\hline Inadvertent moderator cooldown & $\checkmark$ & $\sqrt{ }$ \\
\hline Inadvertent chemical shim dilution & $\checkmark$ & \\
\hline $\begin{array}{l}\text { Depressurization by spurious operation of an active el- } \\
\text { ement, such as a relief valve }\end{array}$ & $\checkmark$ & $\checkmark$ \\
\hline Loss of normal feedwater & $\checkmark$ & $\checkmark$ \\
\hline Loss of condenser cooling & $\checkmark$ & $\sqrt{ }$ \\
\hline Steam generator tube leaks & $\checkmark$ & \\
\hline $\begin{array}{l}\text { Reactor-turbine load mismatch, including loss of load } \\
\text { and turbine trip }\end{array}$ & $\checkmark$ & $\sqrt{ }$ \\
\hline Control rod drop (inadvertent addition of absorber) & $\checkmark$ & \\
\hline Single error of an operator & $\checkmark$ & $\checkmark$ \\
\hline Single failure of a control component & $\checkmark$ & $\checkmark$ \\
\hline Single failure in electrical system & $\sqrt{ }$ & $\sqrt{ }$ \\
\hline $\begin{array}{l}\text { Minor reactor coolant system (RCS) leak or loss of re- } \\
\text { actor coolant such as from a small ruptured pipe or } \\
\text { from a crack in a large pipe }\end{array}$ & $\checkmark$ & $\checkmark$ \\
\hline Minor secondary system break & $\checkmark$ & \\
\hline Loss of offsite power & $\checkmark$ & $\checkmark$ \\
\hline Operation with a fuel assembly in an improper position & $\checkmark$ & $\checkmark$ \\
\hline Inadvertent blowdown of RCS & & $\checkmark$ \\
\hline Loss of feedwater heating & $\checkmark$ & $\checkmark$ \\
\hline Trip of any or all recirculation pumps & & $\checkmark$ \\
\hline Inadvertent pump start in a hot recirculation loop & & $\sqrt{ }$ \\
\hline Condenser tube leak & & $\sqrt{ }$ \\
\hline
\end{tabular}


Table 2.3 (Continued) Transient cases for BWRs and PWRs.

\begin{tabular}{|l|c|c|}
\hline EVENT & PWR & BWR \\
\hline Startup of an idle recirculation pump in a cold loop & & $\checkmark$ \\
\hline Reactor overpressure with delayed scram & $\checkmark$ & $\checkmark$ \\
\hline
\end{tabular}

Postulated accidents: these accidents are unanticipated occurrences. This means that they are postulated, but not expected to occur during the life of the nuclear power plant. Table 2.4 (USNRC, 2007a) shows the postulated accidents for LWRs.

Table 2.4 Transient events in LWRs categorized as Postulated Accidents.

\begin{tabular}{|l|r|r|}
\hline EVENT & PWR & BWR \\
\hline $\begin{array}{l}\text { Major rupture of a pipe containing reactor coolant up to } \\
\text { and including double-ended rupture of the largest pipe } \\
\text { in the reactor coolant pressure boundary }\end{array}$ & $\checkmark$ & $\checkmark$ \\
\hline Ejection of a control rod assembly & $\checkmark$ & \\
\hline Control rod drop accident & $\checkmark$ & \\
\hline $\begin{array}{l}\text { Major secondary system pipe rupture up to and includ- } \\
\text { ing double-ended rupture }\end{array}$ & $\checkmark$ & $\checkmark$ \\
\hline Single reactor coolant pump locked rotor & $\checkmark$ & \\
\hline Seizure of one recirculation pump & & $\checkmark$ \\
\hline
\end{tabular}

\subsubsection{Classification according to variable change.}

As commented, there is another categorization criterion for the possible accident events happening in a NPP. This categorization is made according to the type of the event, i.e. its effect on the plant. This classification method is useful to categorize and organize analysis of AOOs and postulated accidents so that the analyst can compare them on common bases, effect and safety limits. These comparisons can help to identify limiting events and cases for detailed examination, omitting non-limiting cases for future considerations. For this reason, AOOs and postulated accidents are grouped in eight types:

- Decrease in core coolant temperature.

- Increase in core coolant temperature.

- Increase in reactor pressure.

- Decrease in reactor coolant flow rate. 
Development and validation of a multi-scale and multi-physics methodology for the safety analysis of fast transients in Light Water Reactors

- Increase in reactor coolant flow rate.

- Reactivity and power distribution anomalies.

- Increase in reactor coolant inventory.

- Decrease in reactor coolant inventory.

The consequences of this variation of parameters lead whether to the increase of the pressure of the nuclear system, or to the increase of the fuel temperature. These facts can result in the compromise of the integrity of the barriers containing and confining the radioactive material. An excursion in the fuel temperature can affect the integrity of the fuel pellet, but also the integrity of the fuel cladding by oxidation or other mechanic issues that derive in the embrittlement of the fuel rod. The increase in pressure can trespass the mechanical limits of the structures of the coolant pressure system or the reactor pressure vessel. Whatever of these barrier failures yield the release of radioactive material to another barrier level, and hence, must be accounted and evaluated. The following points explain the consequences in the variation of fuel temperature and system pressure of the postulated events.

\section{Decrease in Core Coolant Temperature}

Events that result directly in a core coolant temperature decrease are those that either increase the flow of cold water or reduce the temperature of the water being delivered to the reactor vessel. Core coolant (moderator) temperature reduction results in an increase in core reactivity, which increases the power level and threatens overheating of the fuel.

\section{Increase in Core Coolant Temperature}

Events that result directly in a core coolant temperature increase are those that increase the temperature of the water being delivered to the reactor vessel. Increases in core coolant temperature increase reactor pressure and threaten the reactor coolant pressure boundary. Generally, an increase in core coolant temperature results in a decrease in reactivity and a corresponding reduction in power. However, depending on the fuel design and temperature increment, an increase in core coolant temperature can result in a slight increase in reactivity and power.

\section{Increase in Reactor Pressure}

Events that result directly in significant reactor and nuclear system pressure increase generally are initiated by a sudden reduction in steam flow, generally initiated by the closure of the corresponding flow valve. Increasing pressure leads to an increase in coolant density and due to the neutronic feedback, core reactivity increases. This causes increasing of core power level, which may challenge SAFDLs. On the other hand, the mechanical limits of the RPV and the RCPB may be challenge as well. Reaching the setpoint of the relief valves may cause delivery of steam, that additionally must be analyzed regarding dose limits. 


\section{Decrease in Reactor Coolant Flow Rate}

Events that result in a reduction in recirculation flow rate decrease the reactor core coolant flow rate. Decreases in the reactor core coolant flow rate decrease the ability of the coolant to remove the heat generated in the core, which threatens overheating of the fuel. Decreases in core coolant flow also decrease coolant density and hence, decrease core reactivity, which decreases core power level and increases reactor water level.

\section{Increase in Reactor Coolant Flow Rate}

Events that result in an increase in recirculation flow rate increase the reactor core coolant flow rate. Increase in reactor core coolant flow rate result in an increase in coolant density and an increase in core reactivity. An increase in core reactivity increases core power level and threatens overheating of the fuel.

\section{Reactivity and Power Distribution Anomalies}

Events that result in localized positive reactivity insertions are generally caused by errors in the movement of control rods. Localized positive reactivity insertions cause anomalies in power distribution and an increase in core power level, which threaten overheating of the fuel.

\section{Increase in Reactor Coolant Inventory}

Events that lead to a feedwater flow increase greater than the steam production rate will result in an excess of coolant inventory and an increase in reactor vessel water level, which increases power due to the increase in core inlet sub cooling. Increasing the vessel water level can lead to a turbine trip which will cause a pressurization event characterized by a reactivity increase due to the coolant density increase. This increases core reactivity, thereby increasing reactor vessel and nuclear system pressure and core power level, which threatens over pressurization of the reactor coolant pressure boundary. 
Development and validation of a multi-scale and multi-physics methodology for the safety analysis of fast transients in Light Water Reactors

\section{Decrease in Reactor Coolant Inventory}

Events that lead to a steam flow rate greater than the feedwater input result in a decrease in the reactor coolant inventory. Decreases in reactor coolant inventory cause a decrease in reactor water level, which threatens overheating of the fuel, and a decrease in coolant temperature, which leads to a mild depressurization.

Regarding this classification, it can be analyzed which effect will have the considered event or which results will have that may challenge designated safety limits.

To summarize, each of the transient cases compromises, in a specific way, certain safety barriers of the systems in a NPP. The operator will have to evaluate according to the corresponding documentation which barriers are affected by each transient and how to apply the simulation methodology in order to grant that the performed simulation assures that the safety limits are not trespassed. At this point, it is necessary to define the features included in a simulation methodology and how they provide enough information to assure to the Nuclear Authority that the safety barriers are not compromised in the corresponding transient case.

\subsection{The use of simulation tools for the Safety Evaluation.}

A simulation methodology for the safety analysis is made of code calculations that use a model representative of the system or systems of NPP that play a relevant role in the transient case that is being evaluated. On the one hand, a simulation code is the result of programming the solution of the specific physics of the NPP behavior using numerical methods. These numerical methods are applied by means of discretizing the problem time-wise, and taking advantage of the spatial discretization defined in the model that is being simulated.

The Nuclear Authority does not specify which simulation methodology has to be used or which software is appropriate for each proposed transient case. The requirement is to assure that the methodology grants that the safety limits are not trespassed for the proposed events. For this purpose, the codes used in the methodology must be previously validated and verified.

The validation of a simulation code is done using the information provided by the different experiments that are available in the field of the Nuclear Technology (D'Auria \& Salah, 2006). In a validation process, the experiment is modelled for the code that is to be validated and the predicted results are compared to the experimental results. This procedure sets in which level the validated code is able to reproduce the physics of a transient. Moreover, the code must pass a series of validation procedures in order to assure that it represents the real behavior. These validation procedures compare the predicted results against analytic and/or real data coming from Experimental Test Facilities and NPPs. In addition, the code must be documented in detail for the users. 
Therefore, the operator uses the corresponding simulation code according to the requirements of the transient case proposed by the Nuclear Authority. The use of the simulation code will provide the operator with the evolution of the target variables that define the behavior of the integrity of the barriers. For example, a simulation code analyzing the thermo-mechanics of the fuel cladding, can be used to evaluate whether the deformation of the cladding overpasses or not the safety limits. Naturally, the target variables to be evaluated change depending on the transient case to be simulated. Consequently, the operator must define an appropriate methodology using the corresponding simulation codes and justifying the decisions.

However, the capability of predicting realistic results depends on several aspect of the simulation code and its design. It must be accounted that the Nuclear Authority does not request a specific level of accuracy predicting results rather than assure with a validated code, that there exists a sufficient safety margin between the allowable value for a target variable and the predicted value. In this aspect is where the different approaches of the Deterministic Safety Analysis can be relevant. Nevertheless, it applies first to define the evaluation of the safety margins.

The Safety Margins are designed according to the Acceptance Criteria proposed by the Regulatory Body of the corresponding state, derived from the Safety Analysis Standards of the IAEA. The basic Acceptance Criteria are the limits and conditions set by the Nuclear Authority which objective is assure the achieving of the corresponding safe operation conditions.

The description of the acceptance criteria may differ from the regulation guides of the different States. Moreover, can be described in both regulation and legal basis. However, the acceptance criteria can be summarized in the following points:

1. The doses received by workers, public and the individual must be within the defined limits and as low as possible for all the operation states. This is done by means of assuring the mitigation of the consequences derived the radiation released at any kind of accident.

2. The integrity of the barriers established by the defense in depth should be maintained in order to avoid the release of radioactive material. This must be done depending on the different categories for the accidents where the integrity is needed.

3. The cooling and heat removal of the fuel assemblies must be granted.

4. The reactivity of the core must be controlled.

5. The radioactive material must be confined.

The use of the acceptance criteria should be set for the complete range of normal operation and accident conditions. The acceptance criteria are related to the frequency of each possible event. Anticipated Operational Occurrences are events to happen with frequency and therefore, the acceptance criteria are more restrictive that those events, like Design Basis Accidents which frequency is lower. 
Development and validation of a multi-scale and multi-physics methodology for the safety analysis of fast transients in Light Water Reactors

For convenience, the acceptance criteria are set in terms of the variable or variables that analyze the behavior that can challenge the integrity of the corresponding variables. Nonetheless, it is common to use surrogate variables that set an acceptance criterion that assures that integrity of the barrier if the limit of this surrogate variable is not exceeded. The following are examples of such surrogate variables: Departure from Nucleate Boiling Ratio, built up of oxide in the fuel cladding, peak cladding temperature, and so forth. The following subsection explains the relevant surrogated variables. It is then, when the approach in the simulation analyses play a significant role. The uncertainty of the prediction of the corresponding variable must be quantified. Conservative approaches guarantee that the predicted value is sufficiently away from the safety limits of such variables. On the other hand, a BE approach means a more realistic prediction, therefore, the uncertainty of the prediction must be defined in order to evaluate if the upper or lower boundary of the uncertainty of the predicted value is within the safety limits.

\subsubsection{Surrogated variables used in the Deterministic Safety Analysis.}

At this point, is it necessary to detail the so-called surrogated variables for the Safety Analysis. The Deterministic Safety Analysis is the manner of providing quantitative proof of the evaluation of the safety. The requirement of not damaging a barrier preventing and containing the radioactive material is a generic concept that needs to be translated to specific terms. These specific terms find their evaluation by analyzing the physical behavior of the barriers during the postulated event, and calculating the evolution of physical parameters defining the integrity of the barrier.

For instance, let us consider the fuel cladding as barrier containing the Fission Gas Release from its inside. The cladding works as a structural barrier that avoids the spreading of the fission products whether in it is gaseous or solid form (as fuel pellet fragments). The cladding has been designed with materials and dimensions that optimize its functions as barrier, and other functions as transmitting the fission heat from the pellet to the coolant. The NPP must be designed to maintain the integrity of this barrier in a series of adverse postulated scenarios, in order to not releasing radioactive material that could suppose trespassing the dose limits to workers, public and environment. To accomplishing this legal requirement, it is necessary to stablish a deterministic value that defines in which conditions the integrity of the barrier is kept. Therefore, the Deterministic Safety Analysis defines the physical behavior of the core or NPP during a postulated event that could challenge the fuel cladding integrity as a barrier and defines the physical parameters defining his integrity. The integrity of the fuel cladding is challenged when it loses the mechanical properties of its design that makes it work as a barrier. Hence, evaluating the temperature of the cladding during the postulated event is a manner to determine the integrity of the barrier. It is a task of the engineering staff to define which barriers are challenged according to the postulated event, and which are the physical parameters that can define the integrity of these barriers. The Nuclear Regulator postulates a series of initiating events that could challenge the integrity of the barriers according to a variation of parameters. These postulated initiating events (see 1.2.1.4) have the ultimate effect in 
the temperature of the fuel cladding or the pressure of the primary cooling cycle. Nevertheless, there are other parameters that can also affect to the integrity of the barriers. The following list details a series of parameters that are commonly evaluated in the Deterministic Safety Analysis.

a. Peak Cladding Temperature.

The temperature of the cladding is an indicator of the integrity of this barrier. A peak value shows the higher value of a fuel rod or group of rods. Therefore, a value beyond the limit of $2200 \mathrm{~F}$ set for the Peak Cladding Temperature (PCT) means that this barrier is being compromised at some point. The PCT is set experimentally as a limit value for maintaining the mechanical properties of the fuel cladding (NEA, 2009). Higher values than the allowed cause modification in the microstructure of the specific type of alloy used for the fuel cladding. Zirconium-based alloys like Zircalloy-2, Zircalloy-4 and so forth showed, according to scientific and industrial experience, a phase change in its microstructure and chemical reactions affecting this microstructure for values of approximately $800^{\circ} \mathrm{C}$. The chemical reactions are caused by the interaction with the coolant in the operational conditions of a LWR. These reactions include oxidation and generation of hydrogen. The oxidation contributes to the generation of an oxide layer and the corrosion of the fuel cladding. The generation of hydrogen, on the other hand, can cause the absorption and generation of intergranular and intragranular hydrides affecting as well to the fuel cladding microstructure. Figure 2.2 shows the scheme of the different effects of the increase in fuel cladding temperature compromising the integrity of this barrier. 
Development and validation of a multi-scale and multi-physics methodology for the safety analysis of fast transients in Light Water Reactors

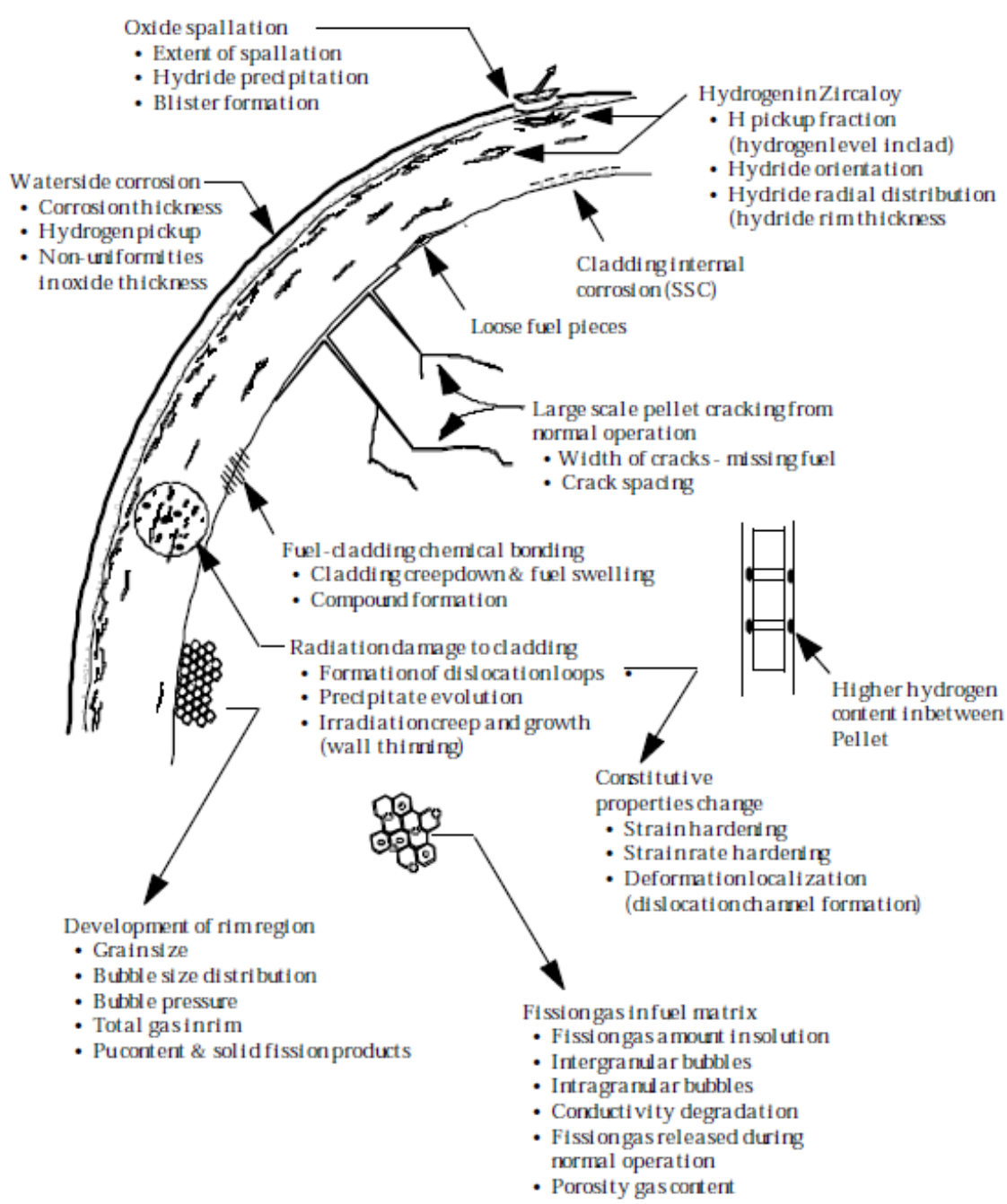

Figure 2.2 Phenomenology of the fuel cladding temperature increase beyond the PCT (NEA, 2009).

These phenomena are responsible of the embrittlement of the fuel cladding temperature. The embrittlement of the cladding makes it to lose the mechanical properties that allow the cladding to endure the conditions of the operation of a LWR. This barrier has the objective of enduring the pressure difference between the coolant pressure boundary and the pressurized internals of the fuel rod. An embrittlement of this barrier can derive in the burst of the cladding due to the pressure difference or the puncture due to excessive oxidation. The integrity of this barrier is held by assuring that the PCT is below $1480 \mathrm{~K}$ $\left(2200^{\circ} \mathrm{F}\right)$, according to the Regulatory Guidelines of the USNRC (USNRC, 2017). This 
safety variable is used as figure of merit in transient cases where the heat transfer capacity can be significantly affected, as in LOCA conditions. Nevertheless, depending on the results of the analysis of a LOCA, additional variables may be used, such as the oxidation rate of the cladding, coolability of the fuel assemblies and so forth. Regarding events of Condition II, the analysis of the effectivity of the heat removal capacity of the fuel rod by the coolant can be measured with the so-called Departure of Nucleate Boiling Ratio (DNBr) or the Minimum Critical Power Ratio (MCPR).

b. Departure of Nucleate Boiling and Critical Power Ratio.

The DNB makes reference to a change in the flow and heat transfer regime. The temperature of the fuel cladding, and hence its integrity, depends on the capacity of the coolant flowing through the fuel rods to remove the heat that is being generated in the fuel pellets. This capacity will be enhanced or deteriorated according to the changes in the two-phase flow of the coolant. There are several variables used to model the phenomena of the heat and flow regime in a LWR. These variables come from the accumulated experience and the research effort done by several institutions. This effort yielded different correlations that model the behavior of these flow and heat regime changes, most of them adapted or limited to specific conditions.

The importance to model these changes in the flow and heat transfer regime lays on the relevancy of defining which conditions drive to DNB or CPR limits. These limiting values can define where and in which conditions the phenomena of the dryout can take place. The dryout is the local removal of any two-phase flow layer in contact with the fuel cladding surface leaving the heat extracting task to the void phase of the flow. This means a sudden deterioration of the heat transfer capacity and consequently a sudden increase in the fuel cladding temperature. The local characteristics of this effect increase the difficulty of predicting these phenomena which can yield in severe fuel damage.

Therefore, transient scenarios driving to a deterioration of the heat transfer capacity are evaluated according to these variables, DNB and CPR, and variables derived from these ones. The application of these variables usually depend on the type of LWR. For instance, PWR do not experience two-phase flow regime in normal operation conditions as BWR do. For this reason, the change or departure of boiling heat transfer regime can be accounted as an anomalous behavior which, in BWR makes no sense since the departure of boiling is expected at certain height of the fuel rod in normal operation. Consequently, in BWR the deterioration of the heat transfer capacity is measure and analyzed with the CPR. The usual form of obtaining these parameters is setting a ratio between the nominal value of current heat generation in the fuel rod, in normal operation conditions, and the heat generation value in which the heat transfer regime would change. In this form, it is possible to evaluate how close is the current heat generation to a critical generation of heat that could incur in fuel damage. The evolution of this limiting heat generation of the fuel rod is commonly represented depending on the difference between the cladding surface temperature and the saturated temperature of the coolant. Figure 2.3 
Development and validation of a multi-scale and multi-physics methodology for the safety analysis of fast transients in Light Water Reactors

shows this function, representing the heat generation as surface heat flux of the fuel cladding.

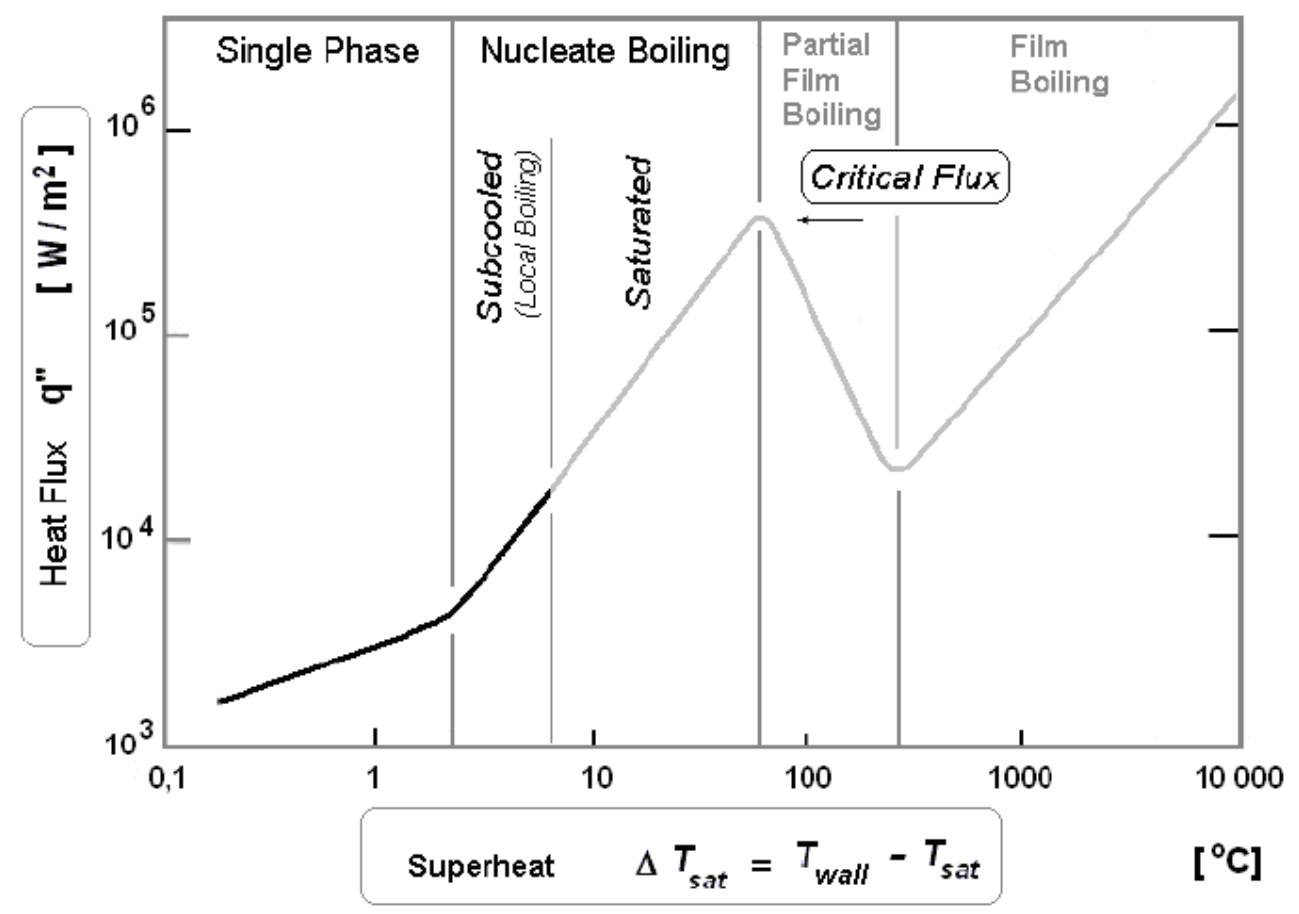

Figure 2.3 Behavior of the heat flux as a function of temperature difference between cladding surface and saturated coolant (NEA, 2009).

As Figure 2.3 shows, there is a critical minimum heat flux value through the fuel cladding that can cause fuel damage due to temperature increase. The different safety values based on the ratio between this limiting value and the current operational value are used for setting acceptance criteria when a transient scenario can drive to fuel damage due to deterioration of the heat transfer capacity.

c. Pellet Cladding Interaction.

An interesting phenomenon in the consideration of the integrity of the fuel cladding is the so called Pellet-Cladding Interaction (PCI). This effect results in an excessive stress in the fuel cladding caused by the contact in the inner side with the fuel pellet. The fuel pellet loses its original manufactured form due to the irradiation caused by the operation during the fuel cycle. This form loss is caused by different dilatation processes that fracture the pellet and increase its volume, pushing the pellet surface against the inner side of the fuel cladding. The local contact points can yield an excessive strain force that can become in fuel rod puncture or deformation. Figure 2.4 depicts the evolution of the PCI in three generic steps depending on irradiation (operation). This phenomenon is directly 
related to the gap closure, i.e. the definitive contact of the pellet and cladding inner surface removing any initial manufactured and designed gap.
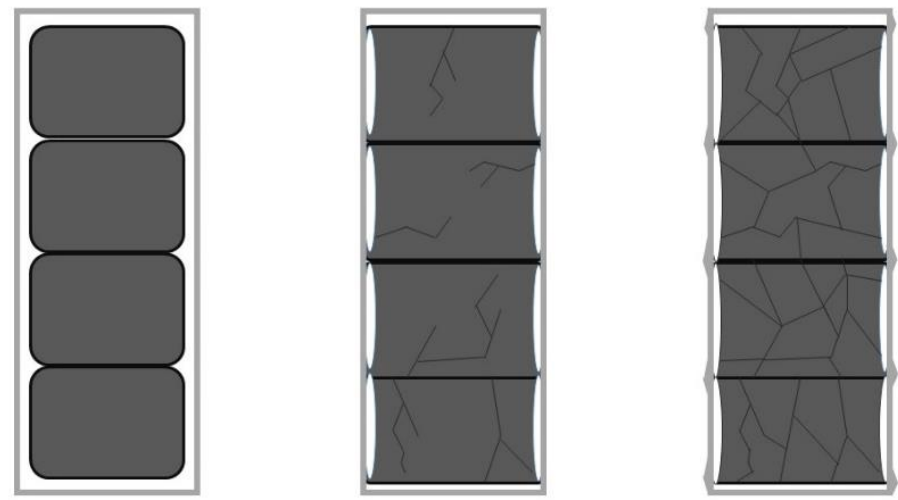

Figure 2.4 Simplified scheme of pellet-cladding system prior operation (left), irradiated and prior to PCI (middle) irradiated and after PCI (right).

The phenomena initiating the failure mode of the PCI spans from Stress Corrosion and Cracking (SCC), Embrittlement due to Hydrogen, delayed hydride cracking and so on (Michel et al., 2008). It is particularly difficult to elucidate which of these effects can cause the definitive failure of the fuel rod or if it is caused as a combination of all of them. The most relevant factors influencing the PCI evolution can be listed as follows:

- Design and fabrication process of the fuel rod: The density of the fuel pellet and the gas-gap size between pellet and cladding surface determines the time and behavior for achieving the so-called gap closure. Moreover, fabrication defects will contribute to the concentration of stresses in such points of the fuel pellet or cladding.

- Fabrication material for the fuel cladding: The criteria of the material selection must include the consequences of high temperatures and irradiation, especially for the inner side of the cladding. The design decision will affect the endurance of shear stresses in case of PCI.

- Operation conditions: Since the beginning of the analysis of these phenomena, special attention has been given to the operation conditions. Planning an operation without stiff power ramps can reduce the fuel pellet swelling and hence, the gas gap closure and final PCI effect. Moreover, the irradiation rate can be modified and accommodated with relaxation of axial power peaks. 
Development and validation of a multi-scale and multi-physics methodology for the safety analysis of fast transients in Light Water Reactors

The physics predicting the behavior of this phenomenon that can damage the barrier of fuel cladding are depending on the material properties and their reaction to chemical and mechanical effects. This fact requires the use of specialized simulation tools for a better understanding and prediction in the transient scenarios where these phenomena applies. The analysis of this and other fuel behavior safety variables introduce the need of thermo-mechanical codes in multi-physics safety analysis methodologies.

\section{d. Fuel Enthalpy.}

One of the common accident scenarios to account in nuclear safety analysis is the reactivity insertion accident (RIA), that can be caused among other facts by the sudden control rod removal during normal operation. The damage range of this transient case affect the integrity of the fuel rod, and the representative value of the evolution of this scenario is the fuel enthalpy or the deposited energy on the fuel rod due to this sudden increase in the fission rate. It can be understood that there exists a limiting increase rate of this variable that challenges the mechanical and heat transfer properties of the fuel pellet. The nuclear technology field has invested the corresponding effort analyzing these phenomena, supported by the accumulated operational experience and the research institutions with their test facilities. The results have yielded a series of specialized simulation codes focused on the modelling of the increase of fuel enthalpy and its consequences.

The dedicated experiments headed to elucidate the fuel behavior during a RIA are based on the analysis of the fuel enthalpy as a function of the fuel temperature and as a direct measure of the damage of this barrier (Rudling et al., 2016). The basic model from which the corresponding fuel behavior codes base their calculation schemes is described in Equation 2.1 for the $h_{f}$ as the enthalpy, $T_{f}$ as the fuel pellet temperature and $c_{f}$ as the heat capacity.

$$
h_{f}\left(T_{f}\right)=\int_{T_{0}}^{T_{f}} c_{f}(T) d T
$$

The measurement of the enthalpy can be set as a function of local variables such as burnup and oxidation (Beard et al., 2006). The local aspect of these phenomena is accounted by the use of BE simulation tools allowing the analysis of the event in such a reduced scale. It is hence a contribution for the enhancement of Safety Analysis the multi-scale feature and it is nowadays a requirement in analysis methodologies in nuclear safety. Moreover, the analysis of this phenomenon must include the dynamics of the reactivity insertion due to the control rod removal, whether control rod drop accident or control rod ejection. This fact must be added to the axial and radial power peaking factors, and therefore, the multi-physics feature of a safety analysis methodology plays also an important role for a $\mathrm{BE}$ simulation. 


\section{e. Cladding Deformation.}

For certain types of considered accident scenarios there is the requirement to assure the long term coolability. The coolability of the fuel rods depends on the ability of the nuclear system to allow the necessary coolant flow in contact with the fuel surface in a manner that can remove the generated heat or at least contribute to the convective heat transfer. For these conditions, it is necessary that the cross section area of the coolant flow through the fuel rod array has the corresponding designed size and shape.

According to this, there are different transient accident scenarios that can cause the deformation of the fuel rod array. These scenarios include the possibility of plastic deformation of the cladding due to mechanical forces or due to clad ballooning due to the loss of mechanical properties and high temperatures. Another factor to account is the bowing of the fuel rods, that could also prevent the flow path of the coolant.

For these cases, it is necessary to use fuel behavior simulation tools that consider not only the hoop and axial strains on the fuel cladding, but also the local feature of these phenomena.

\section{f. System pressure.}

The heat generation in LWR is transported by the coolant by means of achieving high values of enthalpy, i.e. pressure and temperature. This form of energy is run in a Rankine Cycle that has to be confined and maintained in the corresponding system or systems. These systems must endure certain stresses and fatigue without losing the integrity, since the primary cycle of a LWR operates as a safety barrier against the release of fission products.

The limits of this barrier are coded in the Nuclear Regulation (USNRC, 2019) and usually are analyzed as the pressure of the coolant calculated in the Reactor Pressure Vessel. This is the common point for calculating this pressure value during a safety analysis since it is the expected location of the higher value. The evaluation of the mechanical limits related to pressurized vessels, such as the RPV of NPP is based on the ASME regulation (Rao, 2009).

\section{g. Core Tie Plate Lift Forces.}

The flow path of the coolant in a LWR goes through the fuel assemblies developing a non-trivial drag force. The design of the reactor core must account the corresponding mechanical analysis to provide the upper tie plate with sufficient downforce. The reason is that the drag force of the coolant flow can cause the lift of fuel assemblies and consequent damage to the structure due to the sudden loads and impacts. Moreover, accounting the increase of the inlet coolant flow as a possible event for accident analysis, the resulting lift force applied to the fuel assemblies is used as safety variable. 
Development and validation of a multi-scale and multi-physics methodology for the safety analysis of fast transients in Light Water Reactors

The drag force responsible of the fuel assembly lift effect is described in Equation 2.2. Where $C_{D}$ is the drag coefficient, $A$ is the reference area, $\rho$ is the density of the coolant flow and $v$ is the velocity of the coolant flow.

$$
F_{D}=C_{D} A \frac{\rho v^{2}}{2}
$$

According to Equation 2.2, a thermal-hydraulic analysis is needed for modelling the resulting effects. The friction coefficient as well as the flow velocity are depending on the flow regime, whether laminar or turbulent. In addition, the flow density would depend in the thermal properties of the coolant. At this point, a BE analysis would add the consideration of the two-phase flow, providing a more realistic calculation.

The summarized series of safety variables of the previous list show how relevant for the $\mathrm{BE}$ simulation is to account not only the relevant physics of the analyzed phenomena but also the corresponding scale. Furthermore, there are additional derived variables that can be used for the assessment of the safety limits. In addition, these variables will have to be updated as long as the nuclear systems evolve in new designs that force to account new kind of transient phenomena that may compromise the integrity of the safety variables.

\subsubsection{BEPU methodologies.}

As the present document describes in the motivation section, the safety margin is an aspect of the nuclear technology for the energy production that plays an interesting role for an efficient and safe operation. The aim of a NPP should be achieving a competitive operation without compromising the safety of the workers, public and environment. Therefore, it is not trivial to focus enough effort in developing a methodology for Deterministic Safety Analysis that is able to predict realistic results that prove to the Nuclear Authority that the NPP operates within the safety margins during the proposed transient cases.

For this purpose, it is necessary to take advantage of the latest simulation tools, that have been able to apply more realistic physical models to obtain more accurate results, due to the development of the Computer Science and Technology. The fast development of the Computer Science allows to perform fast calculation procedures and an efficient management of significant amounts of data. This has facilitated the improvement of the numerical methods, discretization of system models and application of more detailed physics models and correlations. The result is a more realistic prediction of the derived variables, such as the Peak Cladding Temperature (PCT), that allow the reduction of the safety margin (see Figure 2.5). 
The possibility of predicting such realistic values with simulation codes supposed a new way for the Deterministic Safety Analysis. However, the Nuclear Authority requires the validation of new methodologies prior to their conventional use. Therefore, the possible options for the Deterministic Safety Analysis where documented in the corresponding Regulatory Guide of the USNRC.

Due to this fact, the Safety Analysis allows two approaches for the simulation methodologies, namely the BE and the Conservative Approach. Nevertheless, there are more aspects that influence the realistic or conservative features of a methodology. Table 2.5 summarizes the different conditions applied in the simulation for Deterministic Safety Analysis as required by the Nuclear Authority.

Table 2.5 Different approaches for the Deterministic Safety Analysis.

\begin{tabular}{|l|l|l|}
\hline $\begin{array}{l}\text { CONSERVATIVE } \\
\text { ANALYSIS }\end{array}$ & COMBINED ANALYSIS & $\begin{array}{l}\text { BEST ESTIMATE ANALY- } \\
\text { SIS }\end{array}$ \\
\hline $\begin{array}{l}\text { Use of conservative } \\
\text { computer codes with } \\
\text { conservative initial } \\
\text { and boundary condi- } \\
\text { tions. }\end{array}$ & $\begin{array}{l}\text { Use of BE codes combines } \\
\text { with conservative initial and } \\
\text { boundary conditions. }\end{array}$ & $\begin{array}{l}\text { Use of BE codes with whether } \\
\text { conservative or realistic } \\
\text { boundary and initial conditions } \\
\text { but, complementing the results } \\
\text { with the evaluation of the un- } \\
\text { certainties in the input data and } \\
\text { the uncertainties associated } \\
\text { with the models in the com- } \\
\text { puter code. }\end{array}$ \\
\hline
\end{tabular}

It must be accounted that the quantification of uncertainties is necessary when calculating realistic results. The BE approach predicts results of the derived variables that are relatively close to the real value. Due to this the quantification of the tolerance band associated to that predicted value will define in which cases the predicted value can be above (or below) the real value. This is important since in case of a conservative approach, there is always a wide enough margin separating the predicted value from the real value that tilts to the safety. Nonetheless, the $\mathrm{BE}$ approach can be so close to the real value, that the uncertainty inherent to the code and conditions of the simulation can predict a value trespassing the safety variable. The quantification of the uncertainty is necessary in order to evaluate how the proximity of the simulation results is close to the safety variable. Figure 2.5 shows the disposition of the safety margins according to the safety variable that is being evaluated. 


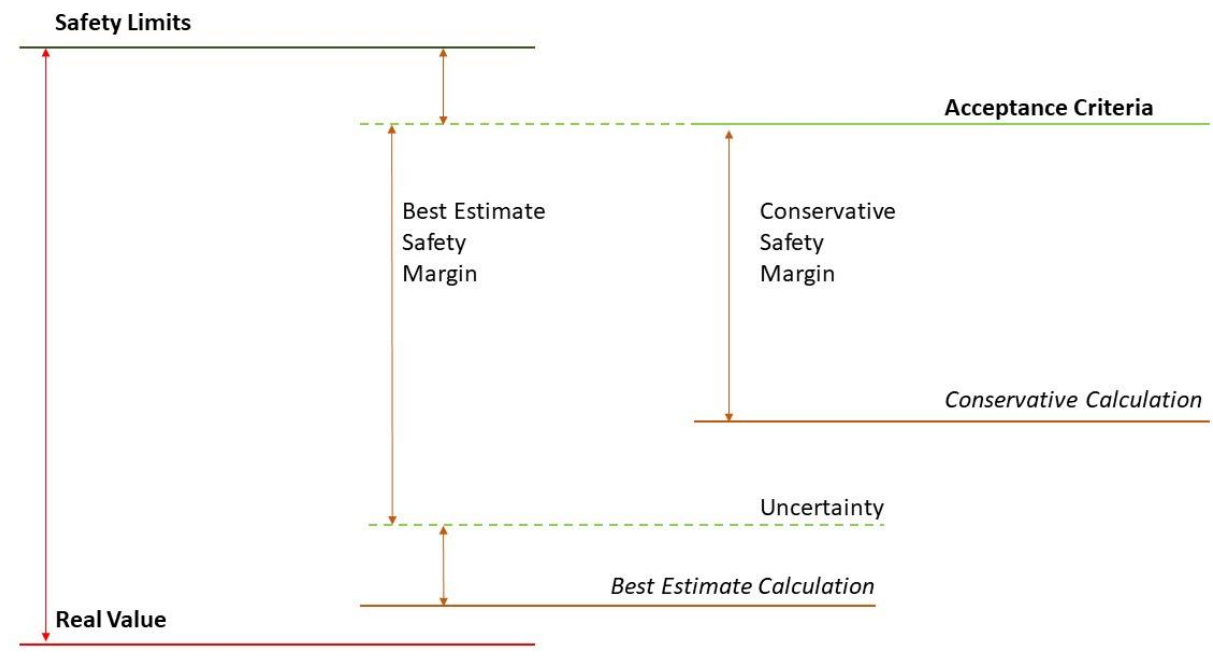

Figure 2.5 Scheme of the approaches to the safety margins assessment.

The opportunity in safety simulations presented by the commented development of the Computer Technology has been materialized in several projects undertaken by different institutions of the Nuclear Field. These projects intent to gather all the available physics involved in the phenomena of the transient events in a NPP and solve the corresponding equations within the different scales of the model.

Thus, the increase in the capabilities of computers together with the need of supporting and accelerating the improvement in the competitiveness of the nuclear industry have been captured in the framework of different projects that are providing the necessary simulation and modelling capacity for these purposes. These projects, such as CASL (Consortium for Advanced Simulation of LWRs), are the result of the common will of different public and private institutions. The aim of these projects is to develop a useful tool capable of model and simulate the behavior of the more relevant parts of a NPP at different scales and physics. Therefore, it is confirmed that the SOA in the field of the Nuclear Technology is related to the evaluation of the NPP behavior accounting all the physics at the necessary detailed level regarding 4 key points: neutron kinetics, thermalhydraulics, fuel performance and corrosion chemistry.

The Department of Energy of the USA expressed the relevancy of modelling and simulating as a useful tool for the Nuclear Technology in the CASL project (Energy Innovation Hub, 2010). CASL uses the multi-scale and multi-physics capabilities for tackling the safety analysis of LWRs. The two capabilities have to be combined in the challenges that CASL considers that are present in the evaluation of the most relevant 
transients in the operation of a NPP. Therefore, a proper combination of detail level and physics phenomena show good results in the evaluation of problems such as Pellet-Cladding Interaction (PCI) and Chalk River Unidentified Deposition (CRUD), but also in the prediction of relevant safety variables like Departure of Nucleate Boiling (DNB) and Peak Cladding Temperature (PCT).

Moreover, the projects headed to next generation Nuclear Reactors account the importance of the multi-scale and multi-physics approach. For instance, project NEAMS (Nuclear Energy Advanced Modelling and Simulation) (Versluis et al., 2013) develops a simulation toolkit for the analysis of the NPP operation accounting system level, core analysis, and fuel behavior. For this purpose, the toolkit is integrated in the corresponding platform in order to facilitate the data flow, pre- and post-processing. The toolkit accounts plant level analysis, pin resolved neutron kinetics, Monte Carlo analysis, neutron kinetics depletion, Cross Section preparation, thermal-hydraulics at low resolution, thermal-hydraulics at high resolution (i.e. Computational Fluid Dynamics, CFD), and the fuel analysis accounting microstructure, component aging and chemistry.

The investment in multi-scale and multi-physics analysis methods express the tendency of this way of evaluating the core behavior in order to obtain the sufficient realistic results for a safe and cost-efficient NPP operation.

Furthermore, such realistic results need the evaluation of the associated uncertainty in order to obtain the corresponding margins of the predicted values. The uncertainty is present at different levels of a core calculation methodology, for instance the uncertainty at the measurements of the introduced boundary conditions, or the propagation of the uncertainty along the numeric methods of the calculation codes. Therefore, when using $\mathrm{BE}$ tools or methodologies it is necessary to evaluate the margins of the predicted results, and thus an uncertainty and sensitivity analysis is required.

The importance of analyzing the uncertainty at the results of a simulation in safety analysis is expressed in different methodologies, such as the Code Scaling, Applicability and Uncertainty (CSAU) (Boyac et al., 1989). The CSAU methodology was validated by experienced companies of the nuclear sector such as FRAMATOME for the SB-LOCA safety assessment and accepted by the USNRC. This methodology includes the following relevant steps:

- Specify the transient scenario and select the safety variables to be analyzed.

- Identify and rank phenomena. This step includes evaluating the uncertainty sources and quantify its uncertainty in order to evaluate its propagation. For this step it is necessary to use whether the available scientific literature or the expert criteria.

- Perform the sensitivity analysis, i.e. evaluate how the uncertainty of the input variables are related to the uncertainty of the output target variable. 
Development and validation of a multi-scale and multi-physics methodology for the safety analysis of fast transients in Light Water Reactors

\subsection{The role of Sensitivity and Uncertainty Analysis.}

The possibility of using Best Estimate codes and Realistic Boundary conditions was the starting point for a Safety Analysis without excessive ranges of conservatism, that lead to over-dimensioned designs and operation strategies. However, it is duty of the Nuclear Technology not to compromise the safety while enhancing the accuracy of the results. For that purpose, the yielding of realistic results is complemented with the so -called Uncertainty and Sensitivity Analysis.

The uncertainty quantification (UQ) defines the boundaries in the predicted safety variable that are a result of the propagated uncertainty of the input variables through the code simulation. The UQ also defines the percentile that lays in a postulated Confidence Interval. The USNRC defines in its regulation that the UQ must meet the 95/95 criterion, that means that in the $95 \%$ of the cases, the predicted value lays in the confidence interval of the $95 \%$ of the mean value. In order to meet these requirements, the analyst undertake a statistical approach that evaluate the correlation between the uncertainty of the input parameters and the resulting uncertainty of the target output variable. Figure 2.6 is used to show a scheme of how the uncertainty in the input parameters result in the uncertainty of the output variable propagated through the code simulation.

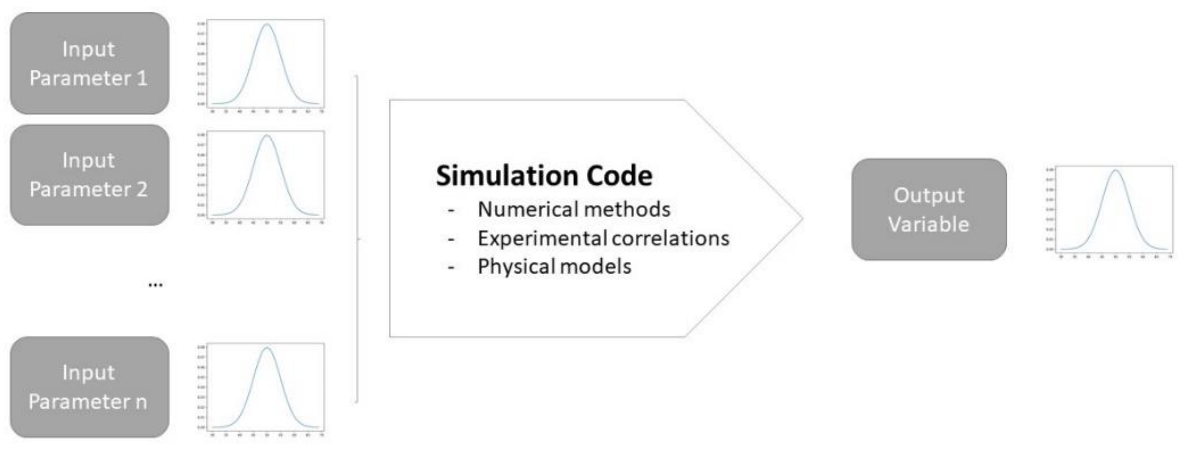

Figure 2.6 Uncertainty flow through the code simulation.

To proceed with the UQ it is necessary first of all to define and bound the uncertainty of the input parameters, and to know as well the which parameters are relevant to calculate 
the behavior of the output parameter. For example, it is known that the system pressure is a key parameter for the prediction of the MCPR (Minimum CPR), therefore it is necessary to account the uncertainty of the system pressure in a simulation of a transient, where the figure of merit is the MCPR. The scientific literature and gathered experiences, both industrial and experimental, are relevant sources for defining the uncertainty of the input parameters when performing the U\&S analysis.

On the other hand, to state the accomplishing of the statistical criteria, let us say the $95 / 95$, it is necessary to work with the proper sample size that gives consistency to the overall results. For that purpose, a sample of simulations is run, where the selected input variables are randomly varied according to their probability density function. The result is a distribution in the target output variable whose statistical parameters must meet the postulated criterion.

The reader must notice that the sample size will have a significant impact in the computational cost of the UQ. The research in efficient methods that bring about the U\&S analysis in an efficient way without compromising the required statistical criteria result in the current tools for such analysis.

\subsubsection{Background.}

BEPU methodologies are one of the most interesting points of the developments of the Safety Analysis. Nevertheless, the application of realistic calculations was introduced about 20 years after the use of Nuclear Technology, thanks to the Best Estimate codes. Back then, the state-of-the-art in core physics, numerical methods and computer technology limited the Nuclear Regulators to set conservative measures for the evaluation of the safety in the operation and design of Nuclear Reactor Cores. The large amount of uncertainty in such calculations forced to assume those margins, exchanging operational flexibility and cost-efficiency for Safety.

The results were observed after the long-term experience of NPPs, that revealed that power uprate was possible without compromising the safety of the plant. These power uprates were accompanied by enhanced and more realistic calculations, that yielded the different options existing currently (see Table 2.5). The purpose of performing more realistic calculations was to get beyond the limitations of the Conservative Approach. On the one hand, the Conservative Approach can cover the true behavior of the core during a simulated scenario by affecting the order of the event occurrence. On the other hand, this type of approach does not allow quantifying the safety margins, since the level of conservatism is not known.

The Conservative Approach was successfully overpassed in the decade of the 70s, however, the use of Best Estimate codes reduced the safety margin in a way, that accounting the effect of the uncertainty was necessary. The basis is the assumption of the fact that there are sources of uncertainty in the prediction of the safety variables that are introduced in the approaching of the problem. These uncertainties can propagate through the simulation core in process of calculating the core behavior. The result is that there exists 
Development and validation of a multi-scale and multi-physics methodology for the safety analysis of fast transients in Light Water Reactors

certain amount of uncertainty in the prediction of the safety variable. Therefore, if the use of more realistic methods for predicting safety variables reduce the margin of the order of approach to the real calculation (see Figure 2.5), the effect of the uncertainty could lead to exceed the real safety values without being able to know.

The development of the realistic calculations was materializing in the will of Nuclear Regulators to codify the requirements for such approach. On the other hand, the Nuclear Industry and Scientific field was internationally undertaking and effort with the aim of developing the Best Estimate Approach in codes that applied the available and known physics of the core in order to predict the evolution of key parameters that could restrict the operational limits of the plant. This task came along with the gathering of data that allowed the verification and validation of the BE codes.

These concerns have driven the efforts of the scientific community to tackle the problem. The objective of bounding the uncertainty and analyze its propagation until the predicted variable yielded the uncertainty quantification. Moreover, the use and development of statistics defined the way to correlate the uncertainty of the input and the output during the calculation procedure of the safety variables. The evaluation of how the input variables can influence in the prediction of the output variables, together with the quantification of such uncertainties resulted in the Uncertainty and Sensitivity Analysis.

The results of the international discussion about the relevancy of the use of Best Estimate simulation tools and the need of the analysis of the related uncertainty led in 2005 to the establishment of the Uncertainty Analysis Modelling meeting (UAM). This meeting organized by the NEA/OECD meant to form an Expert Group to endorse a workshop capable of planning the scope of future international research effort in the field of the Uncertainty and Sensitivity Analysis of the Nuclear Technology. The outcome of this international effort was to organize a benchmark work program made of different exercises in order to provide a solid basis of measured data that could be helpful for the validation and analysis of different methodologies regarding multi-physics and multiscale simulations.

\subsubsection{Concern in bounding the uncertainty and current experiences.}

One of the most relevant steps for evaluating the uncertainty in the target predicted variable is to bound the sources of uncertainty. First of all, it is necessary to difference between two types of uncertainty. The epistemic uncertainty makes reference to the lack of knowledge, in the case of core physics, in modelling the evolution of the parameters that we want of analyze. A model has the objective of predicting and quantify the reality, but these models approximate to the real behavior as a function of our understanding of all the physic processes that take place on int. The other type of uncertainty is the stochastic or aleatory uncertainty. This type makes reference to the probabilistic variability that is inherent to a process and it is assumed that it cannot be reduced by increasing the knowledge of the analyst in the modelled system. The amount of uncertainty in this case 
Chapter 2. State-of-the-art

is related to a probability distribution. Table 2.6 summarizes the main points of both types of uncertainty (Brun et al., 2011). 
Development and validation of a multi-scale and multi-physics methodology for the safety analysis of fast transients in Light Water Reactors

Table 2.6 Differences between stochastic and epistemic uncertainty.

\begin{tabular}{|c|c|c|}
\hline Features & Stochastic & Epistemic \\
\hline Representation & Class of possible outcomes & Single case \\
\hline Focus of Prediction & Event propensity & Binary truth \\
\hline $\begin{array}{l}\text { Probability Interpreta- } \\
\text { tion }\end{array}$ & Relative frequency & Confidence \\
\hline $\begin{array}{l}\text { Attribution of Uncer- } \\
\text { tainty }\end{array}$ & Stochastic behavior & Inadequate knowledge \\
\hline Information Search & Relative frequencies & $\begin{array}{l}\text { Patterns, causes and } \\
\text { facts }\end{array}$ \\
\hline
\end{tabular}

The kind of uncertainty governing the safety analysis in the core is the epistemic uncertainty. This is due to the fact that a certain lack of knowledge is reflected in the physical models, that come from the scientific experience. Moreover, the numerical methods applied to solve the equations governing the core physics also account certain error range in the solution. And in addition, it is necessary to account the error margin of the measurements, both in the physical parameters used as boundary and initial conditions, as well as in the geometrical values of the modelled system. To be more specific, since Regulatory Bodies like the USNRC appeals to identify the different types of epistemic uncertainty (Wieselquist et al., 2018), the following points describe three categories of this uncertainty type: Completeness Uncertainty, Parameter Uncertainty and Model Uncertainty. Nevertheless, this classification may be more useful for the Probabilistic Safety Analysis as reflected in the Probabilistic Risk Assessment defined by the NUREG document referend in (Siu et al., 2016).

The purpose of the quantification of uncertainty in the Deterministic Safety Analysis is to evaluate the propagation and limits of the lack of knowledge in the prediction of safety variables, such as the PCT, in order to bound an already realistic prediction. Uncertainty in this aspect is assumed as a key issue due to the fact that for the DSA it must be accounted the different uncertainties that come from a range of types and sources. A more useful classification of the uncertainty (Rotmans \& van Asselt, 2001) defines two features of it: Variability and Lack of Knowledge. Variability is the feature of the analyzed system that considers that the behavior can be different due to the inherent randomness of nature or value diversity. This feature can contribute to the lack of knowledge since due to the variability certain knowledge can be unattainable. On the other hand, the knowledge of a deterministic process can be incomplete. The lack of knowledge includes the inexactness, ignorance or observations that are unknown because they are practically immeasurable. Nevertheless, as Figure 2.7 depicts, it is possible to connect both features of uncertainty. 


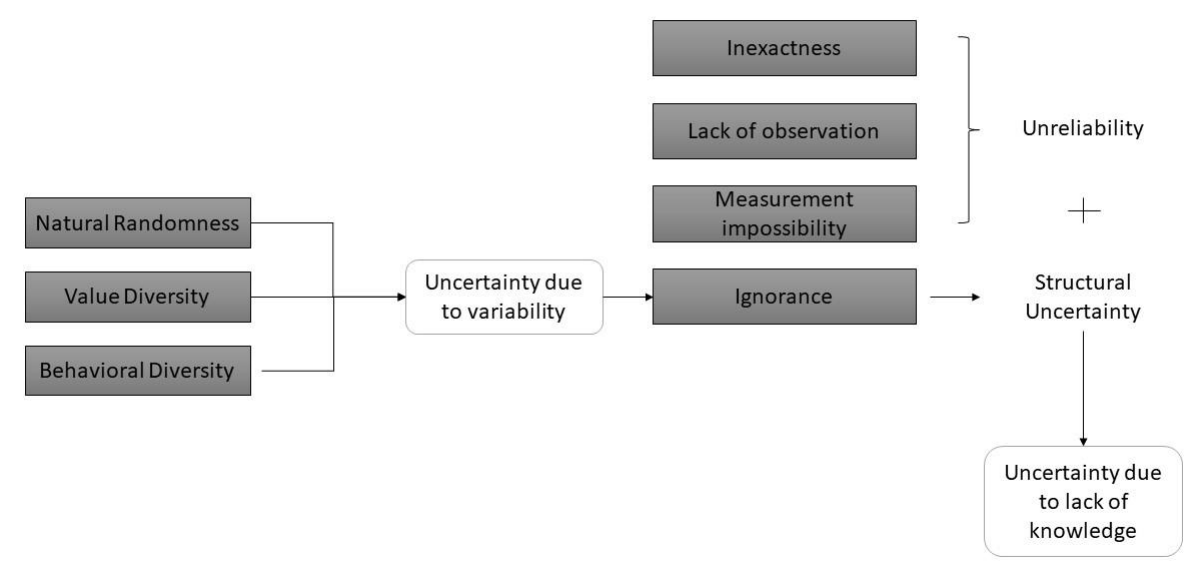

Figure 2.7 Features of the uncertainty.

The current understanding of the phenomena governing the core physics can be categorized then in three types of uncertainty (Funtowicz \& Ravetz, 1990): technical, methodological and epistemological.

The technical uncertainty comes from the quality of the data that describes the system, from temporal and spatial aggregation and from the lack of data. The methodological uncertainty comes from the analytical tools and the method used for analyzing the scenario. Finally, the epistemological uncertainty refers to the conception of the analyzed phenomena.

When modelling the core physics in a simulation tool, the uncertainty is bounded in the following categories:

a. Uncertainty in the simulation tool: Accounts approximations related to which terms are used in the field equations, uncertainties in the built in properties of materials and the assumption of the modelling of the fluid dynamics.

b. Uncertainties in the representation of the model: Accounts the effect of defining the control volumes where the solution of the problem is calculated. This effect depends on the uncertainty of the nodal distribution.

c. Uncertainty of the scaling of the model: The modelling of certain phenomena accounted in simulation code assume the uncertainty of scaling laws of the experimental data retrieved from benchmarking and applied to full scale plant models.

d. Uncertainty in plant conditions: Parameters to define the conditions of the model to be simulated come from plant measurements that include certain band of uncertainty. 
Development and validation of a multi-scale and multi-physics methodology for the safety analysis of fast transients in Light Water Reactors

e. Uncertainty of the user effect: Accounts the effect of the number and way in which each user influences the use of the simulation tool.

The initial BE and Uncertainty application based on the issue described in this section was made by the USNRC, gathering the methodology in the CSAU project. This lead was followed by several other countries that were up to approach this topic, like GRS, IPSN or ENUSA, among others.

In these methodologies, e.g. CSAU, the analysts provide in the so-called Phenomena Identification and Ranking Table (PIRT), the list of relevant phenomena affecting the safety variable to be evaluated. For instance, in a LOCA event, the safety variable would be the PCT, and the PIRT would list all the physical phenomena contributing to the evolutional behavior of the PCT. The reader must notice that the uncertainty in these phenomena will contribute to the uncertainty of the safety variable as output. Therefore, these input uncertainty will define the margins of the prediction of the safety variable, depending also in the statistical techniques used to evaluate this uncertainty and confidence margins. The input uncertainty will be printed, for example, in the coefficients used to model the behavior, which is based on experimental data. Another contribution is the uncertainty itself in the measurements attached to the experiment and the random behavior of the test undertaken to retrieve all the experimental data. In addition, the application of such physical correlations in the simulation code are affected by the nodal distribution of the model. This distribution must account the needed dimensions for evaluating the relevant physical phenomena of the event to be analyzed. This means that the calculation nodes must have the proper size to not diffusing the simulated physical behavior. On the other hand, the analysts must assume certain compromise to no incur in an unnecessary and prohibitive nodal mesh that affects the computational cost. All these parameters are accounted in the contribution to the uncertainty of the predicted variable.

The following step is to attach a statistical distribution to the uncertainty of the input variables. This is normally based on the scientific and industrial accumulated experience. The CSAU methodology use normal and uniform distributions.

Finally, it is necessary to use a statistical method for the evaluation of the propagation of the uncertainty from the input, through the simulation code and finally to the output safety variable. CSAU methodology does not use a standard method. The response surface approach (Khuri \& Mukhopadhyay, 2010) was proposed as statistical method. On the other hand, the GRS methodology (Glaeser, 2008) bases its statistical approach in the formula of Wilks. This formula sets an optimized number of simulations, sampled according to the uncertainty of the input parameters. The result is a sample of the output variable that generates a probabilistic distribution, based on the probabilistic distribution of the input variables. In that sense, the formula of Wilks is a useful tool to optimize the number of simulations to do, for a desired probability and confidence interval.

The formula of Wilks has revealed as a powerful technique to perform the Uncertainty Quantification, which nowadays is a necessary requirement to undertake Safety Analysis 
with a realistic approach. For this reason, this statistical method will be detailed in the following subsection.

\subsubsection{The theory of Wilks.}

The Wilks theory is grounded on the order statistics for covering the analyzed safety variables in a certain confidence interval. Since the IAEA standards do not specify the statistical methods for quantifying the uncertainty attached to the safety figures of merit, different organizations developed their own procedures. The German organization Gesellschaft für Anlagen- und Reaktor Sicherheit (GRS) was the first one in applying the formula of Wilks for such analyses.

The theory of Wilks (Wilks, 1941) is headed to answer the question about how large should be a sample in order to a proportion of it can be included between the desired tolerance limits. The target of this formula is to optimize a procedure of obtaining a probabilistic distribution of the output safety variable to be analyzed. Current Uncertainty Analysis undertaken in the Safety Analysis responds to the 95/95 criterion. This means that a percentile of the $95 \%$ of the sample must meet a $95 \%$ Confidence Interval. Equation 2.3 shows the formula of Wilks, where $\alpha$ is the percentile of the output distribution, $\beta$ is the confidence level of the output distribution, $N$ is the minimum number of code simulations and $\mathrm{p}$ is the order of the output population.

$$
\sum_{j=0}^{N-p} \frac{N !}{(N-j) ! j !} \alpha^{j}(1-\alpha)^{N-j} \geq \beta
$$

The order of the approach sets the number of code simulations that are expected to fall beyond the $\alpha$ percentile, $95 \%$ in the $95 / 95$ criterion.

For this postulated analysis, the Wilks formula sets the corresponding number of samples to be defined, i.e. the number of simulation cases. Accounting the uncertainty in input variables as well as the inherent uncertainty of a simulation tool, a number of code runs should be simulated. In order to have both distribution of the input variables and the output variable, for each calculation every input defined variable is varied simultaneously. The strength of this method is that the number of calculations needed to quantify the uncertainty in the output variable is independent of the number of uncertainty-adding input parameters.

One of the main concerns using statistical approaches for quantifying the uncertainty in the prediction of safety variables is to reduce the number of simulation runs. This concern led to the use of non-parametric approaches instead of the parametric ones. 
Development and validation of a multi-scale and multi-physics methodology for the safety analysis of fast transients in Light Water Reactors

The reason of this lays on the fact that a parametric sampling approach the number of samples, i.e. code simulations is dependent on the input parameters, which will be varied according to their uncertainty. Therefore, if there are several input parameters whose uncertainty is assumed to contribute to the uncertainty of the output variable the sample size of simulations will be large. In addition, the parametric approach is valid for normal distributions. The drawback of this sampling approach is that the computational cost can be prohibitive and additional effort must be done to verify that the Probability Density Function (PDF) of the response is of the normal type.

Conversely, the approach for non-parametric sampling achieves to reduce significantly the sample size. The reason is that the input parameters are sampled according to their uncertainty simultaneously. Hence, the number of samples will be independent of the number of input parameters. In addition, this alternative approach does not need to work with normal distributions only.

The method of Wilks, as well as other order approaches have been accepted currently by Nuclear Regulators such as the USNRC for the evaluation of the uncertainties in safety analysis. These methods allow meeting the 95/95 criterion for bounding the results of the prediction in safety parameters, reducing the computational cost of the analysis and hence improving the efficiency in Safety Analysis. Hence, the number of simulation is reduced to a more feasible number.

The number of simulations that form the sample is defined by Equation 2.4 and Equation 2.5 , for simple and double tolerance respectively.

$$
\begin{aligned}
& 1-\alpha^{n} \geq b \\
& 1-\alpha^{n}-n(1-a) a^{n-1} \geq b
\end{aligned}
$$

In these equations, $a$ is the unitary value of the percentile of the correspondent output distribution that lays in the unitary value of the confidence interval of $b$, for an $n$ sample size. The sample size $n$ increases with the values of $a$ and $b$, and also with the tolerance band, whether double or simple. Table 2.7Table 2.7 summarizes the number of samples needed for meeting the different statistical criteria for both simple and double tolerance limits. 
Table 2.7 Number of samples for different statistical criteria.

\begin{tabular}{|c|c|c|c|c|c|c|}
\hline \multirow{3}{*}{$\alpha(\%)$} & \multicolumn{6}{|c|}{$\beta(\%)$} \\
\cline { 2 - 7 } & \multicolumn{3}{|c|}{ Simple tolerance limit } & \multicolumn{3}{c|}{ Double tolerance limit } \\
\cline { 2 - 7 } & 90 & 95 & 99 & 90 & 95 & 99 \\
\hline 90 & 22 & 45 & 230 & 38 & 77 & 388 \\
\hline 95 & 29 & 59 & 299 & 46 & 93 & 473 \\
\hline 99 & 44 & 90 & 459 & 64 & 130 & 662 \\
\hline
\end{tabular}

For certain scenarios, there may be more than one $r$ dependent output variables to be analyzed. In these cases, it is necessary to modify the formula of Wilks (Guba et al., 2003) as Equation 2.6.

$$
\sum_{j=0}^{n-s \cdot r} \frac{n !}{(n-j) ! j !} \alpha^{j}(1-\alpha)^{n-j} \geq \beta
$$

At this point, and regarding table 1.5 it is necessary to introduce the discussion on one of the variations of the formula of Wilks (Macián-Juan et al., 2011). The discussion arises from the option of using one side tolerance band and second order formula as a two-side tolerance band of first order. In these cases, different works (Wieselquist et al., 2018), (Vedovi et al., 2012) uses as Table 2.7 describes, a sample size of 93 simulation cases for a 95/95 criterion. Nonetheless, there are studies (I. S. Hong et al., 2013), (I. Hong \& Connolly, 2008) that recommend in these cases a sample size of 146 cases, according to Equation 2.7.

$$
1+\alpha^{n}-2 \alpha^{n} \sum_{k=0}^{n} C_{k}\left(\frac{1-\alpha}{2 \alpha}\right)^{k} \geq \beta
$$

This thesis work will show the results of applying the suggested modification in a UQ in Chapter 3. 
Development and validation of a multi-scale and multi-physics methodology for the safety analysis of fast transients in Light Water Reactors

\subsubsection{Sensitivity Analysis.}

Every UQ is complemented in parallel with the corresponding Sensitivity Analysis (SA). The SA is a procedure for correlating the influence of the uncertainty of separated input parameters in the overall uncertainty of the output variable. The definition of this correlation values between the output safety variable and the inputs can explain which phenomena are more relevant for the prediction of such safety variable and which are not. This is useful not only for reducing computational and different costs of the analysis, but also helps to have a better understanding of the modelling of the nuclear behavior.

The correlation between variables defines how strongly are they related. For certain models or phenomena, it can be easily observed, in a qualitatively manner, how input and output variables are correlated. For instance, it is clear that in the analysis of pool boiling, there is a strong correlation between the surface temperature and the void fraction of the control volume. The correlation between both variables will quantify not only how much can vary the void fraction with a certain increase of the surface temperature but also, through the sign of the correlation value, if the void fraction increases or decreases with the increase of surface temperature. In this example can be an easy case for the sensitivity analysis, however, nuclear systems are of more complicated models in which the correlation between variables is not trivial to observe. Furthermore, many of these relation between variables of the models are non-linear, which increases the difficulty to observe the magnitude of the correlation and hence, the Sensitivity Analysis plays an important role.

Throughout the SA, it is possible to evaluate which parameters are relevant for the prediction of the target safety variable, which are not, and in which level of influence. This influence is evaluated by quantifying the correlation coefficients of the input parameters and the output parameter. There are several types of correlation coefficients (de Rocquigny et al., 2008) that have different ranges of application and for this reason, it is necessary to know which are the most relevant and useful for the SA in the analysis of the behavior of a NPP.

The Pearsons Coefficient is a renowned correlation coefficient based on the covariance of the two variables divided by the product of their standard deviations. Equation 2.8 shows the Pearsons Correlation Coefficient $(\rho)$ for two random variables of a population,

$$
\rho=\frac{\operatorname{cov}(X, Y)}{\sigma_{X} \sigma_{Y}}
$$

that in the case of a sample of size $n$ will be modified to Equation 2.9, named sample correlation coefficient $(r)$. 


$$
r=\frac{n \sum x_{i} y_{i}-\sum x_{i} \sum y_{i}}{\sqrt{n \sum x_{i}^{2}-\left(\sum x_{i}\right)^{2}} \sqrt{\sum y_{i}^{2}-\left(\sum y_{i}\right)^{2}}}
$$

Equation 2.9

The point of this formula is to set the strength of the correlation between two variables understood as a linear relationship. Coefficient values close to 1 or -1 show a strength correlation whilst a 0 -valued coefficient would mean no correlation at all. The sign of the coefficient sets whether the correlation is direct or reverse. Even though this correlation is very conventional and robust, as well as easy to implement, presents certain features that have revealed the need of using other methods. On the one hand, since it is dependent on the standard deviation it is quite sensible to dispersed values. On the other hand, the formula of Pearsons (Boslaugh, 2012) is only valid for variables having a linear relationship, therefore, its application lose consistency when treating variables that are non-linearly correlated.

A proposed solution for enhancing Pearsons correlation coefficients is to use the rank of the distribution of the input and output variables. The rank correlation coefficients do not seek the linear correlation but the monotonic correlation between two variables. A monotonic correlation must be understood as a relationship showing that when one of the variables increases the correlated one increases and vice versa, or behave conversely, i.e. when one increases the other one decreases. This fact has a wider range in setting the correlation between variables since observing the strength and direction of this correlation is less restrictive than observing their linearity. In addition, non-linear behavior between variables is much more expected in the core physics. The Spearman's correlation coefficients (Forthofer \& Lee, 1995) is a common tool in the Sensitivity Analysis in Nuclear Safety. The application of this coefficient had the task of ranking the variable distribution prior to set their correlation. Equation 2.10 shows the formula of Spearman for the ranked correlation coefficients.

$$
r=1-6 \sum_{i=1}^{n} \frac{D_{i}}{n\left(n^{2}-1\right)}
$$

Equation 2.10

Ranking the variables for the input and output distributions to be correlated means assigning levels to the data sets, from the highest quantitative or qualitative value to the lowest. Therefore, according to Equation 2.10, for the $n$ size of the sample, the correlation will account the $D_{i}$ difference of the rank between adjacent ranked variables.

On the other hand, there is another method for the SA based on the functional decomposition of the variance in sensitivity analyses. These methods are useful when the nonlinear and non-monotonic hypotheses are confirmed or accounted. The most common 
Development and validation of a multi-scale and multi-physics methodology for the safety analysis of fast transients in Light Water Reactors

method of this branch is the use of the Sobol Indices (Sobol, 1993). This method is based on decomposing the variance of the output target variable into the effects of the input variables. These methods add the possibility of measure the variance across all the space of the input, using this variance as a measure of sensitivity. Sobol indices represent the total variance as an additional effect of the variances of the input variables or combined effect of input variables. The decomposed expression of variance after the development of Sobol is defined in Equation 2.11.

$$
\operatorname{Var}(Y)=\sum_{i=1}^{d} V_{i}+\sum_{i<j}^{d} V_{i j}+\cdots+V_{12 \ldots d}
$$

In Equation 2.11, $i$ and $j$ represent different variables of the total $d$ input variables contributing to the output variable $Y$. This equation is complemented with Equation 2.12 and Equation 2.13.

$$
\begin{aligned}
& V_{i}=\operatorname{Var}_{X_{i}}\left(E_{X_{\sim i}}\left(Y \mid X_{i}\right)\right. \\
& V_{i j}=\operatorname{Var}_{X_{i j}}\left(E_{X_{\sim i j}}\left(Y \mid X_{i}, Y_{j}\right)\right)-V_{i}-V_{j}
\end{aligned}
$$

The terms $\sim i$ and $\sim i j$ would represent the exception of $i$ and $i j$ terms in the notation, and $E$ correspond to the statistical term of expected value. The different indices are obtained as the example used in Equation 2.14, for the first order indexes.

$$
S_{i}=\frac{V_{i}}{\operatorname{Var}(Y)}
$$

Equation 2.14

The indices are standardized by the total variance in order to provide the fractional contribution. The consequence is that the total contribution of the different order indices equals the unit value. This method can potentially show the contribution to the final uncertainty of the output variable of every input variable itself, and the uncertainty of the combination of different and simultaneous input variables. Usually, the application of this method includes the consideration of the sensitivity of single input variables, also called first order indices, and the sensitivity of pair-combined input variables, called interaction indices. Other approaches of this type of SA (Saltelli, 2002) include the effect 
of the so called total indices. The main drawback of this method is that increasing the accuracy and completeness of the SA can yield significant computational cost. This method applies for models having relatively few input variables influencing the uncertainty of the output variable. A model accounting a significant number of relevant input variables would mean large amounts of sensitivity indices to compute, decreasing the efficiency of the process and calling for an alternative method for the SA.

An alternative approach to the estimation of the sensitivity of the output variable to the variance of input parameters is the Deterministic Approach, which is based on estimating the partial derivatives of the analyzed model at a specific point. Even though the Forward Sensitivity Analysis can be applied to any model, they have the restriction of being computationally expensive.

\subsection{Multi-physics and multi-scale features.}

The methodology developed in this $\mathrm{PhD}$ work includes the multi-scale and multi-physics features for the safety analysis. This subsection specifies the aspects of these features.

First of all, it is necessary to explain that the multi-scale concept can refer the coupling of several scales to solve a problem, but it may be possible to apply multi-scale analysis without any coupling (Bestion \& Guelfi, 2005).

The multi-scale approach is performed in order to combine the efficiency of a macroscale simulation, due to the use of coarser meshes, and the detail level needed for the evaluation of specific parameters. For instance, the prediction of the local DNB must account the two-phase flow phenomena. Due to this, it is necessary to focus the simulation locally and hence, zoom in the scale.

The evolution of the Computer Technology allows year by year more detailed simulations, however, the computational cost calls usually for simplified models, if the scenario allows it. An ideal case would allow varying the scale according to the needs of the simulation, but nowadays the safety analysis assume more accessible solutions.

According to this, and regarding the methodology presented in this work, the solution adopted is to analyze the transient scenarios using different and separated scales, in a zoom progression from a coarser model to a finer one. From Figure 2.8 to Figure 2.11 it is shown the different scales where the simulation of the different physics of the core can be applied.

Figure 2.8 shows the modelling of the Reactor Pressure Vessel and the components that the user has considered as relevant. The modelling of the system is done usually in 1D meshes where the TH equations are adapted to such scale and dimensions. The results in codes like RELAP5, TRAC-BF1 or TRACE have been validated confirming that the scale of analysis meets the expectations of the accuracy of the predicted results. 
Development and validation of a multi-scale and multi-physics methodology for the safety analysis of fast transients in Light Water Reactors
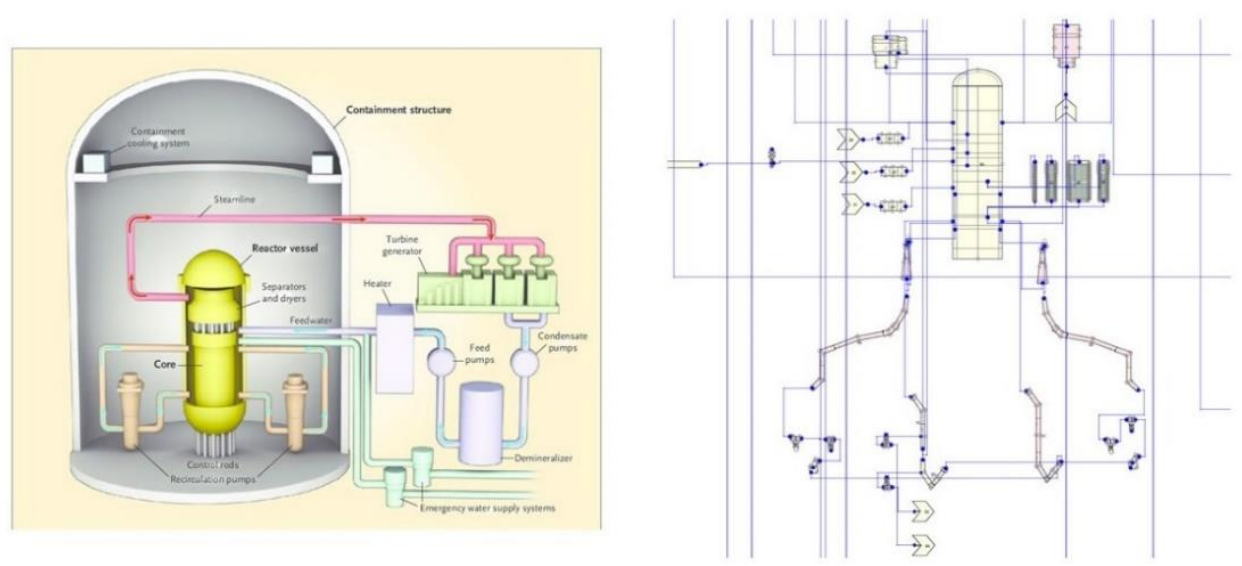

Figure 2.8 System scale modelled with SNAP tool for the simulation with TRACE code.

Moreover, a more detailed scale can be used, that allows tracking the predicted variables in different subcomponents. Figure 2.9 depicts the nodal mesh distribution of a reactor core, where the different fuel channels have been modelled. This distribution can be used for TH and NK codes like COBRA-TF and PARCS respectively, as well as for coupled $\mathrm{TH} / \mathrm{NK}$ codes.
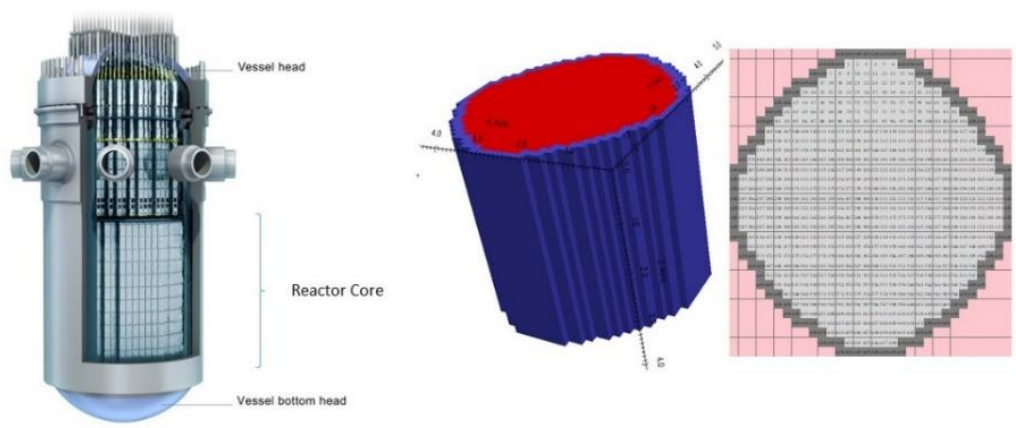

Figure 2.9 3D core modelled channel-by-channel represented with Paraview with the fuel channel layout. 
In addition, several local thermal-hydraulic phenomena are evaluated using a more detailed scale. The average values of a fuel channel modelled with lumped subchannels and heat structures can be insufficient for certain safety analysis. In these cases, is it possible to define the fuel assembly as an array of fuel coolant subchannels. Codes like COBRA-TF can provide models as the one depicted in Figure 2.10.
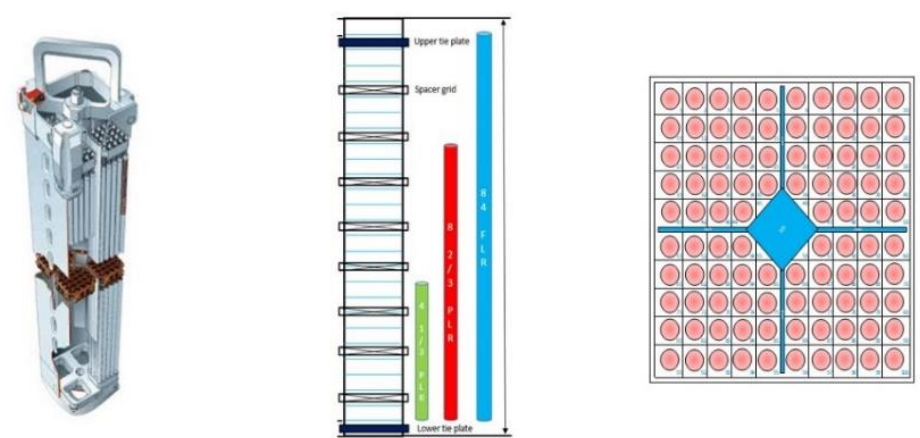

Figure 2.10 Axial and radial nodal distribution for a fuel assembly modelled for COBRA-TF.

Furthermore, the fuel thermo-mechanical behavior can be modelled in one single fuel rod. The behavior of the fuel pellet, gas gap and fuel cladding is predicted using codes such as FRAPTRAN. The fuel behavior codes can achieve a scale that includes the modelling of the rod springs and lower and upper plena, a detail level impossible to consider in system codes. Figure 2.11 shows the model for this kind of codes. 


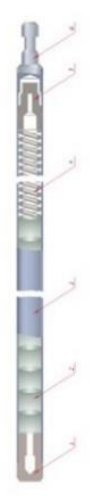

Figure 2.11 Fuel rod model for the thermo-mechanical analysis for FRAPTRAN code.

The multi-scale analysis for LWRs can be illustrated with four scales that can correspond to different simulation code types. This scale can be summarized in system scale, component scale, meso-scale and micro-scale.

- The models use different nodal distribution for adapting the scale level. These distributions can be in 0D, 1D, 3D or a combination of these ones.

- Adaptation of the methodology: System, core, channel, subchannel, pin. The use of a technique that couples different scales is beyond the features of this thesis.

- Adaptation of the methodology: System, core, channel, subchannel, pin. The use of a technique that couples different scales is beyond the features of this methodology. However, a more pragmatic view of the multi-scale analysis is applied in order to use smaller scale simulations. The methodology uses this multi-scale analysis to understand the basic phenomena by means of developing more physically based models or closure laws that may not be included in a macroscopic model.

The other relevant feature of the methodology is the multi-physics capability for the transient analysis. This feature is necessary since there are different physics involved in the behavior of the NPP, and also these physics can interact between each other. 
Accounting not only the different scales of the NPP but also the different physics as well as their interaction gives this methodology the capability of predicting BE results.

It is the aim of the methodology to account the different physics that evaluate the phenomena in the behavior of the NPP. The physics included in the methodology can be summarized as follows:

- Neutron Kinetics, NK: The source of the generation of the power in the reactor comes from the interaction and movement of the neutrons in the fuel and the rest of the core. Neutron Transport (W. M. Stacey, 2018) and Neutron Diffusion (W. M. Stacey, 1972) theories are useful physics analyzing the evolution of the neutron density and where are produced the different interactions. Moreover, the interactions of the neutrons along the operation of the NPP cause the evolution of the different materials present in the core. This evolution is expressed in the dependency of the Cross Sections on the burnup, i.e. depending on the fuel cycle or burnup step of the reactor, the Cross Section Set of each cell in the simulated model may vary. These variations will affect, for instance, to the capture or fission rate of the neutrons and hence, to the power rate. The neutron diffusion theory uses the sets of cross sections for simulating the performance of the NK part of the model, e.g. a channel-by-channel core model. However, it is necessary to use the neutron transport theory to calculate the homogenized few energy groups diffusion cross sections that will be used by the corresponding core simulator code.

- Thermal-hydraulics, TH: This field of the physics combines the thermodynamics, the fluid mechanics and the heat transfer in fluids and solids (Lahey \& Moody, 1993). The result is the evaluation of the coolant in the performance of the core transient. This analysis focuses on the phase change and how this affects to the evacuation of the heat generated by the nuclear fuel.

- Thermo-mechanics, TM: The behavior of the fuel is evaluated with the thermomechanics (Butkovich, 1997). This behavior analyzed regarding the evolution of the fuel cladding, the fuel pellet and the gap between them. This evolution depends on the heat exchange along the different parts of the fuel rod and the coolant. This field of the physics is significantly important for having an accurate prediction of the surface of the fuel cladding. Moreover, other safety parameters can be studied from this branch of the proposed methodology like the oxidation of the cladding, the mechanic deformation and the hydrogen deposition.

In addition, the Methodology accounts the interaction of different physics using the coupled feature. Coupling different field codes is a usual technique for a BE analysis (Ivanov et al., 1999). It must be accounted that when a single physics code is used stand alone, 
Development and validation of a multi-scale and multi-physics methodology for the safety analysis of fast transients in Light Water Reactors

the other physics must be introduced as a boundary condition. Using the coupling feature gives a more realistic prediction since it removes the user effect of introducing a boundary condition and enables the on-line treatment of the feedback effects between the physics. This methodology uses the coupled NK and Thermal-hydraulics for simulating transients at core level. Moreover, the limitations of TH at subchannel level from the surface of the cladding until the generation of power is complemented with the thermo-mechanics of the corresponding fuel performance code and vice versa. The combination of thermo-mechanics and TH of the Methodology leads to realistic predictions in fuel safety analysis.

Other fields can be included in the multi-physics analysis, but is beyond the scope of this thesis. For example, the CRUD deposition needs for the chemistry analysis of the different chemical species included in these phenomena.

Furthermore, there are different physical approaches for the different fields that are not accounted in the Methodology. The reason is that at the current point, the Methodology is focused on the available SOA tools, but other methods could be accounted for further work if the updating of the software used by the Methodology is necessary. These other approaches are the Stochastic Evaluation of the NK, such as Monte Carlo method, and the Computational Fluid Dynamics for the TH.

\subsection{State-of-the-art Codes.}

This section describes the main families of simulation codes used for the Safety Analysis.

\subsubsection{Neutron transport codes.}

The NK is the branch of the physics that analyzes the movement and interaction of the neutrons and the matter. The basis of this field is the transport theory of Boltzmann.

The NK is used to define the behavior of the nuclear reactors and the beams of neutrons used in test facilities, the industry and the medical field. The main point of NK for this thesis work is headed to analyze the static and dynamic behavior of LWRs.

The analytical tool for analyzing the core behavior is the diffusion equation, which is nothing more than an angular approximation to the Boltzmann equation. The use of the transport theory is not considered due to the high computational cost, whilst the diffusion theory provides results accurate enough in reactor cores that are relatively homogeneous, such as the LWRs. The derivation of the diffusion equation from the transport equation can be consulted in several references (Demazière, 2019).

The time-dependent and multigroup of the diffusion equation is shown in Equation 2.15. 


$$
\begin{aligned}
\frac{1}{v_{g}} \frac{d \Phi_{g}(\vec{r}, t)}{d t}= & -\nabla \vec{J}_{g}(\vec{r}, t) \\
& -\left(\Sigma_{a, g}(\vec{r}, t)+\sum_{g^{\prime}=1}^{G} \Sigma_{g^{\prime} \neq g}(\vec{r}, t)\right) \Phi_{g}(\vec{r}, t) \\
& +\sum_{g^{\prime}=1 g^{\prime} \neq g}^{G} \Sigma_{s, g^{\prime} \rightarrow g}(\vec{r}, t) \Phi_{g}(\vec{r}, t) \\
& +\chi_{g}(\vec{r}, t)\left((1-\beta) \sum_{g^{\prime}=1}^{G} v \Sigma_{f, g^{\prime}}(\vec{r}, t) \Phi_{g^{\prime}}(\vec{r}, t)\right. \\
& \left.+\sum_{k=1}^{K} \lambda_{k} C_{k}(\vec{r}, t)\right)
\end{aligned}
$$

Equation 2.15

In this equation, it is present the influence of the $k$ groups of precursors of delayed neutrons. Consequently, Equation 2.15 is complemented with Equation 2.16, which defines the concentration of the precursors group.

$$
\frac{d C_{k}(\vec{r}, t)}{d t}=\beta_{k} \sum_{g^{\prime}=1}^{G} v \Sigma_{f, g^{\prime}}(\vec{r}, t) \Phi_{g^{\prime}}(\vec{r}, t)+\sum_{k=1}^{K} \lambda_{k} C_{k}(\vec{r}, t)
$$

As observed, the neutron precursors concentration is dependent on the scalar flux, therefore, both Equation 2.15 and Equation 2.16 must be solved together.

In addition, Equation 2.17 shows the current net $\vec{J}_{g}$. This term can be calculated using the Fick's Law shown in Equation 2.17. 
Development and validation of a multi-scale and multi-physics methodology for the safety analysis of fast transients in Light Water Reactors

$$
\overrightarrow{J_{g}}(\vec{r}, t)=-D_{g}(\vec{r}, t) \vec{\nabla} \Phi_{g}(\vec{r}, t) \quad \text { Equation } 2.17
$$

Where $D_{g}$ is the neutron diffusion constant of group $g$.

The more relevant codes in this field of the physics are DYN-3D (Rohde et al., 2016), NESTLE (Turinsky, 1994), TRIVAC (Hébert, 1987), QUABOX (Langenbuch, 1984), PARCS (Downar et al., 2012), QPANDA (Smith, 1985) or VALKIN (Verdú et al., 1993). This thesis work pays special attention to PARCS, initially developed in Purdue University, since it is the most relevant code for Safety Analysis. The development of PARCS code is funded by the USNRC in order to obtain a reliable code managing the neutron diffusion equation as a part of the safety evaluation of the core behavior.

DYN-3D is a simulation tool for dynamic three-dimensional analysis of light water reactors. DYN-3D code was developed by Helmholtz-Zentrum Dresden-Rossendorf (HZDR) for the analysis of the soviet reactors type VVER and it is recommended by the IAEA for the analysis of such reactors. The code is modelled for simulating codes with the multigroup approach for both Cartesian and hexagonal geometries.

NESTLE code is a project developed by North Carolina State University (NCSU), Idaho National Engineering and Environmental Laboratory, Los Alamos National Laboratories and Oak Ridge National Laboratory. This code is designed for solving the diffusion equation with the multigroup approach using the nodal expansion method (NEM) for steady state and transient cases.

TRIVAC is a modular code developed by the Institut de Génie Énergétique of the Ecole Polytechnique of Montréal. This simulation tool allows the use of the finite element approach as well as the nodal methods for solving the $3 \mathrm{D}$ diffusion equation in cartesian and hexagonal geometries.

QUABOX-CUBBOX system was developed by Gesellschaft für Anlagen und Reaktor Sicherheit (GRS). These tools provide a detailed analysis of the core behavior based on 3D neutron diffusion codes for two energy groups. It is based on a coarse nodal mesh method that achieve a high precision for the radial node having the size of a fuel channel.

VALKIN code can use modal methods in order to integrate the neutron diffusion equation with the two-energy group approach. The modal theory uses the assumption that neutron flux can be expanded in terms of its lambda modes. The lambda modes are associated to the static configuration of the reactor core. It applies time updating strategies for the transient simulations. Hence, it considers a quasi-static nodal modal method.

\subsubsection{Thermal-Hydraulic codes at System Level.}

The system codes are used for the analysis of the TH at plant level. To be more specific, the system codes are used to model the cooling system of the reactor core. The evolution 
of the computer technology allows modelling in great detail the different mechanic systems of the plant and also the control systems. In addition, the research and development using benchmarks have improved the modelling of the thermal-hydraulic phenomena in these codes. Due to this, many system thermal-hydraulic codes can be considered BE, since they are capable of predicting realistic results.

The TH is the field of the physics in charge of analyzing the behavior of moving fluids that are being heated or cooled. It considers also the phase change if applies. This field joins the physics principles of fluid mechanics, thermodynamics and heat transfer. Therefore, this field includes the transport equations of mass, momentum and energy. The Navier-Stokes equation handle the analysis of the movement of the fluid, that can be applied to fluids with constant density $\rho$ and viscosity $\mu$. The results are shown in Equation 2.18 and Equation 2.19.

$$
\begin{gathered}
\nabla v=0 \\
\rho\left(\frac{\partial v}{\partial t}+v \nabla v\right)=-\nabla p+\rho g+\mu \Delta v
\end{gathered}
$$

Equation 2.18

Equation 2.19

At this point, it is necessary to add the first law of the thermodynamics, which considers that the change of the internal energy in a system equals the added heat to the system minus the work realized by the system. This will be introduced as an equation for the conservation of energy, shown in Equation 2.20. These three equations are the starting point of the analysis of the heated fluid mechanics (TH).

$$
\frac{\partial U}{\partial t}=\frac{\partial Q}{\partial t}-\frac{\partial W}{\partial t}
$$

TH codes were designed for the engineered safety systems. Afterwards, The USNRC introduced in its Federal Regulation Codes the requirements for conservative assumptions in the simulation of transient scenarios. These requirements are summarized in the Appendix K to Part 50 of the CFR10 (USNRC, 2017). This documentation headed the codes to assume conservative assumptions in the input models and conservative boundary and initial conditions in order to assure a conservative prediction regarding the analyzed safety variables.

At the first stages of the development of the thermal-hydraulic codes, they used to use the Homogeneous Equilibrium Model (HEM), this model considers the fluid to be in thermodynamic equilibrium for the two phases and hence, only three balance equations are needed, i.e. mass, energy and momentum. Nonetheless, the thermal-hydraulic codes 
Development and validation of a multi-scale and multi-physics methodology for the safety analysis of fast transients in Light Water Reactors

have evolved to consider the two-phase flow, accounting the balance equations for both, having then at least, 6 balance equations. In addition, the balance equations need of the constitutive equations in order to obtain a system with the same number of equations and variables to solve. Whilst the HEM model only uses two constitutive equations being the heat transfer relations at the wall and the friction loss, the two-phase flow analysis uses at least 7 constitutive equations.

It must be accounted that the proposed methodology uses two kinds of thermal-hydraulic codes: System and subchannel codes. There are significant facts that show certain differences in the performance of both codes.

The so called system or plant codes are able to model the relevant components of the primary and secondary cycles of a LWR. Usually these components are modelled in 1D and use empirical correlations to simulate their behavior. The components can be pumps, valves separators and pipelines and so forth. Nevertheless, these codes, e.g. TRACE, have the option to model 3D components such as the vessel, where the fuel channels are located.

The system thermal-hydraulic codes started modelling the thermal-hydraulic behavior of the nuclear reactors. The development of computational technology and nuclear technology have enhanced such codes to the point of being capable of modelling several components with a significant level of accuracy. In addition, the different validation and verification procedures have contributed to develop system thermal-hydraulic codes able to predict realistic results.

The USNRC has invested in the development of different licensing system codes such as TRAC-B (Miro et al., 2016), TRAC-P (Jenks \& Martinez, 1988), RELAP5 (Schultz, 2003) and RETRAN (Paulsen, 2014). Afterwards, the USNRC integrated the TRAC-B, TRAC-P and RELAP5 in the TRACE (Bajorek, 2011) code. On the other hand, the RETRAN code evolved to RETRAN-3D and RELAP5 to RELAP5-3D and RELAP7. We can find a similar scenario in the European industry. FRAMATOME, the French Atomic Energy Commission (CEA), EDF and the French Nuclear Safety Institute (IRSN) developed CATHARE (Robert et al., 2003) for the PWR analysis. The ATHLET (GRS, 2019) code was developed by GRS.

System codes usually model the plant components in 1D. Certain special components like the vessel can be modelled in primitive 3D meshes. TH codes allow this three-dimensional nodal distribution since models in 1-D usually introduce loss of information in the calculation. Therefore, 3-D modelling is an optimal nodal distribution for such components with complex flow behavior.

TH codes use different features to simulate each of the physics that take place in the reactor during the simulated conditions. Hence, every system code has to account on the one hand a NK model and a heat transfer model. This is necessary in order to model the generation and transport of heat along the reactor core. Moreover, the code must have a 
hydrodynamic model of the components in order to account the phenomena of the inner fluids.

Therefore, regarding the need of using a NK model, the available options in TH codes usually are: Point Kinetics, NK in 1-D or NK in 3-D. The features of the hydrodynamic models are based on whether they use single- or two-phase flow. The TH codes will solve the conservation equations for mass, energy and momentum.

TRACE is the System code in which the methodology of this thesis project is based. TRACE code implements TH models with balance equations for mass, energy and momentum for liquid and vapor phase. Regarding the NK, TRACE uses a Point Kinetics or a 1-D model and, in addition, can be coupled to a NK code for simulating the 3D neutron diffusion analysis. The TRACE code in its different versions has been provided in the framework of CAMP (Code Applications and Maintenance Program) in which the research group participates.

\subsubsection{Thermal-Hydraulic codes at Subchannel Level.}

TH subchannel codes can be considered as a hybrid code of CFD codes and System codes. The spatial meshing methods are analog to the initial CFD codes that used structured 3-D meshes. These codes are focused on the behavior of the fuel assemblies, modelling them in a subchannel mesh. Notice that the mesh cell can be whether the coolant volume enclosed by 4 fuel pins, or the coolant volume surrounding one fuel pin. The main advantage of these codes, in comparison to the CFD codes, is the calculation speed to solve the simulation problem. This advantage comes mainly from the fact that $\mathrm{TH}$ Subchannel codes do not account all the physics regarding turbulent mass flow, and that the two-phase flow models are adapted 1-D models. In addition, the approximation to the two-phase flow models is enough for simulating all the possible boiling regimes.

A significant part of the TH Subchannel codes come from the same original code, COBRA. COBRA (Avramova \& Ivanov, 2015) was developed by Pacific Northwest Laboratories for the analysis of the coolant behavior in fuel rod arrays. Several TH Subchannel codes have been developed from this starting point. The most relevant are the WCOBRA-TRAC from Westinghouse, the COBRA-TF from NCSU, the F-COBRA-TF from AREVA and VOPRE, developed by EPRI. Moreover, the KIT has developed the SUBCHANFLOW code focused on the analysis of fuel assemblies of PWR. The work developed for this thesis used the CTF-UPVIS code, which is the COBRA-TF version of ISIRYM.

\subsubsection{Coupled Features: Thermal-Hydraulics and Neutron Kinetics.}

The target of coupling NK (NK) and Thermal-hydraulics (TH) is including the 3-D kinetics in in the TH code in order to give the corresponding feedback to the TH code derived from the NK code. It is widely demonstrated that coupled codes TH/NK are the 
Development and validation of a multi-scale and multi-physics methodology for the safety analysis of fast transients in Light Water Reactors

most appropriate for the simulation of asymmetric transients where the evolution of coupled codes for simulating certain transient cases regarding the Design Basis in PWR, and mostly BWR.

The presented thesis work is focused in the use of coupled models of TRACE/PARCS and CTF/PARCS (Abarca et al., 2016). For these cases, the coupling methodology is similar and therefore, the explanation will be referred only for the CTF/PARCS coupling scheme.

The coupling scheme is known as time-dependent explicit since the NK and TH equations are not solved at the same step using the same scheme. Both codes will only exchange the necessary variable values to supply each other the boundary conditions for the corresponding time step. Each of the coupled codes solves its equation and the convergence for both has to be assured at each time step. The control of the convergence is done by the coupling control advance scheme.

\subsubsection{Fuel performance codes.}

In general terms, the fuel behavior analysis is done in two steps: the steady state simulation and the transient cases. The reason of this procedure comes traditionally from computational limitations. However, many benchmarks use this procedure, undertaking steady state tests and transient tests. These experiments use a fuel rod coming from an operation period in a nuclear reactor, where it has achieved the target burnup after a quasi-stationary operation.

This procedure usually has significant restrictions for the fuel relevant problems. This two-step-approach requires the coupling between both analyses. The coupling scheme can be difficult and add an extra of uncertainty depending on the compatibility of both codes. Nevertheless, besides these limitations, the experiences with these codes showed that it is difficult to implement in a unique code a single model structure that is able to manage both transient and steady state simulations. This is caused by the different features of the used models and the equations to be solved.

The USNRC has two reference codes that currently maintains: FRAPCON (Geelhood, Luscher, \& Beyer, 2011) and FRAPTRAN (Geelhood, Luscher, Beyer, et al., 2011). FRAPCON is used for the fuel analysis in steady-state and FRAPTRAN for the transient analysis.

The suite FRAPCON/FRAPTRAN allows using the results of FRAPCON as initial conditions to simulate a case with a known burnup in FRAPTRAN.

In a similar way, EPRI has developed two codes: ESCORE and FREY for the steadystate and transient calculations respectively. The ESCORE code is focused in the core loading for quasi-stationary cases, based on finite differences. ESCORE is licensed by the USNRC and uses average burnups until $50 \mathrm{GWd} / \mathrm{MTU}$. On the other hand, FREY is focused on the thermo-mechanical analysis of the fuel behavior in transient cases. FREY 
is based on finite elements. As it happens for the suite FRAPCON/FRAPTRAN, ESCORE can pass the initial conditions of a certain achieved burnup to FREY.

During the last years, the evolution of the fuel assemblies design and the increase in the use of the fuel assemblies themselves (reflected in higher burnups) has revealed a necessity of a reliable analysis capability of the transient and steady-state simulations. Due to this, it is a target for the fuel behavior code developers to design an integrated code including the steady-state and transient analysis. Therefore, EPRI works currently in the development and maintenance of FALCON, a fuel behavior analysis code including transient and steady-state analysis.

On the other hand, Idaho National Laboratory develops the application of finite elements to different fuel models in the advanced fuel behavior analysis code BISON. This code solves the thermo-mechanics and species diffusion equations in 1-D, 2-D and 3-D problems. Moreover, BISON is included in the MOOSE platform, which allows solving efficiently problems with high computational requirements. BISON includes high detailed models to describe the thermal properties depending on temperature and burnup, on deformation and cladding expansion, on fission products and so on. Consequently, it can be said that current code developments are focused in more complete analysis capabilities.

Among the different codes presented regarding the thermo-mechanical analysis of the fuel behavior, this thesis work is focused in the suite FRAPCON/FRAPTRAN. These codes are supported by the USNRC and are used currently for licensing and fuel reload in NPPs. These codes include models for the heat transfer through the fuel pellet and the cladding, models for the plastic and elastic deformation, models for the Pellet-Cladding Interaction (PCI), Fission Gas Release (FGR) models and so on. In addition, FRAPCON/FRAPTRAN count on material properties libraries for the fuel structural materials and coolant.

\subsubsection{Sensitivity and Uncertainty codes.}

Performing an Uncertainty and Sensitivity Analysis requires the application of three main features: Sampling according the provided input uncertainty, statistical analysis for the propagation of the uncertainty from input to output variables and sensitivity analysis of the correlation between the variations in the input and the output variables. There are currently solid developed tools that are able to realize such features. One of the most extended toolkits is DAKOTA, from Sandia National Laboratories.

These tools present different options for random sampling, i.e. provide a sample of a predefined size in which each member of the sample varies from another varying the selected input data. Tools like DAKOTA use the Probability Density Functions of the selected input variables provided by the user, and generates samples according to them. The user is able to selected different sampling techniques of the state-of-the-art like the Simple Sampling or the Latin Hypercube (LHC). By means of statistical analysis, these tools can give as result the mean value, standard deviation, skewness and so forth. In 
Development and validation of a multi-scale and multi-physics methodology for the safety analysis of fast transients in Light Water Reactors

addition, tools like DAKOTA provide the user with the option of applying different correlation coefficients like Pearsons or Spearman, or even different sensitivity analysis tools like the Sobol indexes. The use of such toolkits allow an efficient and complete analysis that is essential for a Best Estimate Plus Uncertainty analysis. 


\section{Chapter 3}

\section{Description of the methodology}

At the early stages of the deterministic safety analysis, the way to approach the evaluation of the acceptance criteria was made with rigorously conservative simulation schemes. These schemes where applied to simulate the Anticipated Operational Occurrences (AOO) and the Design Basis Accidents (DBA). The reasons of such conservative measures were no other than the computational cost of modelling components and so forth, and the difficulty of modelling the complicated physics governing the behavior of the core. Nevertheless, the advances in computational tools and the buildup of experimental data led to a further approach, i.e. the BE approach.

The scope of this thesis work includes the evaluation of the safety variables according to the corresponding Regulatory Organization by simulating the proposed transient scenario that may lead to AOOs and DBAs, as well as transient cases that take place in normal operation such as the shutdown of the NPP. The rest of the possible scenarios are beyond the scope of this work.

Nowadays, the Deterministic Safety Assessment is moving to assume the BE approach quantifying the uncertainties versus the Conservative approaches. The reasons can be summarized as follows.

1. The BE approach calculates more realistic results regarding the plant behavior. Moreover, this approach gives support to identify the relevant safety parameters as well as allowing a realistic comparison with the acceptance criteria.

2. It is possible that conservative approaches lead to unrealistic timescales or that it excludes certain physics phenomena. 
Development and validation of a multi-scale and multi-physics methodology for the safety analysis of fast transients in Light Water Reactors

3. The use of any conservative approach usually does not define the real margins of acceptance criteria. Operational Flexibility (Ponciroli et al., 2017) for instance could use of a BE approach for accounting real margins.

4. For the safety analysis of AOOs, a BE approach plus the corresponding quantification of the uncertainties can avoid the use of restrictive limits and set points. This fact leads to provide Operational Flexibility and will reduce an unnecessary use of SCRAMs.

Table 3.1 summarizes the possible occurrences according to different parameters according to the Safety Standards of the IAEA.

Table 3.1 Classification of occurrences in a NPP.

\begin{tabular}{|l|l|l|l|l|}
\hline $\begin{array}{l}\text { Occurrence } \\
\text { (1/reactor-year) }\end{array}$ & Characteristics & $\begin{array}{l}\text { Plant } \\
\text { State }\end{array}$ & Terminology & $\begin{array}{l}\text { Acceptance } \\
\text { criteria }\end{array}$ \\
\hline $\begin{array}{l}1.0-0.01 \text { (Ex- } \\
\text { pected over the } \\
\text { lifetime of the } \\
\text { plant) }\end{array}$ & Expected & AOOs & $\begin{array}{l}\text { Anticipated tran- } \\
\text { sients frequent } \\
\text { faults, incidents } \\
\text { of moderate fre- } \\
\text { quency, upset } \\
\text { conditions, abnor- } \\
\text { mal conditions }\end{array}$ & $\begin{array}{l}\text { No addi- } \\
\text { dional fuel } \\
\text { damage }\end{array}$ \\
\hline $\begin{array}{l}\text { 0.01 }- \text { chance greater } \\
\text { than 1\% over the } \\
\text { lifetime of the } \\
\text { plant }\end{array}$ & Possible & DBAs & $\begin{array}{l}\text { Infrequent inci- } \\
\text { dents of moderate } \\
\text { frequency, limit- } \\
\text { ing faults, emer- } \\
\text { gency conditions }\end{array}$ & $\begin{array}{l}\text { Not radio- } \\
\text { logical con- } \\
\text { sequence at } \\
\text { all or no ra- } \\
\text { diological } \\
\text { impact out- } \\
\text { side the ex- } \\
\text { clusion area }\end{array}$ \\
\hline $\begin{array}{l}0.0001 \text { 000001 } \\
\text { (Chance less than } \\
1 \% \text { over the life- } \\
\text { time of the plant) }\end{array}$ & Unlikely & $\begin{array}{l}\text { Beyond } \\
\text { DBAs }\end{array}$ & $\begin{array}{l}\text { Faulted condi- } \\
\text { tions }\end{array}$ & $\begin{array}{l}\text { Radiological } \\
\text { consequence } \\
\text { outside the } \\
\text { exclusion } \\
\text { area within } \\
\text { limits }\end{array}$ \\
\hline $\begin{array}{l}<0.000001 \text { (very } \\
\text { unlikely to occur) }\end{array}$ & Remote & $\begin{array}{l}\text { Severe ac- } \\
\text { cidents }\end{array}$ & $\begin{array}{l}\text { Faulted condi- } \\
\text { tions }\end{array}$ & $\begin{array}{l}\text { Emergency } \\
\text { response } \\
\text { needed }\end{array}$ \\
\hline
\end{tabular}


From the early days until the present time, there have been developed different methodologies established by the International Atomic Energy Agency (IAEA) that have been adopted by the Nuclear Authorities from every state. Table 3.2 shows the 4 options developed at the moment. These options are valid for proving that the NPP is able to manage the safety operation after the event meeting the acceptance criteria. The more advance option is option 4, which includes realistic analysis combined with the Probabilistic Safety Analysis. However, this option is no used widely nowadays.

Table 3.2 Options for the different combinations regarding the code accuracy and the accuracy of initial conditions.

\begin{tabular}{|c|l|l|l|}
\hline Option & Computer code & $\begin{array}{l}\text { Availability of } \\
\text { systems }\end{array}$ & $\begin{array}{l}\text { initial and } \\
\text { boundary condi- } \\
\text { tions }\end{array}$ \\
\hline 1. Conservative & Conservative & $\begin{array}{l}\text { Conservative as- } \\
\text { sumptions }\end{array}$ & $\begin{array}{l}\text { Conservative in- } \\
\text { put data }\end{array}$ \\
\hline $2 . \quad$ Combined & BE & $\begin{array}{l}\text { Conservative as- } \\
\text { sumptions }\end{array}$ & $\begin{array}{l}\text { Conservative in- } \\
\text { put data }\end{array}$ \\
\hline $3 . \quad$ BE & BE & $\begin{array}{l}\text { Conservative as- } \\
\text { sumptions }\end{array}$ & $\begin{array}{l}\text { Realistic plus } \\
\text { Uncertainty }\end{array}$ \\
\hline $4 . \quad$ Risk informed & BE & $\begin{array}{l}\text { Derived from } \\
\text { Probabilistic } \\
\text { Safety Analysis }\end{array}$ & $\begin{array}{l}\text { Realistic input } \\
\text { data plus uncer- } \\
\text { tainty }\end{array}$ \\
\hline
\end{tabular}

The proposed methodology focuses in the third option. That means that it will consider BE codes, conservative assumptions for the availability of systems and realistic input data with the corresponding uncertainty.

As chapter 1 explains, $\mathrm{BE}$ codes make use of all the possible physics that are involved in the core behavior. Moreover, these codes use the corresponding feedback of the different fields of the physics by means of the use of coupling schemes. Therefore, the result derives in the most realistic predictions of the safety variables. Furthermore, these results are enhanced using realistic input data, and consequently, the uncertainty must be accounted, in order to give the Confidence Interval of the predicted safety variable. This is done due to the fact that such realistic assumptions in the simulation procedures reduce the margin between the predicted safety variable to the corresponding safety limit, and hence (see Figure 2.5), it is necessary to predict the statistical boundaries according to certain statistical criteria, in order to check if those limits overpass the safety limits. In general terms, the statistical criterion proposed by the Regulatory Bodies is the 95/95 criterion, i.e. that the Confidence Interval of $95 \%$ is meet by the $95 \%$ of the sample. 
Development and validation of a multi-scale and multi-physics methodology for the safety analysis of fast transients in Light Water Reactors

The conservative approach was defined in the early stages of the safety analyses. According to the capabilities of the computational tools at that time, the uncertainties of the model and simulation where assured by specifying the most unfavorable conditions in regards of the acceptance criteria of the analyzed event.

According to Table 3.2, both options 1 and 2 are considered conservative approaches. The current conservative approach both initial and boundary conditions, as well as simulation models are defined conservatively. The assumption of such unfavorable conditions has proven that the predicted safety parameters are within the safety limits and ensures as well, that any other transient of the category will not exceed the acceptance criteria.

\subsection{Advantages of the BE approach.}

The causes of the introduction in safety analysis of BE approaches can be summarized in two parts: on the one hand the advance in core physics, its increase in knowledge and the buildup of experimental and operational data and experience. On the other hand, the development of the computer technology, that allows finer characterization of the real designs and large amounts of calculations. These facts yield in more accurate predictions of variables, that meet the experimental results satisfactorily. In the proposed methodology, the user can apply conservative assumptions depending on the selected boundary conditions, the simulation tools and scale analysis. However, according to the SOA of the Safety Analysis, it has been a priority to adapt this methodology to the BE simulation tools as well as the feature of accounting the flexibility of the analysis that allows varying the scale and the physics used. It will be on the hands of the user to define and apply the corresponding strategy for this methodology in the target scenarios to be analyzed.

In addition, the conservative approaches showed issues and even contradictions regarding safety analysis. These aspects can be described with a couple of examples. A more conservative approach will consider the uncertainty of the thermal core power. This means usually a higher thermal power than the nominal. According to this, a higher steam generation has to be assumed. The overestimation of the core power yields larger amounts of steam-water mixture generated in the core. The amount of steam can influence in a slower depressurization ratio in case of a postulated small break in the steam line. Such slow depressurization will prevent a faster deterioration of the heat removal capacity of the fuel rods. Therefore, the prediction of the safety variable of the Peak Cladding Temperature (PCT) may not be conservative. Another example can be showed by accounting a reduced interfacial shear between steam and water that leads to higher cladding temperatures in the upper part of the core. Nevertheless, the conservative approach in this aspect yields an optimistic estimate for the refilling and reflooding time since it will seem that there is more water in the primary loop that actually is. 
For this reason, a BE approach is needed, due to the fact that it could prove that some safety issues can be masked by the conservative assumptions. Hence, even in a conservative approach for licensing, it is necessary to ensure safety by using a BE approach.

Therefore, the deficiencies of a conservative approach are solved by an approach that uses BE codes and accounts the uncertainties attached to the model and lack of knowledge of the parameters of design and boundary conditions. This approach is known as BE Plus Uncertainty (BEPU) (Vedovi et al., 2012). The main advantages of this method are as follows:

a. It identifies and locates the most important safety issues.

b. This approach yields more realistic predictions of the physical behavior of the core.

c. Provides data of the existing margins between the predicted safety variables and the acceptance criteria.

The BE approach needs of simulation tools that account all the physics governing the behavior of the modelled component of the reactor. This comes along with gathering enough information that assures that all the relevant phenomena are taken into account in the model, or that their effects are bounded.

The fact is that when the BE code predicts safety variables close to the safety margins of the acceptance criteria, the uncertainties must be bounded to the analysis. It must be accounted that the combined action of realistic assumptions in the boundary and initial conditions plus the use of accurate and realistic computer tools must be complemented by the uncertainty for the assumed plant conditions and the code models. The purpose is that the predicted results do not trespass the safety limits. Usually, regulatory guidelines of the corresponding Nuclear Authority propose that the predicted results will not exceed the safety limits with a probability equal or greater than the $95 \%$. This requirement comes from the fact that it is not possible to assure the $100 \%$ of probability, and that the $95 \%$ is a common and conventional value standardized in several regulatory practices, that counts on large experience.

According to the different Regulatory Guidelines, it is normal to observe that the $95 \%$ probability of the simulated cases must fall in a confidence interval of the $95 \%$, which is the 2-sigma criterion.

In BEPU methodologies, it is highly recommended to use the Phenomenon Identification and Ranking Table (PIRT). This is a way to account the experts' criteria that, throughout experience, have evaluated and ranked the relevancy of different phenomena according to the postulated event to be analyzed. In this process for the quantification of the uncertainty, a sufficiently large sample of cases must be simulated, where the relevant input variables for the uncertainty of the safety output variable are varied according to their statistical distribution. 
Development and validation of a multi-scale and multi-physics methodology for the safety analysis of fast transients in Light Water Reactors

Another main feature of the BEPU approach is the use of the so called BE codes. For BE codes it is important to have a sufficient experimental data or operational data in order to validate the capabilities and their levels of qualification. The assessment of the capacity of the BE code to predict reliable values is done by comparing the predicted results against real data. The more extensive the assessment is the more confidence the code will have. Furthermore, Nuclear Authorities like USNRC, detail the procedure to assure the Quality Assurance of a simulation code (Odar, 2000). This documentation sets different steps to certify the simulation tool with the corresponding quality standards in a procedure that includes third party source code review, the requirement of documenting the code description, its physics and the user manuals, the validation of the modelled physic phenomena against benchmark data, the validation against NPP operation data and the verification against validated codes.

The codes for BE analysis are classified as follows:

a. System Thermal-Hydraulic codes: As commented in the state-of-the-art subsection, system Thermal-Hydraulics codes make reference to those computational tools able of modelling different plant components, even separately.

b. Core physics codes: Core physics simulation tools are focused in the calculation of the neutron flux, the criticality of the core, fuel management and core following and power distributions.

c. Component specific or phenomenon specific codes: Component or phenomenon specific codes are specialized in the analysis, whether in steady state or transient, of specific components such as the core, the fuel rod and so on. On the other hand, these codes are also focused in the specific phenomena regarding safety, i.e. analyzing parameters such as critical heat flux, pressure propagation and many others.

d. Computational fluid codes or similar: Computational Fluid Dynamics (CFD) simulation tools are in charge of solving the mass, energy and momentum balance equations of the fluid and/or species in a high level of detail. At the moment, CFD codes are used for multicomponent analysis in single-phase fluids, whilst the evaluation of two-phase fluids is still in development phase. Therefore, there are other codes that can model the thermal-hydraulics in pseudo-3D accounting two-phase flow.

e. Coupling of the different codes, coupled codes: the coupling of different codes is done to take advantage of the feedback phenomena in the physics that is present in the core behavior. This is done by designing a coupling scheme between codes of different physics. The most usual coupled codes are the combination of Neutron Kinetics and Thermal-Hydraulics, and the Thermal-Hydraulics with Fuel behavior codes. 
All these computational tools provide realistic results, in comparison with the conservative approach, that in certain cases the variable predicted can be close to the acceptance criteria (see Figure 2.5). For that reason, the predicted results must be complemented with the corresponding sensitive and uncertainty analysis. On the one hand, the sensitivity analysis sets the statistical correlation between the selected input variables assumed to influence on the uncertainty of the target output variable. Through this analysis it can be elucidated which input variables have more influence in the uncertainty of the target output variable. On the other hand, an uncertainty quantification is the way to account the uncertainty associated to relevant parameters, such as measures, initial and boundary conditions, plant data and so forth. The way of combining the input uncertainties through their probability density function is the way to account the overall uncertainty.

Therefore, two kinds of uncertainties must be described and treated separately. The first one is the epistemic uncertainty. This uncertainty derives from the lack of knowledge or the incomplete data. Parameters related to this type of uncertainty have a definite but not accurate known value. However, this type of uncertainty accounts also the simplifications assumed for modelling certain aspects. The epistemic uncertainty is defined with deterministic and also probabilistic models accounting the sensitivity analysis. Through these computational procedures it is possible to quantify the principal sources of uncertainty.

On the other hand, aleatory uncertainty wraps the random behavior of the system and its parts, as well as the related parameters of the plant operation. This type of uncertainty is evaluated from the probabilistic safety assessment in order to quantify the event occurrence of a system failure coming, for instance from the random failure of any safety function. Therefore, it is a way to quantify probabilistically the reliability of the system.

This manner to complement the predictions of the safety variable during the safety evaluation of the plant behavior is addressed in the BEPU approach. Compared to the different conservative approaches, the BEPU approach is applied to provide safety analysis with more realistic assumptions and conditions that allow a reduction of the safety margins within the guidelines of the Nuclear Regulatory (see section 2.5.2).

\subsection{Relevant steps of a BE simulation methodology.}

The nature of a NPP, as well as the reactor core, is a multi-scale nature. This feature is due to the fact of the heterogeneity of such system. This heterogeneity is related to different scales where different phenomena take place. The NPP is made of different components such as pipes, pumps, valves and the core. The core is made of fuel assemblies and each fuel assembly is made of fuel rods containing the fuel pellets inside the fuel cladding. 
Development and validation of a multi-scale and multi-physics methodology for the safety analysis of fast transients in Light Water Reactors

A local perturbation can affect the behavior of the reactor core and vice versa, therefore it is necessary to account every scale to evaluate properly the global effects of the core behavior in the corresponding macro, meso or micro-scale. In addition, it is necessary to account that many safety variables are evaluated locally, like the BE and also, different physical behavior must be evaluated at the corresponding scale. Moreover, the evaluation of such local phenomena would be imprecise or would result in an averaged value when not used the corresponding level of detail in the scale of analysis.

For instance, as an example, the safety variable Critical Heat Flux depends on the actual heat flux provided divided by the heat flux that would cause rod damage, according to the power being generated and the flow regime and heat removal capacity. Using a coarse channel-by-channel mesh for the core nodal distribution will provided an averaged value of the CHF. However, if the conditions of the transient affecting the channel are applied in a pin-by-pin model, it will be possible to track the critical fuel pin where the minimum $\mathrm{CHF}$ is located, and even the axial position.

Furthermore, it will yield in more accurate results if the applied physics for the critical channel designed in a pin-by-pin model is done by means of a pseudo-3D thermal-hydraulic code that accounts the two-phase flow with three fields, i.e. liquid, steam and droplets, than a code that is defined only in 1D for two-phase flow. Consequently, a multi-physics and multi-scale methodology will consider different scales and different physics, as Figure 3.1 shows, to take advantage of the computational power with a consensus with the required detail level and necessary physics.

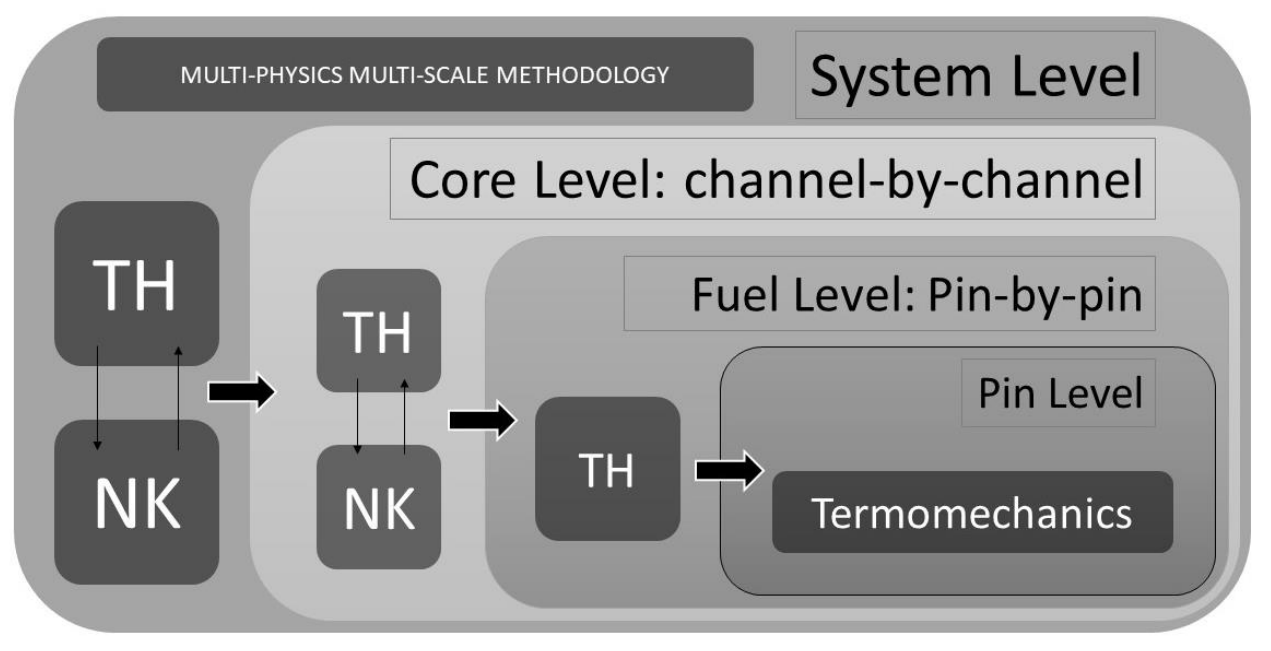

Figure 3.1 Diagram of the multi-scale feature of the proposed methodology. 
Moreover, the use of different physics includes the combination and coupling of the core physics when it applies. These means for example, that the interaction between the thermal-hydraulics and the neutron kinetics is accounted for system and core simulations, and that the interaction between thermal-hydraulics and thermo-mechanics is considered regarding the fuel behavior.

The methodology proposed in this thesis work applies the corresponding physics or coupled physics at the corresponding scale level in different steps, using the information generated in one step to simulate the transient in a higher level of detail with the corresponding physics.

This methodology uses the different state-of-the-art tools, which can be used for different purposes in the engineering department of the NPP and combines them adding value to their regular use by turning them into an efficient safety-oriented methodology for transient behavior in the NPP. Further subsections will explain the details of the proposed methodology.

The presented methodology was designed to be used whether step-by-step or modular wise. On the one hand, the step-by-step mode goes through the different steps needed for the analysis of the target safety variable.

First of all, it is necessary to identify the transient case to be evaluated and select which will be the safety variable to track along the different steps of the methodology.

1. The initial step is applied to generate the $3 \mathrm{D}$ cross-section libraries. The purpose is providing for the next step the Cross Section sets for each node of the 3D core physics code. The Cross-section libraries will provide different sets of cross sections for a range of State Variables, whether Temperature of Fuel, Density of Coolant, Control Rod Insertion and Boron Concentration. The effects of the historical values regarding the core burnup level also are accounted for the Temperature of Fuel, Density of Coolant and Control Rod insertion, as well as the exposure. For completing this procedure, it is necessary to use a Transport Lattice code. Usually, these codes are used for defining the fuel lattices and solving the transport equation for the depletion of the fuel lattice in a multi-group scheme. This is done in order to provide homogenized and few-group collapsed cross section data that usually is managed by a suite of Transport Code / Coupled thermal-hydraulics and neutron kinetics. Some examples of these suites are CASMO/SIMULATE (Pusa \& Isotalo, 2017) and HELIOS/PRESTO (Giust et al., 2010). The corresponding interface application manages the output of the transport lattice code and provides the cross-section data in the corresponding format to be read by the 3D Neutron Diffusion code. A code-to-code verification prior to proceed to the first step is advisable, in order to assure the correct data flow via the verified results. 
Development and validation of a multi-scale and multi-physics methodology for the safety analysis of fast transients in Light Water Reactors

2. The second step uses a TH system model of the plant and its main controllers, signal variables and components of the primary and turbine. The system model includes a vessel with the different components including the fuel channels. The fuel channels of the core can be lumped in order to save computational resources and giving the needed accuracy. The vessel of the system model is coupled to a channel-by-channel core modelled for a 3D nodal NK code solving the neutron diffusion equation in two groups. This NK core model is fed by the 3D Crosssection libraries from the previous step. Notice that the range of State Variables corresponds to the possible values of Temperature of Fuel and Density of Coolant that can be reached during the transient case.

3. The third step goes to the analysis of a TH core model designed channel-bychannel with a TH subchannel code. Once again, this TH core model is coupled to the 3D NK code with the corresponding Cross-section libraries. The previous step provides the boundary conditions of the core that will be simulated channelby-channel. This time, using this scale level is it possible to track the critical fuel channel according to the selected safety variable.

4. The fourth step uses the boundary conditions of the channel-by-channel coupled simulation. The conditions of the critical fuel channel are loaded in the corresponding TH model designed in a subchannel code pin-by-pin, including the water rods, the partial length rods that it may have or the guide tubes. This step in a detailed scale level is used to locate the critical fuel rod according to the local phenomena that the boundary conditions from the previous step.

5. Lastly, the boundary conditions of the critical fuel rod are loaded in a single rod model designed for a fuel behavior code. This step includes the detailed thermomechanics for the fuel pellet, gas gap and cladding of the fuel rod, therefore it is possible to account more phenomena at pin level providing the exact location in the rod of the critical axial node.

6. The results of the last step are complemented with an Uncertainty and Sensitivity Analysis. The user of the proposed methodology, as an expert must decide supported by the scientific literature, which parameters are of relevancy regarding the uncertainty of the target output variable. The uncertainty of the selected input parameters is introduced by generating a set of input samples where the input parameters are varied according to their probability density function. The result is a probability distribution of the output variable, in which a statistical criterion is applied in order to set the probability of the sample to be inside the defined Confidence Interval.

On the other hand, it is possible to use different steps as a stand-alone module, if the boundary conditions are provided. This can be applied to evaluate the fuel behavior of 
the fuel channel designed pin-by-pin, in order to elucidate possible event both transients and even in steady-state.

One important feature of this methodology is to account with a library of several input and complement data for modelling the target scenarios. First and foremost, it is important to have the availability of cross-section libraries for each burnup step of every fuel cycle from the NPP where this methodology is going to be applied. Therefore, as a parallel work to the application of the methodology, it is necessary that the cross-section libraries are generated and available. In that way, to apply the methodology, it will only be necessary to load the corresponding cross-section libraries.

Secondly, it is possible to have the different core configuration for the subchannel code, if the detailed subchannel fuel models are available. This means that on the one hand, it is necessary to have a full database of the different fuel assemblies modelled in detailed pin-by-pin scale that have been part of the core in the corresponding fuel cycles during the lifetime of the NPP. This database will be updated if necessary, for each new fuel cycle. Therefore, this is another parallel work to be undertaken in the application of the methodology. On the other hand, it is possible to generate the channel-by-channel fuel core model by lumping the geometric parameters of the fuel subchannel models and composing the core according to the fuel map. Figure 3.2 shows schematically how the different fuel assemblies are modelled pin-by-pin. The corresponding subchannel thermal-hydraulic parameters are averaged in a lumped fuel channel that is defined in the corresponding core channel-by-channel model. CTF-UPVIS code needs the wetted perimeter and flow area of the subchannel to undertake the cell calculations. By means of a MATLAB-based programmed application, the equivalent geometrical parameters are averaged to turn the pin-by-pin geometry of the fuel assembly model, to a lumped channel in the core model. 

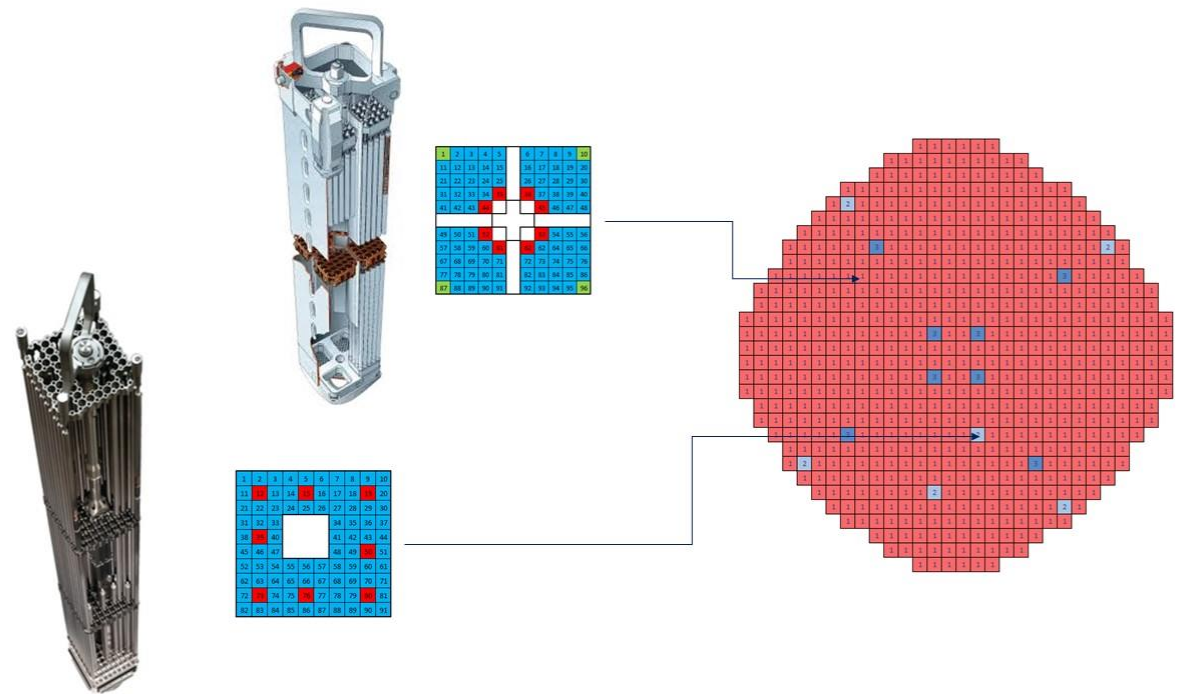

Figure 3.2 Diagram of the composition of the channel-by-channel core from the pin-by-pin fuel models.

The management of the data flow from one step to another, has been automatized by means of MATLAB ${ }^{\odot}$, Linux bash scripts and FORTRAN based applications. The purpose of this feature of the methodology is not only having an easy procedure to obtain the necessary safety evaluation but avoiding the user interference. The different interfaces in between the different steps of the proposed methodology are in charge of:

1. Tracking the critical component (fuel channel, fuel rod, etc.).

2. Retrieving the boundary conditions during such critical element during the simulated transient scenario.

Post-process the retrieved data and load the boundary conditions in the model of the next step with the corresponding output.

\subsection{Features of the methodology and the selected simulation tools.}

The different steps of the methodology have been described (see section 2.3). This subsection will go in detail of each step describing the specific procedure and its value addressed to the presented methodology.

\subsubsection{Generation of cross-section libraries.}


Diffusion codes are used in Safety Analysis as an approach to the Neutron Kinetics of the reactor core. Core simulators predict the neutron population behavior in the core and are usually coupled to thermal-hydraulic codes to account the feedback between the NK and TH in fast transients or during the fuel cycle calculation in a BE approach. It is of the state-of-the-art to provide these codes with Cross-Section Libraries that contain the cross-section sets for each core region defined in the simulation model. Most of the core simulators solve the diffusion equation in 2 energy groups. In addition, the geometrical characteristics and materials of the different fuel assemblies constituting the core are "blended" in the calculation nodes. Figure 3.3 and Figure 3.4 show the data flow diagram from the initial cross-section data to the final use in the core simulator code and a schematic representation respectively.

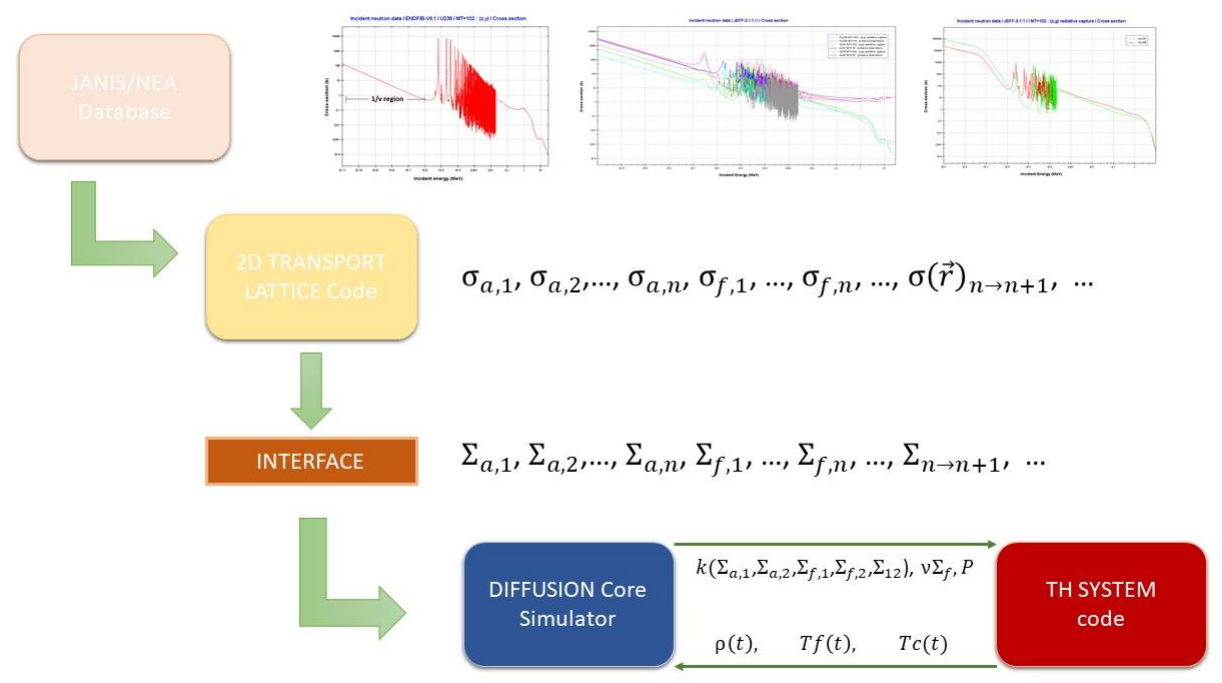

Figure 3.3 Data flow of the cross-section data.

According to Figure 3.3, transport lattice codes use the ENDF (Kahler et al., 2011) microscopic cross-section data in built-in libraries. These codes solve the transport equation in 2D detailed lattices working with several energy groups. One of the features of transport codes is that they are able to generate cross-section data libraries suitable for core simulator codes, which solve the Neutron Diffusion equation in averaged 3D cells.

The action of obtaining an equivalent approach of the cross-section from several energy groups to 2 groups is called collapsing. The action averaging the effect of cross-section weighted according to their geometrical design and material composition is called homogenization. Figure 3.4 shows schematically how the fuel assembly is modelled in 2D lattices with high level of detail (1). The information in regard of cross-section is collapsed and homogenized to be assigned to different fuel regions (2), that will be adapted 
Development and validation of a multi-scale and multi-physics methodology for the safety analysis of fast transients in Light Water Reactors

to the axial nodal distribution of the model. The different regions will form the 3D model for simulating the core. This simulation, as commented, can be coupled with a TH system code.
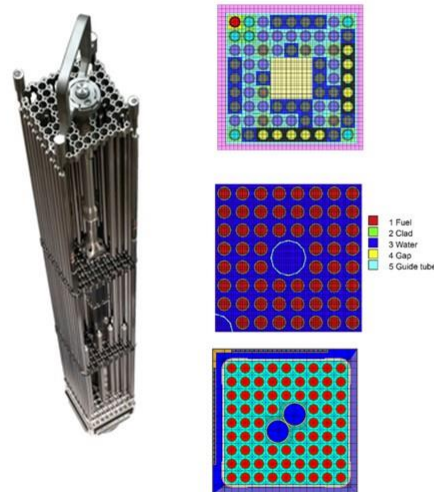

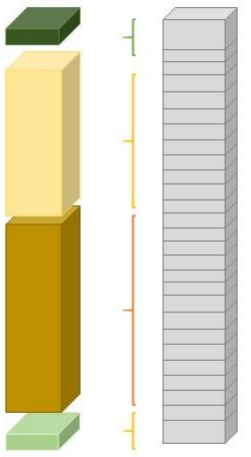

2)

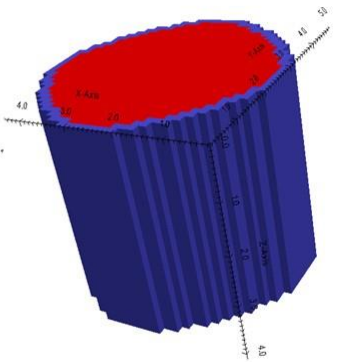

3)

Figure 3.4 Graphical representation of the calculation spaces.

A cross-section set must be understood as the set of the main Macroscopic Cross-Sections (Absorption, Fission, Scattering, Diffusion Coefficients and so forth) for each of the considered core regions. The core regions correspond to each 3D node or node groups that simulate a homogeneous composition of materials and geometry, in which the assigned cross-section set is valid. For instance, according to geometry, a Reactor Core can be modelled as a 3D nodal space where each fuel assembly design corresponds to a homogeneous core region. Moreover, a more detailed model would include regions for different homogeneous parts of the same fuel assembly type. On the other hand, fuel assemblies of the same design but with different fuel rod composition like Gd rods, can also constitute different core regions. This would be generating core regions depending on materials.

The cross-section sets of each defined core regions are provided for a range of TH States, Control Rod types and Burnup. TH States include different ranges of Fuel Temperature, Coolant Density and so on. The purpose of these ranges is to cover a spectrum of possible operation points in which the NK can be solve for each core region during a simulation. Moreover, the effect of a rod insertion in a calculation cell is retrieved by modifying the cross-section set of the region according to the composition of the corresponding control rod, which is stored in the library. Furthermore, the variation of the nuclear composition with the cycle exposure is accounted for each predefined burnup steps. Accounting the 
burnup allows to introduce the effect of the burnup in the cross-section data. For instance, the absorption cross-section of the homogenized fuel pellet, does not have the same value for fresh fuel than for a fuel used during 2 cycles. By accounting the burnup one can adapt the needed cross-section data to the burnup step of the simulation case. Finally, accounting the different burnup in the homogenized material is useful to account the so called historical effects in the cross-section. For example, the material of the fuel assembly will have a different fission cross-section if it has been burned at low coolant density, i.e. at the upper location of the fuel assembly, instead of at the bottom nodes. Top nodes with low density endure less fissions due to moderation effect than bottom nodes with low or no void fraction (high density of coolant). Therefore, the isotopic composition after a fuel cycle will be different among nodes that had more or less fissions. The result will be nodes with different cross-sections for fission. This reasoning can be extended to every cross-section type and its dependence on thermal-hydraulic variables, exposure (burnup) or control rod effect.

With Cross-Section Libraries, Core Simulators can solve the Diffusion equation for a wide range of steady-states, e.g. Beginning of Cycle (BOC), Cold Zero Power or different Control Rod configurations cases. Furthermore, Core Simulators allowing coupled transient calculations can use the cross-section sets corresponding to the varying thermal-hydraulic variables requested each calculation step of the simulation.

As explained, these Cross-Section Libraries provide a discrete range of values as a function of different TH and NK variables. Nevertheless, the calculation procedures usually use fine time step distributions for solving the TH and NK equations. This fact brings about the need to interpolate. Interpolating values of cross-section is a very sensible task due to the non-linear behavior of these variables. The work developed in this thesis has used different approaches that have shown interesting results, as summarized in Chapter 3. Two standard methods are used currently in regards of interpolating cross-section data, which in last term, defines the required code features in Neutron Diffusion simulations. Further development is given in this subsection.

The proposed methodology takes advantage of one initial step for generating 3D CrossSection Libraries. In this step, the sets of Macroscopic Cross-Sections for the different segments modelled in the core are generated in the corresponding format for the used 3D Neutron Diffusion code.

Using this multi-dimensional matrix of combinations between the selected variables, i.e. Temperature of Fuel, Density of Coolant, Control Rod insertion and so on, allows the 3D Neutron Diffusion code to use the Cross Section data that fits with the thermal-hydraulic state of the node.

This formatted information must account the needs of the 3D neutron diffusion code and the format of the output files of the corresponding lattice code that is being used as source for generating the homogenized and collapsed in two group cross section data. For that purpose, different interfaces have been tested in the methodology presented in this thesis. 
Development and validation of a multi-scale and multi-physics methodology for the safety analysis of fast transients in Light Water Reactors

The use of 3D Cross-Section Libraries comes from the necessity of modelling the coupled behavior of the core regarding the TH and the NK. It is known that the transient behavior of the core can have very strong asymmetries depending on the postulated scenario in the Safety Analysis. For that reason, it is a BE tool to account this behavior by acknowledging the 3D simulation of the effect in the cross sections and the thermalhydraulics. With the use of other approaches, as the 1D Neutron Kinetics or Point Kinetics, the core would be simulated as a uniform element, and the local effects caused by the asymmetric behavior would be neglected. With a 3D NK distribution, it is possible to account the feedback in transient cases such as Control Rod Accidents. In such events, a control rod is rapidly withdrawn causing a local reactivity peak. For these cases, the use of Cross-Section Libraries in coupled 3D TH/NK give a BE approach.

The work developed for this thesis has accounted two different ways to obtain CrossSection Libraries. Both methods are extended and used in the SOA of the BE simulations. These two methods are the use of Tabulated Cross-Section Libraries and the use of functional derivatives. The former uses the raw values of the corresponding macroscopic cross sections whilst the latter uses only a reference set of Cross-Sections where the effect of the change of TH variables is lineally added to the reference value.

There are currently two extended methods to generate Cross-Section Libraries that cover the necessary range of thermal-hydraulic variables governing the behavior of the core during the simulated transient. These two methods are the parametrized libraries of crosssection data and the multidimensional tabulated ones (Ferroukhi et al., 2009).

The conventional form of generating cross-section libraries for diffusion codes is the multidimensional tabulated scheme. The user defines a mesh that covers the range of state variables, fuel temperature, coolant density and control rod insertion. Then, the corresponding cross-section data is calculated for each mesh point. The Diffusion code must use the corresponding algorithm to compute the necessary cross section data during the simulation by means of interpolating between the corresponding mesh points. This method has the advantage of not using functional forms for approximating the cross section dependencies, sparing the effort of defining the corresponding accurate polynomials. On the other hand, the interpolation process has attached certain level of uncertainty that is reduced increasing the number of mesh points. Therefore, the size of the libraries can tend to be critical for the data storage and handling.

On the other hand, parametrized libraries save significant storage capacity by including models of the cross sections dependencies on the state variables. The main drawback is that constructing such models requires of long polynomials that may incur in loss of accuracy in certain parameter ranges.

Chapter 4 will present a summary of the work developed by in this thesis in regard of the use of Cross-Section Libraries. The chapter shows a verification process for comparing one State-of-the-Art methodology for generating Cross-Section Libraries, the so- 
called SIMTAB methodology and another one, currently under development, with additional and improved features: GenPMAXS methodology. 
Development and validation of a multi-scale and multi-physics methodology for the safety analysis of fast transients in Light Water Reactors

\subsubsection{The NK diffusion codes.}

The $3 \mathrm{D}$ cross section data is generated to be used in a 3D neutron diffusion code for core simulations. This simulation tool gives the chance of obtaining a compromise between detailed calculations and efficient computational time. The compromise is obtained by means of solving the Neutron Kinetics transport with the approach of the Diffusion Theory.

The core of a LWR is defined using a 3D mesh of nodes, usually cubic cells of $15.24 \mathrm{~cm}$ side length, where each node is related to a part of a fuel assembly or reflector. In this way, the heterogeneity of the core can be accounted, which is a key factor for the simulation of asymmetric transients with where the neutron flux distribution in $3 \mathrm{D}$ must be predicted.

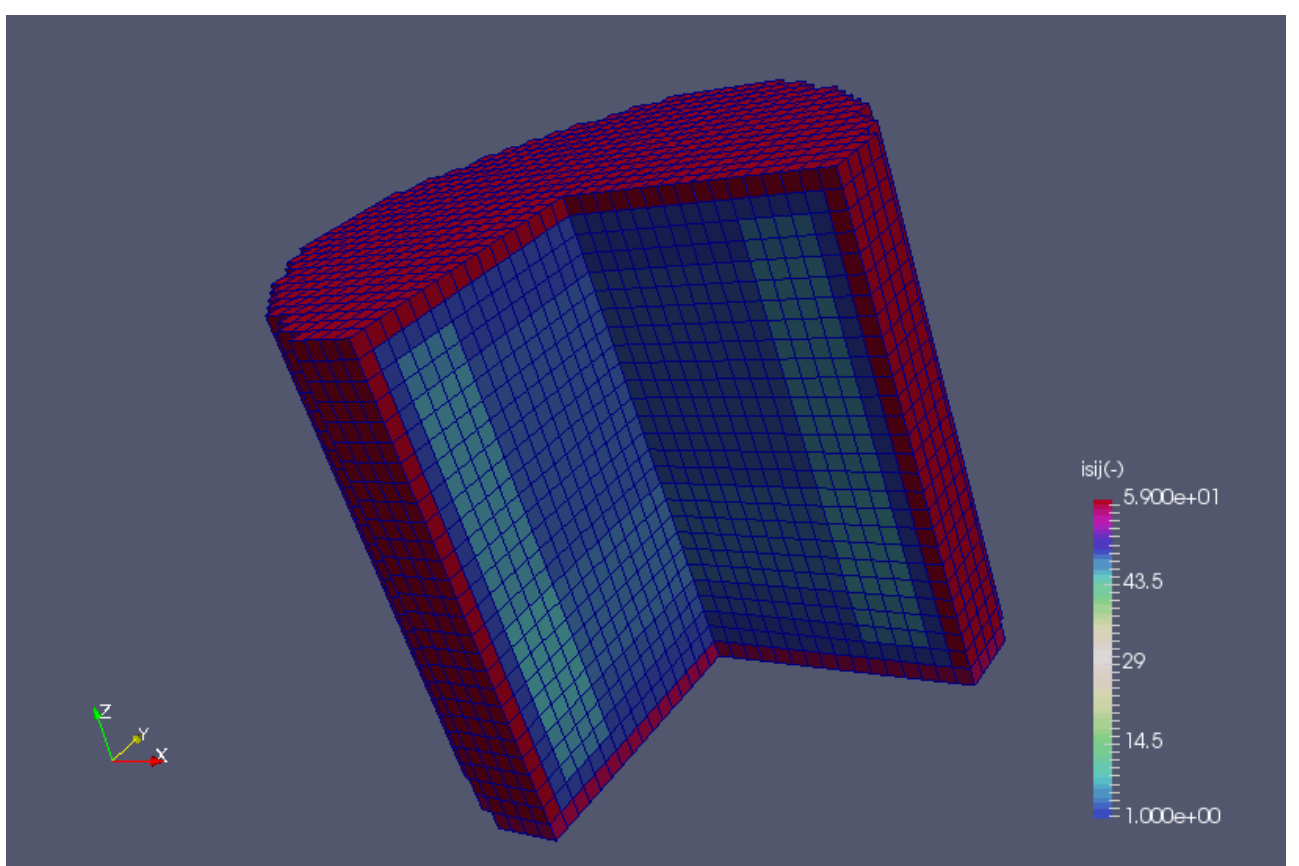

Figure 3.5 Nodal distribution of a BWR for a Neutron Diffusion 3D code.

Therefore, the use of the 3D core model with the corresponding Cross-section libraries allows a more accurate prediction of the neutron flux for further uses in deterministic safety analysis. The methodology presented in this thesis uses the code PARCS using two groups of energy and 6 groups of delayed neutrons.

\subsubsection{Simulation of system models: System codes and NK coupling.}

Several transients need accounting the behavior of different components of a NPP for an enhanced safety analysis. The role of components of safety systems and subsystems must 
be evaluated for a complete simulation of certain events. For example, during a Turbine Trip, the modelling of the behavior of valve closures or pipe pressure gives a more accurate prediction of the overall response of the core. Therefore, in deterministic safety analysis it is normal to use the so-called system codes. It is important to account that besides in the Reactor Core, there is no need to account the calculation of the neutron flux but only the thermal-hydraulic behavior. Because of these, system codes are meant to solve the conservation equations of mass, momentum and energy of the coolant fluid operating in the system. Moreover, there are transient cases to be evaluated that do not necessary need the evaluation of the neutron kinetics, such as Loss of Coolant Accidents. Nevertheless, there are other transient cases, like the instability events in BWRs, that need to account the feedback between both physics, i.e. neutron kinetics and thermalhydraulics, in order to give a more realistic calculation. The feature will be detailed in the following subsection.

However, the build-up engineering experience in the nuclear safety analysis reveal that system codes modelling components in one or two dimensions, although some of them such as the reactor pressure vessel is convenient to be modelled in $3 \mathrm{D}$, are enough to bring about satisfactory results for the evaluation of transient cases. For this point of the simulation methodology of the current thesis work, the overall behavior of the nuclear power plant is intended to be modelled with a system thermal-hydraulic core that gives the preliminary overall response of the core and relevant components during the target transient case to be simulated.

Different qualified codes have been used for this purpose along the history of deterministic safety analysis. For the work presented here, the system is modelled with TRACE code. Figure 3.6 shows the scheme of a system input deck of a BWR model, printed with SNAP tool. 


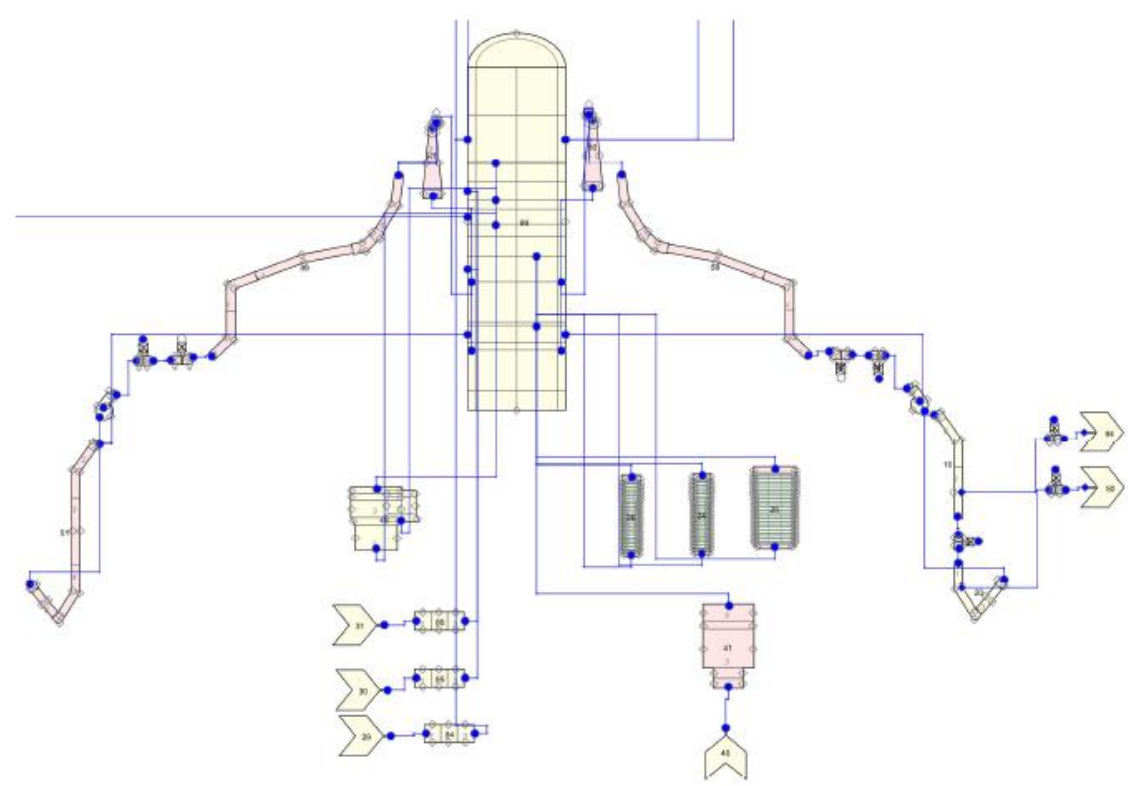

Figure 3.6 System input deck of the RPV and internal components, visualized with SNAP.

\subsubsection{Coupled TH/NK subchannel core simulation.}

As it has been commented, certain transient cases include a strong feedback between the evolution of the neutron kinetics and the thermal-hydraulics. The BE approach in Deterministic Safety Analysis was achieved by accounting not only the known physics separate models, but also the feedback mechanisms between them. To be more specific, in LWRs, since the water acts as coolant and moderator, there is a relevant feedback behavior between the properties of the coolant and its capacity to moderate or not neutrons. Therefore, not accounting this fact, could yield the necessity of using more conservative assumptions to compensate the not modeled physic behavior.

The methodology presented here accounts this physical effect by means of using coupled models of the reactor and plant. These models use a coupled scheme between a 3D neutron diffusion code and a thermal-hydraulic code. It is important for the nodal distribution to be coherent, therefore, special effort must be put in mapping of both models, so the information during the calculation can be properly exchanged.

The user must introduce the equivalence between the NK radial and axial power distribution with the power generation components of the TH system code. For this purpose, the user will decide the accuracy of the power modelling by deciding how many fuel channels of the 3D NK code are lumped in the heat structures of the system code. 


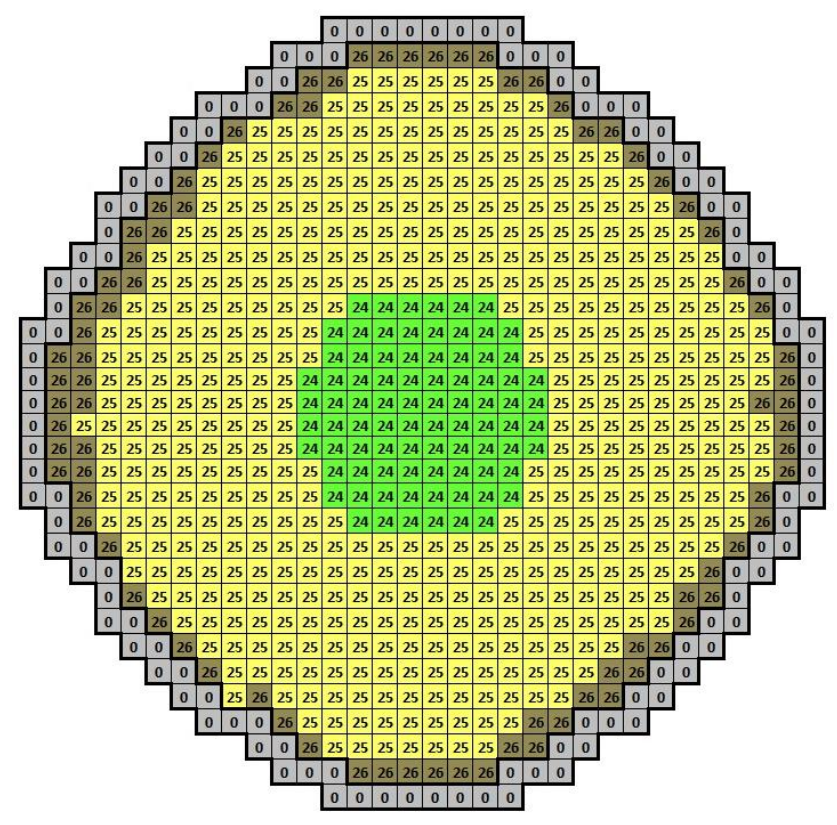

Figure 3.7 Mapping of TH system code and 3D NK code.

The strategy of this decision must account that coarser meshes use more averaged feedback information but finer meshes require of more computational power. Figure 3.7 shows the mapping of a channel-by-channel radial distribution of a 3D NK code that is coupled to three different averaged heat structures of a system TH code.

For the coupling scheme, both codes exchange the corresponding information with a coherent distribution of the time and space of the problem. Therefore, at each time step and each node, the TH code uses the power developed via Nuclear Fission to predict the evolution of the thermal-hydraulic variables such as fuel temperature or coolant density. This prediction is sent back to the neutron diffusion code so the corresponding cross sections of the node can be obtained, using the Cross-section libraries. The diffusion equation is solved, and the power developed at the node due to the neutron flux causing fissions is given back to the thermal-hydraulic code.

This calculation step gives an overall and realistic behavior of the core during the postulated transient case. In this way, it is possible to evaluate the evolution of different variables, regarding thermal-hydraulics or neutron kinetics. The purpose of the proposed methodology is to obtain with this calculation the boundary conditions of the core in a $\mathrm{BE}$ approach. This information will be used in further steps for more scale detailed simulations.

\subsubsection{TH subchannel fuel assembly simulation.}


Development and validation of a multi-scale and multi-physics methodology for the safety analysis of fast transients in Light Water Reactors

The analysis in the subchannel scale allows the evaluation of safety variables accounting more local phenomena. The scale of this approach can be used to model whether fuel channels in the core, or the behavior of the coolant between the fuel pins of an assembly.

These simulation tools use more detailed 3D geometry models as well as more complex physical models. The presented methodology is based on the use of the subchannel code CTF-UPVIS, from the COBRA-TF family codes.

The use of a channel-by-channel core model allows to locate the critical fuel channel during a core simulation. In the methodology, this step is done loading the boundary conditions of the Coupled TH/NK system model in a detailed channel-by-channel core model. The proposed methodology is designed to retrieve the boundary conditions of the core that have been simulated in the previous step and load them in the corresponding channel-by-channel core model applying also a coupled scheme of Thermal-Hydraulics and Neutron Kinetics. Therefore, it is possible to introduce the evolution of the system behavior in a more detailed scale, without assuming the computational cost of simulating the whole system with such fine mesh.

The process is made automatically by means of programed applications in order to avoid the interference of the user. Afterwards, the transient is again simulated but this time in a finer mesh. The result is the availability of tracking the critical fuel pin according to the assigned safety criterion. For example, if a Reactivity Insertion Accident is being simulated, the methodology will be focused in detecting the maximum BE as safety variable, hence, the fuel channel showing the highest value during the transient will be located.

However, the methodology developed in this thesis does not limit to the application of such codes in a channel-by-channel core analysis. Once the critical fuel channel is located, the specific boundary conditions are retrieved and loaded in the corresponding pin-by-pin fuel assembly model. This step gives a more detailed scale analysis by means of accounting specific parts of the fuel assembly to be modelled, such as the partial length fuel rods or the Water Rods. Moreover, the simulation of this step will be used for locating the critical fuel pin, as it has been done for the critical fuel channel in the previous step.

Notice that the previous step only allows to retrieve information about the thermal-hydraulics of the transient regarding safety variables like the CPR. Further effect on the behavior of the fuel cladding, or even the fuel pellet is not considered, or considered with simpler and conservative models. Nevertheless, it is possible to analyze in a more detailed scale by going a step forward in the proposed methodology. The prediction of the subchannel thermal-hydraulic conditions in this step can be used as detailed boundary conditions in a fuel pin model of a fuel behavior code. Usually, these thermomechanic codes are focused in fuel material behavior, and led the thermal-hydraulic conditions to 
more conservative assumptions or built-in models. The combination of the thermal-hydraulic subchannel analysis with the thermomechanic analysis leads to the next step of the proposed methodology.

\subsubsection{Fuel behavior analysis of the critical fuel pin.}

As the Nuclear Regulation Authorities specify, for certain postulated events, when necessary, it is required to evaluate the behavior of the fuel rod in order to assure that no fuel damage will take place, or at least the fuel damage is kept in the corresponding safety margins. These requirements make necessary the use of codes evaluating the performance of the fuel rod by means of applying the physics governing the thermo-mechanical phenomena inside the fuel cladding.

This methodology uses specifically the fuel behavior code suite FRAPCON/FRAPTRAN. In this way, it is possible to analyze the consequences of the heat generation and transfer through the rod and the evolution of this fact when the thermal-hydraulic conditions change during the simulated transient.

A deteriorated heat transfer condition in the cladding leads to internal stress and chemical reactions derived from fuel temperature increase. Therefore, it is necessary that the local thermal-hydraulic conditions predicted in the previous step of the methodology are implemented in the corresponding fuel pin model. This last step will allow predicting variables such as the oxidation, generation of hydrogen or cladding deformation.

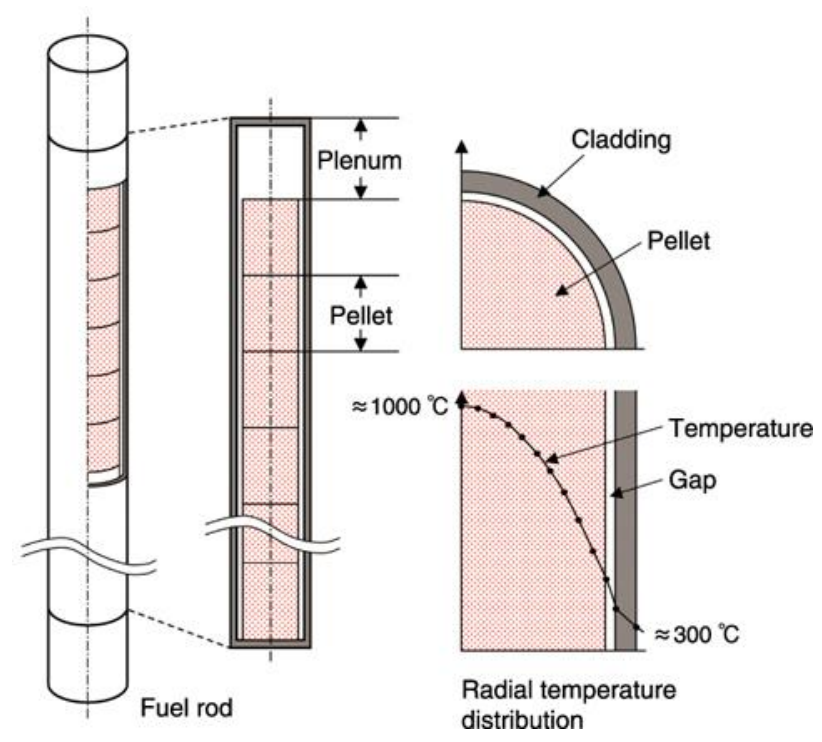

Figure 3.8 Heat Transfer scheme of the fuel rod.

Moreover, the use of FRAPCON/FRAPTRAN allows to set a more realistic approach of the distribution of the fuel gap heat transfer capacity. This fact means a more realistic 
Development and validation of a multi-scale and multi-physics methodology for the safety analysis of fast transients in Light Water Reactors

analysis regarding the previous step, where this factor has to be assumed constant for the whole fuel rod.

The heat transfer analysis and the consequent thermo-mechanics are provided by this step, adding to the presented methodology a complete multi-physics and multi-scale feature.

\subsubsection{The need of the $U \& S$ analysis.}

As the requirements of the nuclear authorities set, for a BE approach it is necessary to proof that the limits assigned to the corresponding safety variable are not trespassed. This is carried out using an uncertainty quantification of the predicted safety variable.

These safety guidelines consider enough to meet the 95/95 criterion when predicting a safety variable. This means that for the $95 \%$ of the cases, the safety variable has to be predicted with in a $95 \%$ of the Confidence Interval of the mean value, i.e. that the predicted value must not be deviated from the man in 2 times the calculated standard deviation.

In addition, using a BE approach in the prediction of safety variables can incur in a reduction of the safety margin. Conservative approaches give a wide margin since their predictions are based on assumptions that usually consider the most limiting case due to physical model limitations or coarse mesh definitions. Conversely, using the realistic values in the BE codes predicts closer values to the real margins (see Figure 2.5). For that reason, it is necessary to account with statistical techniques in the evaluation model in order to assure that the upper or lower margin of the prediction of the safety variable does not trespass the assigned limits.

This process includes the simulation of a sample, the size of which has to be determined using the corresponding statistical techniques. The approach used in the methodology of the thesis is based on the Wilks method, as a manner to obtain a reasonable size of the sample. Nevertheless, other methods for the quantification of the uncertainties can be adopted using the corresponding statistical codes. The proposed methodology uses the DAKOTA [53] toolkit for sampling the simulation case and afterwards post-process the results retrieving statistical parameters such as the mean value or the standard deviation. The results are used for defining the Confidence Interval according to the proposed criterion.

Moreover, the use of DAKOTA toolkit provides with the option of evaluating the sensitivity of the target output variable. The correlation of the uncertainty of the input variables to be sampled and the uncertainty of the output variable reveals which input variables are more relevant for the prediction of the output variable. DAKOTA toolkit allows the use of different correlation coefficient. However, the present methodology uses the Partial Rank Correlation Coefficients (PRCC) considering the non-linearity between the output and input variables existing in the thermal-hydraulic and neutron kinetics analytical solutions. 
The Sensitivity Analysis is a feature of the proposed methodology that allows performing an evaluation of the correlation between the analyzed output variable, and the uncertainty of a selected set of input variables. For instance, when analyzing the input parameters affecting the uncertainty of the prediction of the void fraction. The prediction of void fraction in a thermal-hydraulic code is usually based in closure equations that are based as well in experimental data. These data uses coefficients that are adjusted to reduce the error of the modelling of the experimental results. A sensitivity analysis of such coefficients can be useful to reveal how a small variation in the coefficient as input can enhance the prediction of the void fraction. Therefore, the proposed methodology has the added value of being a tool for analyzing and enhancing physical models in simulation tools of the nuclear industry.

\subsection{Examples that summarize the application of the methodology to a real case.}

This subsection will provide a brief description of the application of the different steps of the proposed methodology with two hypothetical examples: one corresponding to the step-by-step simulation, and the other one of the specific modular application of one of the steps.

EXAMPLE 1.- SIMULATION OF A LOCA.

A LOCA scenario compromises the safety operation of a NPP (USNRC, 2007). The licensing of NPPs requires reporting the Safety Assessment of such transient cases. For that purpose, the methodology would be applied step-by-step till obtaining the final results of the target output variable selected as figure of merit. For this kind of transients, the integrity of the fuel cladding must be assured, therefore, it is necessary to prove that the temperature of the fuel cladding will not pass the safety boundary according to the corresponding Regulation Guide provided by the Nuclear Authority. The application of the methodology goes as follows:

1) The transient case is simulated at plant level to obtain the overall behavior of the core, accounting the feedback of the Neutron Kinetics and Thermal-hydraulics. A plant model is used for both coupled state-of-the-art tools, using the results of the cross section data from the previous step.

2) The evolution of the core resulting from the simulation of step 2 is used as boundary conditions for a channel-by-channel thermal-hydraulic core model coupled with the available neutron kinetics code. Moreover, this step will use the Cross Section Data from step 1. The results reveal which is the critical channel according to the selected safety criterion. For this kind of transients, the methodology will search in the results of this step, which is the fuel channel with the maximum BE value. The evolution of the channel variables is used as input for the next step. 
Development and validation of a multi-scale and multi-physics methodology for the safety analysis of fast transients in Light Water Reactors

3) In this step, the results of the channel transient simulation are loaded in a pinby-pin fuel assembly model for a more detailed thermal-hydraulic simulation. The methodology detects which type of fuel assembly model is to be loaded from the fuel assembly library defined for the core. The results of this step are used by the methodology to, according to the selected safety criterion, track the critical fuel pin.

4) The conditions retrieved at pin level from the previous step are loaded in a fuel pin model to be simulated in this new step. This step provides the thermo-mechanics analysis that can be use if necessary to the thermal-hydraulic analysis at pin level. For certain analysis where the integrity of the fuel cladding is needed to by analyze, this is a necessary step.

5) The results obtained at the last step of the application of the methodology are complemented with the corresponding Uncertainty and Sensitivity analysis.

6) Uncertainty quantification methods are available today, and several applications have been and will be performed in reactor safety research as well as in licensing. Experience of applications shows that the difference between predicted upper bound or $95^{\text {th }}$ percentile and $95 \%$ confidence level PCT to a calculation using nominal "best estimate" input values and default values for the computer code options and input data for models is about $200 \mathrm{~K}$ for a typical large break loss of coolant accident. These relatively large values are due to the numerous models and correlations that are incorporated into a thermal-hydraulic code, and to the uncertainties associated with those individual models.

\section{EXAMPLE 2.- SPECIFIC ANALYSIS OF THE BEHAVIOR OF A FUEL ASSEMBLY.}

A specific single step can be used as requirement of the engineering analysis of the operation of the NPP. The methodology will provide the corresponding input model and the conditions will be loaded for the different cases needed to be analyzed, generating a set of the output variables. The resulting variables retrieved from the scenarios to be simulated can be complemented with further steps or with the Uncertainty and Sensitivity analysis.

An example showing the use of specific steps of the methodology is as follows. When a new fuel assembly is introduced in the reactor core, the operator must fulfil the requirements of the Nuclear Regulator by showing that the insertion of a new fuel assembly in the core will not compromise the safety of the power plant for the different core operation modes, i.e. normal operation, shut down, AOOs, DBAs and so forth. This example considers the fuel behavior analysis of a specific fuel assembly in a LOCA event. In this case, the Technical Support of the NPP can perform a preliminary scope analysis by using the following steps: 
1) The Acceptance Criteria must be checked to obtain the safety variables to be analyzed. In case of a LOCA scenario, and according to the USNRC, the safety variables to be analyzed will be the PCT and if applies, the Cladding Oxidation, the generation of hydrogen, and the rod deformation to assure the fuel coolability.

2) The boundary conditions must be assumed according to the desired conservatism of the Analysis. A first approach can include a conservative scope analysis. Therefore, the boundary conditions will be assumed using the uncertainty of the input variables for the limiting case. This data can be checked in the corresponding regulation document.

3) The selection of conservative conditions for the analysis allows avoiding the U\&S analysis of the results. Hence, it is only necessary to perform the fuel behavior analysis of the critical fuel pin.

4) The corresponding input file will be defined using the available application for generating fuel pin models for the available Fuel Behavior code. For this purpose, it is necessary to gather the technical specifications if possible, or use information from the data base for input designs in the NPP.

5) The simulation of the case at this scale will provide the evolution of safety variables. This process can be automatized using an adhoc programmed application that retrieves the information from the output.

It can be summarized that the methodology is designed to be adapted to the specific case of the Nuclear Power Plant. For that purpose, each of the modular steps are to be adapted to the corresponding state-of-the-art tools available for the user.

The main feature of the methodology is the BE capability achieved with the selection of the computational tools and the possibility to apply these tools to the required scale. The $\mathrm{BE}$ results are complemented by an uncertainty and sensitivity analysis. This feature of the methodology can be done in two ways. On the one hand, the user will supply the uncertainty of the inputs variables in a specific step of the methodology analyzing the corresponding results for the selected output variable. On the other hand, the methodology will be able to evaluate the uncertainty from the initial step (the generation of crosssection libraries) and analyze the propagation of the uncertainty through the different steps of the methodology till arrive to the final results of the target output variable. 


\section{Chapter 4}

\section{Validation and Verification of codes and methodologies for two application cases}

The use of simulation tools and methodologies must meet the requirements of the $\mathrm{Nu}$ clear Regulator to be acknowledged as qualified simulation tools. The procedures for earning the qualification span from certifying the quality of the code programming and documentation to the third party review of the source code and implemented methods, and the validation of the results against real data, whether experimental or coming from plant operation records. The qualification procedure is a detailed project undertaken by specialized and qualified professionals in constant feedback with the corresponding institutions until the final product is released. This engineering and design procedure includes the development of methodologies for safety analysis, and as showed with CSAU and CASL projects in Chapter 1, it can be assumed that validating a methodology is a living project that usually becomes updated.

The scope of the qualification of a safety analysis methodology is beyond the results presented in this thesis work. Nevertheless, the development of this research work has yielded several validation and verification exercises that can contribute to further work in the framework of the development of simulation tools. Since the results obtained have been part of research projects in collaboration with different NPP and have been also published in different congresses, this chapter will summary the results as a parallel work to the validation and design of the proposed multi-scale and multi-physics methodology.

This chapter will introduce a summary of the international guidelines and different regulations for code and methodologies qualification for safety analysis in the first subsection. Subsection 2 will present the procedure of a code-to-code verification of the development of the GenPMAXS methodology for the generation of homogenized and 2-group collapsed cross section libraries. Finally, subsection 3 will show the validation of the 
Chapter 4. Validation and Verification of Codes and Methodologies for two Application cases

void fraction correlation in the subchannel thermal-hydraulic code CTF-UPVIS against the PSBT benchmark of the OECD/NEA with an uncertainty and sensitivity analysis.

\subsection{Code and Methodology qualification for safety analysis.}

Simulation tools are made for the assessment of Nuclear Safety in different stages:

a) Core design.

b) Core management.

c) Quantification of Conservative Analysis Margins.

d) Design of accident management procedures.

e) Analysis of operational events.

f) Safety Assessment of accident scenarios.

g) Licensing Objectives.

To that matter, it is necessary to grant their capability for representing and predicting the reality of the NPP behavior. A simulation tool includes features from different knowledge areas that interact one each other. These areas include physical models and numerical methods to solve these models, but also programming these solution schemes and modelling the analysis case. For this reason, International Standards and Nuclear Regulators require the assessment of the quality in the procedure of developing a simulation tool (Odar, 2000).

The process of qualifying a simulation tool includes code developing, improvement, and assessment. The so-called verification process consists of evaluating that the numerical methods are coherent for solving the core physics and models, as well as the coherence in the programmed source code.

The code assessment is done by sharing the code to independent users that will undertake different simulation exercises in order to reveal potential limitations and possible errors. For this purpose, there is available experimental data that is used to compare the results predicted by the code against real data. This allows the user to quantify the uncertainty of the code in regards of its capability to reproduce reality. For instance, COBRA-TF developers have gather in a user's group different experts and institutions that review, test and develop this simulation tool and put experience in common in a yearly international meeting (CTF User's Group, 2018).

The experimental data is of relevant importance, and several efforts are made by international institutions to gather, organize and give quality assurance of these data. From a general point of view, there are two types of experimental data, realized for different purposes. On the one hand, Integral Test Facilities (ITFs) such as ATLAS (KAERI, 2009) or PKL (Framatome, 2019), are scaled facilities to reproduce the behavior of a NPP. On the other hand, Separated Effect Test Facilities (SETFs) are facilities which are meant to reproduce specific phenomena of the core or different components in a NPP 
Development and validation of a multi-scale and multi-physics methodology for the safety analysis of fast transients in Light Water Reactors

and there are several facilities around the world. On a final stage, the developed simulation tool is proven in NPPs and the margins in calculation errors against real plant data are quantified with a suitable Uncertainty Analysis.

To sum up, the comparison of the results predicted by a simulation tool with a qualified reference is a necessary step for qualification. This step can be done in different parts and using different references. Further subsections of this chapter intent to give proof of this fact, for some of the codes used in the presented methodology.

\subsection{Methodology for the generation of Homogenized 2-Group Cross Sec- tion Libraries.}

In general terms, methodologies for generating accurate solutions for providing CrossSection libraries are of significant relevancy. Tabulated Cross-Section Libraries have been used for the work developed in this thesis. Two different methods have been used for generating such Cross-section libraries, one uses raw cross section data for each mesh point of the predefined thermal-hydraulic and state variables, that is the SIMTAB method, and the other one uses a reference cross section data and the corresponding derivatives for the rest of the branch points, namely GenPMAXS (Ward et al., 2016) method. For this purpose, the corresponding version of the Neutron Diffusion code must accept the format and algorithms of both methods. This work has been developed for PARCS code in the version 3.2.

The work developed in this thesis with the aim of setting a reliable method for generating cross-section libraries has derived in the code-to-code verification of both methods, showing the status, advantages and disadvantages and future work regarding this issue.

\subsubsection{SIMTAB methodology.}

SIMTAB method shows a conventional and simple way to generate cross section data libraries for the specific burnup step of the fuel cycle where the target simulation is located. The historical data is included directly in cross sections of the generated libraries named nemtab and nemtabr. This method takes the cross section data from the transport code CASMO, and uses online SIMULATE for compiling the cross-section libraries. Therefore, there are two drawbacks being the dependency of an external auxiliary code and the need of calculating the set of cross section data each time a scenario is postulated. The historical effects on the final cross-section data are calculated by CASMO and provided to SIMULATE through the interface CSMLINK. This code system has been developed by Studsvik Scanpower (Sweden) and needs for owner licensing for its execution, which is an additional drawback if it is not possible to own a license or collaborate with a code owner.

On the other hand, SIMTAB (Barrachina et al., 2011) is a well and reliable validated method. Its robust source code and the experience facilitates the use and helps to track possible programming errors when adapting the SIMTAB methodology to new and more 
Chapter 4. Validation and Verification of Codes and Methodologies for two Application cases

detailed core designs. Even though depending on an external code can be a disadvantage, taking advance of a developed and commercial code gives reliability to the results.

However, certain limitations have been observed when SIMTAB has been applied in this work. SIMTAB cannot acknowledge more than one control rod composition. This means that the library corresponding to the controlled cross section data, nemtabr, only accounts one type of control rod effect. This is not a problem for simplified core follow models that only account one control rod composition and modify the effect of the handle by means of the Gray Factor (Ferroukhi et al., 2009). Nevertheless, in the cases where the methodology of this thesis work has been applied, a more complex core model has revealed that more than one control rod composition must be accounted for $\mathrm{BE}$ results.

\subsubsection{Developed work on GenPMAXS methodology.}

At this point, an alternative method was used for the development of this thesis work. The GenPMAXS method shows the capability of accounting the historical effects of burnup, fuel temperature, control rod composition and coolant density, this means that the PMAXS libraries only have to be computed once, or updated each fuel cycle. Moreover, GenPMAXS method includes the capability of modelling more than one control rod composition, which makes it more suitable for representing complex core models. Another advantage is that GenPMAXS retrieves information directly from the lattice code. The case used for the application cases in this work retrieve the data from CASMO, but GenPMAXS can retrieve cross section data for a wide range of lattice codes.

Nonetheless, GenPMAXS is still a method under development, and the use of it reveals limitations that have been corrected or enhanced in the recent versions. For the specific case applied in this work, CASMO branch off procedure had to be reviewed and replaced. The branch off distribution is how the lattice code generates the range of different historical and instantaneous states that cover the range of the thermal-hydraulic behavior of the core. Each branch is a combination of thermal-hydraulic variables. These different combinations of state variables must be done in a specific way to fit the requirements of GenPMAXS. These requirements basically force avoiding the change of more than one state variable from one branch to another.

Figure 4.1 and Figure 4.2 shows graphically the differences between a possible branchoff in CASMO/SIMULATE and how must be structured the State Variables for a correct reading of GenPMAXS. 
Development and validation of a multi-scale and multi-physics methodology for the safety analysis of fast transients in Light Water Reactors

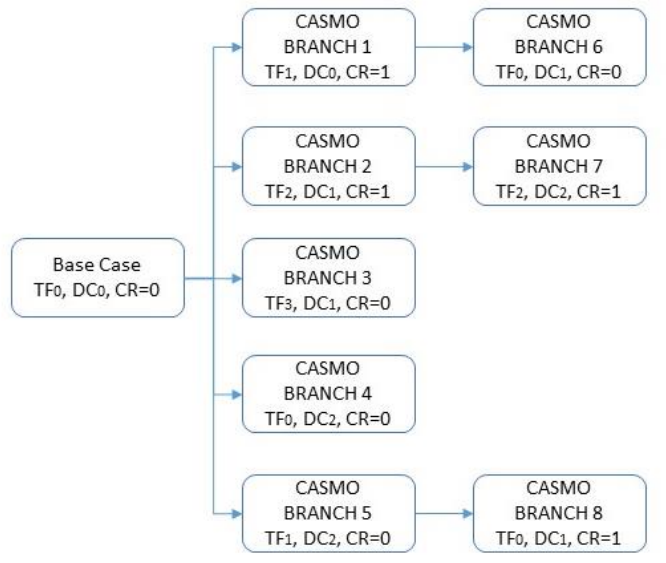

Figure 4.1 Approach of the built-in Branch Structure in CASMO.

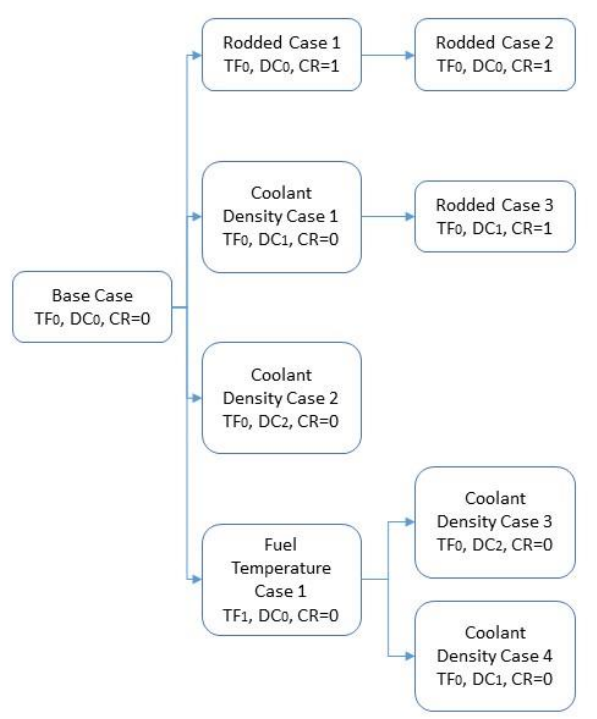

Figure 4.2 Adapted Branch Structure needed for GenPMAXS when reading CASMO output files. 
GenPMAXS considers a case base of raw cross-section data and the rest of branches store the partial derivatives on the different branch state variables, that change from one branch to another. The approach of GenPMAXS for the cross-section data treatment is implemented in PARCS code. Therefore, the disposed data must be coherent.

A simplified example would be retrieving from the PMAXS library the cross-section data set for a case of lower coolant density compared to the base case. First of all, we assume that the cross-section data defined for the base case is stored in the PMAXS library for the variables TF1, DC1 and NoCr, and the target state corresponds to a decrease of coolant density defined by DC2. The PMAXS library provides the partial derivatives of the cross-section for changing from the base case to the target case:

$$
\frac{\partial \Sigma_{f 1}}{\partial D C_{2}}, \frac{\partial \Sigma_{a 1}}{\partial D C_{2}}, \frac{\partial \Sigma_{12}}{\partial D C_{2}}, \ldots
$$

The cross-section data set of the target state is calculated using this group of derivatives and the incremental/detrimental of the variable that changes from one state to another. Considering the fission cross-section:

$$
\Sigma_{f 1}^{\prime}=\Delta D C \cdot \frac{\partial \Sigma_{f 1}}{\partial D C_{2}}
$$

Equation 4.2

The corresponding cross-section for this state $\Sigma_{f 1}^{\prime}$ is calculated according Equation 4.2. This would be a very simpler example. For more realistic and common calculations, we can think in interpolating derivatives when the target case locates the variables between two states of the library. Moreover, we have to consider that PMAXS libraries can depend on several state variables, so in the most complicated cases we would have to consider multiple interpolation schemes.

\subsubsection{Assessed sources of errors in GenPMAXS development.}

The work developed in this thesis yielded a deep research in reviewing and adapting the GenPMAXS methodology by means of tracking different error sources. The use of the verification against other codes was necessary to compare and identify different problems that are summarized as follows.

Issues in regard of the branch structure.

The research and development carried out in this work revealed that the default method for branching off in CASMO is done by the so called S3C case matrix option. This 
Development and validation of a multi-scale and multi-physics methodology for the safety analysis of fast transients in Light Water Reactors

branch off map is perfectly understood by the intermediate library manager between CASMO and SIMULATE, i.e. CSMLINK, but is not valid for the scheme of GenPMAXS.

The S3C branch structure defines a series of cases of combined thermal-hydraulic variables (Fuel Temperature and Coolant Density) and Control Rod compositions that are beyond the needed structure of GenPMAXS. This branch structure changes more than one variable from one state to another. This scheme generates interpolation errors when GenPMAXS changes from one state to another assuming that only one variable is changed, when in reality, the change includes another variable. The result is a wrong calculation in the addition of the corresponding partial derivatives and variable incrementrals/detrimentals.

For that purpose, the S3C case matrix was changed, defining a new case matrix that covers an adequate range of states of fuel temperature, control rod and void fraction. Table 4.1 shows an example of the branch structure that can be expected from the S3C expansion.

Table 4.1 History and branch structure in a regular distribution done by CASMO.

\begin{tabular}{|c|c|c|c|c|c|}
\hline Index & Descriptions & CR (-) & DC (kg/m3) & TF (K) & $\begin{array}{c}\text { TC } \\
(\mathbf{K})\end{array}$ \\
\hline 1 & H1 & 0 & 0.748 & 750 & 560 \\
\hline 2 & DC & 0 & 0.468 & 750 & 560 \\
\hline 3 & DC & 0 & 0.179 & 750 & 560 \\
\hline 4 & CR & 1 & 0.748 & 750 & 560 \\
\hline 5 & TF & 0 & 0.748 & 551 & 560 \\
\hline 6 & DC/TF/TC & 0 & 0.997 & 293 & 293 \\
\hline 7 & CR/DC/TF & 1 & 0.997 & 293 & 293 \\
\hline 1 & H2 & 0 & 0.468 & 750 & 560 \\
\hline 2 & DC & 0 & 0.748 & 750 & 560 \\
\hline 3 & DC & 0 & 0.179 & 750 & 560 \\
\hline 4 & CR & 1 & 0.468 & 750 & 560 \\
\hline 5 & TF & 0 & 0.468 & 551 & 560 \\
\hline 6 & DC/TF/TC & 0 & 0.997 & 293 & 293 \\
\hline 7 & CR/DC/TF/TC & 1 & 0.973 & 353 & 353 \\
\hline
\end{tabular}


Chapter 4. Validation and Verification of Codes and Methodologies for two Application cases

According to the structure of Table 4.1, branches 6 and 7 define a case where all the variables of the Description column have been changed simultaneously. The branch structure must be defined in a range that covers the maximum and minimum values of the state variables expected during the simulated transient, but as commented, the structure has to be done meeting the requirements of GenPMAXS. This means that histories must be defined, and then every history must have the same branch structure varying one state variable at once from branch to branch. Table 4.2 and Table 4.3 show a proper history and branch structure, respectively.

Table 4.2 History structure in an adequate distribution for GenPMAXS.

\begin{tabular}{|r|l|r|l|l|}
\hline PMAXS History Index & Type & CR & DC & TF \\
\hline 1 & Reference & 0 & 0.458 & 813 \\
\hline 2 & CR & 1 & 0.458 & 813 \\
\hline
\end{tabular}

Table 4.3 Branch structure in an adequate distribution for GenPMAXS.

\begin{tabular}{|r|l|r|l|l|}
\hline PMAXS Branch Index & Type & CR & DC & TF \\
\hline 1 & Reference & 0 & 0.468 & 750 \\
\hline 2 & CR & 1 & 0.468 & 750 \\
\hline 3 & DC & 0 & 0.748 & 750 \\
\hline
\end{tabular}

Assessment of the historical effects in PMAXS libraries.

The branch structure is defined by the user in GenPMAXS input file. In addition, this input file defines the base case and how is to be read the data from the Transport Lattice code that will be used to generate the PMAXS library. GenPMAXS can adapt its scheme for reading several Transport Lattice codes. Due to the framework with KKL, different calculations were done with CASMO in their facility. KKL uses the suit CASMO/SIMULATE for core follow calculations. Their source inputs for such calculations define a high-detailed core with all the different advanced fuel lattices and the different control rod compositions. Therefore, the cross-section data was generated for a wide span of different fuel lattices plus several control rod compositions. This fact yields the option of accounting different historical values for control rods.

The work in regards of the Cross-Section Libraries for the proposed methodology drove to different test cases and source debugging that revealed a limitation in PARCS code. PARCS (due to the implemented PMAXS scheme) can interpolate the historical values of the cross-section for different branches of fuel temperature and coolant density. Conversely, it cannot interpolate within different historic values of control rod compositions.

The result is that there are nodes in the 3D mesh of PARCS model that are affected by the historic effect of different control rod compositions. Since PARCS can only take the branches of the first control rod composition, such mentioned nodes will have a deviation 
Development and validation of a multi-scale and multi-physics methodology for the safety analysis of fast transients in Light Water Reactors

from the reference, since the absorption cross-section will not be properly calculated. The comparisons that section 3.2.4 shows give credit to this situation.

For further applications, it is necessary to approach this issue from the safety analysis point of view. The engineering decision can be though conservative. A sensitivity analysis can clarify the effect of control rod worth in the simulated transient cases. A considered low correlation can be accepted for not accounting the discrepancies generated by this issue. On the other hand, if the sensitivity to this issue is significant, the control rod composition with least worth can be assumed as the single rod composition accepted by PARCS.

Limitation of PARCS for weighting multiple Cross-Section Libraries in one single mesh node.

It is common to define the calculation space of a reactor core as a 3D mesh of regular nodes. The regular size of a node, and the one used for the different calculations in this thesis work, is a cube of $15.24 \mathrm{~cm}$ long. When dividing a fuel assembly in different regions where the homogenization of the cross-section is done (see Figure 4.3), it is not always possible to match the limits of a fuel region and the nodal distribution of the core simulator mesh. Figure 4.3 depicts the commented situation.

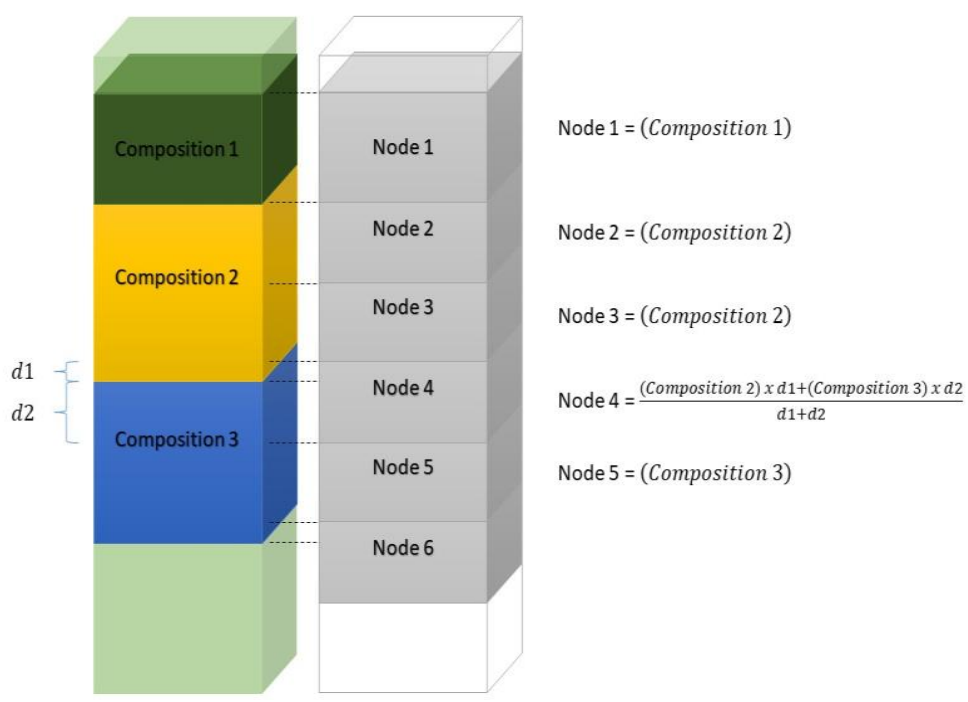

Figure 4.3 Mismatch in the nodal distribution and the regions of the different Cross-Section Libraries.

This issue can be solved by weighting the cross-section data sets with the volume of the node. The SIMTAB methodology has approached this issue by editing the Cross-Section Libraries with a pre-defined input locating which cross-section data sets must be 
Chapter 4. Validation and Verification of Codes and Methodologies for two Application cases

weighted. Regarding GenPMAXS, it is not possible to undertake this weighting externally from both codes sources of GenPMAXS and PARCS.

Again, prior to the corresponding code modification by the code developers, a valid approach for this issue is to adapt the $3 \mathrm{D}$ mesh of the core simulator to the geometrical requirements of the fuel assembly regions. This can be a cumbersome task, and may not be allowed if the core simulator does not consider irregular axial node distributions for the radially distributed nodes. In this case, it is necessary to adapt the Transport Lattice Code to homogenize the core regions coherently to a regular nodal distribution in the core simulator.

Issues in the specific use of CASMO feeding GenPMAXS with cross-section data.

GenPMAXS uses the output generated by a Transport Lattice code to re-arrange and undertake the corresponding derivatives for the collapsed and homogenized cross-section data. The complexity and size of the problem depend on the size of the branch structure, the homogenized core regions and the exposure (burnup) level of the case. These dimensions yield a number of so-called state points in CASMO. The approached transport equation is solved for each lattice in CASMO, for the defined burnup steps and the combination of state variables that conform the branch structure. Calculations for fresh fuel core, for instance, does not need further calculations in the burnup dimension. Therefore, the number of state points in CASMO is significantly reduced. Nevertheless, in order to provide complete Cross-Section Libraries, it is advisable to include the full life of fuel assemblies in the calculated regions. This fact, added to the necessary size of the branch structure yield a high number of state points to be calculated.

Lattice code as CASMO, use memory arrays to store the results of the calculations. Special attention must be given to the run-time error notifications in order to assure a complete calculation of every programmed state point. A failure during the calculation sequence cannot assure that the cross-section data is coherent up to certain burnup steps after the error notification. The following section show the results for the first fuel cycle of KKL core, where the calculation of the cross-section data with CASMO is assured to be completed.

\subsubsection{Current results with GenPMAXS methodology.}

The development of this thesis work took place in the framework of collaborating research project between ISIRYM-UPV and KKL. Due to this, different simulation tools like CASMO/SIMULATE suite HELIOS (from Studsvik Scanpower) where able to be used in the facilities of KKL as license owner. The results of the research of this part of the proposed methodology have been obtained in collaboration with KKL and the use of the codes was under their supervision in their facility.

As commented, the proposed methodology in this thesis includes a step in charge of generating the cross-section data for further calculations in other steps. This step gener- 
Development and validation of a multi-scale and multi-physics methodology for the safety analysis of fast transients in Light Water Reactors

ates the so-called Cross-Section Libraries in a specific format. In general terms, the libraries are used as an input for the corresponding Diffusion Code and therefore, the format and contents of the Cross-Section Libraries have to be adapted to the used Diffusion Code. Regarding this methodology, the Diffusion Code solving the neutron kinetics is PARCS and the Cross-Section Libraries used are the PMAXS libraries, generated by the interface GenPMAXS. In addition, as a support methodology for Cross Section Libraries management, the thesis work counts on the SIMTAB methodology for this step.

The procedure of this part of the proposed methodology takes advantage of the transport lattice codes used in KKL. The staff in charge of the Core Follow Design and Management use codes such as CASMO, SIMULATE and HELIOS. The database of these codes in KKL contains the corresponding information that GenPMAXS and SIMTAB use to retrieve the Cross Section data and provide the two-group cross section sets that PARCS needs for transient calculations. However, specific development work has been done in order to assure a correct information flow from the aforementioned lattice codes. A significant percentage of the total effort invested in this project has been headed to the adaptation of the GenPMAXS methodology to the database of the core follow. This issue has been found due to the lack of scientific work and literature regarding the development of the PMAXS files. Figure 4.4 shows the flow of information and the related codes of this part of the methodology. Notice that the development of this step works in parallel with the corresponding code-to-code verification in order to assure the quality of the process. This code-to-code verification is realized using the equivalent codes that solve the diffusion equation using as input the results predicted by CASMO or HELIOS. For instance, the results predicted by the suite CASMO/GenPMAXS/PARCS are equivalent to the predicted by CASMO/SIMULATE. Consequently, for the code-to-code verification, a steady-state case of the core of KKL is modelled, and the results are compared, using as figure of merit the k-effective parameter, and the axial and radial power distributions. 


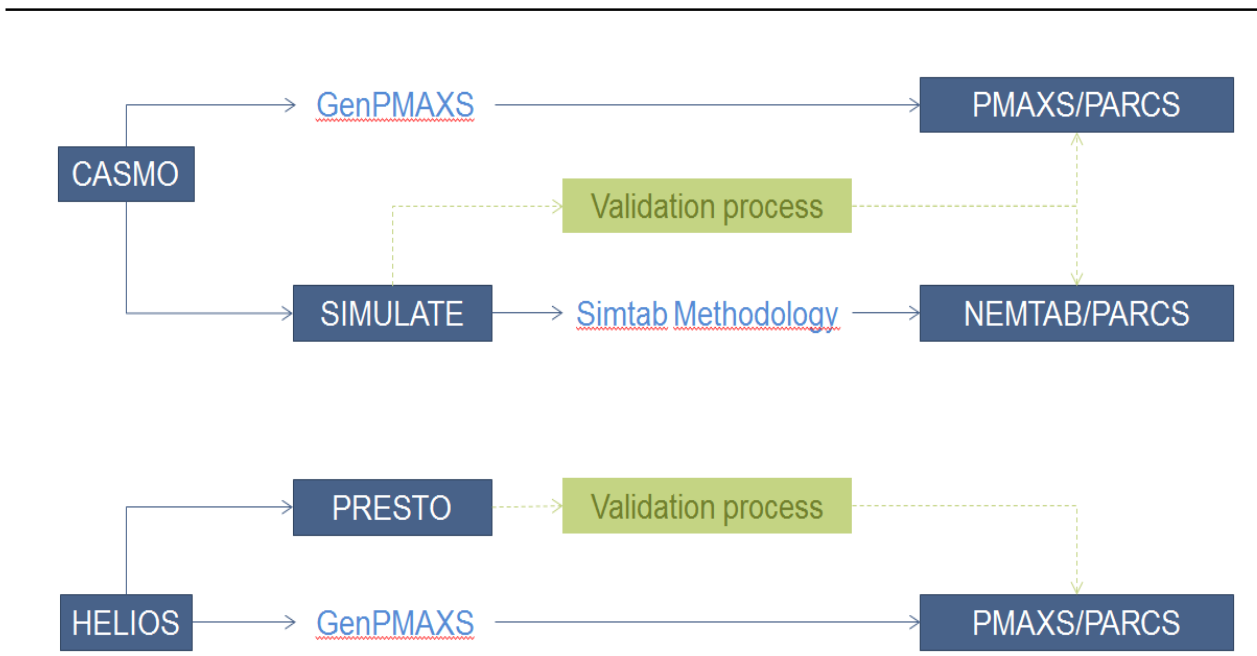

Figure 4.4 Data flow for the generation and verification of Cross Section Libraries.

The following subsections report the status related to the Cross Section Libraries generation using both methodologies, GenPMAXS and SIMTAB.

The proposed methodology uses the neutron kinetics code PARCS to solve the diffusion equation and hence introduce the feedback in/from the thermal-hydraulic calculations of the core behavior. For this purpose, the corresponding cross-section data have to be provided, so PARCS can solve the diffusion equation at each core node. This Cross Section data is arranged in core segments. Each segment works as a library containing neutronic data such as fission cross section, scattering cross section and on. These data vary depending on different variables. The variables determining the cross section information used in this methodology are the fuel temperature, the coolant density, the control rod insertion and the burnup. These segments are obtained from the homogenization of the cross sections in two energy groups collapsed for core regions considered uniform. For example, a very uniform fuel assembly can be defined as a core segment, but if a fuel assembly has two partial rod length types, it can be defined in three different segments. In addition, the evolution of the cross section data along the burnup process may differ if the fuel assembly has been operating near a control rod, or near the outer ring of the core. This means that the same fuel assembly can be defined with different core segments, not in geometry, but in operation history.

Consequently, there are two ways to define the spatial distribution of the core. One way is based on the geometry, where the core defines the nodes where the calculations are going to take place. The other is the segment distribution. Figure 4.5 shows the different distributions in the core (left). Further subsections will explain the issues found due to the different ways of distributing the core regions. As Figure 4.5 (right) shows, each node is marked in the cell grid. On the other hand, it can be appreciated different color set for 
Development and validation of a multi-scale and multi-physics methodology for the safety analysis of fast transients in Light Water Reactors

certain groups of nodes. The nodes sharing color set mean that are sharing the same segment, i.e. the same neutronic composition, although two segments could superpose one node.
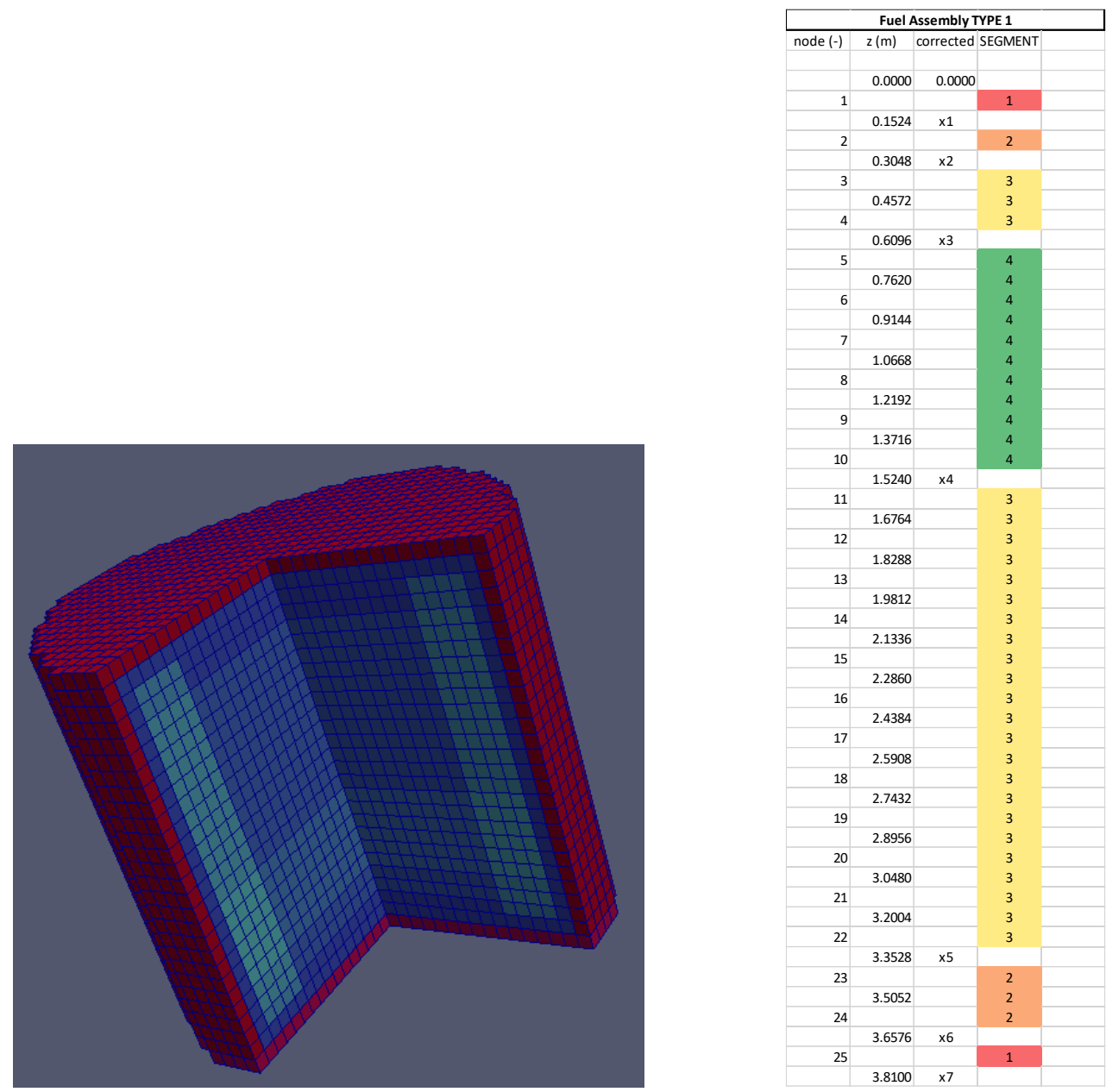

Figure 4.5 Core distribution in geometrical nodes (left) and in segments (right).

Figure 4.5 (right) shows the nodal distribution and how the different fuel segments can be fit in the distribution. The example of Figure 4.5 (right) corresponds to the fuel assembly TYPE 1 of fuel cycle 01 . For this case, the length of the segments matches with the nodal distribution. For instance, nodes 3 and 4 have the assignment of segment 3. However, it can occur that the length of a segment does not fit with the nodal distribution, and one node has to share the axial length with two segments. This fact will require a 
weighting of the neutronic data of the segment according to the axial length of the node, in order to correct this effect.

The GenPMAXS interface uses data from the output results of different lattice transport codes. For the purpose of the proposed methodology, CASMO and HELIOS are considered for providing the corresponding data to GenPMAXS. Figure 4.6 defines the flow of information of this step of the methodology.

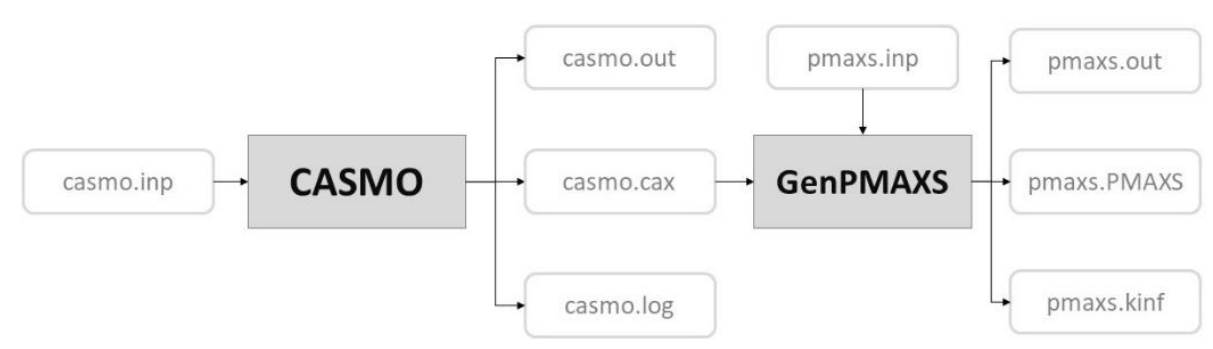

Figure 4.6 File flow between CASMO and GenPMAXS.

At the first moment, both data sources where being developed, however the flexibility of HELIOS features adds a significant complexity to the problem and hence, the work force was headed to the suite CASMO/PMAXS.

The definitive results of the GenPMAXS methodology have achieved an acceptable error rate in the code-to-code verification. The following figures and tables displayed in this subsection will show the comparison of the figures of merit used to the verification process, namely k-effective comparison, radial and axial distribution. The results of PMAXS/PARCS are compared to the SIMULATE3 equivalent simulation.

The previous work to track the error sources in the PMAXS/PARCS results headed the simulation exercise to simpler cases. As a result, the work is made on fuel cycle 01 since is the less complex real case available. Notice that fuel cycle 01 uses only 7 core segments with one control rod type. After solving the issues commented previously (see Section 3.2.3) the comparison at the End Of Cycle 01 core composition was realized. The measures and solutions adopted are summarized as follows:

- Use of control rod composition COMPOSITION1 as CRD and HNDL as CR1 in CASMO4 input files. This allows a correct HCR 3D MAP values in SIMULATE3.

- Definition of branches and histories as in tables 1 and 2. Different reduction of case matrix branches was attempted in order to reduce the computational cost and the file size, the results showed significant error. 
Development and validation of a multi-scale and multi-physics methodology for the safety analysis of fast transients in Light Water Reactors

- Reduction of burnup steps until $20 \mathrm{GWd} / \mathrm{MTU}$. This is made because no higher burnup is detected in the End Of Fuel Cycle 01, so it was not necessary enlarge the size of the resulting .cax files.

- The rotational ADF matrix has been redefined and the reflector PMAXS use the corrected assembly discontinuity factors.

Figure 4.7 shows the control rod bank configuration at this burnup step of the fuel cycle.

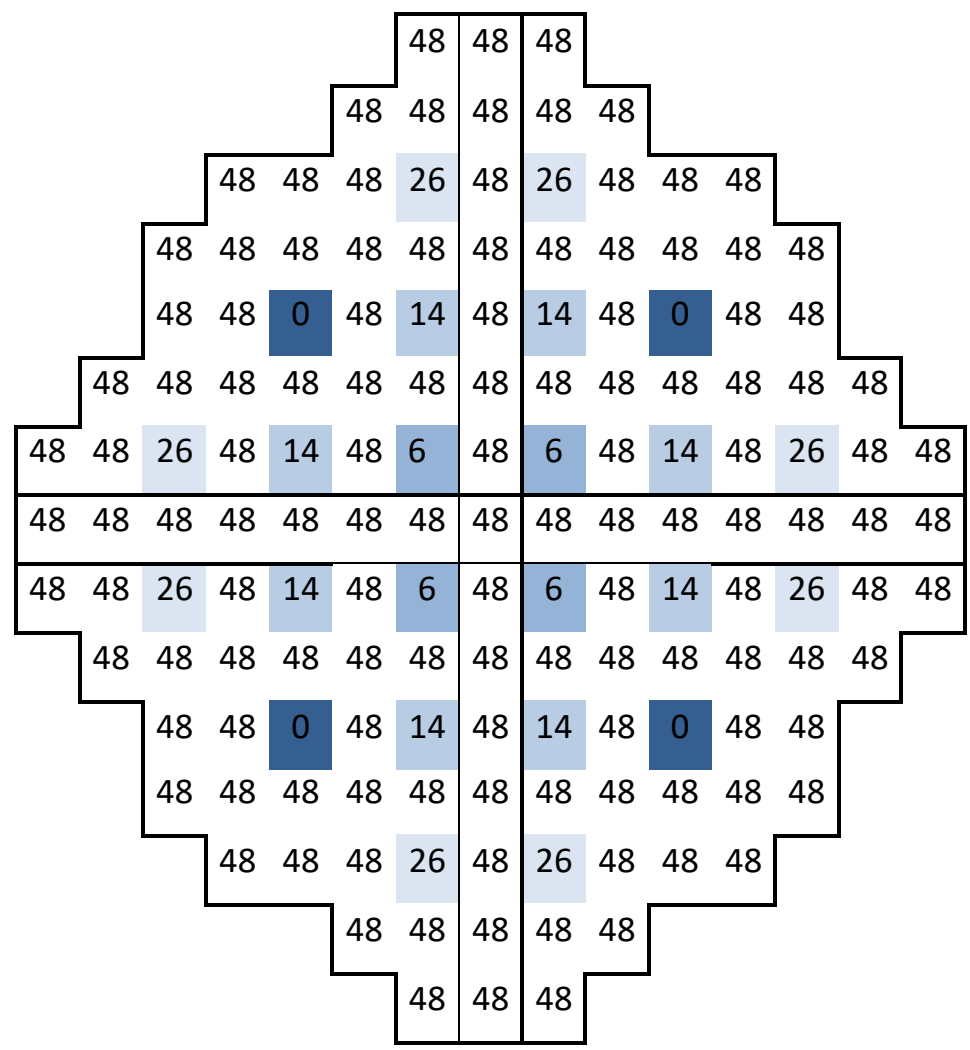

Figure 4.7 Control rod configuration in fuel cycle 01 .

Figure 4.8 shows the axial power profile comparison and Figure 4.9 shows the radial relative error of the power comparison between CASMO4/SIMULATE3 and PMAXS/PARCS results. The PMAXS/PARCS graphic in Figure 4.8 is labeled as PARCS indicating the code version, adding the label PMAXS indicating that the PMAXS libraries have been generated using the SIMULATE files that assign the CRD 
Chapter 4. Validation and Verification of Codes and Methodologies for two Application cases

label to the corresponding active composition of the control rod, instead of the handle composition.

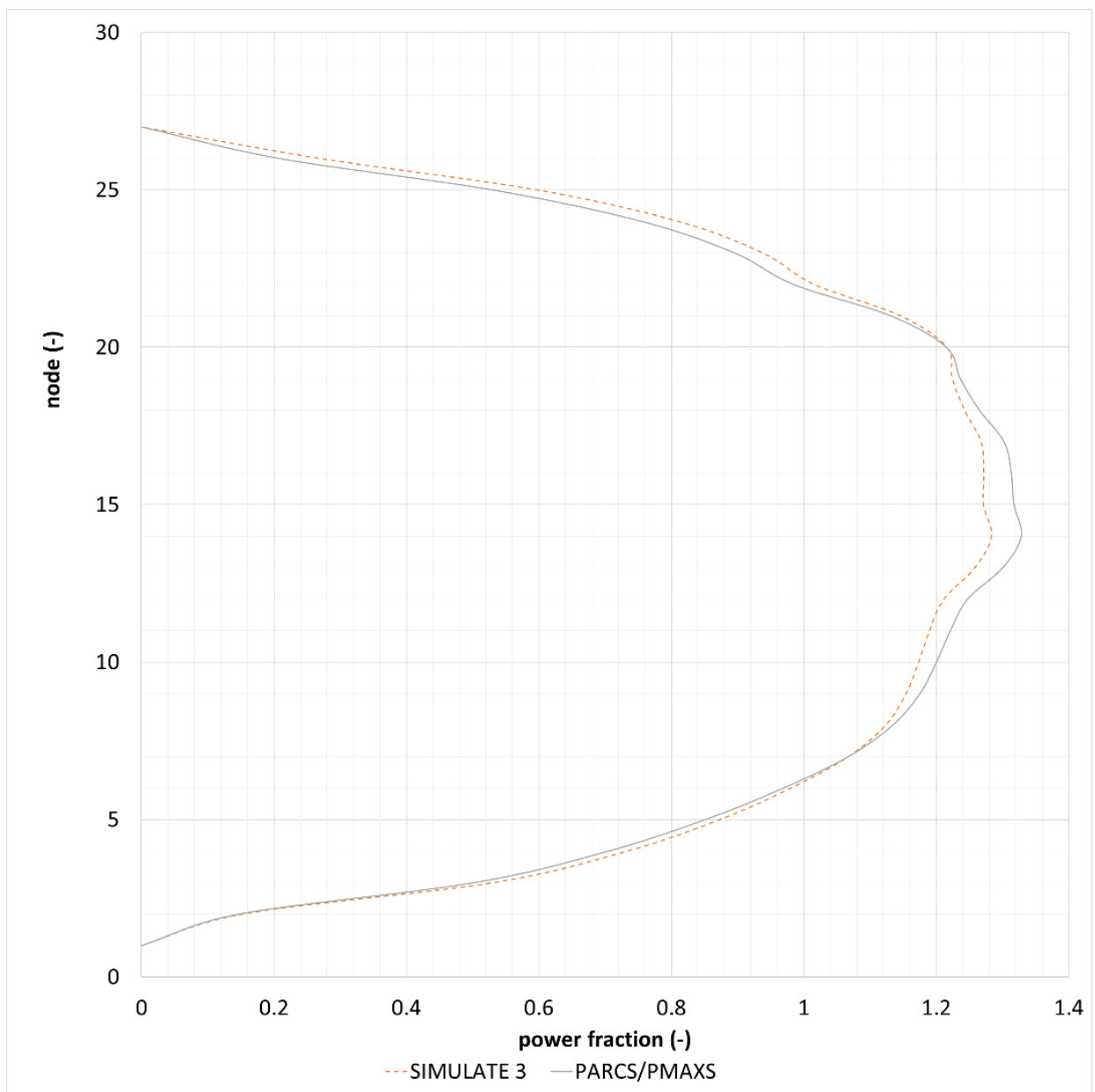

Figure 4.8 Axial power profile comparison: PMAXS/PARCS vs CASMO4/SIMULATE3 (C01EOC). 
Development and validation of a multi-scale and multi-physics methodology for the safety analysis of fast transients in Light Water Reactors

$\begin{array}{llllll}1.73 & 0.75 & 0.67 & 0.72 & 0.81 & 1.69\end{array}$

$\begin{array}{lllllllllll}2.31 & 1.41 & 1.24 & 1.10 & 1.14 & 1.07 & 1.12 & 1.33 & 1.51 & 2.31\end{array}$

$\begin{array}{lllllllllllll}3.01 & 1.35 & 1.21 & 0.77 & 0.68 & 0.60 & 0.70 & 0.75 & 0.76 & 1.22 & 1.36 & 3.04\end{array}$

$\begin{array}{lllllllllllllllll}2.61 & 2.10 & 0.41 & 1.05 & 0.71 & 0.56 & 0.42 & 0.38 & 0.40 & 0.40 & 0.54 & 0.73 & 1.13 & 0.35 & 1.98 & 2.48\end{array}$

$\begin{array}{lllllllllllllllllllll}2.67 & 1.59 & 1.35 & 1.09 & 0.68 & 0.49 & 0.10 & 0.10 & 0.25 & 0.19 & 0.10 & 0.12 & 0.48 & 0.68 & 1.07 & 1.36 & 1.74 & 2.91\end{array}$

$\begin{array}{lllllllllllllllllllll}2.93 & 1.71 & 1.08 & 0.88 & 0.71 & 0.51 & 0.26 & 0.04 & -0.47 & -0.11 & -0.15 & -0.51 & 0.00 & 0.25 & 0.54 & 0.71 & 0.86 & 1.11 & 1.65 & 3.04\end{array}$

$\begin{array}{llllllllllllllllllllllllll}2.71 & 1.62 & 1.03 & 0.86 & 0.64 & 0.58 & 0.05 & 0.19 & -0.33 & 0.00 & -0.35 & -0.38 & 0.02 & -0.29 & 0.20 & 0.05 & 0.56 & 0.65 & 0.82 & 1.11 & 1.71 & 2.74\end{array}$

$\begin{array}{llllllllllllllllllllllll}2.28 & 1.49 & 1.02 & 0.82 & 0.63 & 0.46 & -0.20 & 0.22 & -0.38 & -0.23 & -0.63 & -0.54 & -0.50 & -0.64 & -0.18 & -0.41 & 0.25 & -0.20 & 0.51 & 0.62 & 0.82 & 1.07 & 1.58 & 2.55\end{array}$

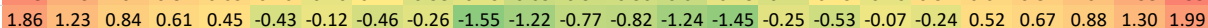

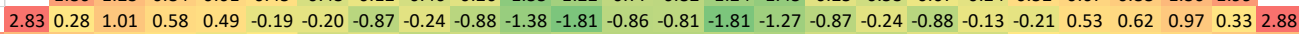

$\begin{array}{llllllllllllllllllllllllllll}2.12 & 1.23 & 0.94 & 0.52 & 0.35 & -0.08 & 0.20 & -0.51 & -0.26 & -0.75 & -0.56 & -1.06 & -0.79 & -0.88 & -0.90 & -0.82 & -1.02 & -0.50 & -0.70 & -0.24 & -0.47 & 0.25 & -0.01 & 0.47 & 0.57 & 0.95 & 1.19 & 1.95\end{array}$

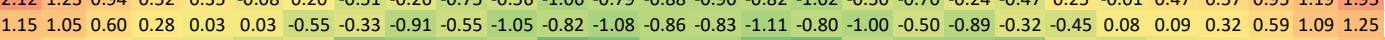

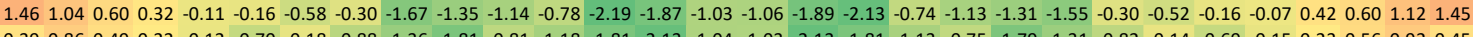
$\begin{array}{lllllllllllllllllllllllllllllll}0.39 & 0.86 & 0.49 & 0.22 & -0.12 & -0.70 & -0.18 & -0.88 & -1.36 & -1.81 & -0.81 & -1.18 & -1.81 & -2.13 & -1.04 & -1.03 & -2.13 & -1.81 & -1.13 & -0.75 & -1.79 & -1.31 & -0.82 & -0.14 & -0.69 & -0.15 & 0.23 & 0.56 & 0.92 & 0.45\end{array}$

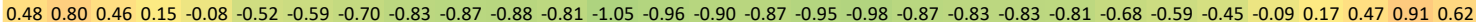

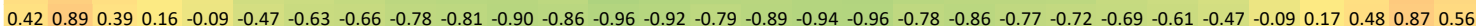

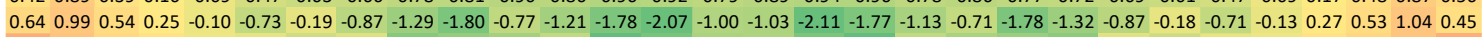

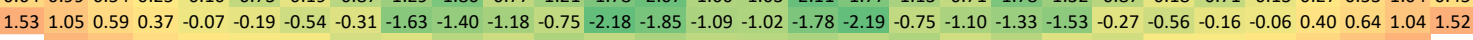

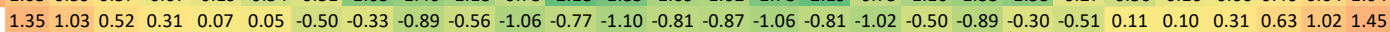

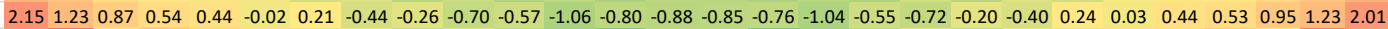

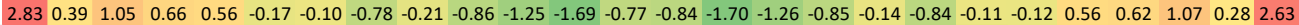

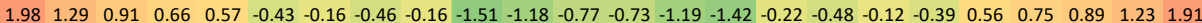

$\begin{array}{lllllllllllllllllllllllll}1.98 & 1.29 & 0.91 & 0.66 & 0.57 & -0.43 & -0.16 & -0.46 & -0.16 & -1.51 & -1.18 & -0.77 & -0.73 & -1.19 & -1.42 & -0.22 & -0.48 & -0.12 & -0.39 & 0.56 & 0.75 & 0.89 & 1.23 & 1.91 \\ 2.41 & 1.59 & 1.11 & 0.80 & 0.64 & 0.46 & -0.14 & 0.24 & -0.39 & -0.17 & -0.57 & -0.45 & -0.50 & -0.57 & -0.19 & -0.36 & 0.28 & -0.18 & 0.55 & 0.68 & 0.83 & 1.11 & 1.60 & 2.41\end{array}$

$\begin{array}{llllllllllllllllllllllllll}2.79 & 1.66 & 1.02 & 0.81 & 0.62 & 0.60 & 0.07 & 0.22 & -0.26 & 0.01 & -0.37 & -0.35 & 0.07 & -0.20 & 0.28 & 0.11 & 0.59 & 0.67 & 0.87 & 1.09 & 1.71 & 2.88\end{array}$

$\begin{array}{llllllllllllllllllllllll}2.80 & 1.74 & 1.05 & 0.90 & 0.68 & 0.58 & 0.28 & 0.11 & -0.40 & -0.02 & -0.10 & -0.41 & 0.12 & 0.29 & 0.60 & 0.69 & 0.94 & 1.08 & 1.73 & 2.96\end{array}$ $\begin{array}{llllllllllllllllllll}2.93 & 1.65 & 1.37 & 1.09 & 0.70 & 0.52 & 0.19 & 0.10 & 0.30 & 0.30 & 0.16 & 0.21 & 0.55 & 0.72 & 1.13 & 1.31 & 1.70 & 2.91\end{array}$ $\begin{array}{llllllllllllllll}2.71 & 2.02 & 0.45 & 1.07 & 0.81 & 0.55 & 0.49 & 0.43 & 0.43 & 0.48 & 0.59 & 0.77 & 1.14 & 0.50 & 2.12 & 2.54\end{array}$

$\begin{array}{llllllllllllll}3.01 & 1.42 & 1.18 & 0.80 & 0.69 & 0.63 & 0.63 & 0.74 & 0.80 & 1.20 & 1.44 & 3.01\end{array}$

$\begin{array}{lllllllllll}2.21 & 1.39 & 1.30 & 1.12 & 1.09 & 1.14 & 1.10 & 1.30 & 1.44 & 2.17\end{array}$

$\begin{array}{llllll}1.80 & 0.72 & 0.87 & 0.64 & 0.75 & 1.73\end{array}$

Figure 4.9 Radial error distribution (\%) of the power comparison: PMAXS/PARCS vs CASMO4/SIMULATE3 (C01EOC).

Figure 4.9 shows a symmetric distribution of the error and no error introduced by the nodes with inserted control rods. This is verified as follows with the results of the ARO (All Rods Out) case. Figure 4.10 and Figure 4.11 show the axial and radial comparison, and the results are summarized in Table 4.4. 
Chapter 4. Validation and Verification of Codes and Methodologies for two Application cases

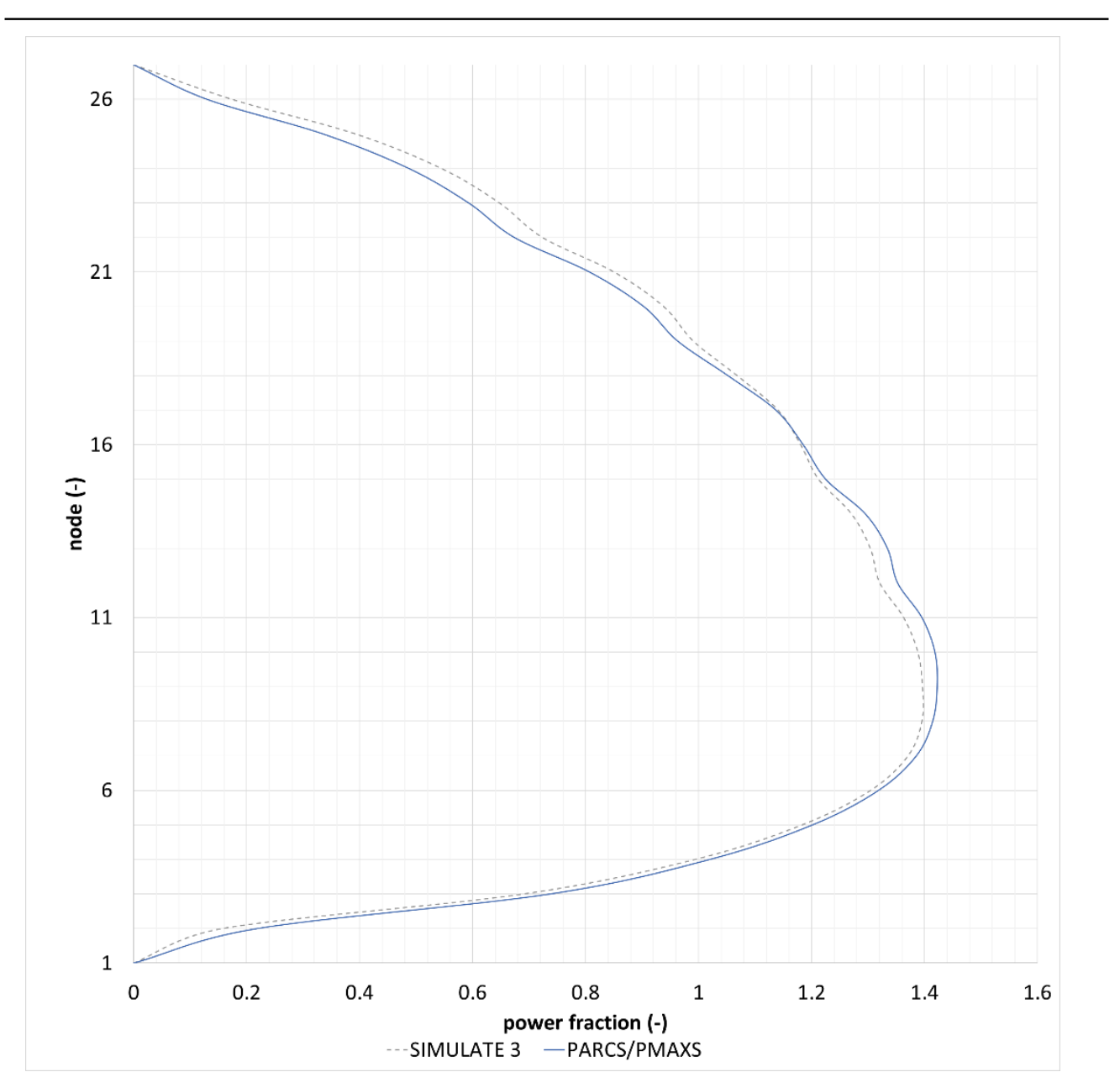

Figure 4.10 Axial power profile comparison: PMAXS/PARCS vs CASMO4/SIMULATE3 (CO1EOC-ARO). 
Development and validation of a multi-scale and multi-physics methodology for the safety analysis of fast transients in Light Water Reactors

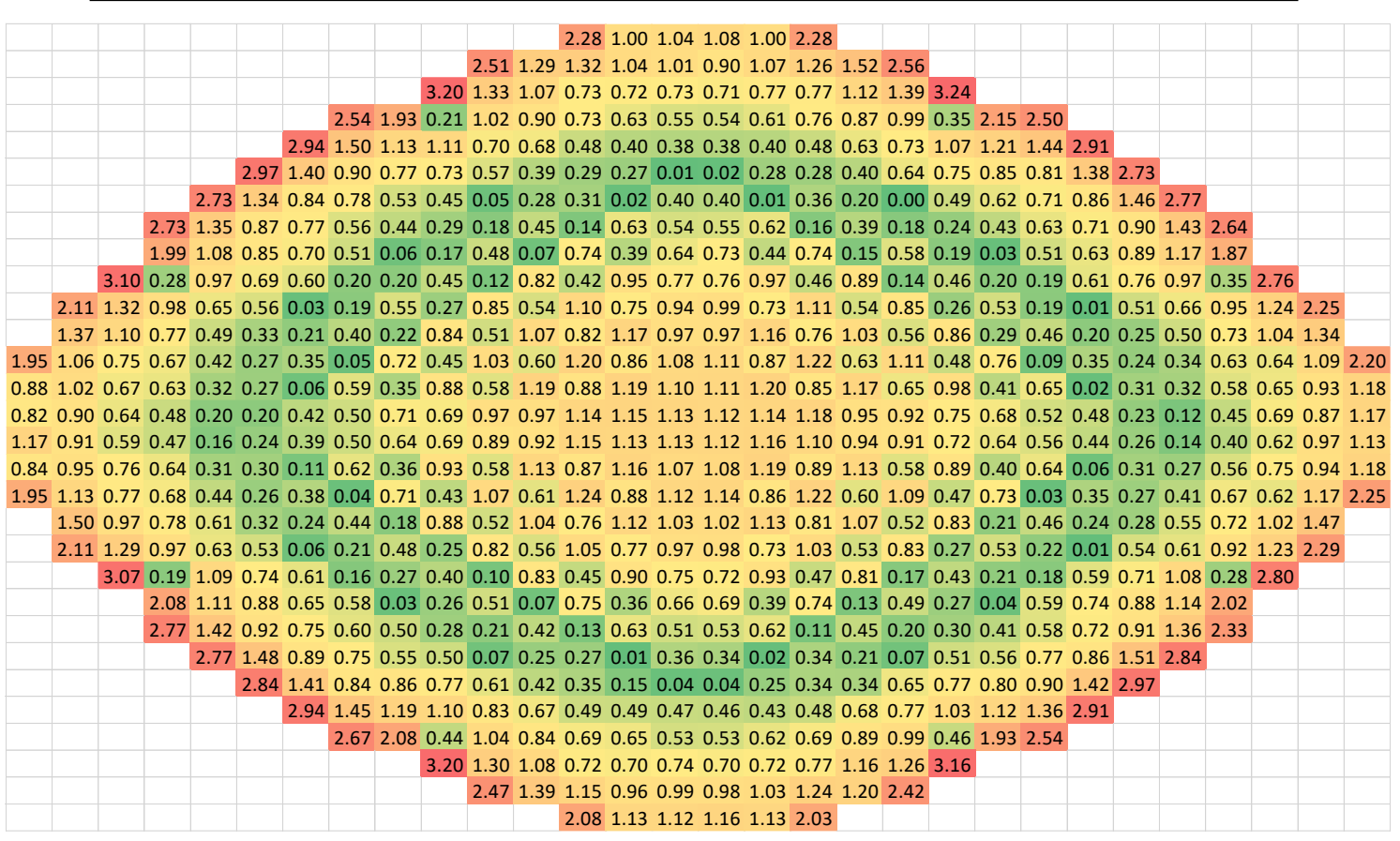

Figure 4.11 Radial error distribution (\%) of the power comparison: PMAXS/PARCS vs CASMO4/SIMULATE3 (CO1EOC-ARO).

Table 4.4 Summary of comparison results for case EOC01: PMAXS/PARCS vs CASMO4/SIMULATE3.

\begin{tabular}{|l|l|l|l|}
\hline CASE & $\begin{array}{l}\text { Axial power pro- } \\
\text { file error }- \text { RMS } \\
(\%)\end{array}$ & $\begin{array}{l}\text { Radial power profile } \\
\text { error - RMS }(\%)\end{array}$ & $\begin{array}{l}k \text {-effective } \\
(\mathrm{pcm})\end{array}$ \\
\hline EOC01 & 3.45 & 0.84 & 23.0 \\
\hline ARO & 3.54 & 0.77 & 26.5 \\
\hline
\end{tabular}

Notice that the errors observed in Table 4.4 are slightly smaller. However, it can be concluded that the error rate introduced by the control rods has been reduced a lot.

\subsubsection{Conclusions and future work for GenPMAXS methodology.}

The efforts invested in the development of the GenPMAXS methodology have been enough to reduce the errors to acceptable limits. The solutions implemented include the control rod definition in CASMO4 and the adequate case matrix definition for CASMO4 
input files. Nonetheless, it is important to account the following aspect. The results are showed for the first cycle, where the less heterogeneous compositions are expected. Further fuel cycle simulations will be more complex, and hence an error rate introduction is expected.

Moreover, it has to be noticed that the code-to-code verification is made assuming SIMULATE3 methods as a reference. Some of the differences in PARCS are justified and can be assumed as correct and therefore, it is necessary to consider that the correct results are not directly related to reduce the error rate of the comparison with SIMULATE3.

Regarding ongoing work, further case matrix definitions are headed to reduce the number of branches so the .cax files and hence the PMAXS files have smaller size without increasing the consequent interpolation errors. In addition, it has to be accounted the limitation of the Coolant density for moderator temperatures below the saturation temperature. The simulation can include the existence of subcooled water entering the reactor core. In this case, the cross-section library will account branches at void 0 but with different density values. This fact increases the number of branches, and hence it adds a computational cost that can be hardly avoided.

The aim of this part of the methodology is to develop a full PMAXS library containing all the segments that made the core of KKL along the NPP life. This will make possible to define the neutronic part of the core for any transient case that is wanted to be simulated. For this purpose, further code-to-code verifications are in progress: one for the Turbine Trip core configuration of Fuel Cycle 18, and two more for the instability tests of fuel cycles 07 and 10. However, as it has been commented, more recent fuel cycles increase the complexity of the data stored in the PMAXS libraries, increasing the computational cost and adding difficult to the handling of the PMAXS files, as Table 4.5 shows.

Table 4.5 File size and computational time needed for fuel cycle calculations with CASMO4.

\begin{tabular}{|l|l|l|l|}
\hline Fuel Cycle & $\begin{array}{l}\text { Number of seg- } \\
\text { ments (-) }\end{array}$ & $\begin{array}{l}\text { Maximum segment } \\
\text { size (cax file) }\end{array}$ & $\begin{array}{l}\text { Maximum compu- } \\
\text { tational time } \\
\text { needed (hours) }\end{array}$ \\
\hline 01 & 7 & $785 \mathrm{MB}$ & 48 \\
\hline 07 & 24 & $1.50 \mathrm{~GB}$ & 164 \\
\hline 18 & 47 & $2.60 \mathrm{~GB}$ & 543 \\
\hline
\end{tabular}


Development and validation of a multi-scale and multi-physics methodology for the safety analysis of fast transients in Light Water Reactors

\subsection{Validation of void fraction correlation in CTF-UPVIS against the PSBT Benchmark of the OECD/NEA.}

The introduction of this chapter summarizes the importance of code validation. In the quality assessment of a simulation tool for its further qualification is necessary to assess the implemented physics against real data. This data comes usually from experimental facilities that either model specific phenomena or the integral behavior of a NPP part or reactor type. This section intent to contribute to the thesis work in one of the validation processes that have been undertaken with the objective of complete the BE feature of the proposed methodology for safety analysis.

The re-evaluation of the power capabilities and lifespan of the Nuclear Power Plants (NPP) required a more accurate prediction of the safety variables in order to not compromising the operation safety and enhance the cost-effectivity of such operation. Moreover, the advances in computer technology helped to solve more complex and large numerical problems. The result is an evolution of the simulation of transient and steady state scenarios focused in more realistic results, by means of finer scales, detailed physical correlations and accounting the interaction between the different physics playing a relevant role in the nuclear core behavior. This is the so called Best Estimate (BE) approach. Nevertheless, a significant level of accuracy needs to account the propagation of the uncertainties along the different calculation processes inherent to the programmed models and to the measured parameters used as input. Considering the uncertainty of the problem defines the boundaries of the predicted values and hence, can be used for evaluating the safety margins.

This section presents the validation of a BEPU (Best Estimate Plus Uncertainty) methodology that combines the use of a BE code and the use of a toolkit for the Uncertainty Quantification (UQ) and the Sensitivity Analysis (SA). The code that will be used is CTF-UPVIS, a thermal-hydraulic sub-channel code developed at ISIRYM/UPV based on COBRA-TF. CTF-UPVIS analyses the behavior of the coolant in rod arrays by solving the conservation equations for mass, energy and momentum in bi-phasic flow for two fluids.

The performance of CTF-UPVIS is complemented using the DAKOTA toolkit. DAKOTA is a code developed at Sandia National Laboratories for the uncertainty quantification and the sensitivity analysis. The aim of this code is to define the sample size to undertake a statistical evaluation according the target probability of the results and the interval of confidence. The optimization of this problem is not trivial since several simulations have to be done looking forward the most efficient use of computational resources.

Different safety and operation variables can be predicted for an optimal evaluation of the core behavior. In this section, the author focus in the prediction of the void fraction in order to evaluate the implemented models of CTF-UPVIS and to validate the BEPU methodology. For these purposes, the BEPU methodology is applied to the experimental 
Chapter 4. Validation and Verification of Codes and Methodologies for two Application cases

results of the NEA/OECD PSBT tests (Rubin, 2010).Description of the validated methodology.

The resulting work is based on previous exercises at ISIRYM for the evaluation of CTFUPVIS and the BEPU methodology (P. Hidalga, 2018). The presented results will show, on the one hand, the analyses of the propagation of the uncertainty existing in different input variables and the effect on the uncertainty of a target output variable, evaluated by the BEPU methodology. On the other hand, this section reports the application of the conclusions of previous work such as neglecting the uncertainty of the power distribution. In addition, new models in the prediction of the void fraction are validated for this exercise. The predicted void fraction at certain bundle levels is selected as target output variable due to its relevance regarding safety analysis in LWRs.

The $2 \sigma$ criterion is used to evaluate the uncertainty of the predicted void fraction. For this purpose, the authors have based the analysis in the Wilks' formula, in order to estimate the sample size that allows meeting the $95 / 95$ criterion. This means that with a sample of 146 cases, it is possible to assure with a $95 \%$ of probability that the real value of the predicted variable would be inside the Confidence Interval, defined within the $95 \%$ of the sample distribution. In case of having a normal distribution for the output variable, this $95 \%$ CI could be calculated as Equation 4.3, being both $\sigma_{s}$ and $\mu_{s}$ the sample's standard deviation and mean value, respectively.

$$
C I=\mu_{s} \pm 1.96 \cdot \sigma_{s}
$$

Equation 4.3

Nevertheless, the PDF of the predicted output value may not be normal and therefore, it would not be possible to define the $\mathrm{CI}$ as in Equation 4.3. For other cases, it is necessary to define the upper and lower boundaries with the maximum and minimum values of the sample. In this case, according to Wilks theory, it is possible to assure with a $95 \%$ of probability that the real value of the predicted variable falls in the region defined by the sample boundaries. The Wilks' formula is showed in Equation 4.4, where $\alpha$ is the uncertainty, $\beta$ the statistical confidence and $\mathrm{n}$ the sample size. It has to be notice that this formula is defined for analysis where 3 different output variables and double tolerance is accounted.

$$
1-\alpha^{n}-n(1-\alpha) \alpha^{(n-1)} \geq \beta
$$

Equation 4.4

According to Equation 4.4, the resulting sample size is of 146. The selected Test Runs of the benchmark have been sampled by means of varying the selected input variables according to their Probability Density Functions (PDF). The 146 samples for each of the 
Development and validation of a multi-scale and multi-physics methodology for the safety analysis of fast transients in Light Water Reactors

11 selected tests where run in the cluster of the ISIRYM and the results where postprocessed with DAKOTA toolkit to provided further UQ and SA. Notice that the sample size of 146 cases applies for one single output variable. In the presented application case, 3 dependent variables are analyzed, and therefore the 146 cases are sampled for one of each.

The DAKOTA pre-processor tool generates a perturbation matrix of the target input variables regarding the sample size and their PDF. The selection of the input variables, as well as their PDFs, have been chosen according to the available scientific data. The information about the input parameters is showed in Table 4.6 and has been retrieved from the Benchmark for Uncertainty Analysis in Modelling (UAM) documentation (Blyth et al., 2013). According to UAM report, the mean value has been normalized to 1.0, and the accuracy is provided in terms of $\pm 2 \sigma$, except for the rod diameter having $\pm 3 \sigma$, and the Inlet Coolant Temperature which is defined in terms of a uniform distribution. Regarding this last input parameter, CTF-UPVIS uses as input the coolant enthalpy and the uncertainty information given in the specifications of the benchmark have been adapted to provide CTF-UPVIS with the equivalent enthalpy values.

According to the input variables, each of the samples will have a perturbed set of the input variables that will be introduced automatically in each input deck of CTF-UPVIS. A Linux based script was used to automatize the simulation of the cases, and afterwards the results were evaluated with the DAKOTA post-processing tool. Moreover, DAKOTA allows quantifying the SA by means of providing correlation coefficients. The SA is realized by calculating the PRCC (see section 3.3.7). These correlations are used to identified the separate effect of each variable, accounting the non-linearity of the inputs and the output. 
Chapter 4. Validation and Verification of Codes and Methodologies for two Application cases

Table 4.6 Uncertainty definition of the input variables for the PSBT simulation.

\begin{tabular}{|l|l|l|l|l|l|}
\hline Definition & Variable & PDF & $\begin{array}{l}\text { Accu- } \\
\text { racy } \\
\mathbf{( \% )}\end{array}$ & Mean & $\begin{array}{l}\text { Standard } \\
\text { Deviation }\end{array}$ \\
\hline Outlet core pressure & press & Normal & 1.0 & 1.0 & 0.0033 \\
\hline Inlet mass flow & mflow & Normal & 1.5 & 1.0 & 0.0050 \\
\hline $\begin{array}{l}\text { Bundle power } \\
\text { Equilibrium distribu- } \\
\text { tion weighting factor in } \\
\text { the void drift }\end{array}$ & aaak & Normal & 14.0 & 1.0 & 0.0700 \\
\hline $\begin{array}{l}\text { Two-phase multiplier } \\
\text { of the mixing coeffi- } \\
\text { cient }\end{array}$ & thetm & Normal & 24.0 & 1.0 & 0.1200 \\
\hline $\begin{array}{l}\text { Single-phase mixing } \\
\text { coefficient }\end{array}$ & beta_sp & Normal & 42.0 & 1.0 & 0.2100 \\
\hline $\begin{array}{l}\text { Nucleate boiling heat } \\
\text { transfer coefficient }\end{array}$ & htc_nb & Normal & 24.0 & 1.0 & 0.1200 \\
\hline $\begin{array}{l}\text { Interfacial drag coeffi- } \\
\text { cient for entrainment }\end{array}$ & intfr_l & Normal & 26.0 & 1.0 & 0.1300 \\
\hline $\begin{array}{l}\text { Interfacial drag coeffi- } \\
\text { cient for entrainment }\end{array}$ & intfr_e & Normal & 34.0 & 1.0 & 0.1700 \\
\hline $\begin{array}{l}\text { Rod diameter } \\
\text { Definition }\end{array}$ & RodD & Normal & 0.02 & 1.0 & 0.007 \\
\hline Inlet coolant enthalpy & inTemp & Uniform & 1.0 & 1.0050 & 0.9950 \\
\hline
\end{tabular}

\subsubsection{PSBT Benchmark of the OECD/NEA.}

The PSBT benchmark gathers the experimental measurements of the void fraction (among other variables) at different axial levels for a rod bundle based in a PWR fuel assembly. Moreover, the experimental results count on steady-state operation conditions and transient experiments. The measure of the void fraction is done by means of the averaged measurement of the central sub-channels done by an X-Ray Densitometer. 
Development and validation of a multi-scale and multi-physics methodology for the safety analysis of fast transients in Light Water Reactors

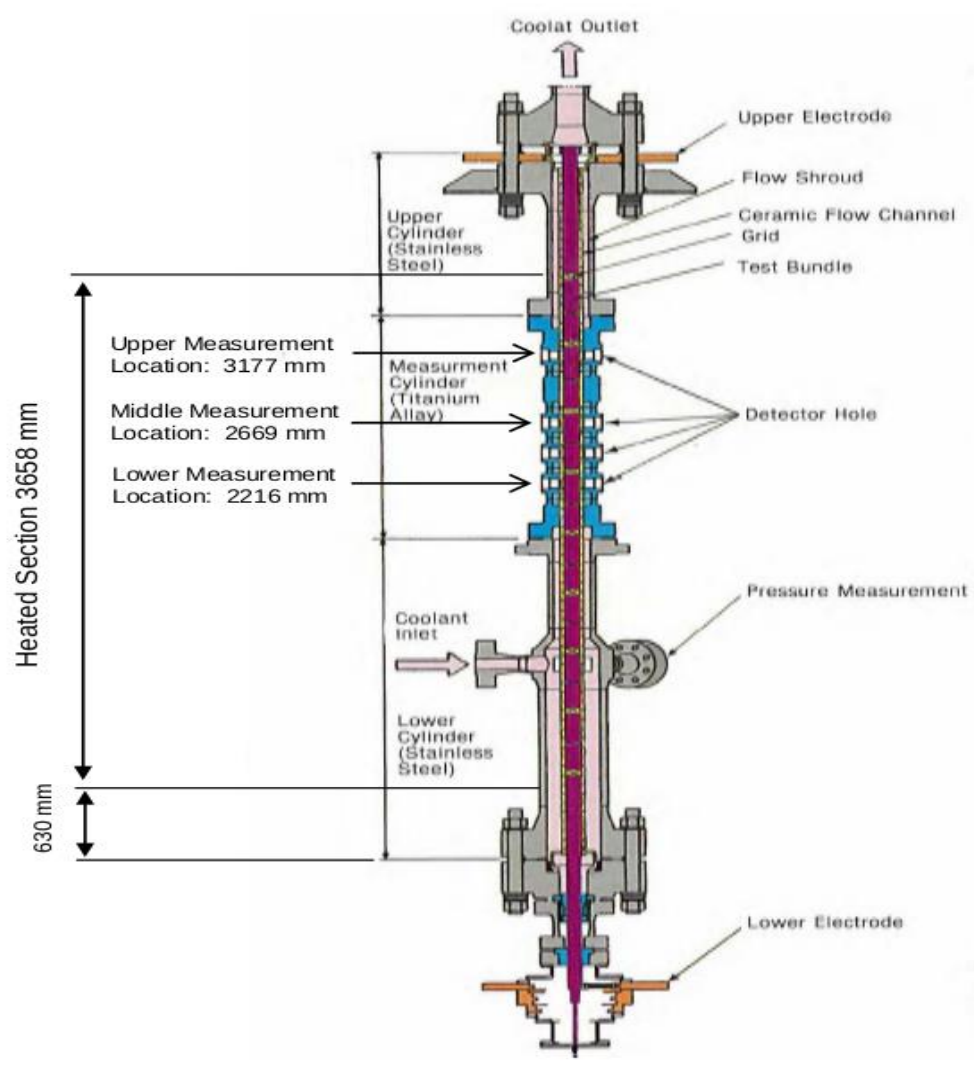

Figure 4.12 Graphical scheme of the test facility of the PSBT Benchmark (Rubin, 2010).

As Figure 4.12 shows, there are three different measure points placed at the heights of $2.216 \mathrm{~m}, 2.669 \mathrm{~m}$ and $3.177 \mathrm{~m}$, for respectively the lower, middle and upper measure points.

The PSBT benchmark include the measured values of a set of different tests, for each of the available mockup models of the experiment. The results presented in this section are based on the so-called Bundle model number 5 . The variations in the boundary conditions of power, inlet coolant temperature, outlet pressure and mass flow yield a set several test values. Among the different test results of the Benchmark, the authors will apply the BEPU methodology to a set of 11 different tests run for Bundle Type 5. These 11 test are designed varying the initial boundary conditions, namely inlet mass flow, inlet coolant temperature, bundle power and outlet pressure. The conditions of the 11 proposed 
tests are described in Table 4.7. On the other hand, the methodology is additionally applied to a transient test case of the benchmark. The transient is consisting of a Temperature Increase of the coolant at the inlet. Figure 4.13 and Figure 4.14 depict the evolution of the parameters for the transient case and shows the boundary conditions of the two scenarios. Notice that both Figure 4.13 and Figure 4.14 show up to 60 seconds within the transient since the transient starts at that time, until 120 seconds, which is the transient end time.
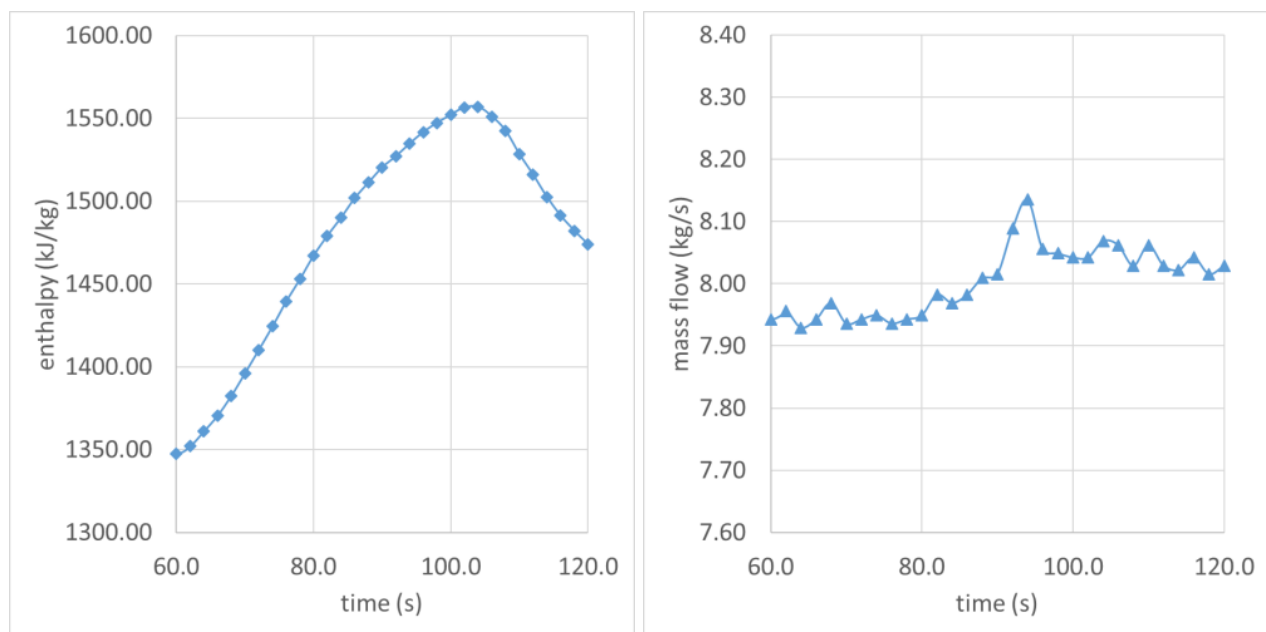

Figure 4.13 Evolution of the inlet coolant enthalpy (left) and mass flow (right).
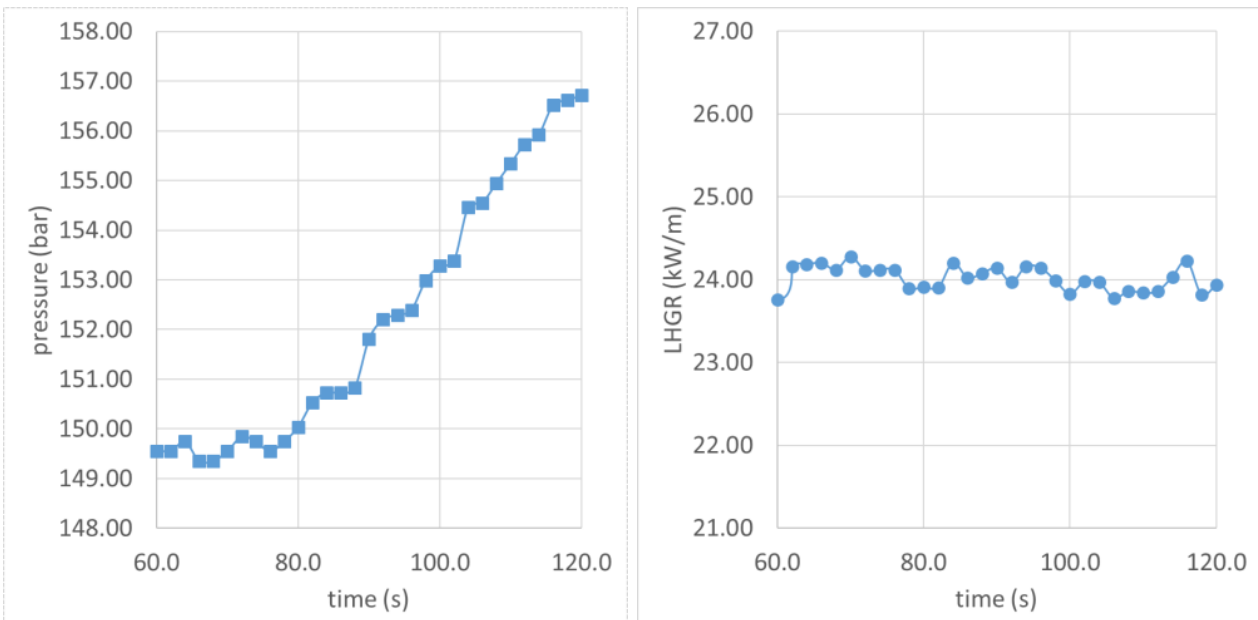

Figure 4.14 Evolution of the outlet coolant pressure (left) and the power (right). 
Development and validation of a multi-scale and multi-physics methodology for the safety analysis of fast transients in Light Water Reactors

Table 4.7 Boundary conditions for the simulation models.

\begin{tabular}{lcccc}
\hline Run No & $\begin{array}{c}\text { Pressure } \\
\left(\mathbf{k g} / \mathbf{c m}^{\mathbf{2}} \mathbf{a}\right)\end{array}$ & $\begin{array}{c}\text { Mass Flux } \\
\left(\mathbf{1 0}^{\mathbf{6}} \mathbf{k g} / \mathbf{s}^{\cdot} \mathbf{c m}^{\mathbf{2}}\right)\end{array}$ & $\begin{array}{c}\text { Power } \\
(\mathbf{k W})\end{array}$ & $\begin{array}{c}\text { Inlet Temperature } \\
\left({ }^{\mathbf{}} \mathbf{C}\right)\end{array}$ \\
\hline $\mathbf{5 . 1 2 2 1}$ & 168.29 & 11.00 & 3000 & 292.3 \\
\hline $\mathbf{5 . 1 2 2 2}$ & 168.27 & 10.98 & 2998 & 297.3 \\
\hline $\mathbf{5 . 2 1 1 1}$ & 148.15 & 15.08 & 3296 & 291.9 \\
\hline $\mathbf{5 . 2 1 1 2}$ & 148.04 & 14.98 & 3294 & 296.8 \\
\hline $\mathbf{5 . 2 4 4 2}$ & 149.97 & 4.99 & 2000 & 263.0 \\
\hline $\mathbf{5 . 3 4 4 1}$ & 125.22 & 5.00 & 2014 & 247.9 \\
\hline $\mathbf{5 . 3 4 4 2}$ & 125.13 & 5.00 & 2013 & 257.7 \\
\hline $\mathbf{5 . 4 5 6 2}$ & 100.23 & 2.02 & 1016 & 214.3 \\
\hline $\mathbf{5 . 6 3 2 1}$ & 49.2 & 7.87 & 3000 & 173.5 \\
\hline $\mathbf{5 . 6 3 2 2}$ & 49.08 & 7.86 & 3000 & 183.6 \\
\hline $\mathbf{5 . 6 5 5 2}$ & 50.17 & 2.00 & 1028 & 159.1 \\
\hline
\end{tabular}




\subsubsection{CTF-UPVIS simulation model.}

The presented BEPU methodology uses a CTF-UPVIS input deck designed in 3D. The radial distribution is done with coolant centered sub-channel distribution, i.e. the radial node is a coolant cell surrounded by four quarters of rods, or the corresponding side of the canister. The result is a radial distribution of 36 sub-channels as Figure 4.15 shows. On the other hand, the axial distribution is designed in order to fit the spacer grids with the corresponding axial height. With this distribution, the axial length counts on 35 cell nodes.

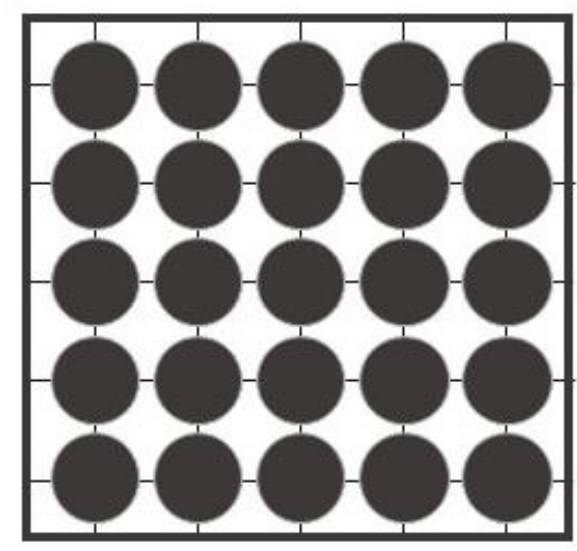

Figure 4.15 Layout of the rod array of the PSBT test facility.

The axial distribution of the nodes has been modelled in a non-uniform way. This is done to accommodate the nodal distribution to the lengths of the different spacers and to have a fine node with the location of the measurement point in the middle of the cell. Table 4.8 shows the axial nodal distribution. 
Development and validation of a multi-scale and multi-physics methodology for the safety analysis of fast transients in Light Water Reactors

Table 4.8 Nodal distribution in the axial length of the PSBT model for CTF-UPVIS.

\begin{tabular}{|c|c|c|c|c|c|c|}
\hline node (-) & 1 & 2 & 3 & 4 & 5 & 6 \\
\hline node height (m) & 0.022 & 0.139 & 0.243 & 0.360 & 0.499 & 0.612 \\
\hline node (-) & 7 & 8 & 9 & 10 & 11 & 12 \\
\hline node height (m) & 0.704 & 0.818 & 0.953 & 1.066 & 1.157 & 1.271 \\
\hline node (-) & 13 & 14 & 15 & 16 & 17 & 18 \\
\hline node height (m) & 1.406 & 1.519 & 1.611 & 1.725 & 1.860 & 1.973 \\
\hline node $(-)$ & 19 & 20 & 21 & 22 & 23 & 24 \\
\hline node height (m) & 2.065 & 2.178 & 2.313 & 2.426 & 2.518 & 2.632 \\
\hline node (-) & 25 & 26 & 27 & 28 & 29 & 30 \\
\hline node height (m) & 2.767 & 2.894 & 2.999 & 3.126 & 3.275 & 3.402 \\
\hline node $(-)$ & 31 & 32 & 33 & 34 & 35 & 36 \\
\hline node height (m) & 3.507 & 3.634 & 3.774 & 3.892 & 3.995 & - \\
\hline
\end{tabular}

The four central nodes of the radial distribution (see Figure 4.15) correspond to the location of the void measurement of the test facility. Table 4.8 has highlighted in bolds the top of the nodes where the measure points (see Figure 4.15) will be simulated, i.e. nodes 21, 24 and 28. Nevertheless, the axial distribution does not allow to use directly the prediction of the void fraction since there is no axial node fitting with the position of the measure point. Therefore, the result will be averaged between the corresponding adjacent nodes using the MATLAB (MATLAB and Statistics Toolbox Release 2017a, 2017) based application for the post-processing of the output data. For satisfying the formula of Wilks (see Equation 4.4), this exercise uses a sample of 146 cases.

The design of the nodes, gap connections among them and the geometry of heated and unheated structures is based on the geometrical parameters of the PSBT (Rubin, 2010) facility and summarized in Table 4.9. 
Chapter 4. Validation and Verification of Codes and Methodologies for two Application cases

Table 4.9 Design parameters of the test facility.

\begin{tabular}{|c|c|}
\hline Geometry parameters & Value $(\mathrm{mm})$ \\
\hline Heated rod outer diameter & 12.3 \\
\hline Heated rod pitch & 16.2 \\
\hline Water rod outer diameter & 34.0 \\
\hline Axial heated length & 3708.0 \\
\hline Flow channel inner width & 132.5 \\
\hline Flow channel corner radius & 8.0 \\
\hline Flow area & 9463.0 \\
\hline
\end{tabular}

\subsubsection{Validation results.}

This section shows the obtained results divided in two subsections for the steady state and the transient case. The subsection summarizes as well the results of the Sensitivity Analysis as a complement of the deterministic calculations completing hence the BEPU methodology. The latter shows how the uncertainty of the input parameters affect to the uncertainty of the target output variable.

Steady State results.

Figure 4.16 to Figure 4.18 shows the validation of the prediction of the void fraction of each of the three measurement points for the proposed set of 11 tests of the PSBT. The graphical results show the mean value and confidence interval of the sample compared to the experimental results. The boundaries of the distribution of the output variable are defined according to the Wilks methodology, with the sample's maximum and minimum value. With these boundaries, the reader can observe how the uncertainty of the prediction overlaps the experimental results and their error boundaries. In addition, figures include the comparison against the results of different participants of the Benchmark. The uncertainty is added to the measured void fraction according to the technical specifications of the Benchmark (Rubin, 2010). The Benchmark data defines an uncertainty of $4 \%( \pm \sigma)$ in the measurement of void fraction in steady-state.

The results of the steady-state simulations show good agreement. These results show a tendency of CTF-UPVIS to overpredict the void fraction in case of reduced values. This overprediction can be seen in

Figure 4.16 for the lower measurement point. As the void fraction increases, the predicted value shows better agreement with the measurements, as the middle and upper measurement points shown in figures Figure 4.17 Figure 4.18. Nevertheless, a clear overlapping 
Development and validation of a multi-scale and multi-physics methodology for the safety analysis of fast transients in Light Water Reactors

between the margins of the predicted and the measured value is clear, revealing a high probability of approaching the real value with CTF-UPVIS. In addition, the results of CTF-UPVIS are compared with the results of different participants, confirming the good agreement of the predictions.

The observed overprediction of the void fraction for reduced values can be related to the size of the bubbles and the possible difficulty to detect them. It is assumable that axial locations where the Onset of Nucleate Boiling (ONB) will have a smaller bubble size. Therefore, if the measurement point is close to this location, it will be more difficult to detect void, and the registered measurement is more likely to be negligible. This judgement is supported with Table 4.10 and Table 4.11 which shows the higher errors (below -0.05) for the lower measurement point, which usually are coincident with the ONB. According to CTF-UPVIS results, when the axial location of the onset of nucleate boiling is close to the measurement point, the error increases.

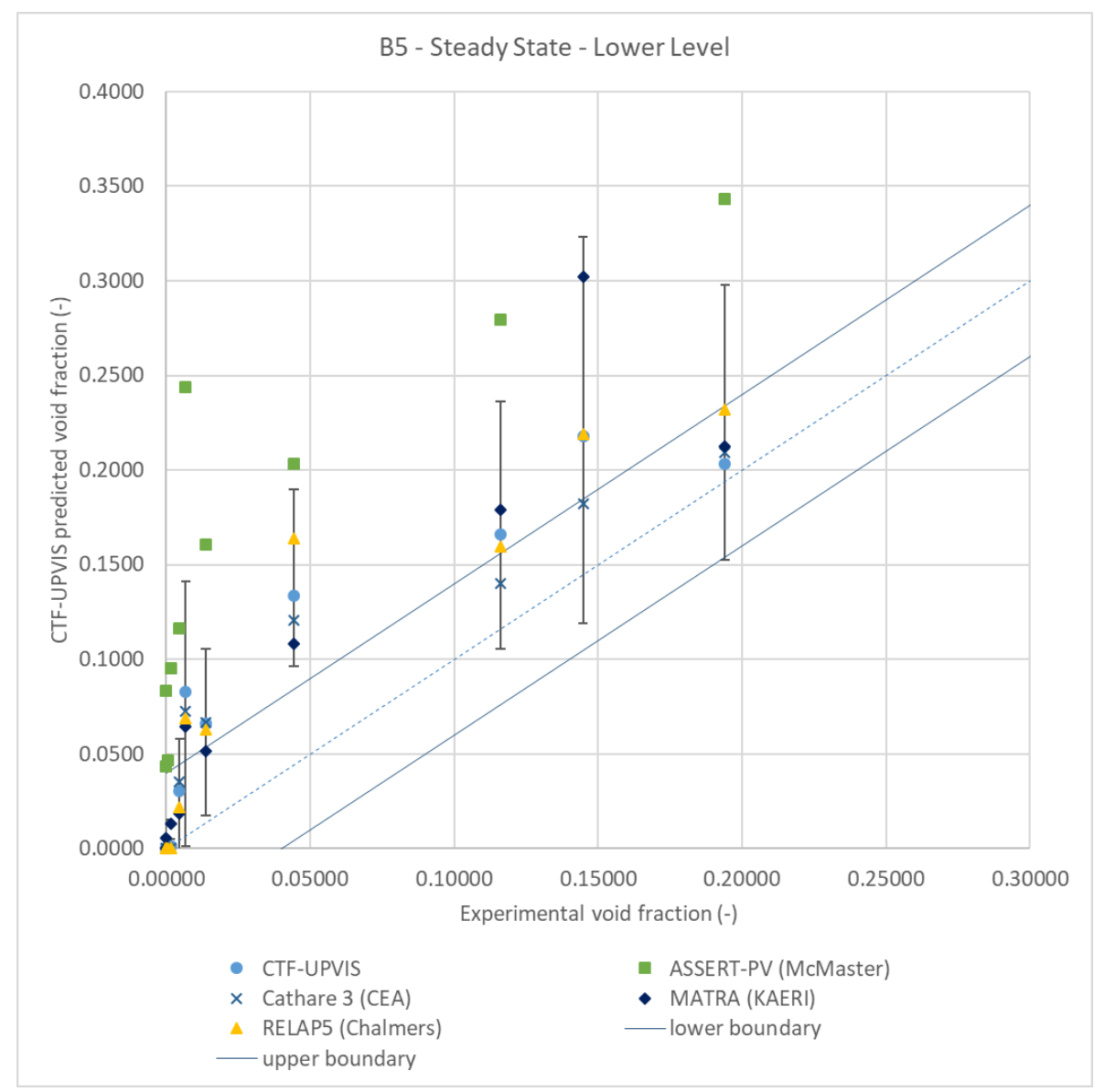


Chapter 4. Validation and Verification of Codes and Methodologies for two Application cases

Figure 4.16 Validation of the prediction of void fraction against measurement of the benchmark for the measurement point $1(2.216 \mathrm{~m})$.

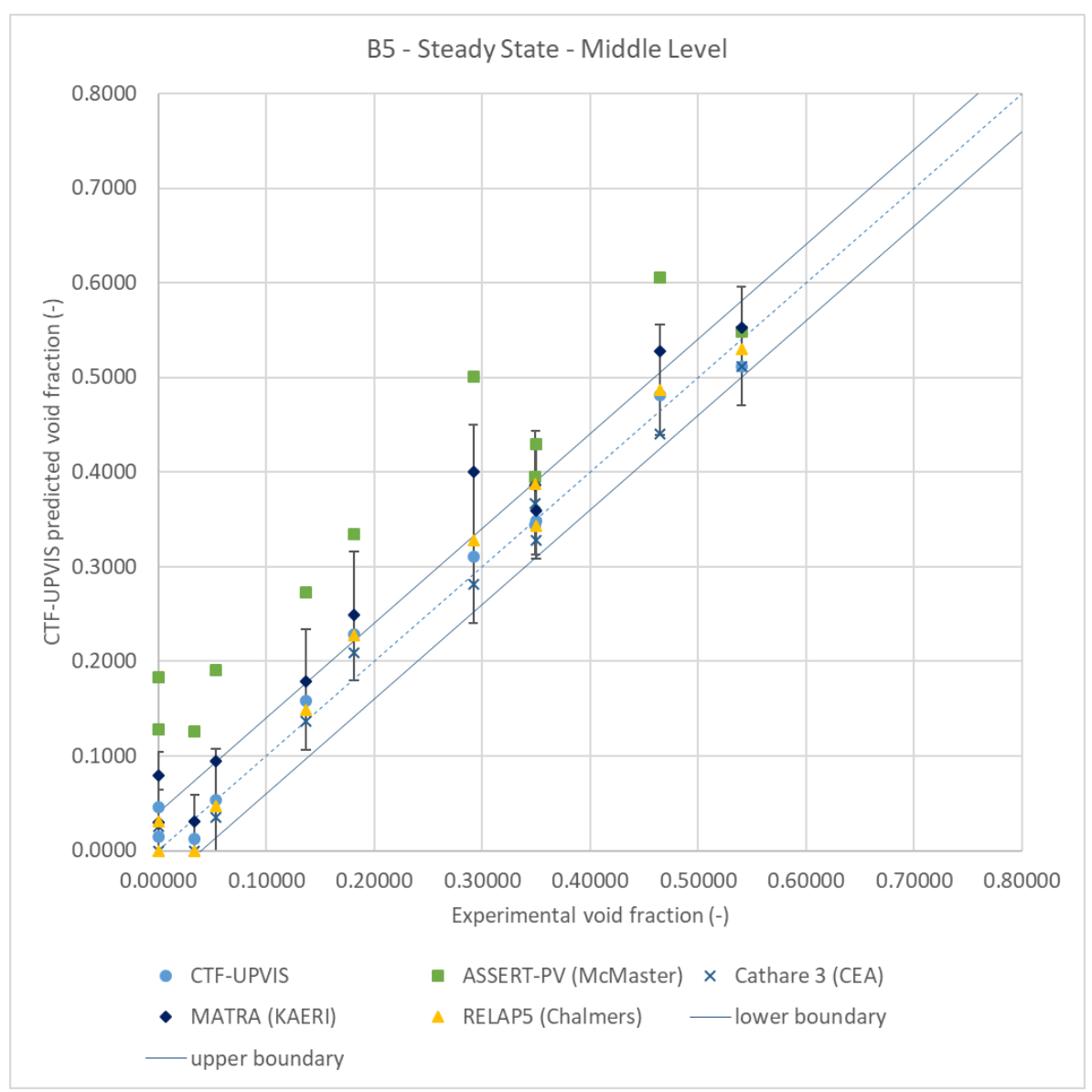

Figure 4.17 Validation of the prediction of void fraction against measurement of the benchmark for the measurement point $2(2.669 \mathrm{~m})$. 
Development and validation of a multi-scale and multi-physics methodology for the safety analysis of fast transients in Light Water Reactors

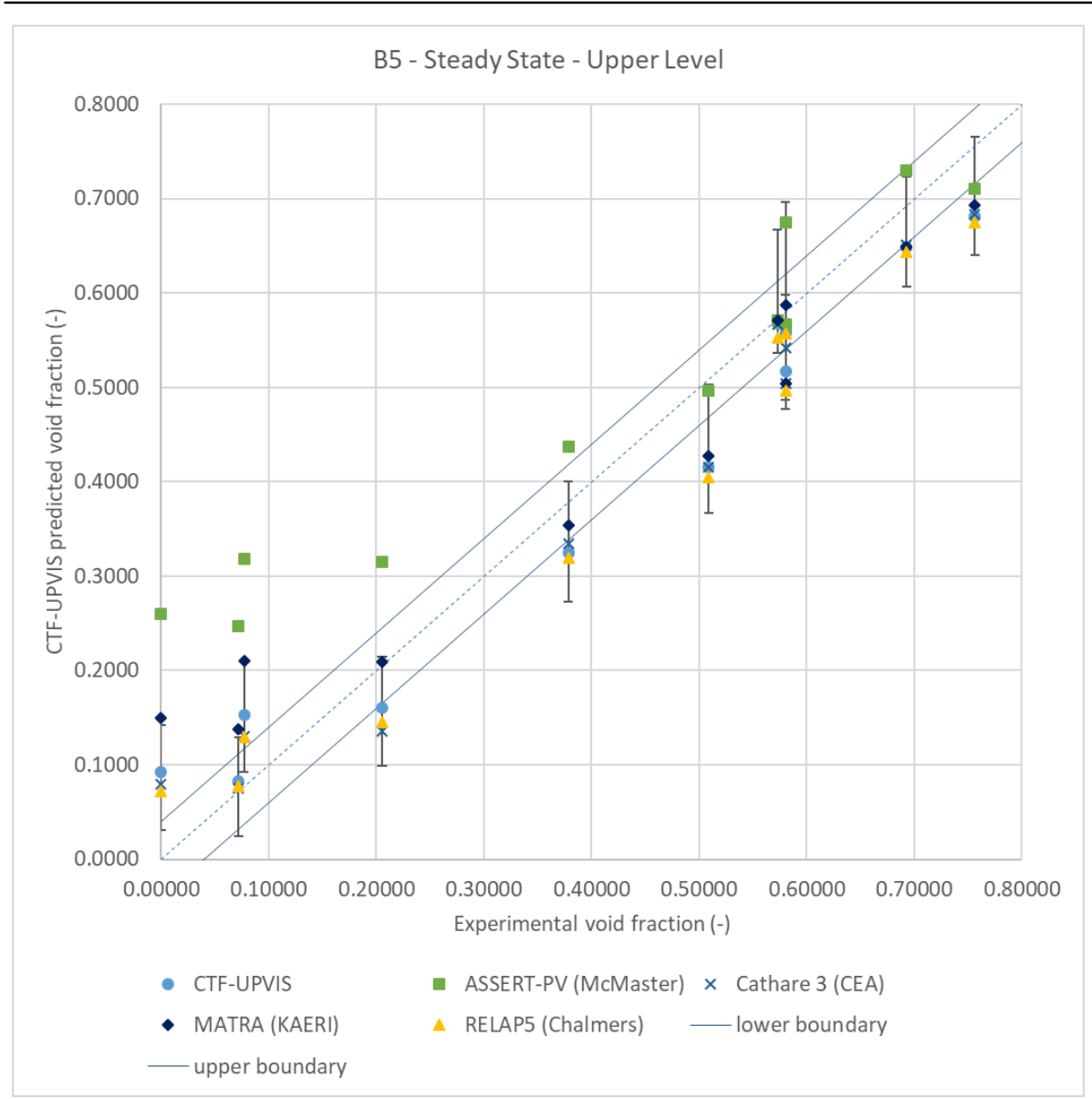

Figure 4.18 Validation of the prediction of void fraction against measurement of the benchmark for the measurement point $3(3.177 \mathrm{~m})$. 
Chapter 4. Validation and Verification of Codes and Methodologies for two Application cases

Table 4.10 Absolute error of the comparison between CTF prediction and measured value and test boundary conditions.

\begin{tabular}{|c|c|c|c|c|c|c|c|}
\hline $\begin{array}{l}\text { Run } \\
\text { No. }\end{array}$ & $\begin{array}{c}\text { Pressure } \\
\left(\mathrm{kg} / \mathrm{cm}^{2} \mathbf{a}\right)\end{array}$ & $\begin{array}{c}\text { Mass Flux } \\
\left(10^{6} \mathrm{~kg} / \mathbf{s}^{\cdot} \mathbf{c m}^{2}\right)\end{array}$ & $\begin{array}{c}\text { Power } \\
(k W)\end{array}$ & $\begin{array}{c}\text { Inlet } \\
\text { Temp. } \\
\left({ }^{\circ} \mathrm{C}\right)\end{array}$ & $\begin{array}{c}\text { CTF. } \\
\text { (Lower) }\end{array}$ & $\begin{array}{c}\text { CTF. } \\
\text { (Middle) }\end{array}$ & $\begin{array}{c}\text { CTF. } \\
\text { (Upper) }\end{array}$ \\
\hline 5.1221 & 168.29 & 11.00 & 3000.00 & 292.30 & 0.0000 & -0.0141 & -0.0920 \\
\hline 5.1222 & 168.27 & 10.98 & 2998.00 & 297.30 & 0.0000 & -0.0462 & -0.0761 \\
\hline 5.2111 & 148.15 & 15.08 & 3296.00 & 291.90 & 0.0005 & 0.0215 & -0.0105 \\
\hline 5.2112 & 148.04 & 14.98 & 3294.00 & 296.80 & 0.0002 & -0.0001 & 0.0452 \\
\hline 5.2442 & 149.97 & 4.99 & 2000.00 & 263.00 & -0.0258 & -0.0218 & 0.0532 \\
\hline 5.3441 & 125.22 & 5.00 & 2014.00 & 247.90 & -0.0524 & -0.0474 & 0.0935 \\
\hline 5.3442 & 125.13 & 5.00 & 2013.00 & 257.70 & -0.0499 & 0.0011 & 0.0638 \\
\hline 5.4562 & 100.23 & 2.02 & 1016.00 & 214.30 & -0.0895 & 0.0052 & 0.0047 \\
\hline 5.6321 & 49.20 & 7.87 & 3000.00 & 173.50 & -0.0763 & -0.0193 & 0.0225 \\
\hline 5.6322 & 49.08 & 7.86 & 3000.00 & 183.60 & -0.0729 & -0.0165 & 0.0440 \\
\hline 5.6552 & 50.17 & 2.00 & 1028.00 & 159.10 & -0.0090 & 0.0288 & 0.0754 \\
\hline
\end{tabular}

Table 4.11 Absolute error of the comparison between CTF prediction and measured value and location of ONB.

\begin{tabular}{|c|c|c|c|c|c|c|}
\hline $\begin{array}{l}\text { Run } \\
\text { No. }\end{array}$ & $\begin{array}{c}\text { CTF. } \\
\text { (Lower) }\end{array}$ & $\begin{array}{c}\text { CTF. } \\
\text { (Middle) }\end{array}$ & $\begin{array}{c}\text { CTF. } \\
\text { (Upper) }\end{array}$ & $\begin{array}{c}\text { Axial } \\
\text { node for } \\
\text { ONB }\end{array}$ & $\begin{array}{c}\text { Elevation } \\
\text { of ONB } \\
\text { (m) }\end{array}$ & $\begin{array}{l}\text { Axial location of ONB rela- } \\
\text { tive to measurement points }\end{array}$ \\
\hline 5.1221 & 0.0000 & -0.0141 & -0.0920 & 32 & 3.501 & Upper $<$ ONB \\
\hline 5.1222 & 0.0000 & -0.0462 & -0.0761 & 30 & 3.247 & Upper $<$ ONB \\
\hline 5.2111 & 0.0005 & 0.0215 & -0.0105 & 32 & 3.501 & Upper $<$ ONB \\
\hline 5.2112 & 0.0002 & -0.0001 & 0.0452 & 29 & 3.006 & Middle $<$ ONB $<$ Upper \\
\hline 5.2442 & -0.0258 & -0.0218 & 0.0532 & 26 & 2.739 & Middle $<$ ONB $<$ Upper \\
\hline 5.3441 & -0.0524 & -0.0474 & 0.0935 & 24 & 2.512 & Lower $<$ ONB $<$ Middle \\
\hline 5.3442 & -0.0499 & 0.0011 & 0.0638 & 22 & 2.285 & Lower $<$ ONB $<$ Middle \\
\hline 5.4562 & -0.0895 & 0.0052 & 0.0047 & 22 & 2.285 & Lower $<$ ONB $<$ Middle \\
\hline 5.6321 & -0.0763 & -0.0193 & 0.0225 & 23 & 2.341 & ONB $<$ Lower \\
\hline 5.6322 & -0.0729 & -0.0165 & 0.0440 & 21 & 2.072 & ONB $<$ Lower \\
\hline 5.6552 & -0.0090 & 0.0288 & 0.0754 & 21 & 2.072 & ONB $<$ Lower \\
\hline
\end{tabular}


Development and validation of a multi-scale and multi-physics methodology for the safety analysis of fast transients in Light Water Reactors

Sensitivity Analysis of the steady-state results.

The U\&S analysis provides additional support to explain the behavior of the prediction of the void fraction regarding the variation of the input parameters or boundary conditions. More specifically, the Sensitivity Analysis defines the correlation of the input variables that have been perturbed for generating the samples with the void fraction predicted by CTF-UPVIS. 
Table 4.12 Sensitivity Analysis of Test Run 5.1121. show the PRCC of the perturbed input variables with regard to the predicted different void fraction of the CTF-UPVIS model. The correlation of one input parameter is considered to be relevant up to values of \pm 0 .2. Figures Figure 4.19 to Figure 4.29 support graphically the results of the SA for each test.

In view of the results, the boundary conditions as well as the rod geometry play a relevant role in the prediction of void fraction. Nevertheless, no general conclusions can be developed in this regard, since the experiment varies simultaneously all parameters in both directions for the different tests, instead of varying one single parameter at a time to observe its influence in the prediction error. The highest PRCCs are observed for the rod diameter. This is so, because the size of the diameter is directly related to the crosssectional mass flux, and the void generation is analytically related to this variable. This is also confirmed with the PRCC of the mass flow. Bundle Power and Inlet Coolant temperature have a positive PRCC and therefore, an increase in such variables would lead to an increase in void fraction prediction. Conversely, Inlet Mass Flux and Outlet Pressure have negative PRCCs and show the higher values compared to the other two input parameters. This fact is confirmed in 
Development and validation of a multi-scale and multi-physics methodology for the safety analysis of fast transients in Light Water Reactors

Table 4.10, where it can be observed that lower pressure and mass flux values show the higher overprediction. 
Chapter 4. Validation and Verification of Codes and Methodologies for two Application cases

Table 4.12 Sensitivity Analysis of Test Run 5.1121.

\begin{tabular}{|l|l|r|r|r|}
\cline { 3 - 5 } \multicolumn{2}{c}{} & \multicolumn{3}{c|}{ CTF-UPVIS Void Fraction } \\
\hline Input Variable & Label & \multicolumn{1}{c|}{ Lower } & Middle & Upper \\
\hline LHGR & lhgr & 0.38 & 0.40 & 0.40 \\
\hline Outlet Pressure & outP & -0.73 & -0.74 & -0.74 \\
\hline Inlet Mass Flow & mflow & -0.56 & -0.57 & -0.61 \\
\hline Eq. distribution weighting factor for void drift & aaak & 0.05 & 0.08 & 0.07 \\
\hline 2-phase multiplier of the mixing coefficient & thetm & -0.09 & -0.11 & -0.20 \\
\hline 1-phase mixing coefficient & beta_sp & -0.60 & -0.70 & -0.76 \\
\hline Nucleate boiling heat transfer coefficient & htc_nb & 0.05 & 0.07 & 0.05 \\
\hline Interfacial drag coefficient for liquid & intfr_l & 0.03 & 0.60 & 0.69 \\
\hline Interfacial drag coefficient for entrainment & intfr_e & -0.05 & -0.04 & -0.03 \\
\hline Rod diameter & drod & -0.96 & -0.95 & -0.92 \\
\hline Inlet coolant enthalpy & inEnth & 0.91 & 0.91 & 0.91 \\
\hline
\end{tabular}

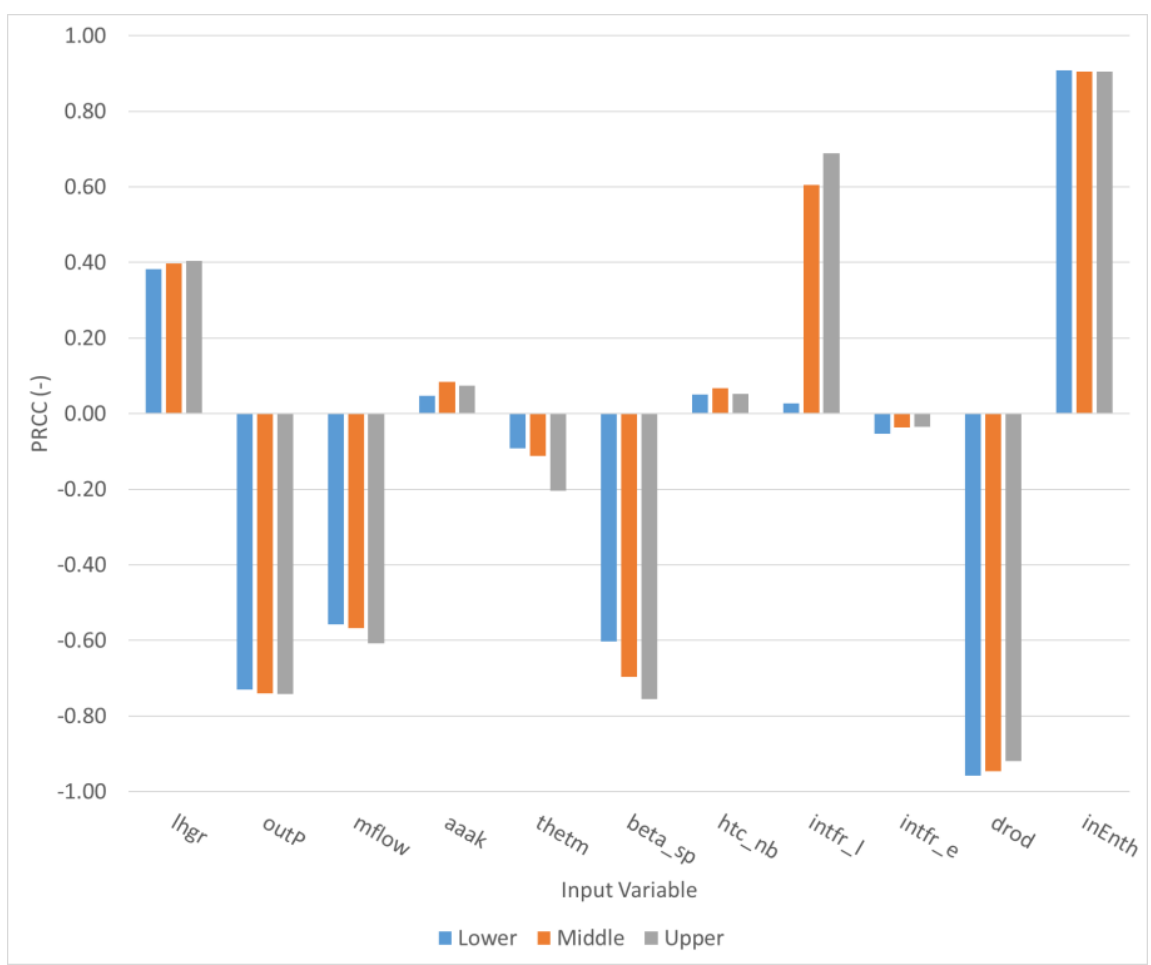

Figure 4.19 Diagram of the PRCCs for Test Run 5.1121.

Table 4.13 Sensitivity Analysis of Test Run 5.1222. 
Development and validation of a multi-scale and multi-physics methodology for the safety analysis of fast transients in Light Water Reactors

\begin{tabular}{|l|l|r|r|r|}
\cline { 3 - 5 } \multicolumn{2}{c}{} & \multicolumn{3}{c|}{ CTF-UPVIS Void Fraction } \\
\hline Input Variable & Label & \multicolumn{1}{c|}{ Lower } & Middle & Upper \\
\hline LHGR & lhgr & 0.20 & 0.50 & 0.45 \\
\hline Outlet Pressure & outP & -0.16 & -0.70 & -0.66 \\
\hline Inlet Mass Flow & mflow & -0.34 & -0.66 & -0.63 \\
\hline Eq. distribution weighting factor for void drift & aaak & -0.02 & 0.05 & 0.03 \\
\hline 2-phase multiplier of the mixing coefficient & thetm & 0.12 & -0.11 & -0.17 \\
\hline 1-phase mixing coefficient & beta_sp & -0.34 & -0.72 & -0.77 \\
\hline Nucleate boiling heat transfer coefficient & htc_nb & -0.11 & 0.04 & 0.06 \\
\hline Interfacial drag coefficient for liquid & intfr_I & 0.06 & 0.40 & 0.75 \\
\hline Interfacial drag coefficient for entrainment & intfr_e & 0.08 & -0.05 & -0.06 \\
\hline Rod diameter & drod & -0.83 & -0.96 & -0.93 \\
\hline Inlet coolant enthalpy & inEnth & 0.54 & 0.88 & 0.86 \\
\hline
\end{tabular}

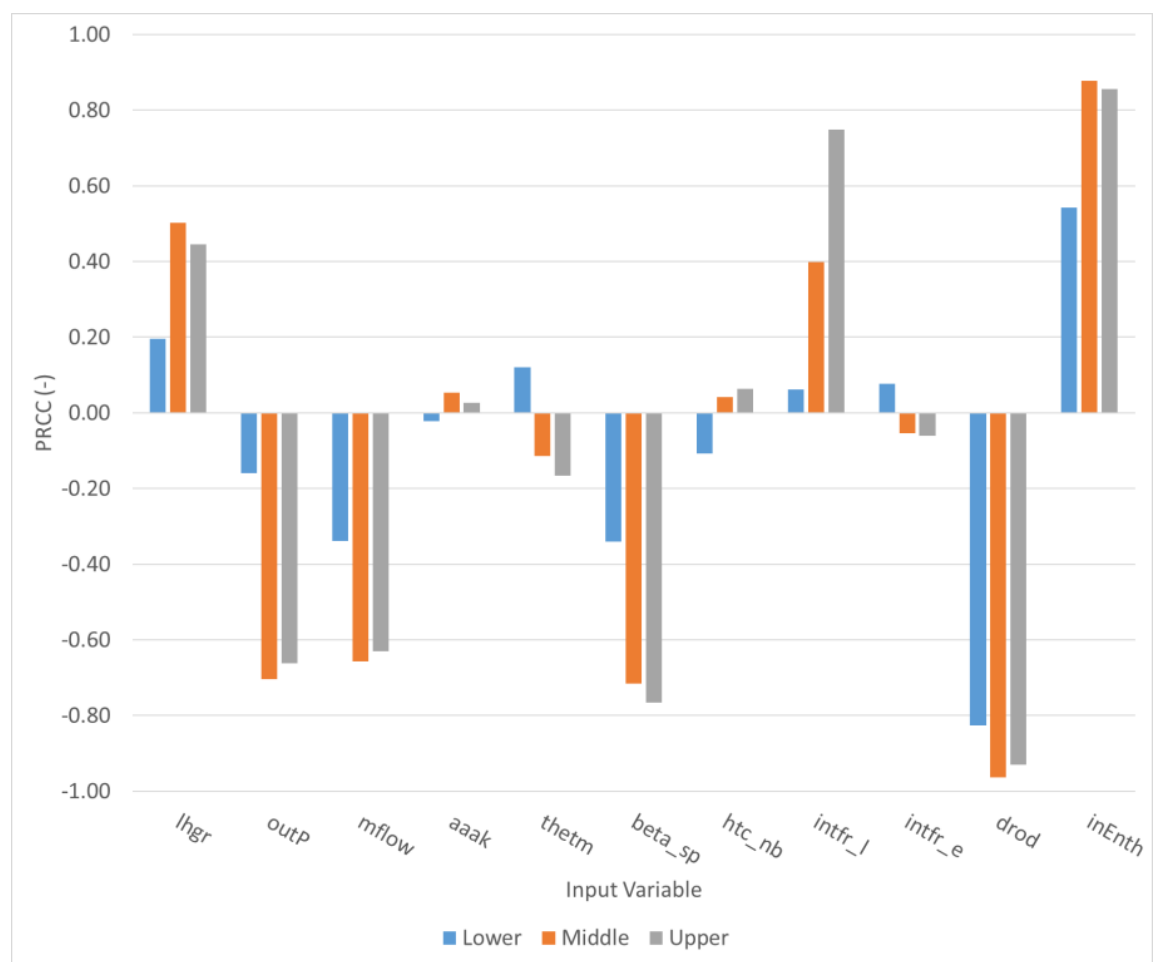

Figure 4.20 Diagram of the PRCCs for Test Run 5.1122. 
Chapter 4. Validation and Verification of Codes and Methodologies for two Application cases

Table 4.14 Sensitivity Analysis of Test Run 5.2111.

\begin{tabular}{|l|l|r|r|r|}
\cline { 3 - 5 } \multicolumn{2}{c}{} & \multicolumn{3}{c|}{ CTF-UPVIS Void Fraction } \\
\hline Input Variable & Label & \multicolumn{1}{c|}{ Lower } & Middle & Upper \\
\hline LHGR & lhgr & $*$ & 0.48 & 0.48 \\
\hline Outlet Pressure & outP & $*$ & -0.68 & -0.69 \\
\hline Inlet Mass Flow & mflow & $*$ & -0.64 & -0.62 \\
\hline Eq. distribution weighting factor for void drift & aaak & $*$ & 0.07 & 0.06 \\
\hline 2-phase multiplier of the mixing coefficient & thetm & $*$ & -0.12 & -0.13 \\
\hline 1-phase mixing coefficient & beta_sp & $*$ & -0.68 & -0.74 \\
\hline Nucleate boiling heat transfer coefficient & htc_nb & $*$ & 0.04 & 0.07 \\
\hline Interfacial drag coefficient for liquid & intfr_l & $*$ & 0.02 & 0.59 \\
\hline Interfacial drag coefficient for entrainment & intfr_e & $*$ & -0.06 & -0.04 \\
\hline Rod diameter & drod & $*$ & -0.96 & -0.95 \\
\hline Inlet coolant enthalpy & inEnth & $*$ & 0.89 & 0.88 \\
\hline
\end{tabular}

* Void prediction is 0.0 and therefore PRCC does not show a real number.

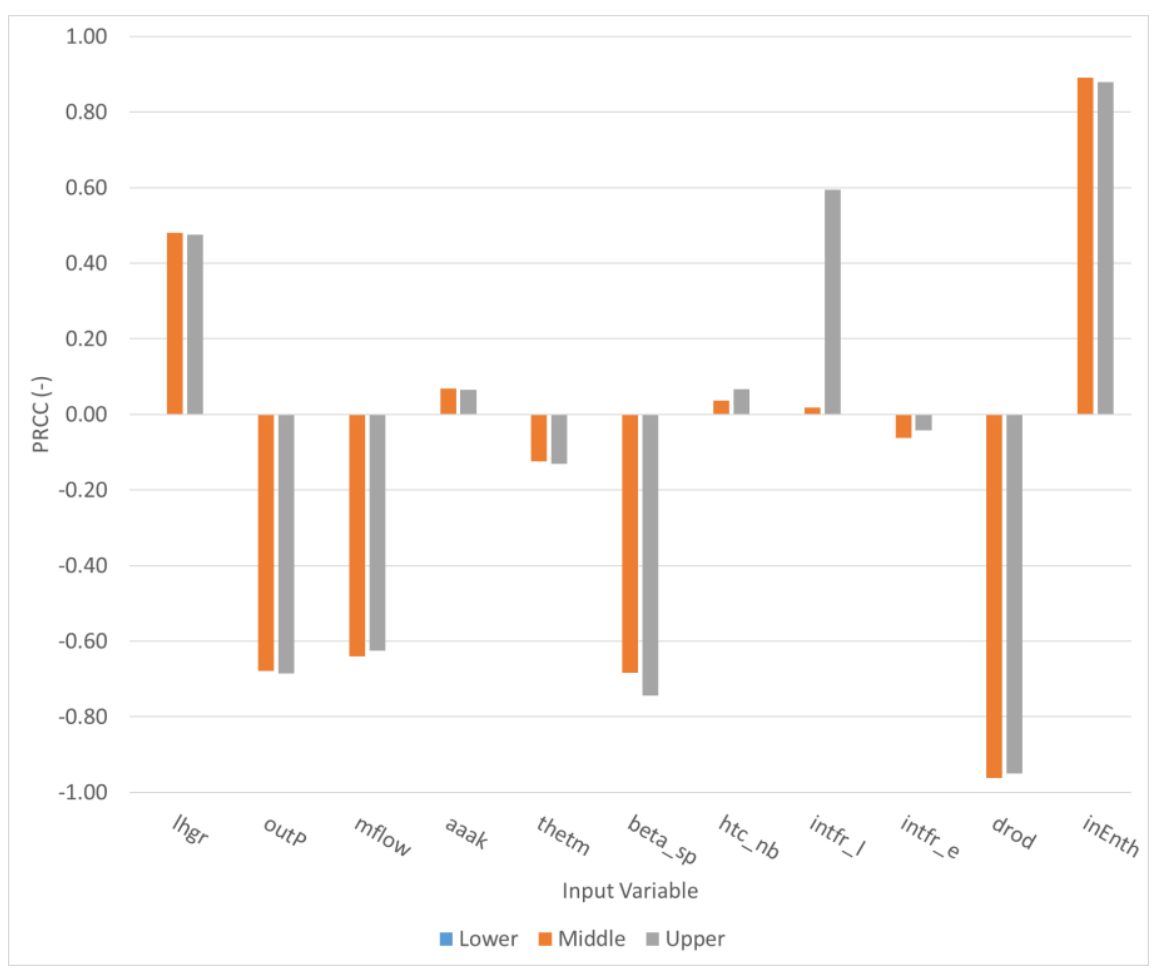

Figure 4.21 Diagram of the PRCCs for Test Run 5.2111. 
Development and validation of a multi-scale and multi-physics methodology for the safety analysis of fast transients in Light Water Reactors

Table 4.15 Sensitivity Analysis of Test Run 5.2112.

\begin{tabular}{|l|l|r|r|r|}
\cline { 3 - 5 } \multicolumn{2}{c}{} & \multicolumn{3}{c|}{ CTF-UPVIS Void Fraction } \\
\hline Input Variable & Label & \multicolumn{1}{c|}{ Lower } & Middle & Upper \\
\hline LHGR & lhgr & 0.28 & 0.47 & 0.44 \\
\hline Outlet Pressure & outP & -0.46 & -0.69 & -0.70 \\
\hline Inlet Mass Flow & mflow & -0.48 & -0.62 & -0.64 \\
\hline Eq. distribution weighting factor for void drift & aaak & 0.03 & 0.07 & 0.03 \\
\hline 2-phase multiplier of the mixing coefficient & thetm & -0.04 & -0.11 & -0.15 \\
\hline 1-phase mixing coefficient & beta_sp & -0.44 & -0.69 & -0.77 \\
\hline Nucleate boiling heat transfer coefficient & htc_nb & -0.10 & 0.02 & 0.04 \\
\hline Interfacial drag coefficient for liquid & intfr_I & 0.10 & 0.37 & 0.72 \\
\hline Interfacial drag coefficient for entrainment & intfr_e & -0.04 & -0.05 & -0.06 \\
\hline Rod diameter & drod & -0.92 & -0.96 & -0.94 \\
\hline Inlet coolant enthalpy & inEnth & 0.91 & 0.91 & 0.91 \\
\hline
\end{tabular}

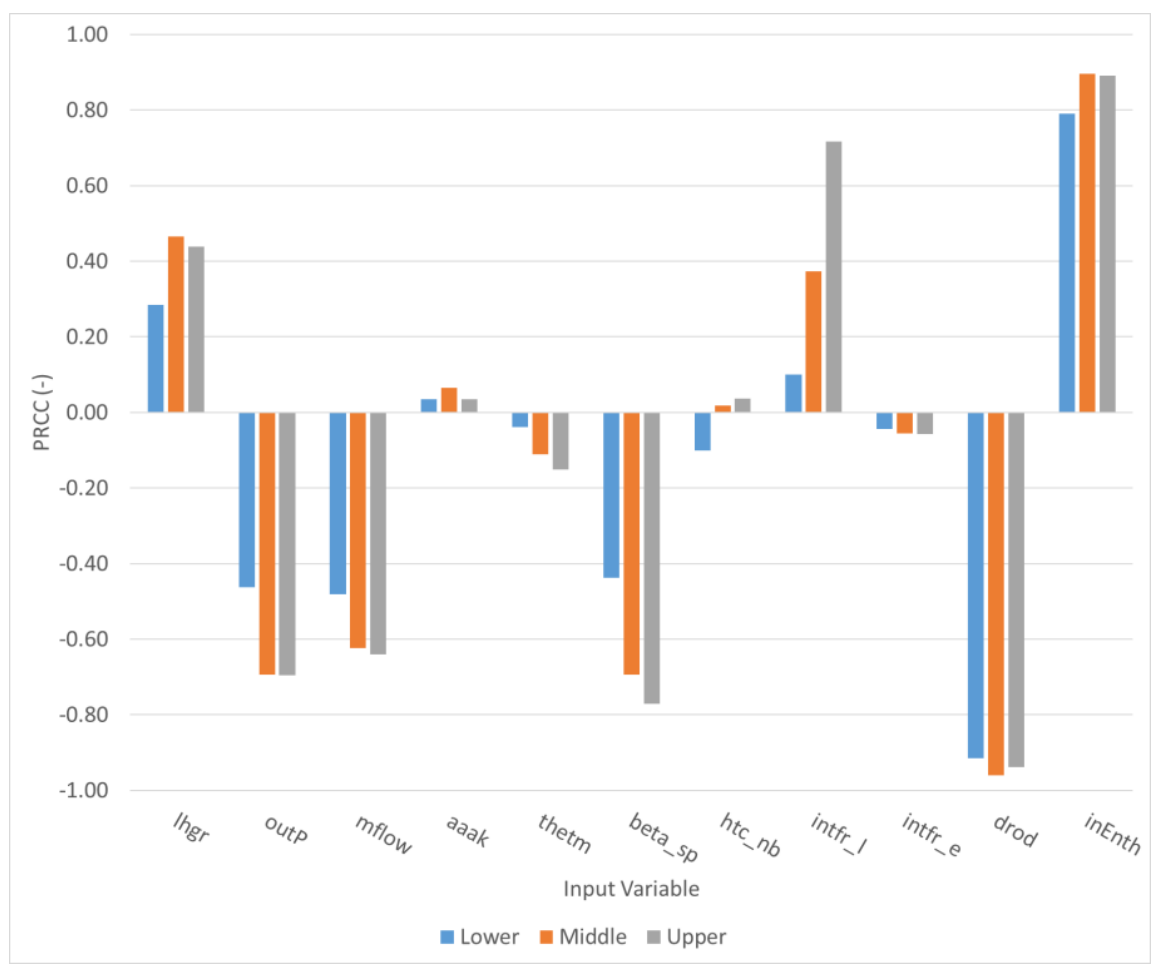

Figure 4.22 Diagram of the PRCCs for Test Run 5.2112. 
Chapter 4. Validation and Verification of Codes and Methodologies for two Application cases

Table 4.16 Sensitivity Analysis of Test Run 5.2442.

\begin{tabular}{|l|l|r|r|r|}
\cline { 3 - 5 } \multicolumn{2}{c}{} & \multicolumn{3}{c|}{ CTF-UPVIS Void Fraction } \\
\hline Input Variable & Label & \multicolumn{1}{c|}{ Lower } & Middle & Upper \\
\hline LHGR & lhgr & 0.60 & 0.45 & 0.51 \\
\hline Outlet Pressure & outP & -0.65 & -0.52 & -0.58 \\
\hline Inlet Mass Flow & mflow & -0.71 & -0.58 & -0.68 \\
\hline Eq. distribution weighting factor for void drift & aaak & 0.09 & 0.02 & 0.00 \\
\hline 2-phase multiplier of the mixing coefficient & thetm & -0.15 & -0.10 & -0.27 \\
\hline 1-phase mixing coefficient & beta_sp & -0.69 & -0.68 & -0.79 \\
\hline Nucleate boiling heat transfer coefficient & htc_nb & 0.03 & 0.03 & 0.01 \\
\hline Interfacial drag coefficient for liquid & intfr_l & 0.36 & 0.83 & 0.86 \\
\hline Interfacial drag coefficient for entrainment & intfr_e & -0.02 & -0.04 & -0.05 \\
\hline Rod diameter & drod & -0.97 & -0.92 & -0.88 \\
\hline Inlet coolant enthalpy & inEnth & 0.84 & 0.74 & 0.81 \\
\hline
\end{tabular}

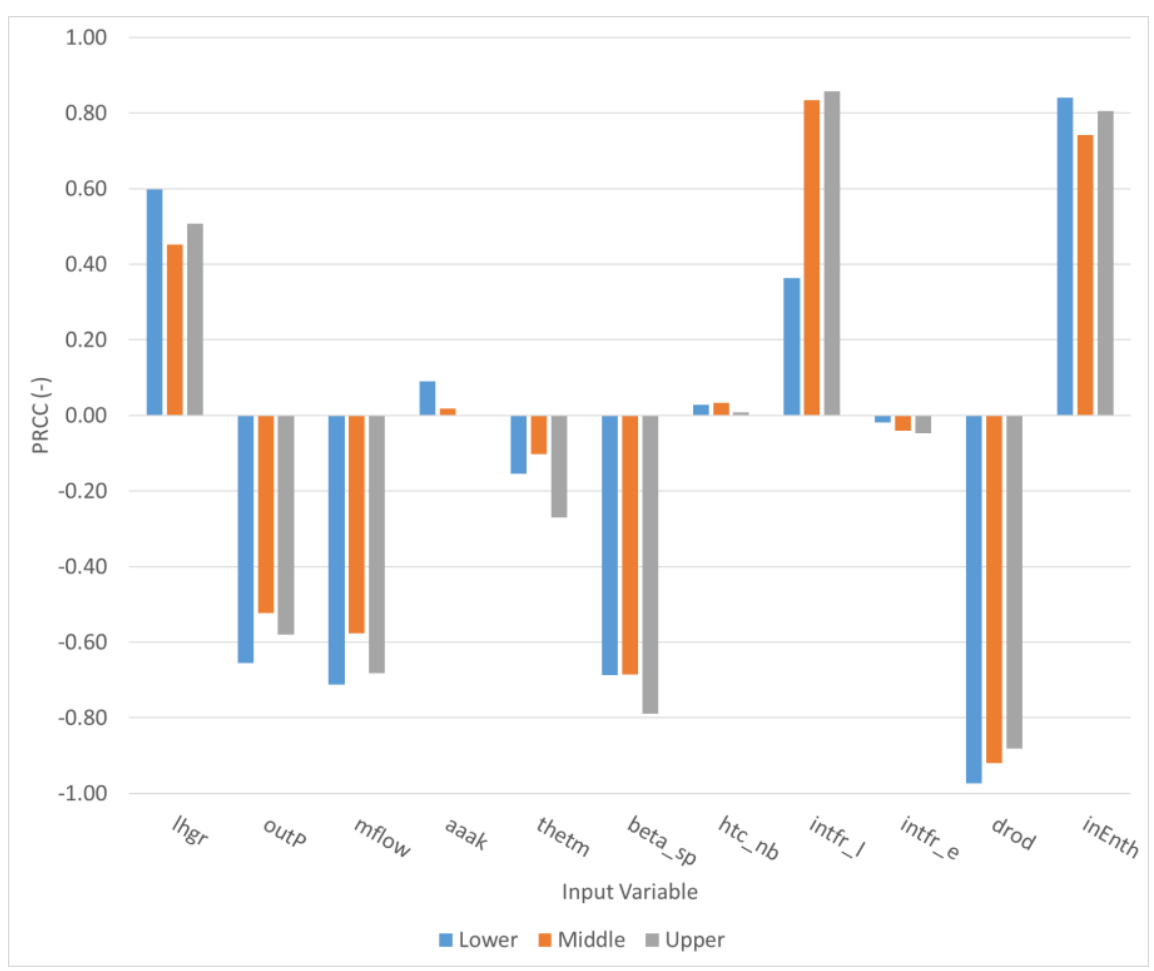

Figure 4.23 Diagram of the PRCCs for Test Run 5.2442. 
Development and validation of a multi-scale and multi-physics methodology for the safety analysis of fast transients in Light Water Reactors

Table 4.17 Sensitivity Analysis of Test Run 5.3441.

\begin{tabular}{|l|l|r|r|r|}
\cline { 3 - 5 } \multicolumn{2}{c}{} & \multicolumn{3}{c|}{ CTF-UPVIS Void Fraction } \\
\hline Input Variable & Label & \multicolumn{1}{c|}{ Lower } & Middle & Upper \\
\hline LHGR & lhgr & 0.57 & 0.43 & 0.49 \\
\hline Outlet Pressure & outP & -0.59 & -0.47 & -0.54 \\
\hline Inlet Mass Flow & mflow & -0.69 & -0.58 & -0.68 \\
\hline Eq. distribution weighting factor for void drift & aaak & 0.05 & 0.02 & 0.01 \\
\hline 2-phase multiplier of the mixing coefficient & thetm & -0.13 & -0.11 & -0.30 \\
\hline 1-phase mixing coefficient & beta_sp & -0.68 & -0.72 & -0.79 \\
\hline Nucleate boiling heat transfer coefficient & htc_nb & 0.04 & 0.02 & -0.03 \\
\hline Interfacial drag coefficient for liquid & intfr_I & 0.66 & 0.87 & 0.88 \\
\hline Interfacial drag coefficient for entrainment & intfr_e & -0.04 & -0.04 & -0.02 \\
\hline Rod diameter & drod & -0.97 & -0.90 & -0.85 \\
\hline Inlet coolant enthalpy & inEnth & 0.82 & 0.73 & 0.79 \\
\hline
\end{tabular}

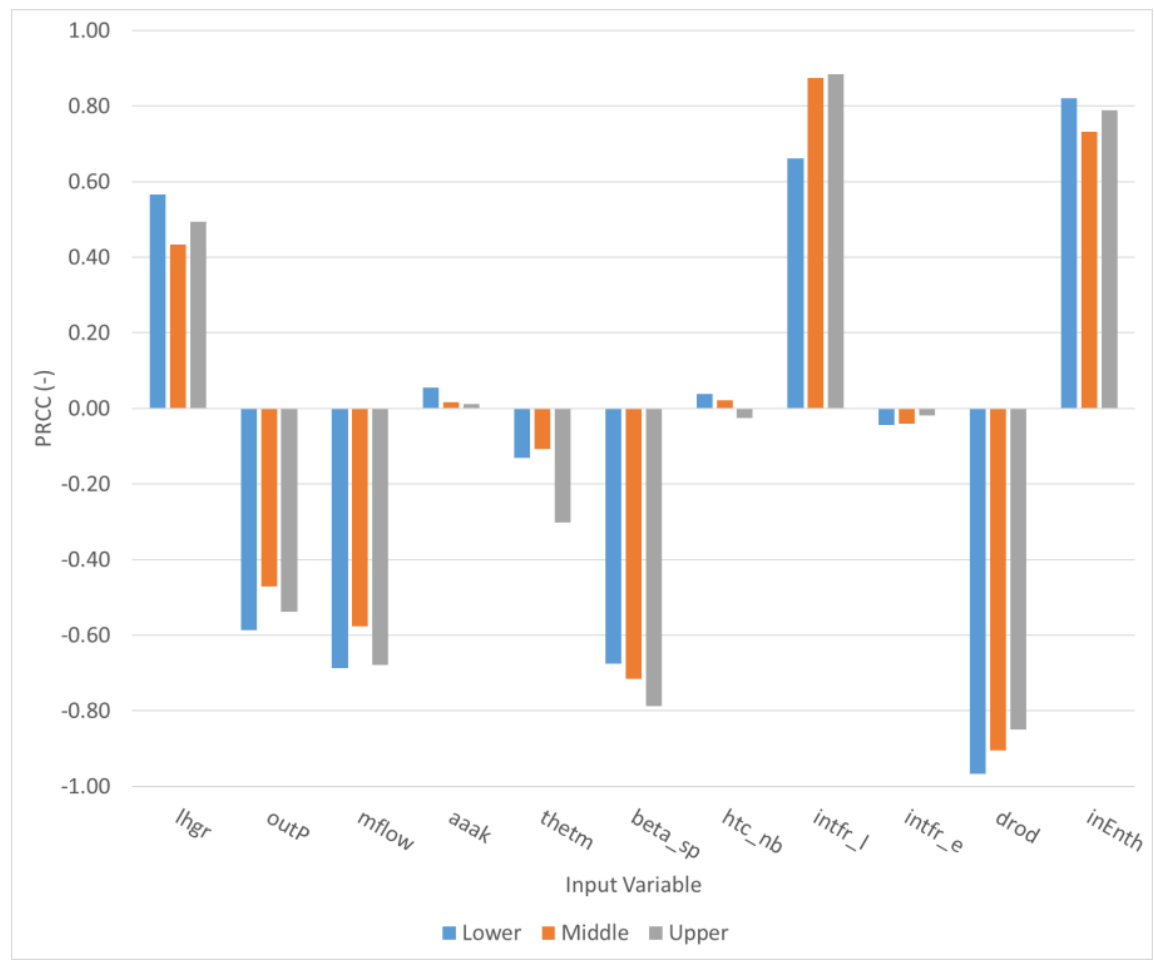

Figure 4.24 Diagram of the PRCCs for Test Run 5.3441. 
Chapter 4. Validation and Verification of Codes and Methodologies for two Application cases

Table 4.18 Sensitivity Analysis of Test Run 5.3442.

\begin{tabular}{|l|l|r|r|r|}
\cline { 3 - 5 } \multicolumn{2}{c}{} & \multicolumn{3}{c|}{ CTF-UPVIS Void Fraction } \\
\hline Input Variable & Label & \multicolumn{1}{c|}{ Lower } & Middle & Upper \\
\hline LHGR & lhgr & 0.40 & 0.42 & 0.40 \\
\hline Outlet Pressure & outP & -0.50 & -0.51 & -0.51 \\
\hline Inlet Mass Flow & mflow & -0.54 & -0.60 & -0.65 \\
\hline Eq. distribution weighting factor for void drift & aaak & 0.03 & 0.00 & 0.01 \\
\hline 2-phase multiplier of the mixing coefficient & thetm & -0.09 & -0.20 & -0.30 \\
\hline 1-phase mixing coefficient & beta_sp & -0.64 & -0.74 & -0.74 \\
\hline Nucleate boiling heat transfer coefficient & htc_nb & 0.03 & 0.01 & -0.05 \\
\hline Interfacial drag coefficient for liquid & intfr_l & 0.86 & 0.88 & 0.91 \\
\hline Interfacial drag coefficient for entrainment & intfr_e & -0.02 & -0.01 & 0.05 \\
\hline Rod diameter & drod & -0.92 & -0.87 & -0.76 \\
\hline Inlet coolant enthalpy & inEnth & 0.75 & 0.77 & 0.77 \\
\hline
\end{tabular}

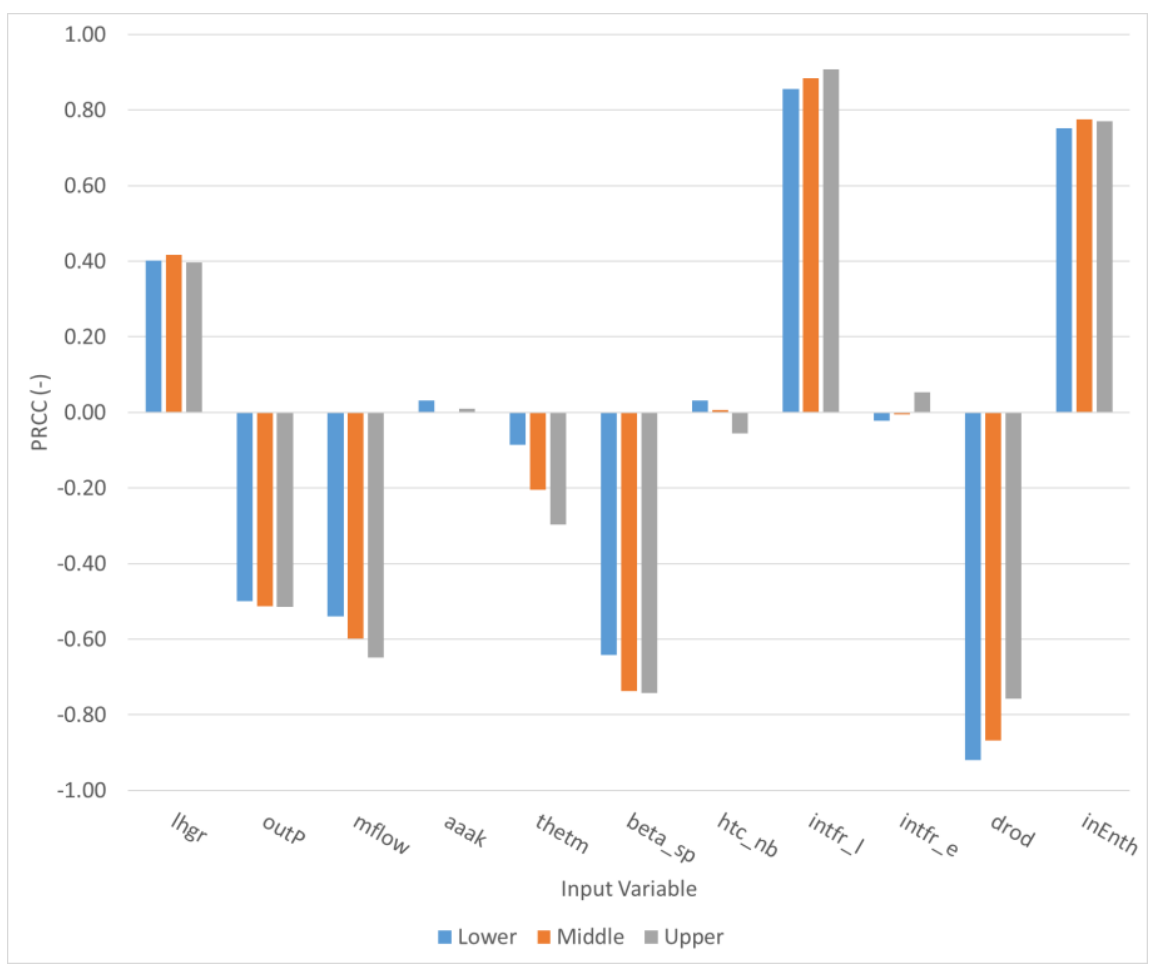

Figure 4.25 Diagram of the PRCCs for Test Run 5.3442. 
Development and validation of a multi-scale and multi-physics methodology for the safety analysis of fast transients in Light Water Reactors

Table 4.19 Sensitivity Analysis of Test Run 5.4562.

\begin{tabular}{|l|l|r|r|r|}
\cline { 3 - 5 } \multicolumn{2}{c}{} & \multicolumn{3}{c|}{ CTF-UPVIS Void Fraction } \\
\hline Input Variable & Label & \multicolumn{1}{c|}{ Lower } & Middle & Upper \\
\hline LHGR & lhgr & 0.51 & 0.63 & 0.61 \\
\hline Outlet Pressure & outP & -0.52 & -0.65 & -0.60 \\
\hline Inlet Mass Flow & mflow & -0.65 & -0.80 & -0.81 \\
\hline Eq. distribution weighting factor for void drift & aaak & 0.06 & 0.03 & 0.11 \\
\hline 2-phase multiplier of the mixing coefficient & thetm & 0.01 & 0.06 & 0.14 \\
\hline 1-phase mixing coefficient & beta_sp & -0.37 & -0.45 & -0.38 \\
\hline Nucleate boiling heat transfer coefficient & htc_nb & 0.01 & -0.05 & -0.09 \\
\hline Interfacial drag coefficient for liquid & intfr_I & 0.92 & 0.96 & 0.97 \\
\hline Interfacial drag coefficient for entrainment & intfr_e & -0.01 & 0.02 & 0.12 \\
\hline Rod diameter & drod & -0.86 & -0.49 & -0.45 \\
\hline Inlet coolant enthalpy & inEnth & 0.74 & 0.83 & 0.80 \\
\hline
\end{tabular}

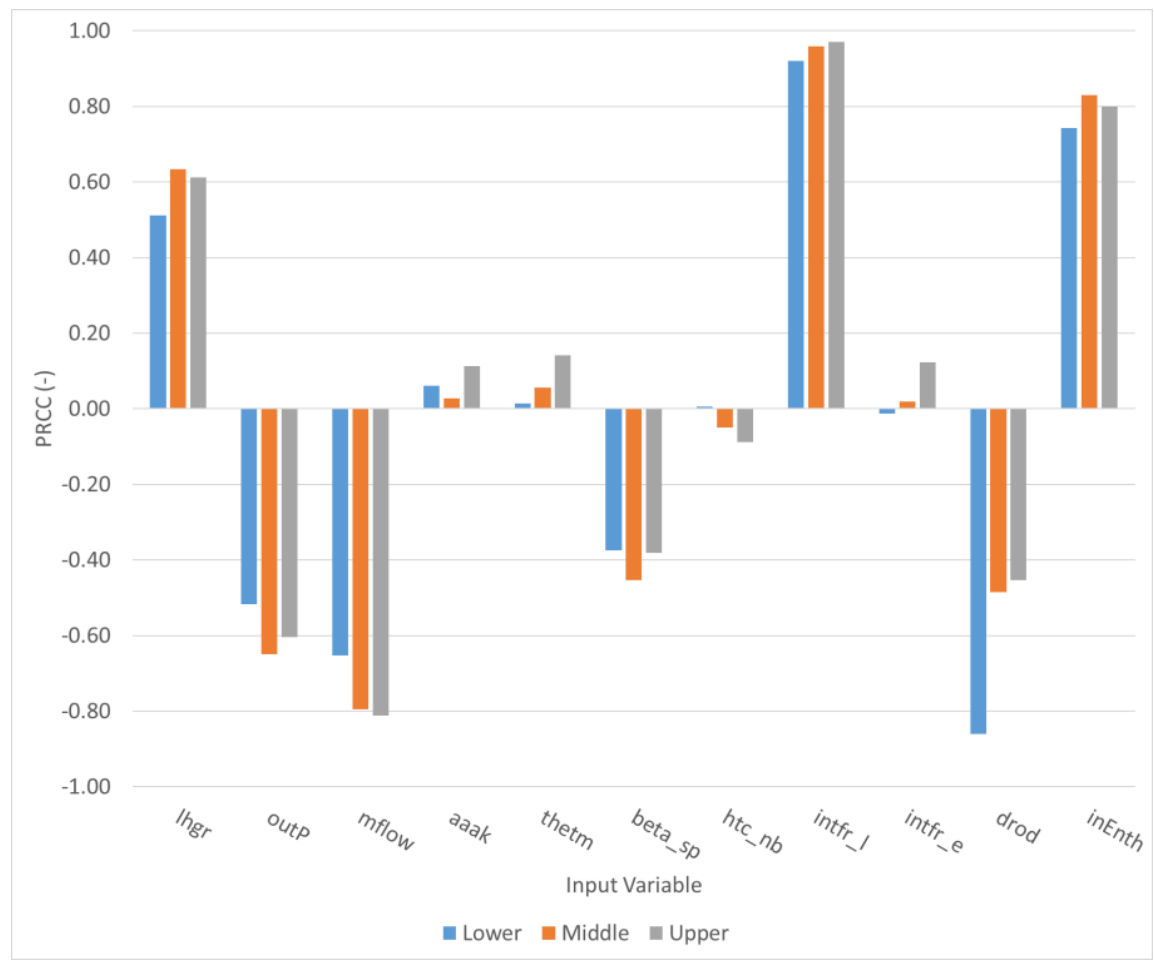

Figure 4.26 Diagram of the PRCCs for Test Run 5.4562. 
Chapter 4. Validation and Verification of Codes and Methodologies for two Application cases

Table 4.20 Sensitivity Analysis of Test Run 5.6321.

\begin{tabular}{|l|l|r|r|r|}
\cline { 3 - 5 } \multicolumn{2}{c}{} & \multicolumn{3}{c|}{ CTF-UPVIS Void Fraction } \\
\hline Input Variable & Label & \multicolumn{1}{c}{ Lower } & Middle & Upper \\
\hline LHGR & lhgr & 0.51 & 0.38 & 0.36 \\
\hline Outlet Pressure & outP & -0.37 & -0.31 & -0.27 \\
\hline Inlet Mass Flow & mflow & -0.67 & -0.57 & -0.60 \\
\hline Eq. distribution weighting factor for void drift & aaak & -0.08 & -0.09 & -0.13 \\
\hline 2-phase multiplier of the mixing coefficient & thetm & -0.04 & -0.04 & -0.23 \\
\hline 1-phase mixing coefficient & beta_sp & -0.64 & -0.71 & -0.78 \\
\hline Nucleate boiling heat transfer coefficient & htc_nb & 0.06 & 0.08 & 0.02 \\
\hline Interfacial drag coefficient for liquid & intfr_l & 0.64 & 0.88 & 0.90 \\
\hline Interfacial drag coefficient for entrainment & intfr_e & 0.08 & 0.05 & 0.07 \\
\hline Rod diameter & drod & -0.97 & -0.92 & -0.87 \\
\hline Inlet coolant enthalpy & inEnth & 0.69 & 0.60 & 0.58 \\
\hline
\end{tabular}

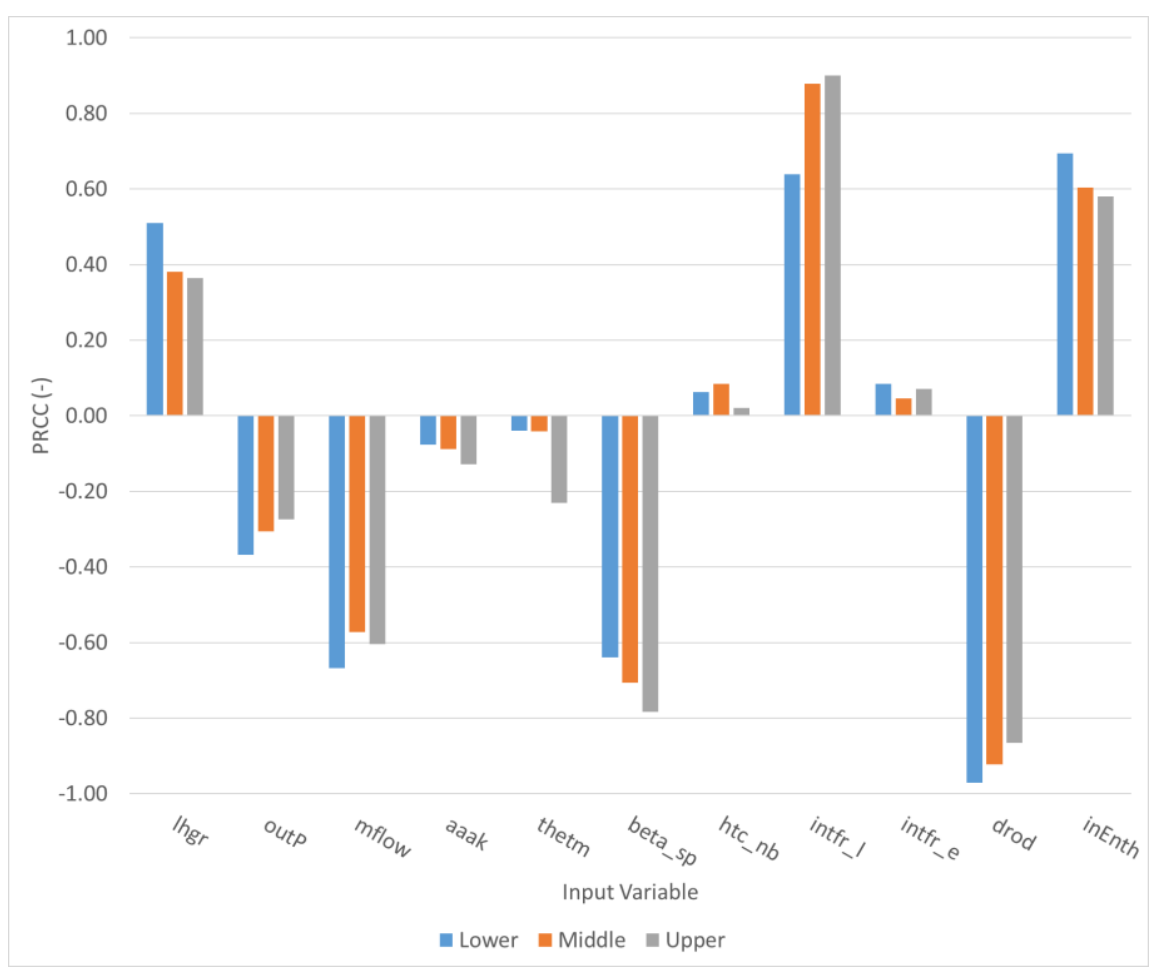

Figure 4.27 Diagram of the PRCCs for Test Run 5.6321. 
Development and validation of a multi-scale and multi-physics methodology for the safety analysis of fast transients in Light Water Reactors

Table 4.21 Sensitivity Analysis of Test Run 5.6322.

\begin{tabular}{|l|l|r|r|r|}
\cline { 3 - 5 } \multicolumn{2}{c}{} & \multicolumn{3}{c|}{ CTF-UPVIS Void Fraction } \\
\hline Input Variable & Label & \multicolumn{1}{c|}{ Lower } & Middle & Upper \\
\hline LHGR & lhgr & 0.37 & 0.32 & 0.26 \\
\hline Outlet Pressure & outP & -0.34 & -0.25 & -0.21 \\
\hline Inlet Mass Flow & mflow & -0.54 & -0.55 & -0.53 \\
\hline Eq. distribution weighting factor for void drift & aaak & 0.06 & -0.02 & -0.13 \\
\hline 2-phase multiplier of the mixing coefficient & thetm & -0.09 & -0.19 & -0.43 \\
\hline 1-phase mixing coefficient & beta_sp & -0.61 & -0.75 & -0.82 \\
\hline Nucleate boiling heat transfer coefficient & htc_nb & 0.01 & -0.02 & -0.05 \\
\hline Interfacial drag coefficient for liquid & intfr_I & 0.90 & 0.91 & 0.90 \\
\hline Interfacial drag coefficient for entrainment & intfr_e & -0.05 & -0.05 & 0.19 \\
\hline Rod diameter & drod & -0.94 & -0.88 & -0.77 \\
\hline Inlet coolant enthalpy & inEnth & 0.61 & 0.58 & 0.55 \\
\hline
\end{tabular}

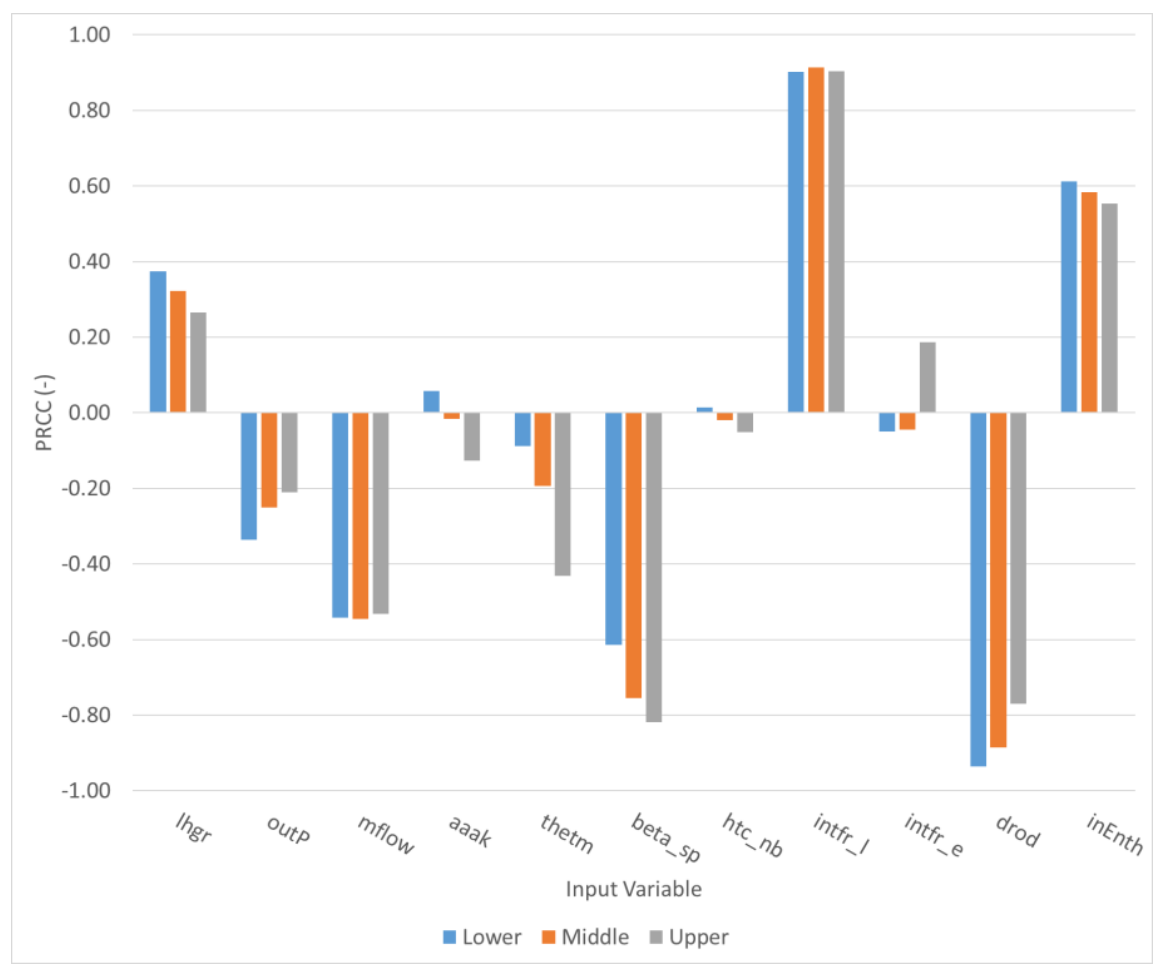

Figure 4.28 Diagram of the PRCCs for Test Run 5.6322. 
Chapter 4. Validation and Verification of Codes and Methodologies for two Application cases

Table 4.22 Sensitivity Analysis of Test Run 5.6552.

\begin{tabular}{|l|l|r|r|r|}
\cline { 3 - 5 } \multicolumn{2}{c}{} & \multicolumn{3}{c|}{ CTF-UPVIS Void Fraction } \\
\hline Input Variable & Label & \multicolumn{1}{c|}{ Lower } & Middle & Upper \\
\hline LHGR & lhgr & 0.52 & 0.64 & 0.60 \\
\hline Outlet Pressure & outP & -0.45 & -0.53 & -0.50 \\
\hline Inlet Mass Flow & mflow & -0.66 & -0.80 & -0.78 \\
\hline Eq. distribution weighting factor for void drift & aaak & 0.07 & -0.05 & 0.01 \\
\hline 2-phase multiplier of the mixing coefficient & thetm & 0.01 & 0.07 & 0.08 \\
\hline 1-phase mixing coefficient & beta_sp & -0.45 & -0.38 & -0.46 \\
\hline Nucleate boiling heat transfer coefficient & htc_nb & -0.01 & -0.09 & -0.12 \\
\hline Interfacial drag coefficient for liquid & intfr_l & 0.96 & 0.96 & 0.99 \\
\hline Interfacial drag coefficient for entrainment & intfr_e & 0.02 & 0.04 & 0.22 \\
\hline Rod diameter & drod & -0.75 & -0.24 & -0.32 \\
\hline Inlet coolant enthalpy & inEnth & 0.67 & 0.73 & 0.69 \\
\hline
\end{tabular}

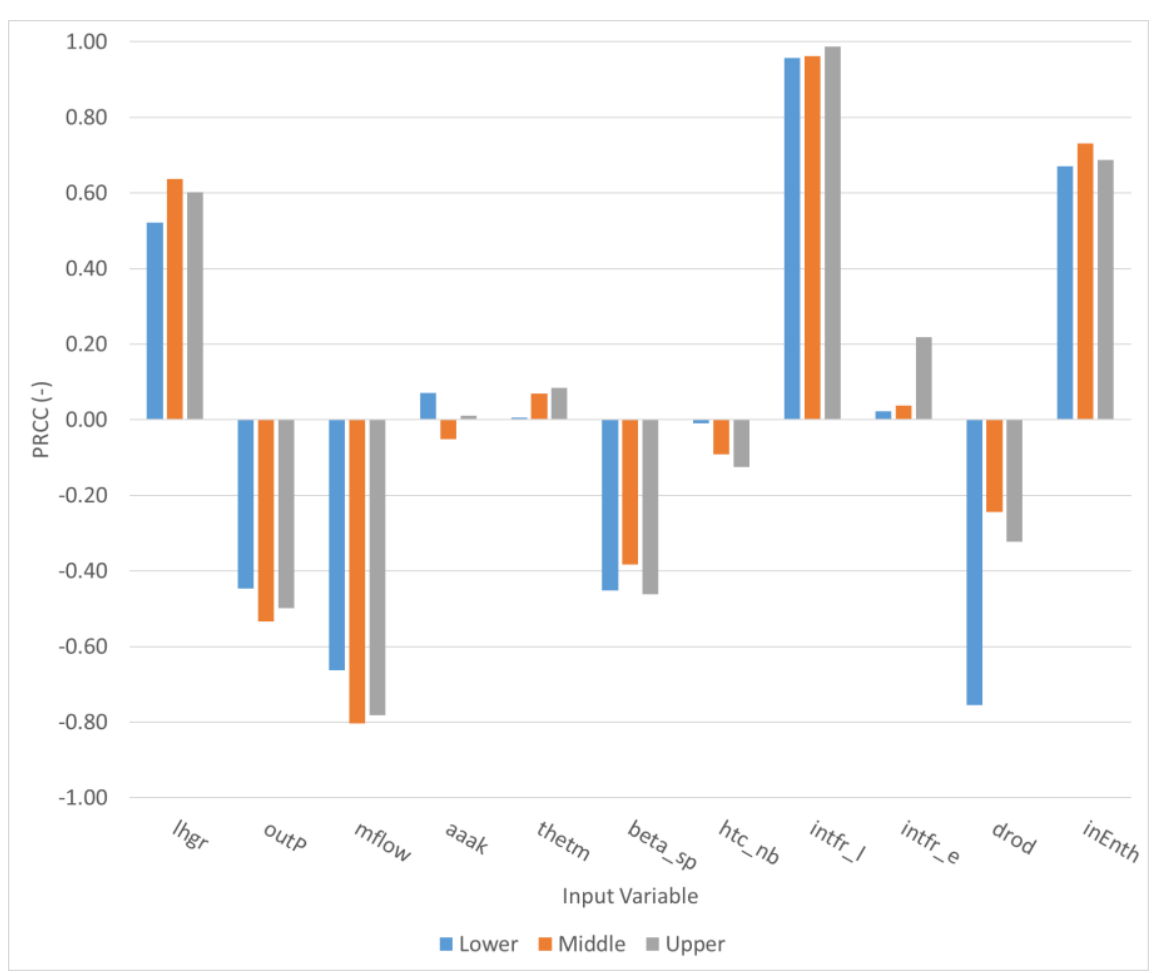

Figure 4.29 Diagram of the PRCCs for Test Run 5.6552. 
Furthermore, internal parameters of the different flow and heat transfer correlations have showed little influence in the uncertainty of void fraction, except for the single phase mixing coefficient and the interfacial drag coefficient of the liquid phase. The former, shows a negative PRCC and the latter a positive one. The single phase mixing coefficient is defined in CTF-UPVIS as the ratio between transverse and axial mass flux.

Consequently, a higher coefficient will increase the part of mass flux in the axial direction in relation with the transversal direction. Therefore, higher values, as for the Inlet Mass Flow contribute to less void fraction. Lastly, the interfacial drag coefficient for the liquid field appears to have more effect on void uncertainty for measurement point locations with higher void fraction. The interfacial drag coefficient relates the transfer of momentum between the liquid field and the vapor field. A higher interfacial drag coefficient allows more transfer of momentum and therefore more void generation. The measurement points detecting more void fraction show higher PRCC values for this coefficient, since there is more vapor field in contact with the liquid field. The SA on the coefficients used in the different correlations modeled in CTF-UPVIS for heat transfer and flow regimes gives an idea of which correlations are more sensible and hence which parameters need special interests for further modeling.

\section{Transient results.}

The following figures show the evolution of the statistical parameters of the sample for the different measurement points, according to the prediction of the void fraction. The statistical parameters are the mean value of the sample and the corresponding confidence interval defined according to the $95 / 95$ criterion.

The Uncertainty Quantification (UQ) has defined the mean value and standard deviation for the predicted void fraction in each time-step. This quantification has been done for the 146-case-sample. Figure 4.30 to Figure 4.32 depict the $95 \%$ Confidence Interval for each time step, calculated from the obtained statistical values from the UQ.

The analysis of the 146 cases of the sample showed that more than $95 \%$ of the sample is included in the $95 \%$ Confidence Interval, meeting the $95 / 95$ criterion stablished by the formula of Wilks.

In regard of the deterministic results, Figure 4.30 to Figure 4.32 shows that the void fraction in every measure point follows a trend equivalent to the evolution of the inlet temperature of the coolant (see Figure 4.13, left). An increase in the temperature of the coolant at the inlet increases the onset of nucleate boiling since the heat transfer needed to achieve saturation temperature will be less. On the other hand, as the experiment shows, when the coolant temperature at the inlet decreases, the void fraction will decrease. 
Chapter 4. Validation and Verification of Codes and Methodologies for two Application cases

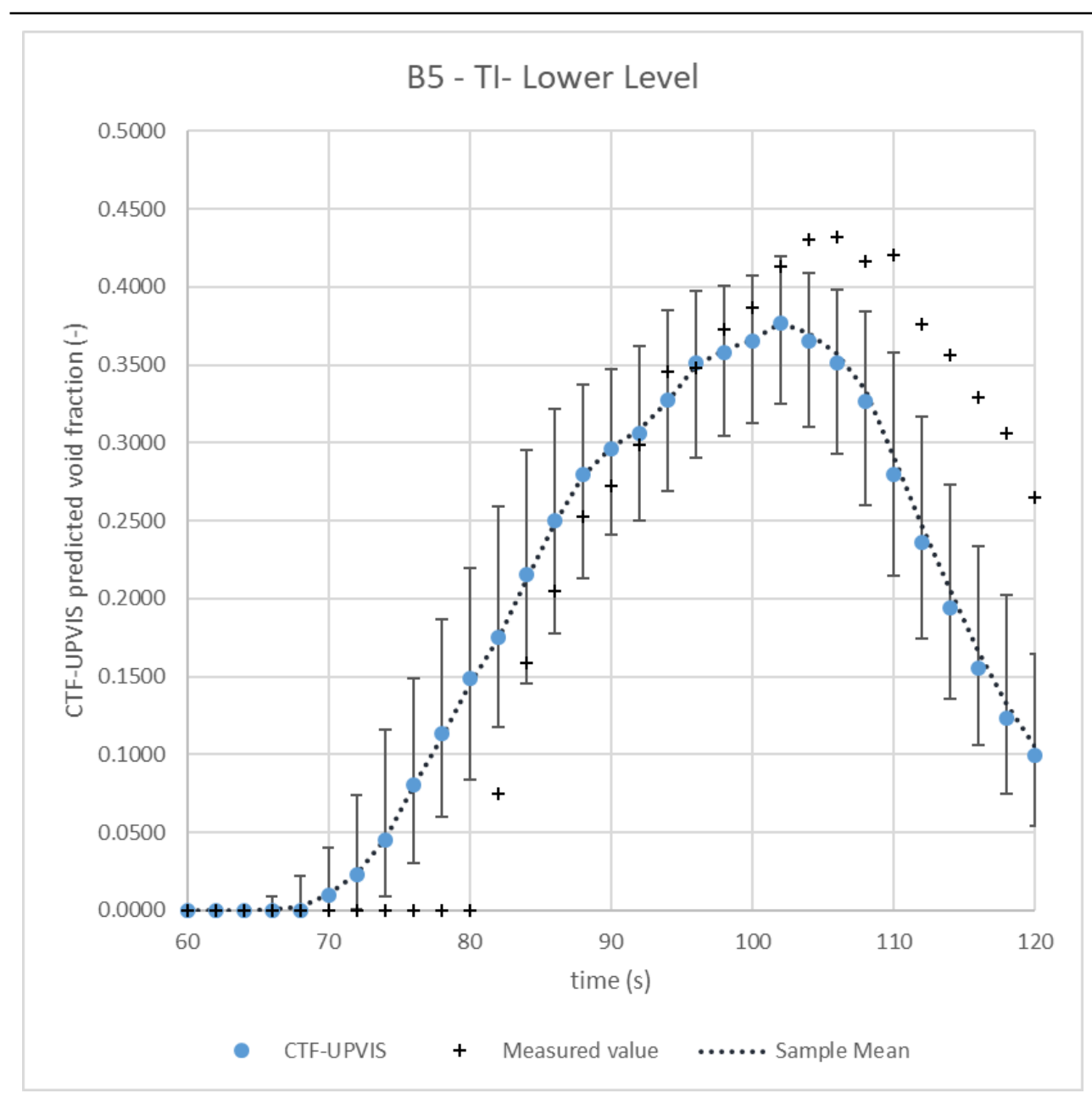

Figure 4.30 Evolution of the void fraction and statistical parameters for the lower measurement point of the PSBT case.

Simulated data shows an overprediction of the void fraction at the beginning of the transient, approximately before the void starts to be detected in the test facility. The void predicted by CTF-UPVIS shows good agreement between the onset of void detection in the test facility and the peak detected around 100 seconds, where the peak of the coolant inlet temperature is located. After the peak, CTF-UPVIS shows an underprediction of the void fraction for every measurement point.

The data provided from the test facility shows no void fraction until the sudden increase when the onset of nucleate boiling is detected by the X-Ray measurement points. CTFUPVIS is capable of predicting a softer evolution of the increase of the void due to its model of small bubble flow regime. CTF-UPVIS shows positive values of void fraction 
Development and validation of a multi-scale and multi-physics methodology for the safety analysis of fast transients in Light Water Reactors

while the test facility is unable to detect void and therefore records a 0 value. The modelling of this flow regime allows a prediction of certain void fraction before achieving the churn flow regime model.

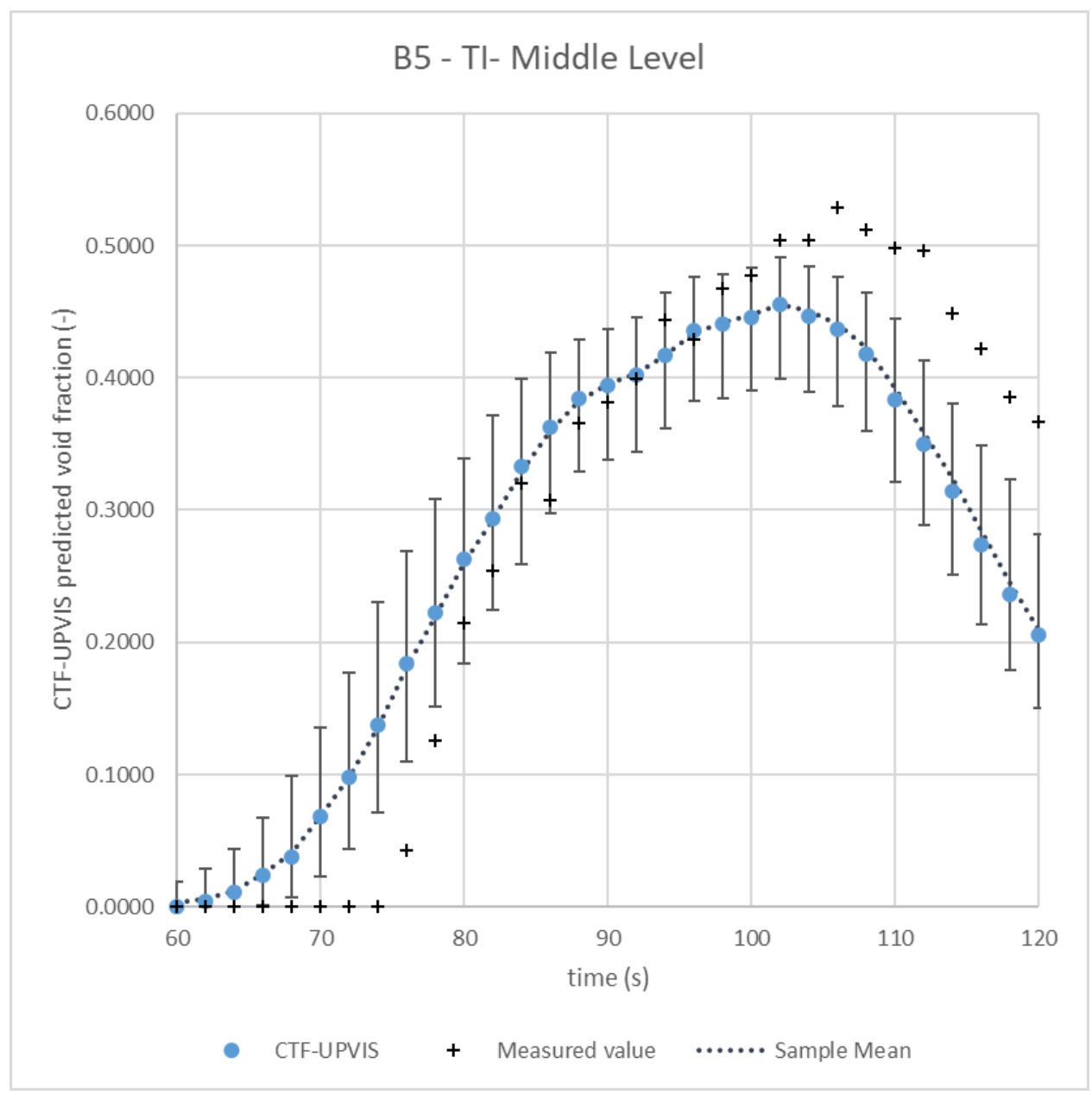

Figure 4.31 Evolution of the void fraction and statistical parameters for middle measurement point of the PSBT case.

The onset of the void fraction takes place earlier in the transient for higher measure points, where the nucleate boiling is achieved earlier do to the approximation to saturated temperature. After the onset of the void fraction, CTF-UPVIS shows good agreement for the prediction of the values. Moreover, the results are more coherent for the higher measurement point, since there is more void generated and detected in comparison with the other measurements. 


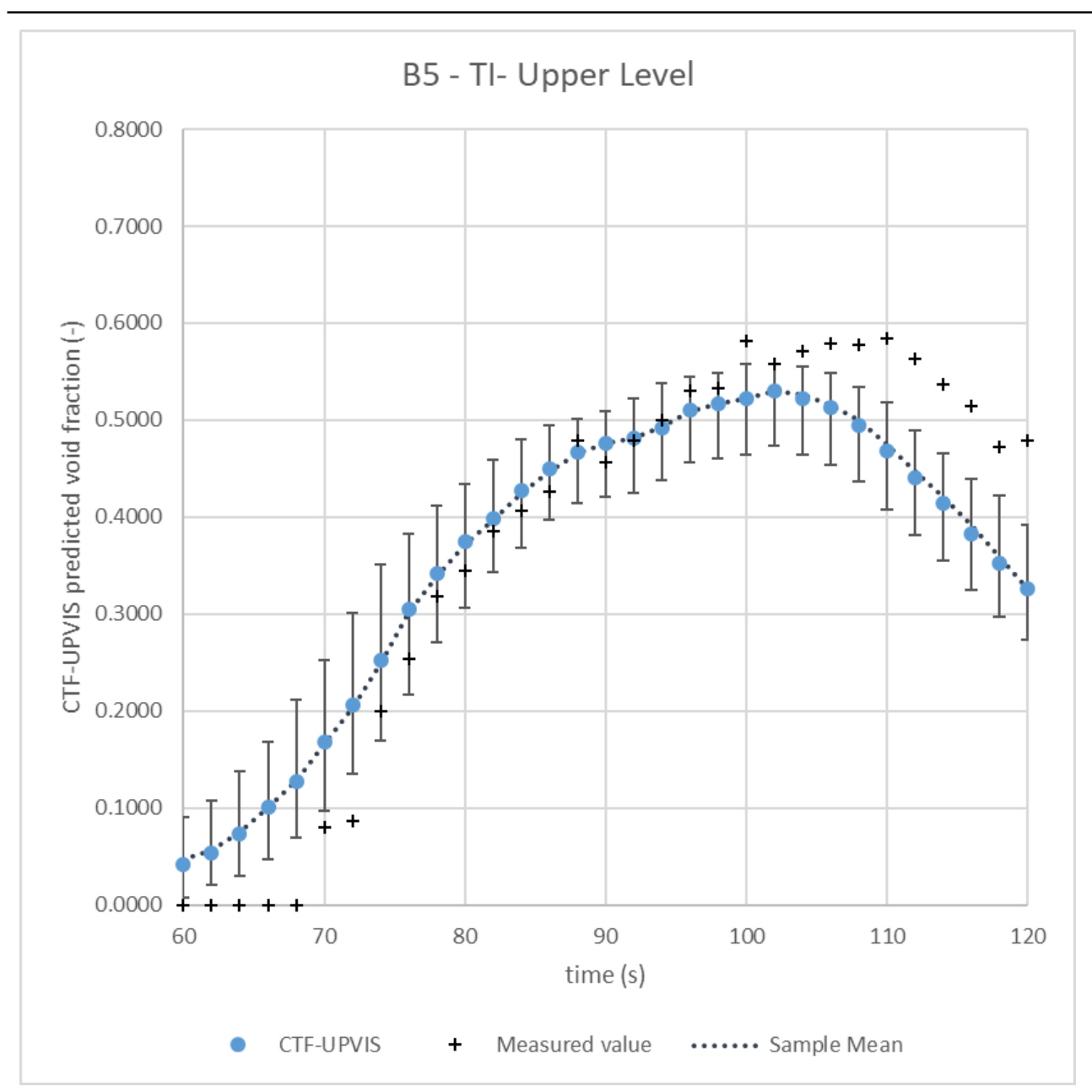

Figure 4.32 Evolution of the void fraction and statistical parameters for the upper measurement point of the PSBT case.

After the peak in the void fraction, corresponding to the peak of coolant inlet temperature, CTF-UPVIS shows an underprediction of the void fraction. The response of the prediction of the void fraction in CTF-UPVIS shows similar behavior to the evolution of the inlet enthalpy of the coolant (see Figure 4.13, left) which is a forced boundary condition. Such behavior can be a consequence of a strong correlation between parameters, that will be confirmed by the Sensitivity Analysis of the results. Even though both measured and simulated values present the same behavior, the values measured in the experiment show certain delay in the decrease of the void fraction with a smaller slope. Moreover, according to Figure 14 (left), pressure in the outlet increases during the transient. A pressure increase raises the saturation temperature, and therefore, generating void in 
Development and validation of a multi-scale and multi-physics methodology for the safety analysis of fast transients in Light Water Reactors

the two-phase flow becomes more difficult. The peak of the void fraction for every measure point is achieved in an increasing pressure trend. Therefore, the model in CTFUPVIS for the void generation is having a sensitive response for such variation of parameters.

Comparison of the results with other participants of the Benchmark.

Different participants from international institutions have shared the results of their codes. This information is added to this section to give a wider picture of the state-ofthe-art of the different subchannel codes.

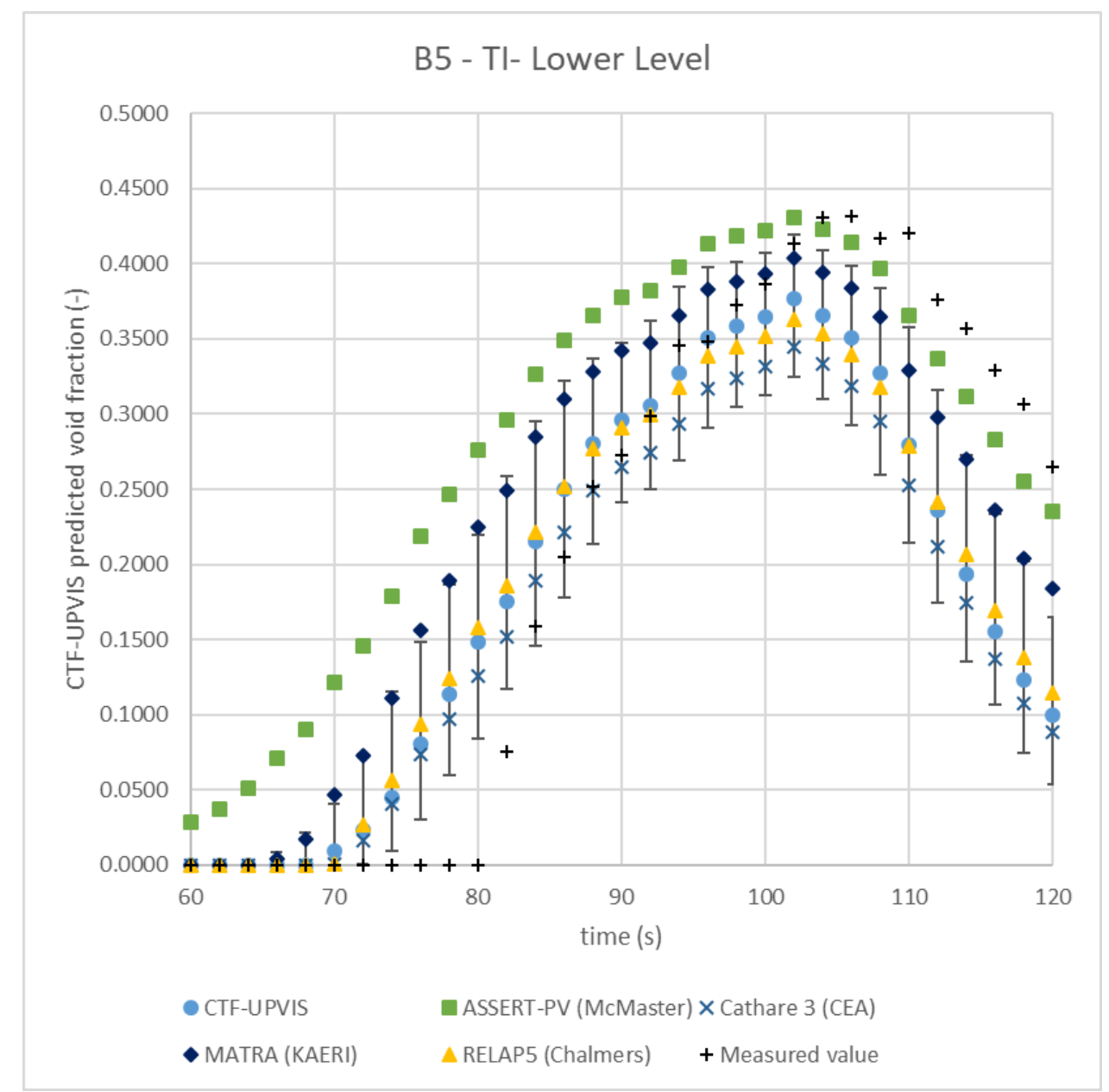

Figure 4.33 Comparison against the results of other PBST participants for the Lower Measurement Point. 
Figure 4.33 to Figure 4.35 show the results of the lower, middle and higher measurement points. For the sake of clarity, the figures show only the comparison with 4 additional participants of the benchmark, all of them using subchannel codes. Further information can be consulted in the benchmark report (OECD/NEA, 2016).

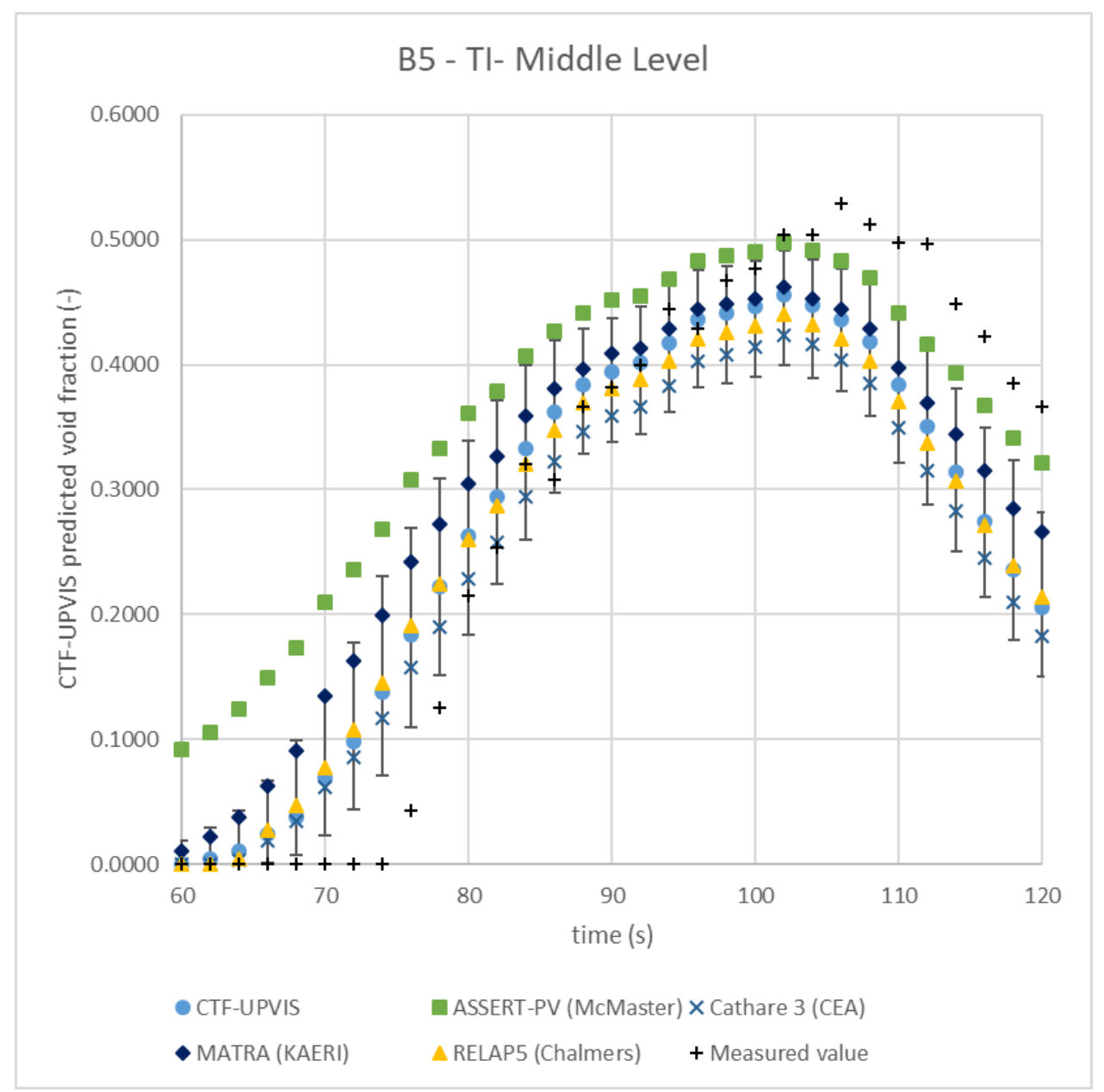

Figure 4.34 Comparison against the results of other PBST participants for the Lower Measurement Point.

In view of the figures, the conclusion is that every participant show good agreement with the trend of the void fraction for the different measurement points. As for CTF, the participants tend to overpredict the void fraction until the void peak at 100 seconds, and underpredict it afterwards. Compared to the rest of the participants, except for FLICA- 
Development and validation of a multi-scale and multi-physics methodology for the safety analysis of fast transients in Light Water Reactors

OVAP, CTF-UPVIS achieves the best agreement for the beginning of the transient until 100 seconds. Conversely, CTF-UPVS shows to be the code underpredicting the void fraction after the peak.

On the other hand, as for CTF-UPVIS, the rest of the participants show better agreement for the measurement points at higher location, where the void is quantitatively higher.

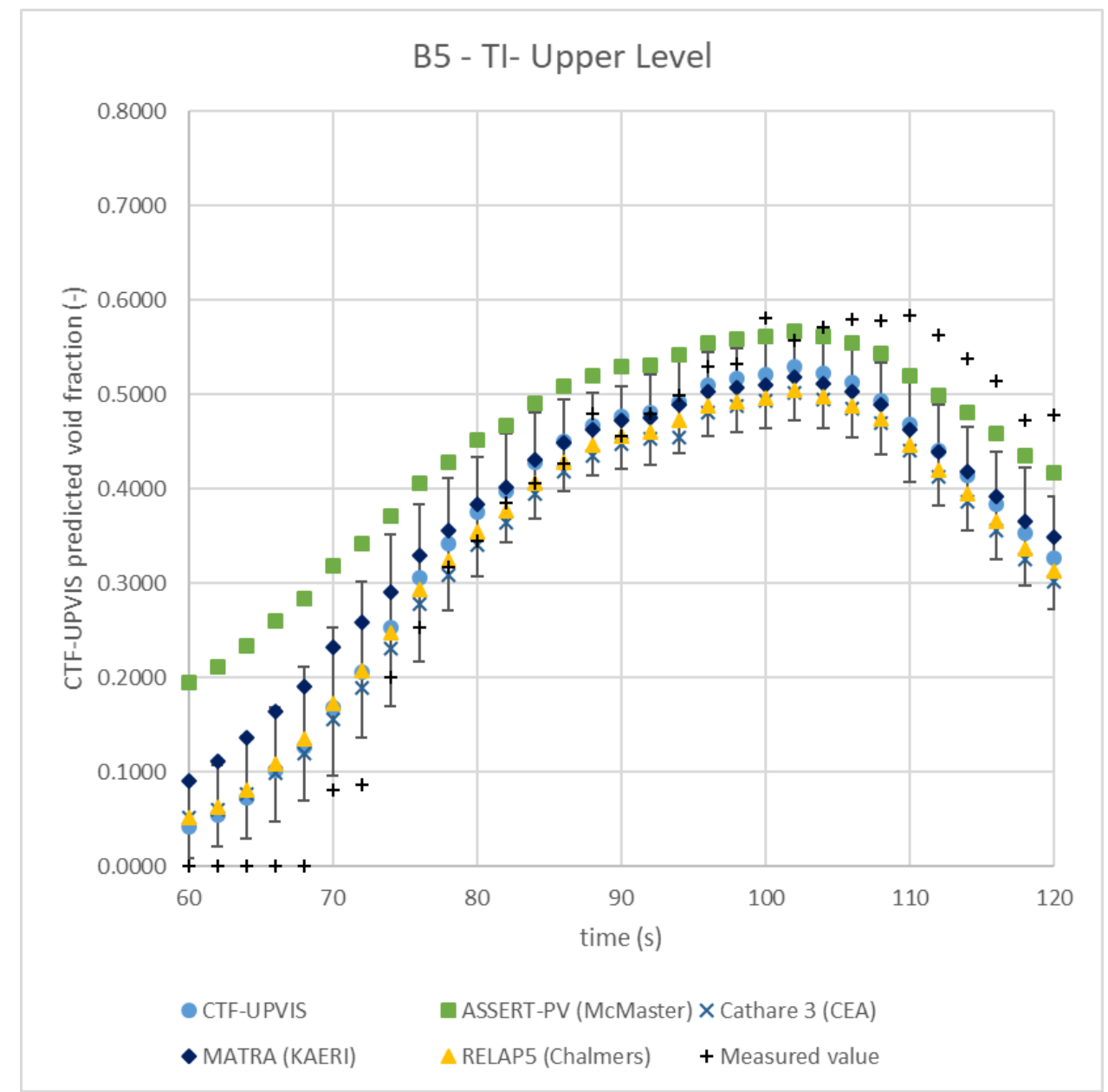

Figure 4.35 Comparison against the results of other PBST participants for the Lower Measurement Point.

The comparison against the results of other participants describes the capacity of the state-of-the-art subchannel codes for predicting the void fraction. As it is known, the different codes can use different correlation for the void fraction and other thermal-hydraulic variables. Moreover, selecting the proper correlation according to the simulation 
Chapter 4. Validation and Verification of Codes and Methodologies for two Application cases

conditions can be a useful method for fitting the results to the experimental data. Further work in regard of the prediction capacity of CTF-UPVIS can consist in extend the sensitivity analysis to other correlation schemes.

Sensitivity Analysis of the transient results.

This subsection analyzes the sensitivity of the prediction of the void fraction regarding the uncertainty of the input parameters. Figure 4.36 to Figure $\mathbf{4 . 3 8}$ show the sets of input variables and their sensitivity along the transient case. As for the steady-state, the sensitivity is quantified using the Partial Rank Correlation Coefficients (PRCC). This measure defines relevant influence in the uncertainty of the output variable for values above 0.2 in absolute value.

Therefore, the inlet enthalpy of the coolant and the outlet pressure are the more relevant simulation boundary conditions, followed by the bundle power and inlet mass flux. On the other hand, void fraction shows less sensitivity to the coefficients of the two-phase flow model implemented in CTF-UPVIS, except for the single-phase mixing coefficient and the interfacial drag coefficient of the liquid field.

The transient analysis of the sensitivity reveals that major changes occur for the inlet enthalpy, which shows a peak in all three measurement points coincident with the evolution of the transient case. The rest of the PRCC show little evolution since they remain constant or with little perturbation.

Moreover, the more sensitive built-in parameters of the two-phase flow model in CTFUPVIS show a peak similar to the peak of inlet enthalpy. This confirms the relation between these parameters and the presence of void. As the void increases, it shows more sensitivity to the interfacial drag coefficient of the liquid field and the single-phase mixing coefficient.

An additional remark is the effect in the PRCC of the initial void fraction. In Figure 4.30, the PRCC of every input variable increases its absolute value at the beginning of the transient. Conversely, for the other two measurement points, the absolute value of the PRCC is reduced. This shows that sensitivity of to the output value increases with its value. At the beginning of the transient, due to the axial location of the ONB, there is no void fraction detected at the lower measurement point. Therefore, the sensitivity experiments a significant increase as the inlet temperature increases and hence, so does the void fraction at that axial node. The contrary effect can be observed for the other measurement points, where there is already void fraction detected. 
Development and validation of a multi-scale and multi-physics methodology for the safety analysis of fast transients in Light Water Reactors

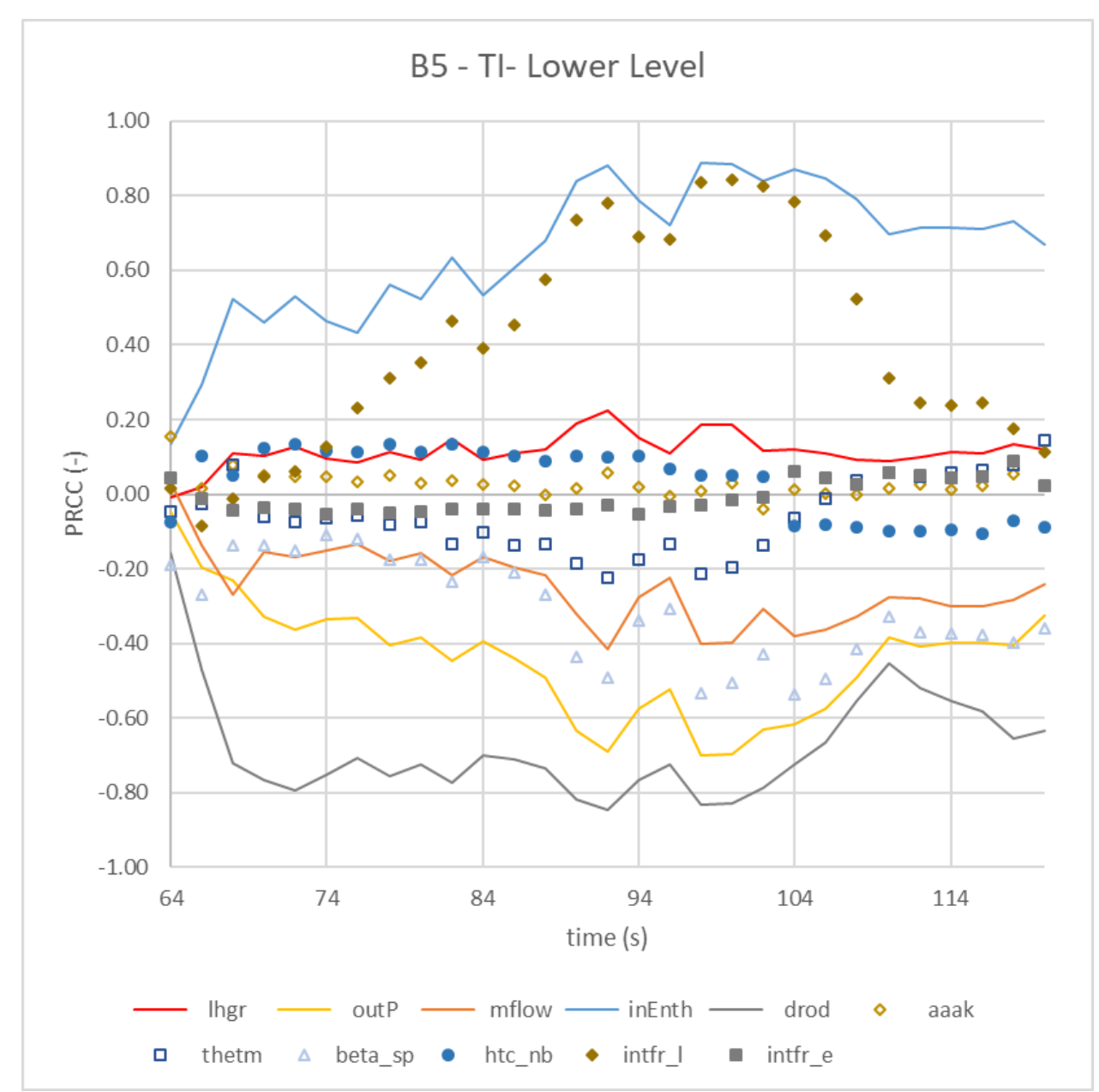

Void prediction is 0.0 for the two first seconds of the transient and therefore PRCC does not show a real number.

Figure 4.36 Sensitivity Analysis of the transient conditions for the Lower Measurement Point. 
Chapter 4. Validation and Verification of Codes and Methodologies for two Application cases

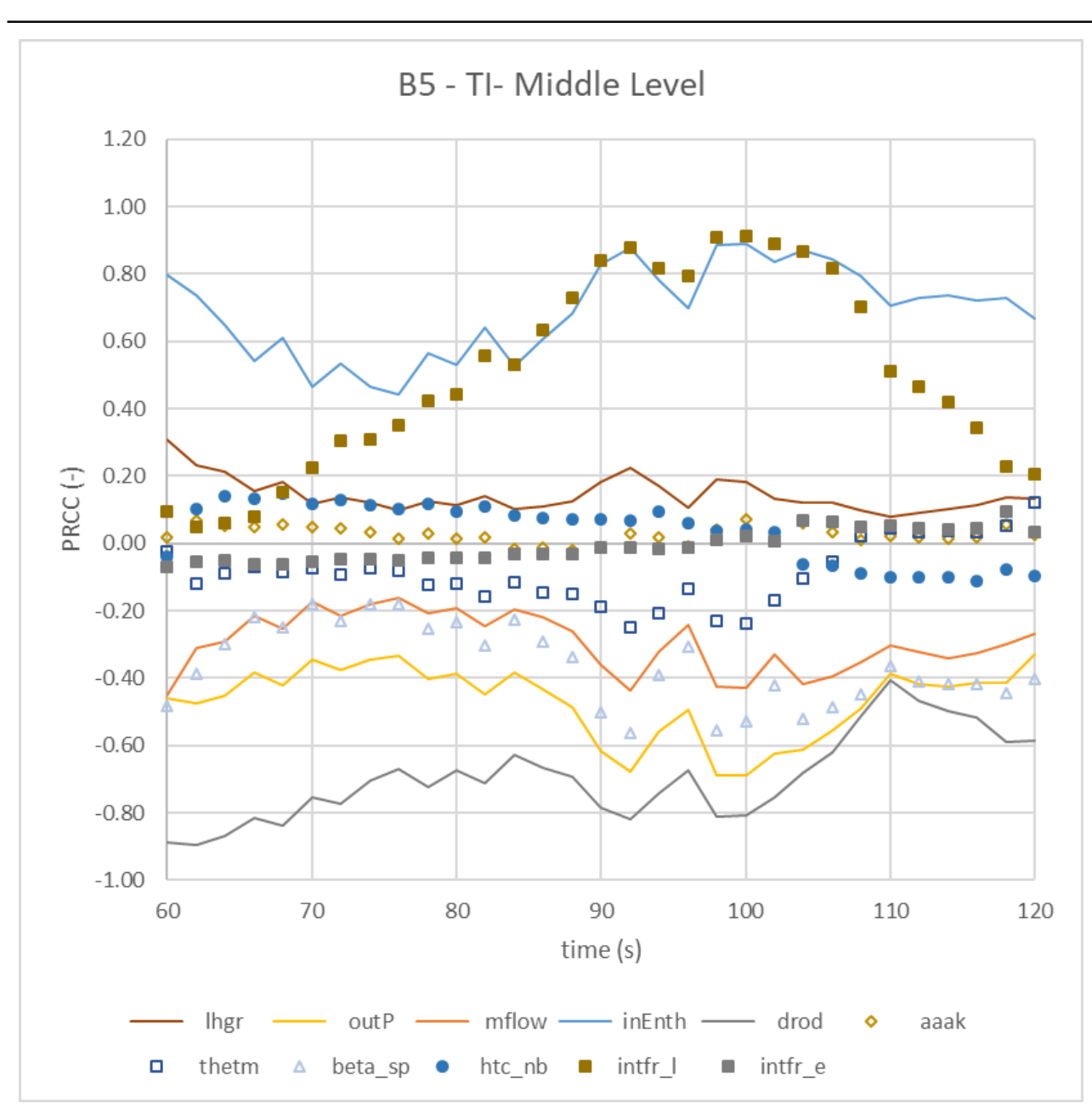

Figure 4.37 Sensitivity Analysis of the transient conditions for the Lower Measurement Point. 
Development and validation of a multi-scale and multi-physics methodology for the safety analysis of fast transients in Light Water Reactors

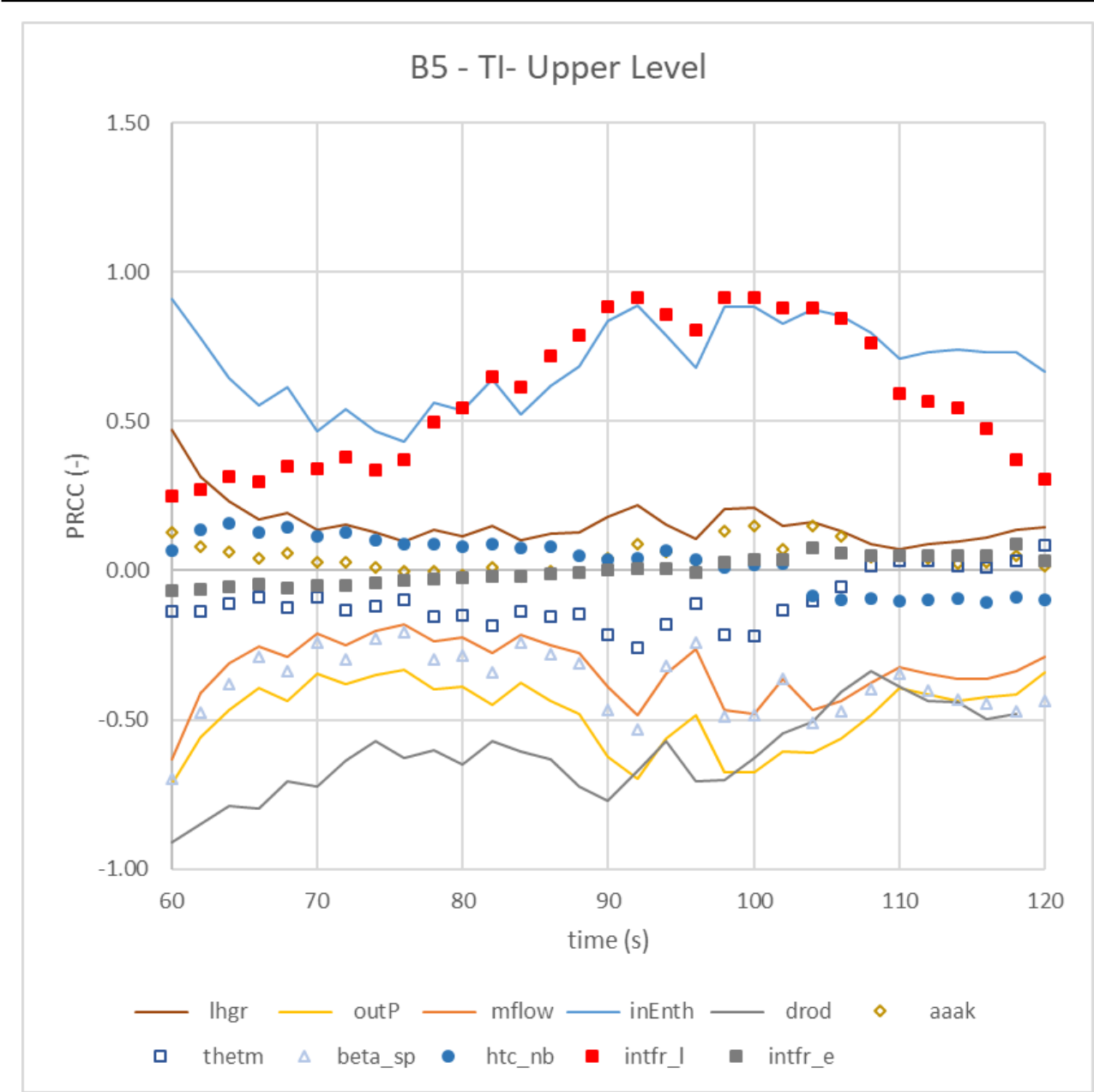

Figure 4.38 Sensitivity Analysis of the transient conditions for the Lower Measurement Point.

\subsubsection{Conclusions.}

The BEPU methodology undertaken by CTF-UPVIS and DAKOTA has been applied successfully to the prediction of the void fraction for the benchmark PSBT of the NEA/OECD for 11 steady-state test cases and the transient case of coolant inlet temperature increase.

The results predicted by CTF-UPVIS showed good agreement with the measured values and additionally, good agreement compared to the prediction of other participants. This confirms that the use of subchannel codes accounting the transvers effects of the flow between rod arrays are of significant importance to model local phenomena, like in this case, the prediction of void fraction. The results show good agreement of CTF-UPVIS 
Chapter 4. Validation and Verification of Codes and Methodologies for two Application cases

for the prediction of the evolution of the void fraction. Moreover, CTF-UPVIS showed good agreement with the measurements of the experiment for the middle part of the transient. The discrepancies with the measurement data are observed in the parts of the transient where the boundary variable of the inlet coolant temperature changes from increasing to decreasing. The prediction of CTF-UPVIS revealed significant sensitivity to the change of this parameter, while the measurement was in comparison less sensitive. This hypothesis has been confirmed by the SA of the results. In addition, the discrepancies at the beginning of the transient are considered to be related to the measurement capacity of small bubbles of void fraction. The experiment shows a sudden increase in void fraction when it starts to being detected, while the small bubble flow regime of CTF-UPVIS predicts a softer increase of void fraction.

The results have been complemented with an Uncertainty and Sensitivity Analysis. The use of DAKOTA toolkit has provided the sampling procedure and the quantification of the CI. Due to this, it can be observed that the uncertainty of the predicted value can include the measured value with a $95 \%$ of probability.

The Sensitivity Analysis showed significant relevancy of the inlet enthalpy in the uncertainty of the prediction of the void fraction. In addition, certain correlation parameters such as the interfacial drag coefficient for the liquid field and the single phase mixing coefficient showed to be sensitive for the prediction of the void fraction. 


\section{Chapter 5}

\section{Application of the proposed methodology to the Safety Analysis of the Turbine Trip Event of KKL in Fuel Cycle 18}

The preliminary results of the application of the methodology are going to be validated against plant data of a real scenario. This scenario consists of a Turbine Trip (TT) event with Bypass System available that happened in KernKraftwerk Leibstadt at the beginning of fuel cycle 18, i.e. September of 2001. The selected event is part of the Design Basis of a NPP with a GE BWR/6 design, that counts on a steam cycle that feeds a turbine-generator group. Therefore, the Safety Analysis in a NPP requires of the assessment of this event in a regular basis. This means that the Nuclear Regulator will ask for the assessment of this event in different times along the Operational Life of the nuclear facility. For instance, whenever a NPP modification affects any of the systems related to the Turbine, or the systems controlling its operation or safety functions, this event will be re-evaluated to give credit that the modification does not compromise the safety of the plant or that this transient is still managed safely.

Moreover, this transient case is selected among the available plant data due to its multiphysic behavior. The evolution of the core parameters during this transient include a 
Chapter 5. Application of the proposed methodology to the Safety Analysis of the Turbine Trip Event of KKL in Fuel Cycle 18

strong feedback between the NK and the TH. In addition, in regard of further fuel behavior analysis, it allows to extend the application of the proposed methodology to different codes and scales.

The simulation conditions and the plant results have been provided by KKL in the framework of a collaboration project. This plant conditions have been analyzed and used to validate the core behavior.

This chapter describes the application case in the first section. The next section, Section 5.2, details how the methodology has been applied in the different steps and how the generated data has been pre- and post-processed. Section 5.3 presents the results obtained at each step with the corresponding discussion. And the last section summarizes the conclusions of the analysis of the results.

The development of the presented methodology has taken advantage of the plant data available, and the first approaches of the application of the methodology have been used to yield different publication in national and international congresses as well as two publications in journal papers (Patricio Hidalga et al., 2019a), (Patricio Hidalga et al., $2019 \mathrm{~b}$ ). The contents of these journal papers are available on Riunet online service provided by the Universitat Politècnica de València.

\subsection{Case description.}

According to the USNRC on its Standard Review Plan of Chapter 15.2.1 (USNRC, 2007c) to 15.2.5 (USNRC, 2007b), a Turbine Trip With Bypass Available event is an initiating event that can occur with moderate frequency and has diverse causes that can explain its occurrence. A Turbine Trip consists on the fast closure of the Turbine Control Valve (TCV), and in this case, with the consequent opening of the Turbine Bypass Valve (TBV). In this manner, the main heatsink (the condenser) is still available and the power generated in the reactor can be delivered. A more severe accident of this nature would be the TT without Bypass. In that case, the Bypass Valve system fails to open, and therefore the main heatsink is lost. Figure 5.1 shows the layout of the coolant/steam flow of the affected systems. 
Development and validation of a multi-scale and multi-physics methodology for the safety analysis of fast transients in Light Water Reactors

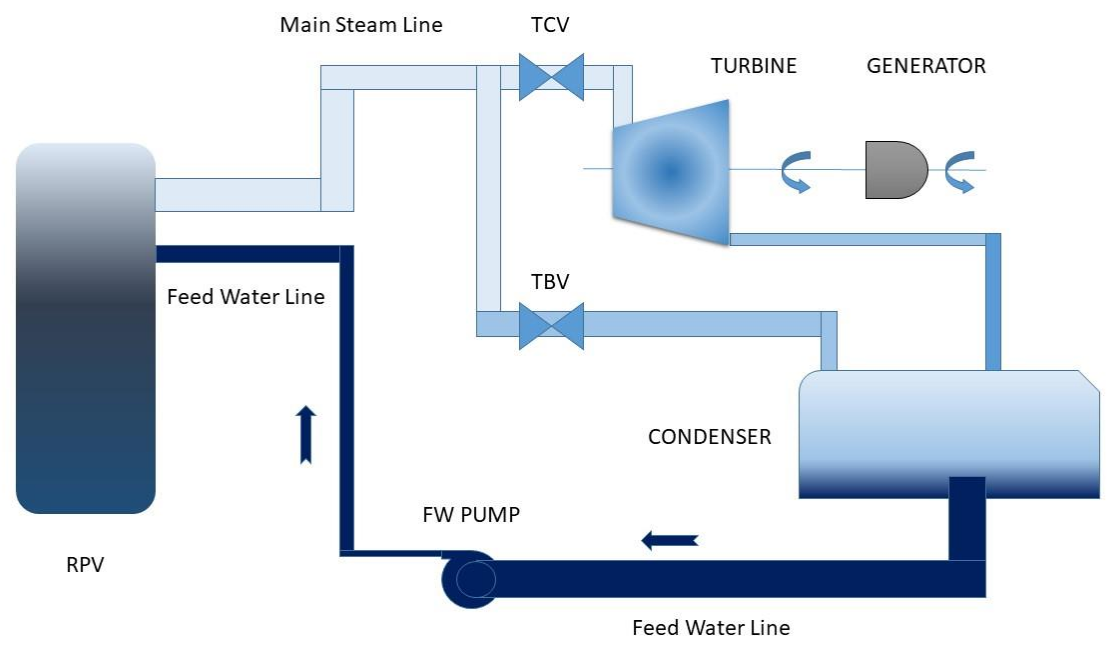

Figure 5.1 Steam flow through the affected systems.

The trip system of the turbine has a protective function and can be triggered by different signals that detect potential initiating events that can affect the turbine-generator group. For instance, the overpressure in the Turbine Building, the loss of vacuum in the condenser or a Loss of Onsite Power can trip the Turbine. The consequence is a fast transient event that causes a pressure peak upwards the turbine group, i.e. the main steam lines and afterwards the Reactor Pressure Vessel. The pressure peak is a consequence of the rejection of the steam load happening in the milliseconds when the TCV closes while the TBV starts to open. Figure 5.2 describes the magnitude of the velocity of the first time steps of this transient. In Figure 5.2 and Figure 5.3 it is possible to view the movement of the TCV and TBV. The four TCV are labeled as valves A, B, C and D and correspond to each of the steam lines. In the same way, TBVs are labeled as N, N/M, S and S/M. The figures are obtained from the previous validation work in KKL of the TRACE model (Papadopoulos \& Sekrhi, 2017). 

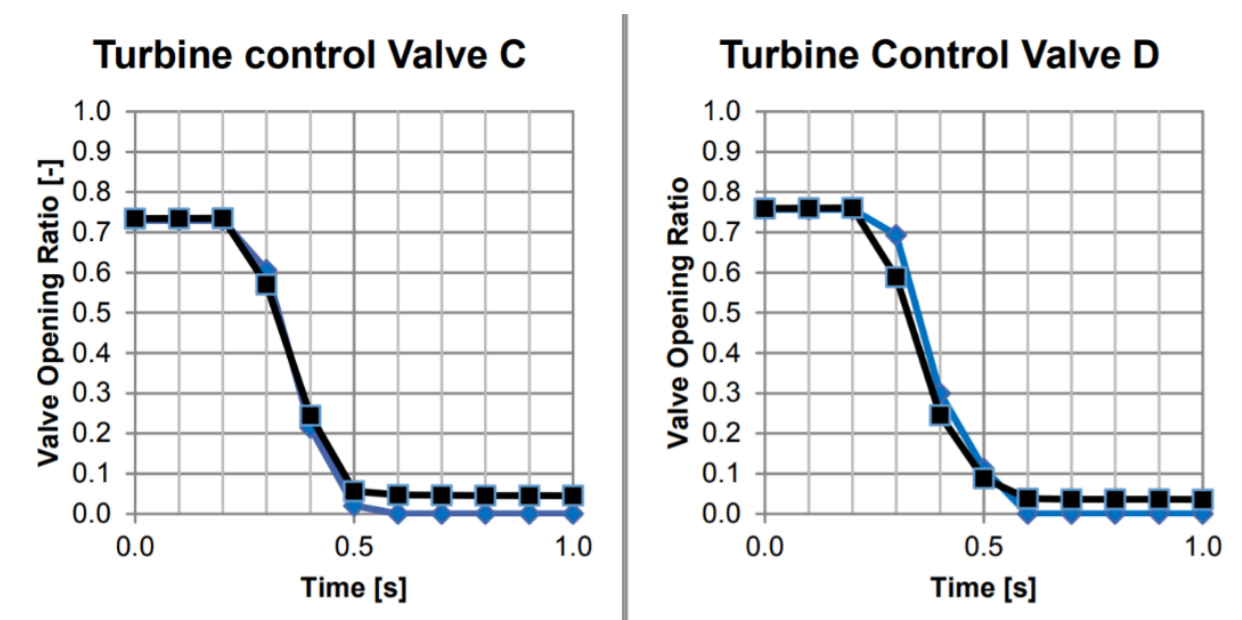

\section{Turbine control Valve $\mathrm{C}$}

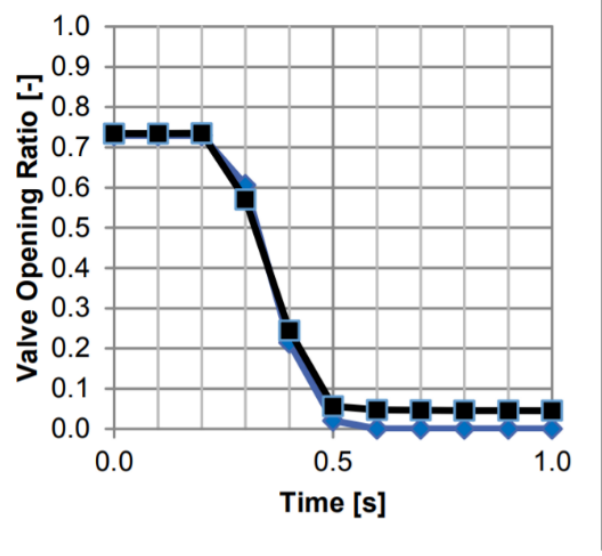

\section{Turbine Control Valve D}

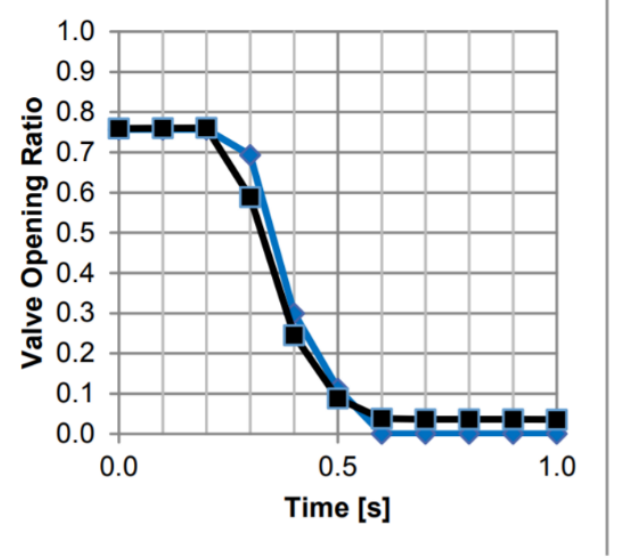

Figure 5.2 TCVs movement during the transient event.

The direct consequence of the pressure peak is an excursion of power in the reactor core. This power excursion is caused by the negative reactivity coefficient of the void fraction. A high void fraction in the reactor diminishes the moderator capacity of the coolant and hence, the fission rate. 
Development and validation of a multi-scale and multi-physics methodology for the safety analysis of fast transients in Light Water Reactors

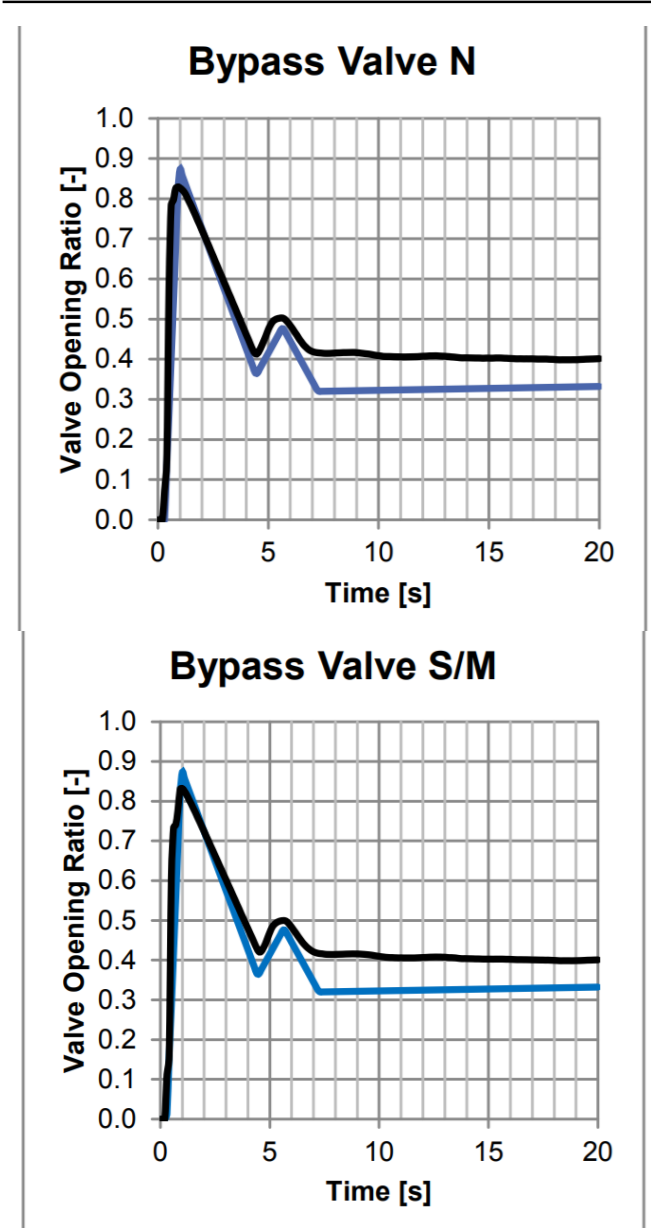

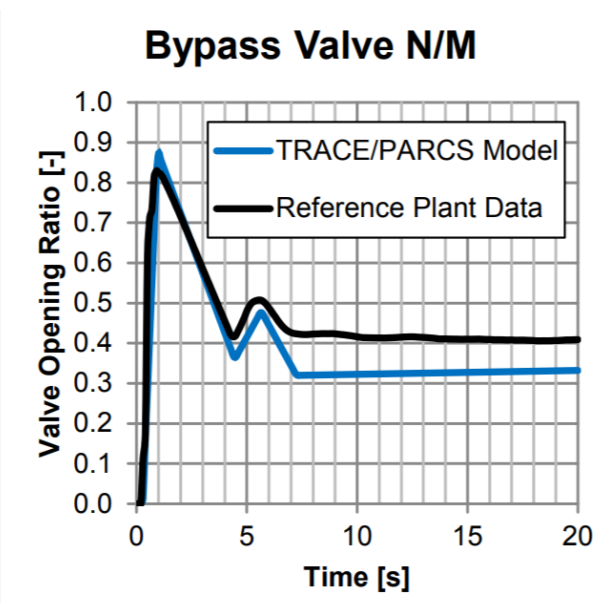

Bypass Valve S

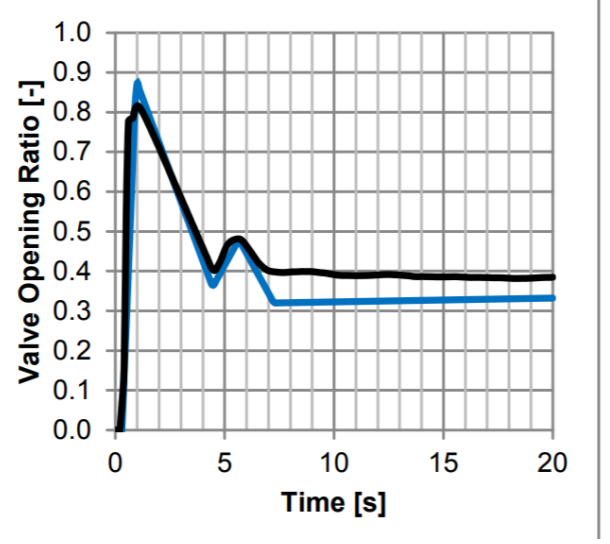

Figure 5.3 TBVs movement during the transient event.

Conversely, a pressure peak collapses the void in the reactor and moderator capacity suddenly increases. According to this event sequence, two main barriers can be affected in this transient. On the one hand, the Reactor Coolant Pressure Boundary (RPCB) and the Reactor Pressure Vessel (RPV) endure a pressure peak that challenges their mechanical integrity. On the other hand, the power peak can yield fuel damage if this power is not properly removed by the coolant. Nevertheless, the velocity of the transient can affect the flow and heat transfer regimes of the coolant surrounding the fuel rod, and the heat removal capacity can be significantly affected.

For the former barrier, it is necessary to assess the integrity of the RPV and RCPB. The USNRC refers to the American Society of Mechanical Engineers (ASME) as a renowned Institution for setting the Acceptance Criteria in pressurized vessels and components. In 
Chapter 5. Application of the proposed methodology to the Safety Analysis of the Turbine Trip Event of KKL in Fuel Cycle 18

this case, the standards define the hydraulic pressurized components must be manufactured to withstand $110 \%$ of their maximum nominal pressure.

For the later barrier, USNRC refers to the MCRP for evaluating a sufficient capacity for removing the heat generated in the cladding. This Acceptance Criteria must be accomplished with a statistical assessment of $95 \%$ of probability of a $95 \%$ Confidence Interval. The MCRP is defined according to the Design of the corresponding NPP, in this case KKL. Due to Design Property class, this value is not allowed to be published and therefore, a fictitious estimated value is provided as reference. A mathematical limit for this Acceptance Criterion would be a value of 1 of the MCPR (see section 2.5). Values below 1 means that the generated power is greater than the critical power that causes transition to film boiling and hence a sudden deterioration of the heat removal capacity.

The following protection measure in a Turbine Trip Event With Bypass Available (here on TT) is the SCRAM in order to avoid the power excursion.

The probability of this event is so that a turbine trip can be expected more than once in the operational life of the NPP, according to different Regulators. The frequency of this event assigns in Switzerland the Category of Operational Disturbance (Betriebsstörung, in german) (ENSI, 2018) and this category assigns the acceptance criteria according to the Swiss law (ENSI, 2019). It is common in the Nuclear Regulation Guidelines of the US that certain limits for the Acceptance Criteria are directly defined in the corresponding guideline. However, in Switzerland, it is more common to set which barrier is compromised and assure the integrity of the barrier according to the design basis of this barrier. The analysis of the sequence of events in this transient, together with the postulated protection sequence defines the barrier or barriers that can be compromised during the evolution of the transient case.

Regarding the evolution of the fluid, the focus must be located in the pressure and flow wave behavior, especially at the beginning of the transient. The fast closure of the control vales causes a density wave that travels along the steam line causing a pressure peak in the dome of the vessel. This fact affects directly to the void fraction distribution of the core and hence to the neutron flux distribution. The consequences result in an increase of the reactor coolant temperature, a decrease in the coolant density and an increase in the reactor coolant pressure. This is mitigated by the actuation of Turbine Bypass Valves (TBV) and Safety Relief Valves (SRV) that reduces the pressure peak in the vessel. Moreover, the power peak caused by the pressure peak is controlled by means of Control Rods Maneuver, whether by a Selected Rod Insertion (SRI) or a SCRAM. Table 5.1 describes the sequence of events of the TT, which has been extracted from the corresponding internal report of KKL. 
Development and validation of a multi-scale and multi-physics methodology for the safety analysis of fast transients in Light Water Reactors

Table 5.1 Turbine Trip Fuel Cycle 18 sequence of events.

\begin{tabular}{|c|c|c|c|}
\hline Time (ms) & Event & Time (ms) & Event \\
\hline 0 & Core flow at $3370.85 \mathrm{~kg} / \mathrm{s}$ & 490 & TBVs at $\sim 50 \%$ \\
\hline 0 & Dome pressure at 71.9 bar & 780 & $\begin{array}{c}\text { Peak Vessel Dome Pres- } \\
\text { sure } 7.31 \mathrm{MPa}\end{array}$ \\
\hline 0 & $\mathrm{TT}$ & 1020 & $\begin{array}{l}\text { TBVs reached maximum } \\
\text { opening } \sim 82 \%\end{array}$ \\
\hline 218 & TSVs begin to close & 1410 & SRI Rod Full-In \\
\hline 260 & TBVs begin to open & 2030 & $\begin{array}{l}\text { Peak Steam Flow } 2170.7 \\
\mathrm{~kg} / \mathrm{s}\end{array}$ \\
\hline 300 & TSVs at $\sim 9.125 \%$ & 4880 & $\begin{array}{l}\text { Flow Control Valves at } \\
18 \%\end{array}$ \\
\hline 300 & SRI Channels initiation & 9100 & $\begin{array}{l}\text { Core flow after transient } \\
\text { at } \sim 1892 \mathrm{~kg} / \mathrm{s}\end{array}$ \\
\hline 305 & $\begin{array}{l}\text { Flow Control Valves closed at } \\
\qquad 66 \%\end{array}$ & 9980 & Power Peak at $\sim 58 \%$ \\
\hline 440 & TBVs at $\sim 25 \%$ & 29980 & $\begin{array}{l}\text { Vessel Dome Pressure } \\
6.80 \mathrm{MPa}\end{array}$ \\
\hline
\end{tabular}

The TT event has been analyzed according to the corresponding regulatory documentation. For this case, the USNRC standards have been reviewed in order to elucidate the corresponding figure of merit to be analyzed in this simulation scheme. The figure of merit will be the safety variable that will be evaluated as an output of the further simulations performed in the different steps of the application of this methodology. For that purpose, it is necessary to review the General Design Criteria (GDC) gathered in the USNRC document 10 CFR Appendix A to Part 50 (USNRC, 2017a). Among the criteria presented in this document, only the ones within the scope of the application of a simulation methodology are of interest, being:

- GDC 10, as to reactor coolant system design with appropriate margin so Specified Acceptable Fuel Design Limits (SAFDLs) are not exceeded during normal operation, including Anticipated Operational Occurrences (AOOs). 
Chapter 5. Application of the proposed methodology to the Safety Analysis of the Turbine Trip Event of KKL in Fuel Cycle 18

- GDC 15, as to design of the reactor coolant system and its auxiliaries with appropriate margin so the pressure boundary is not breached during normal operations, including AOOs.

These criteria are afterwards complemented with the American National Standards as guidance. Therefore, the criteria exposed above yield in the following requirements:

- Pressure in the reactor coolant and main steam system should be maintained below 110 percent of the design values.

- Fuel cladding integrity must be maintained by the minimum Departure from Nucleate Boiling Ratio (DNBR) remaining above the 95/95 DNBR limit for PWRs and the Critical Power Ratio (CPR) remaining above the Minimum CPR safety limit for BWRs based on acceptable correlations and satisfaction of any other SAFDL applicable to the particular reactor design.

Therefore, the figure of merit will be the Minimum CPR observed during the simulation of the transient event, as well as the pressure observed in the dome. The reader must notice that the Minimum CPR is a very local phenomenon hence, having a coarse definition of the problem in the simulation model would head to assume a conservative approach. It is here then, where the multi-scale feature of the presented methodology will show the added value to the safety analysis.

\subsection{Road map of the application of the methodology.}

As commented in the previous subsection, the figures of merit are the pressure in the dome and the Minimum CPR. The methodology will be applied starting from a system model of coupled TH and NK where the reactor core is simplified, and will end in a fuel pin analysis that will predict the Minimum CPR of the critical node. Each step will be fed with the boundary conditions predicted in the previous step, enhancing the scale level and accounting the necessary physics for analyzing the target phenomenon. In regard of the pressure of the dome, the first step of the simulation methodology will provide such value, since it is the only step modelling the vessel.

The proposed methodology uses different simulation codes to accomplish the targets of each step. Table 5.2 shows the information of each code and how they will be referred further on. 
Development and validation of a multi-scale and multi-physics methodology for the safety analysis of fast transients in Light Water Reactors

Table 5.2 Information of the simulation codes used in the application of the proposed methodology.

\begin{tabular}{|c|c|c|c|}
\hline $\begin{array}{c}\text { Code abbre- } \\
\text { viation }\end{array}$ & Code version & Developer & Property \\
\hline CASMO & casmo4-2.05.14 & Studsvik/KKL & KKL \\
\hline SIMULATE & simulate3 & Studsvik/KKL & KKL \\
\hline SIMTAB & simtab-v37 & Senubio (ISIRYM/UPV) & UPV \\
\hline PARCS & parcs_m16_UPVIS_ & U. MICHIGAN & USNRC \\
& v1801_ifr & & \\
\hline TRACE & Trace-v50p3 & ISL and NRC & USNRC \\
\hline CTF-UPVIS & CTF_UPVIS_v1701 & Senubio (ISIRYM/UPV)/CTF & UPV/CTF \\
& Users \\
& Uro_x64_r & Uroup \\
\hline FRAPCON & Frapcon-4.0 & PNL & PNL \\
\hline FRAPTRAN & Fraptran-2.0 & PNL & PNL \\
\hline DAKOTA & Dakota-6.4 & Sandia National Laboratories & $\begin{array}{c}\text { Sandia } \\
\text { National }\end{array}$ \\
& & & Laborato- \\
ries
\end{tabular}

Consequently, the application of the different steps will be as follow:

1) Generation of the 3D cross-section libraries that will provide the neutron composition of the core defined for the burnup step when the transient case took place. For this step, the interface SIMTAB will be used fed from the CASMO/SIMULATE-3 data. This step will generate the NEMTAB libraries that will be used by the 3D NK code PARCS.

2) Simulation of the system model TRACE/PARCS-NEMTAB. This step will simulate the overall behavior of the reactor core and the most relevant parts of the primary loop. The model accounts 1D components such as pipes, vales and pumps as well as the 3D Cartesian vessel containing the jet pumps, team separators and the reactor core. The reactor core lumps the fuel channels in three radial averaged channels. This feature allows an efficient computational cost simulation that allows evaluating the behavior of the core. The core is coupled 
with a channel-by-channel core model that will calculate the evolution of the NK with the feedback of the TH.

3) The results of this step are used to implement the boundary conditions of the pressure and core mass flow in a more detailed core model. This core model is a channel-by-channel core modelled with CTF-UPVIS and coupled to the PARCS model suing the NEMTAB files. This step simulates a more detailed scale that allows tracking the critical fuel channel by applying the Minimum CPR criterion.

4) The next steps will use the boundary conditions of the critical fuel channel in the corresponding subchannel model that will simulate the TH stand alone. This step is a high detail thermal-hydraulic model accounting the water rods geometry as well as the partial length rods. The simulation of this step yields a more detailed prediction of the local effects of the thermal-hydraulic that will enhance the prediction of the Minimum CPR and will be used to locate the critical fuel pin.

5) The simulation of the previous step allows tracking the critical fuel pin according to the Minimum CPR criterion. Moreover, this figure of merit will be predicted in the corresponding axial location.

6) The last simulation step will use the boundary conditions of the fuel pin presenting the Minimum CPR. These boundary conditions will be loaded in a fuel behavior code FRAPCON/FRAPTRAN. The purpose is to account the effect of burnup distribution of the fuel rod that provides an axial distribution of the fuelcladding gap heat transfer coefficient. The feature will improve the prediction of the Minimum CPR.

7) Finally, the application of the methodology is complemented with the Uncertainty and Sensitivity analysis. This step analyzes input variables are relevant on the uncertainty of the prediction of the Minimum CPR. The uncertainty of the selected input variables is modelled and a sample of different cases coming from the perturbation of the selected input variables is run, for the last step of the proposed methodology in this application case. The result of running the sample yield the distribution of the output variable, i.e. the Minimum CPR. In this way, the boundaries of the uncertainty of the input variable is obtained, and used for evaluating the Safety Criteria.

The scheme of the steps undertaken and the flow of information is summarized and depicted in Figure 5.4. 


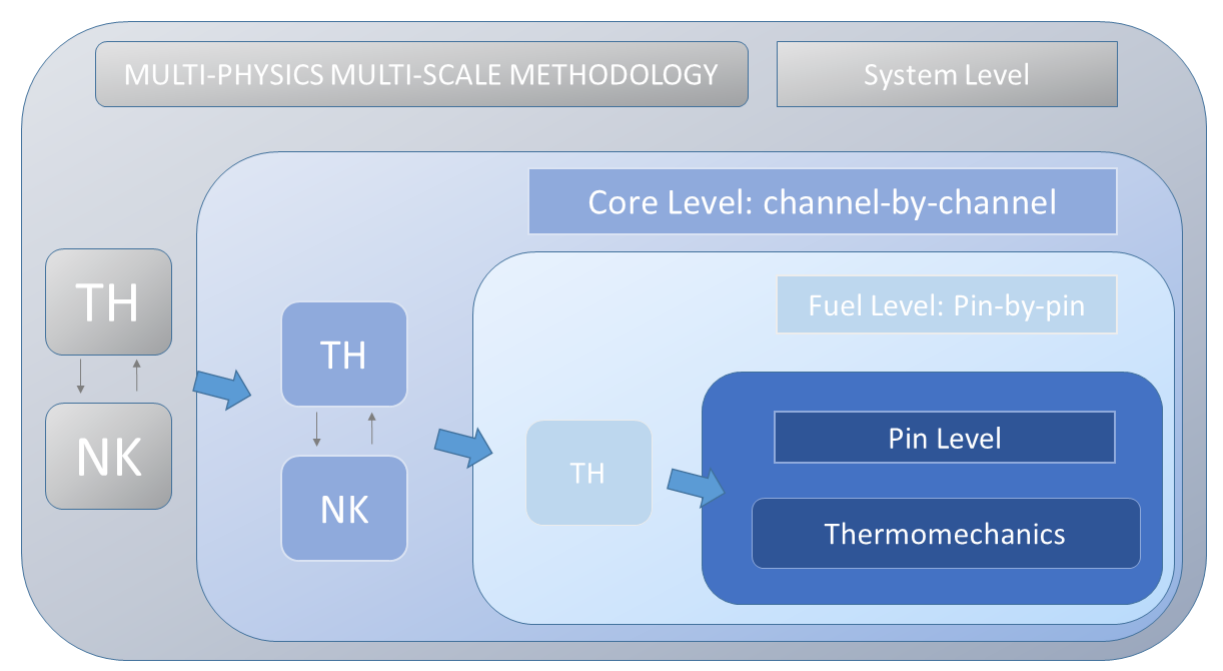

Figure 5.4 Scheme of information flow through the different steps of the proposed methodology.

\subsection{Discussion of results.}

This section explains the specific features of the step-by-step procedure of applying the methodology as proposed. Certain steps include the verification of the results in order to assure that the procedure is being undertaken in a coherent way. The verification of the corresponding steps is done by means of comparing the results with a reference code simulating the same scenario. Moreover, the results will be commented as needed.

\subsubsection{Verification of the cross-section libraries.}

The cross-section libraries are a key part of the simulation methodology. One of the most remarkable features of a Best Estimate analysis is to account the feedback between the $\mathrm{TH}$ and the NK and perform that interaction in 3D. For that purpose, a solid procedure for having the cross section data set at each node of the NK calculation is needed. The procedure applied in this step uses the SIMTAB interface. The results obtained in this step provide PARCS code with a 3D library that will be use to evaluate the cross-section sets at each node, according to the thermal-hydraulic variables and the control rod position for each time step of the simulation.

Therefore, the coherence of the cross-section variables must be verified against the reference code. To perform the code-to-code verification, three figures of merit are compared, being:

- Axial averaged power distribution, in the form of the percentage of Root Mean Square Error. 
- Radial averaged power distribution, in the form of the percentage of Root Mean Square Error.

- k-effective parameter compared as the absolute error measured in $\mathrm{pcm}$.

The comparison scenario is a steady state simulation of the 3D code modelled for PARCS. This model is equivalent to the one of SIMULATE-3. The PARCS model includes the 648 radial nodes representing the fuel channels, plus the radial reflector, resulting in 772 radial nodes. Each of the fuel channels are modelled with 25 axial active nodes plus top and bottom reflector hence, 27 nodes, each one of $0.1524 \mathrm{~m}$. Figure 5.5 depicts the nodal distribution of the reactor core.

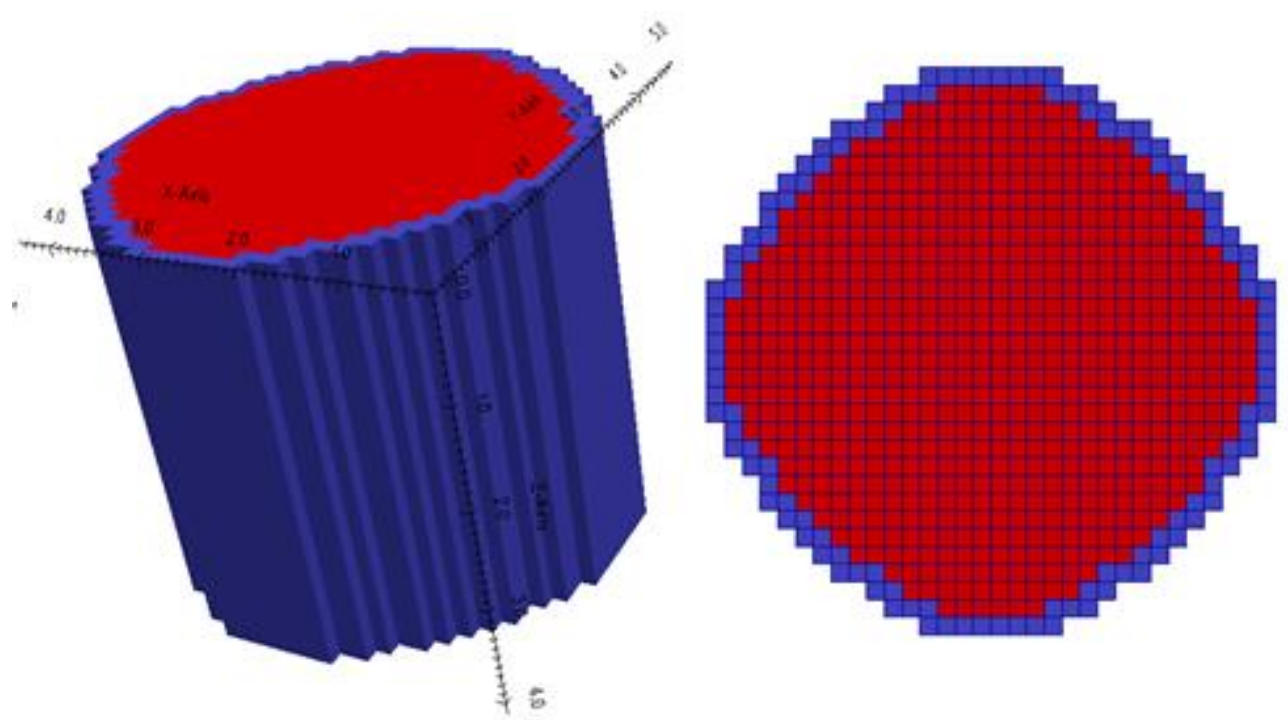

Figure 5.5 Nodal distribution of the 3D core model for PARCS.

The nemtab and nemtabr libraries correspond respectively to the uncontrolled and controlled cross section sets. For each of the compositions of the core. The distribution of the compositions is made and grouped according to the fuel channel segment and the burnup level. The active part of the model is compared to the results of SIMULATE-3. Figure 5.6 shows the comparison of the axial power distribution of PARCS and SIMULATE-3, Figure 5.6 shows the radial distribution of the relative error of the comparison of the radial power distribution of PARCS and SIMULATE-3. 
Development and validation of a multi-scale and multi-physics methodology for the safety analysis of fast transients in Light Water Reactors

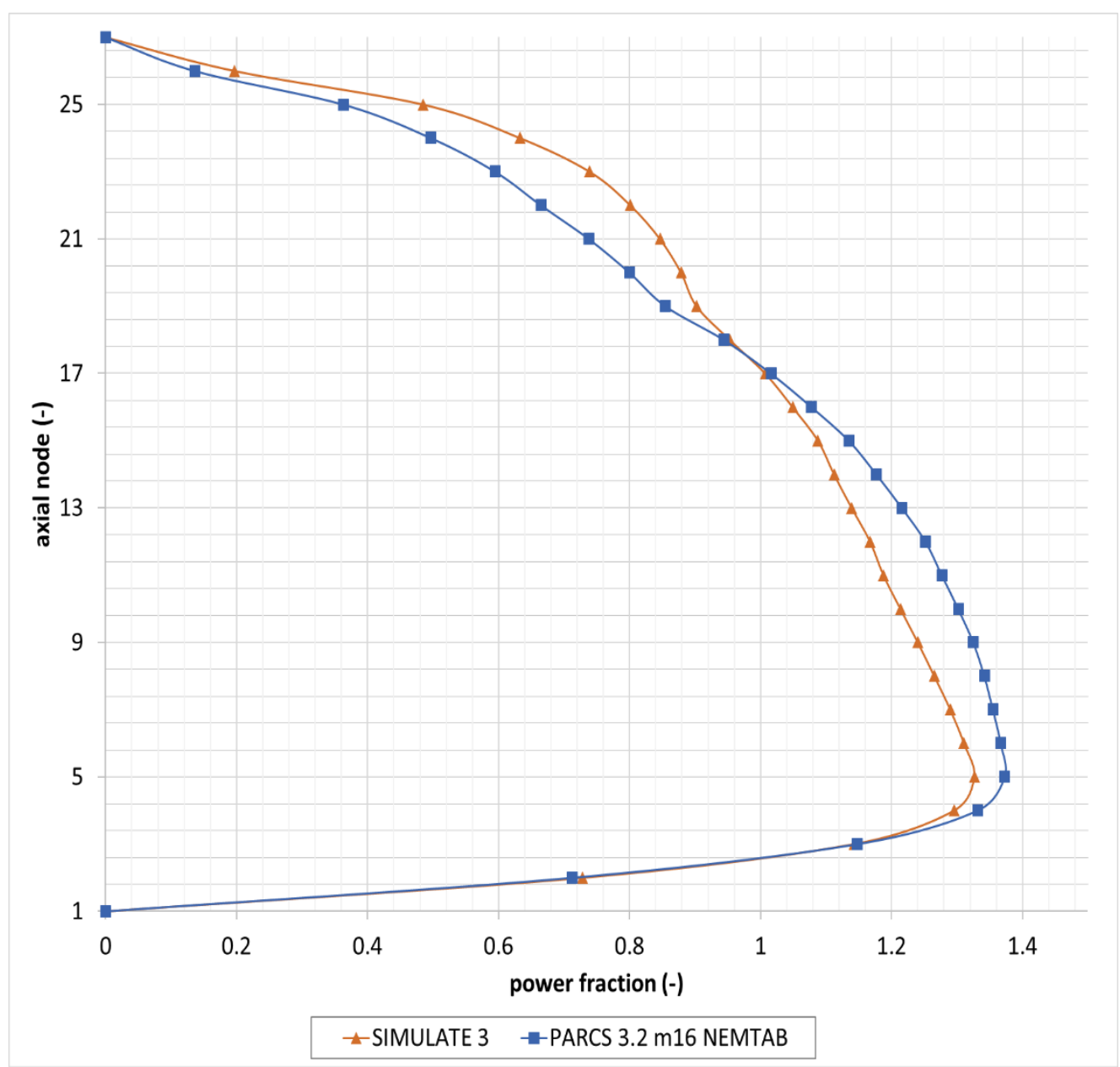

Figure 5.6 Comparison of the axial power profile of PARCS-NEMTAB and SIMULATE-3. 

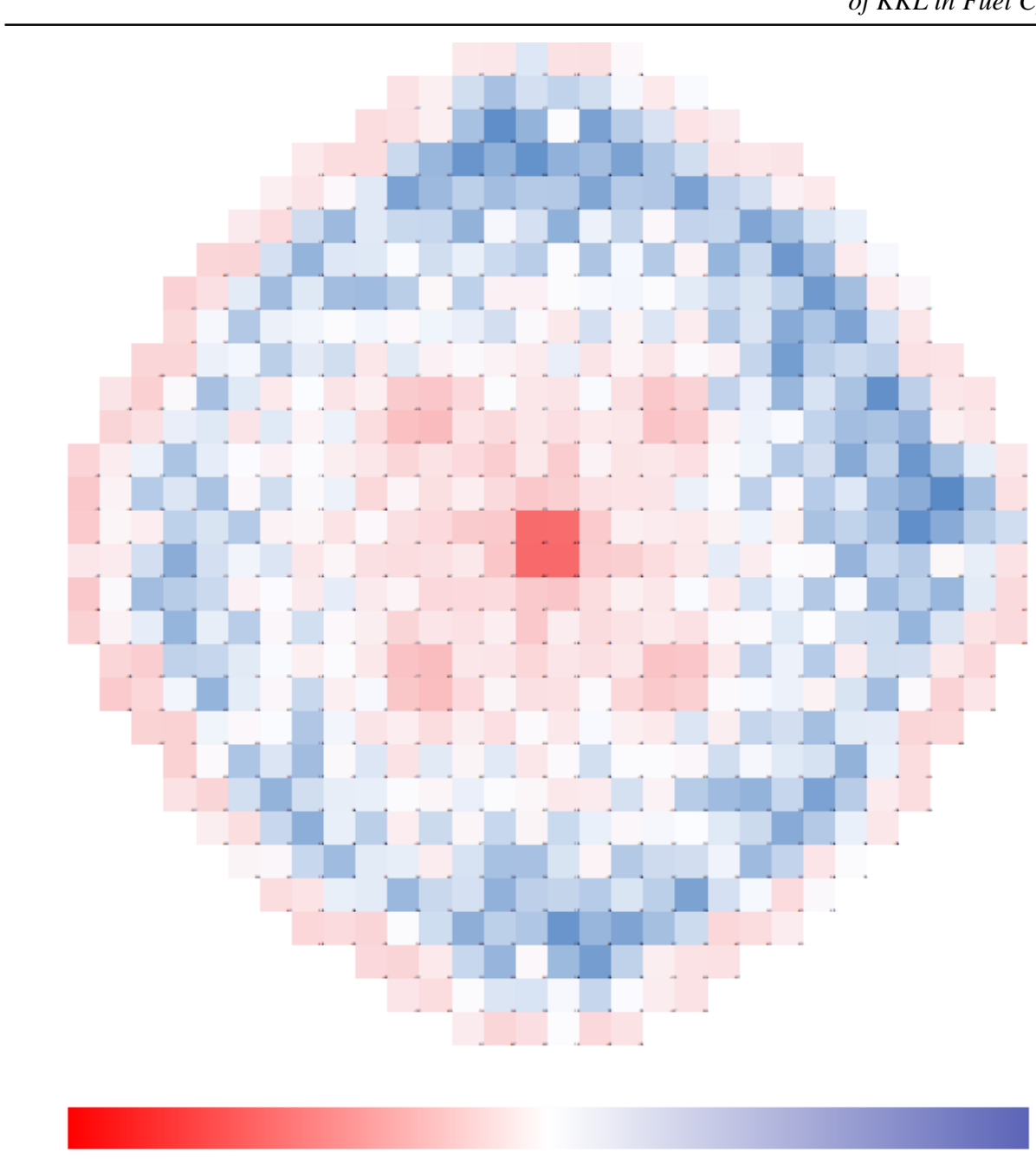

$-8.70 \%$

Relative Error (\%)

$6.45 \%$

Figure 5.7 Radial distribution of the relative error (\%) of the comparison PARCS (NEMTAB) vs SIMULATE-3.

The results of the comparison are summarized in Table 5.3. 
Development and validation of a multi-scale and multi-physics methodology for the safety analysis of fast transients in Light Water Reactors

Table 5.3 Summary of the results of the comparison PARCS-NEMTAB vs SIMULATE-3

\begin{tabular}{|l|l|l|l|}
\hline & $\begin{array}{l}\text { Axial Power } \\
\text { Profile } \\
\text { RMS Error } \\
(\boldsymbol{\%})\end{array}$ & $\begin{array}{l}\text { Radial Power Profile } \\
\text { RMS Error }(\boldsymbol{\%})\end{array}$ & $\begin{array}{l}\text { K-effective com- } \\
\text { parison (pcm) }\end{array}$ \\
\hline $\begin{array}{l}\text { Comparison } \\
\text { results }\end{array}$ & 7.93 & 4.03 & 378.1 \\
\hline
\end{tabular}

The results reveal the status of the SIMTAB interface for the generation of cross section libraries. The sources of error can be attached to two limitations to be reviewed. First of all, the incapability of SIMTAB to use more than one Control Rod composition, which forces to use the effect of the main control rod composition of the core. This leads to an error in the nodes where a different control rod composition is present as Figure 5.7 depicts. On the other hand, the effect of the assembly discontinuity factors must be reviewed, since there is an evident discrepancy in the symmetry of the radial power distribution between SIMULATE-3 and PARCS-NEMTAB. Nevertheless, the results are accepted for further steps.

\subsubsection{Simulation of the coupled thermal-hydraulic and neutron kinetic system model.}

The generation of the cross section libraries will be used in two steps, this first one uses a system model for the evaluation of the TH with the code TRACE. The calculation of the Neutron Diffusion equation in 3D will be undertaken by the PARCS code using the NEMTAB libraries.

The TRACE model used for the application of the proposed methodology is made of five main components. The recirculation lines are represented in one single loop. The rest of the components are modelled as depicted in Figure 5.8. 


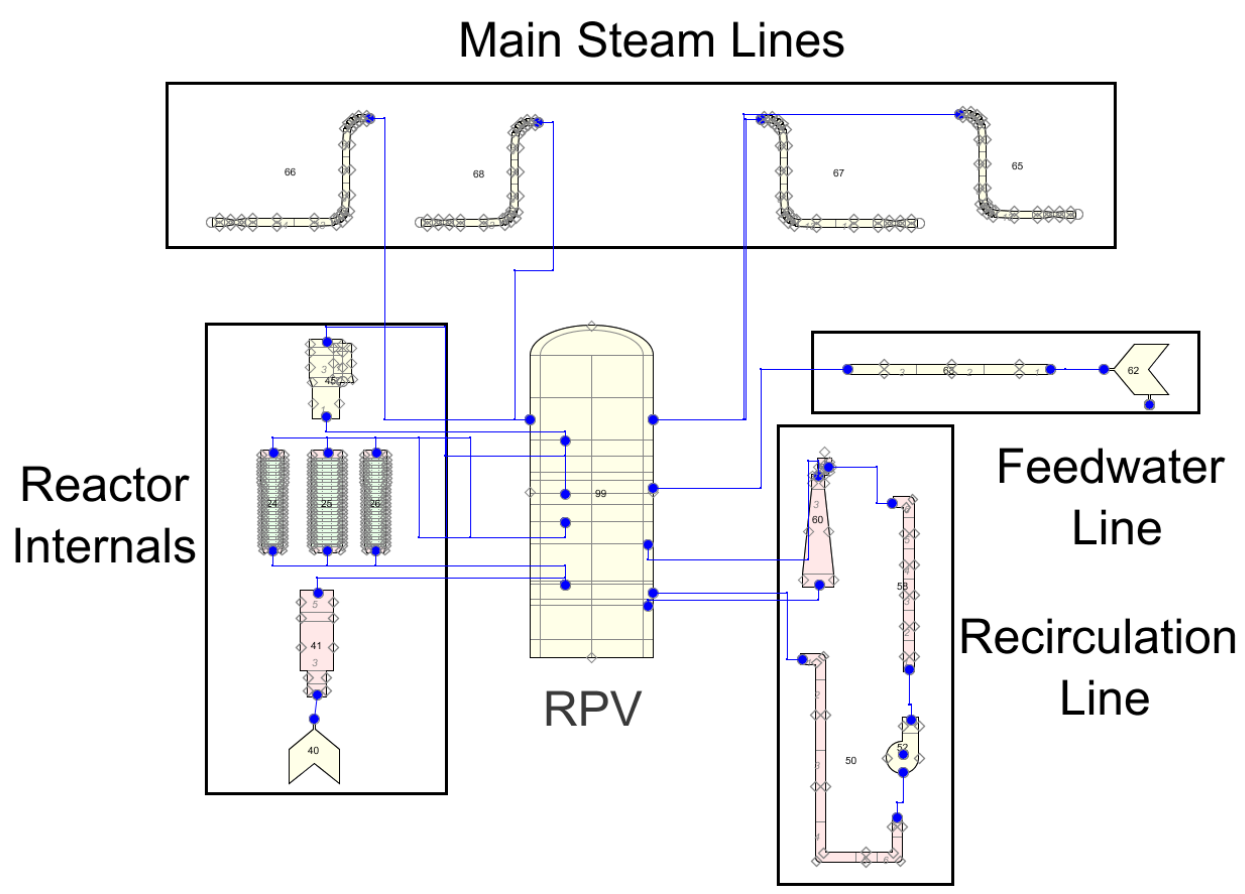

Figure 5.8 TRACE model scheme represented with SNAP.

The reactor pressure vessel models the BWR/6 of KKL lumping the 648 channels in three radial channels.

In this step, a converged solution of the steady-state of the TH is needed. This converged solution of the TH will be used to obtain the converged solution of the Coupled Steady State. And finally, this new converged solution will be used for simulating the coupled transient case i.e., the TT event. Therefore, the stand alone model of TRACE is run in order to generate a restart file for the Coupled Steady State. And afterwards, a new restart is obtained for the Transient case. The Stand Alone Steady State simulation was performed in 9955 time steps with a computer cost of 1819 seconds in a Linux machine with an Intel Core i7-3770 CPU at $3.40 \mathrm{GHz}$.

The coupled model is verified against the results of SIMULATE-3. Again, the figures of merit to be compared are the axial and radial power distribution and the k-effective parameter. The results of the code-to-code verification will be directly summarized in Table 5.4 . 
Development and validation of a multi-scale and multi-physics methodology for the safety analysis of fast transients in Light Water Reactors

Table 5.4 Summary of results of the code-to-code verification of TRACE/PARCS (NEMTAB) vs SIMULATE-3.

\begin{tabular}{|l|l|l|l|}
\hline & $\begin{array}{l}\text { Axial Power } \\
\text { Profile } \\
\text { RMS Error } \\
(\boldsymbol{\%})\end{array}$ & $\begin{array}{l}\text { Radial Power Profile } \\
\text { RMS Error }(\boldsymbol{\%})\end{array}$ & $\begin{array}{l}\text { K-effective compar- } \\
\text { ison }(\mathbf{p c m})\end{array}$ \\
\hline $\begin{array}{l}\text { Comparison } \\
\text { results }\end{array}$ & 15.10 & 6.83 & -201.70 \\
\hline
\end{tabular}

The results of Table 5.4 reveal the expected discrepancy between the thermal-hydraulic distribution of channels in SIMULATE-3 and the TRACE models. The reader must notice that the response of the $\mathrm{NK}$ to the feedback of $\mathrm{TH}$ is different in TRACE/PARCS(NEMTAB) than in SIMULATE-3. This is mainly to the fact that the reactivity feedback coefficients of the $\mathrm{TH}$ are to be averaged in three lumped channels for the case of TRACE/PARCS(NEMTAB) while in SIMULATE-3 the thermal-hydraulic model uses a channel-by-channel model. The consequence is the error rate in the power distribution while the comparison of the k-effective is kept in an acceptable margin, since the NK solution of the model uses a similar scheme.

The last part of this step simulates the transient case. The boundary conditions are introduced in the TRACE model i.e., valve maneuver and control rod movement. The figures of merit for the validation of this part of the methodology are the total core power and the dome pressure. The data has been gathered from KKL recording. Figure 5.9 and Figure 5.10 show the results of the validation for power and pressure respectively. 


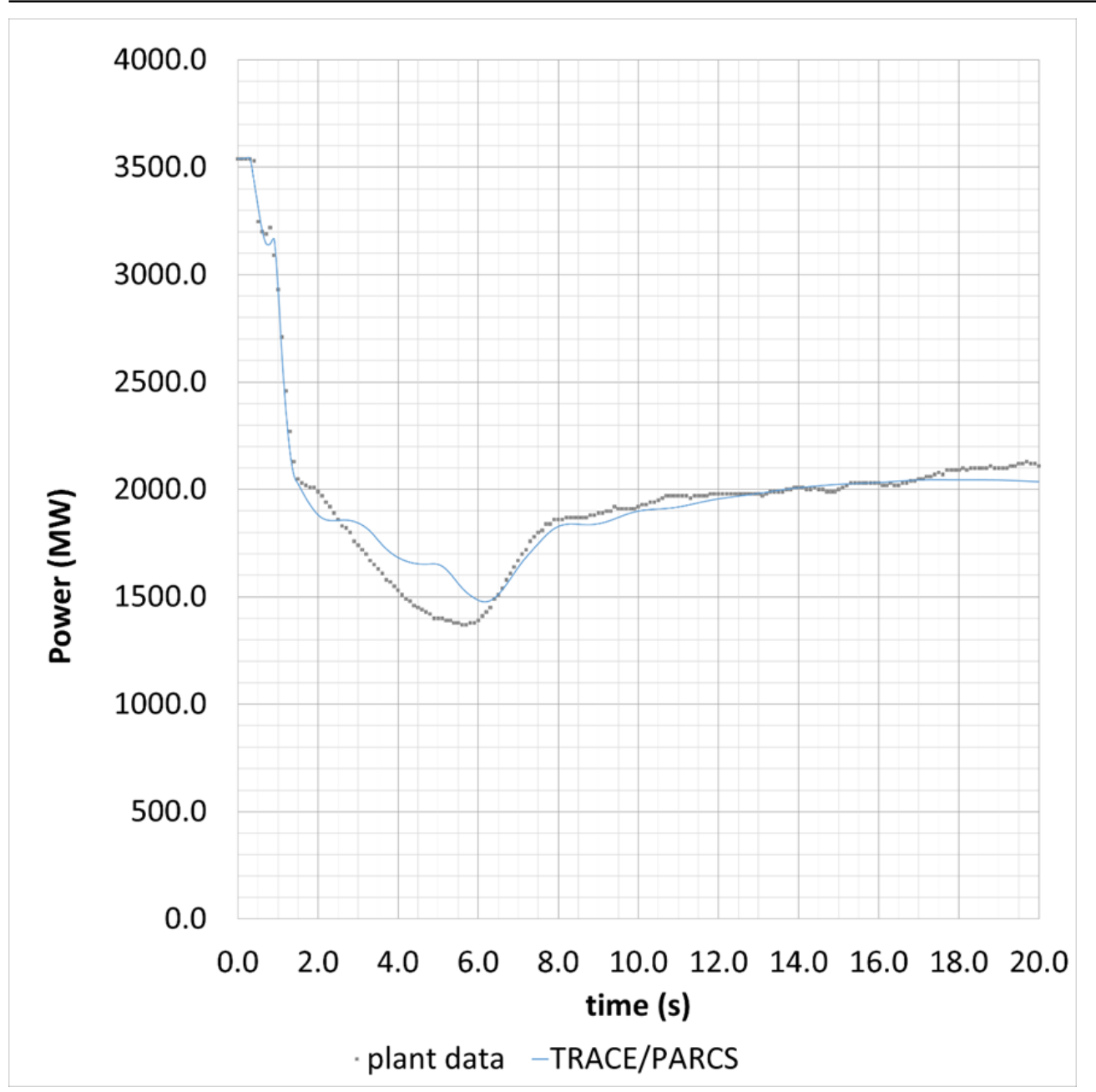

Figure 5.9 Total core power evolution: TRACE/PARCS(NEMTAB) vs Plant Data. 
Development and validation of a multi-scale and multi-physics methodology for the safety analysis of fast transients in Light Water Reactors

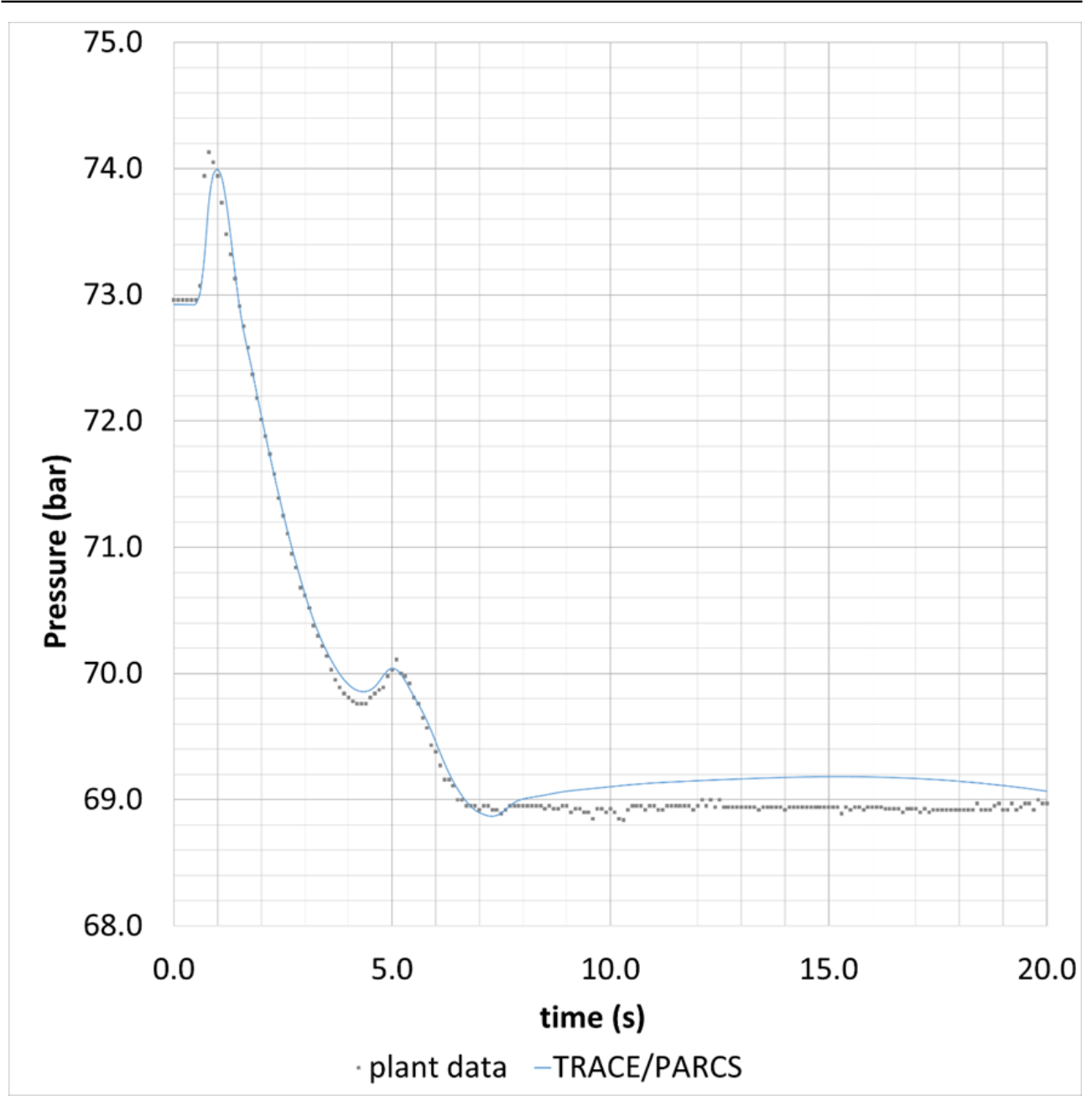

Figure 5.10 Dome pressure evolution: TRACE/PARCS vs Plant Data.

The results show good agreement on the transient response due to the feedback of the $\mathrm{TH}$ and the NK. The evolution of the mass flow in the core is modelled by the TRACE model by means of valves maneuver and the power is controlled with the implemented Selected Rod Insertion.

The simulation of this step of the methodology yields the boundary conditions of the core in regard of the predicted pressure evolution and inlet mass flow. These data will be used as boundary conditions in the next step, where the reactor core is being modelled in a more detailed scale. This enhancement in the scale of the model will allow a local 
prediction of the Minimum CPR that, instead of accounting a core averaged level will allow tracking the critical fuel channel.

\subsubsection{Simulation of the coupled TH and NK core model defined channel-by-channel.}

The results of the previous step are loaded as boundary conditions for simulated a channel-by-channel core model using the coupled scheme CTF-UPVIS and PARCS. The PARCS model corresponds to the same model as the previous one. The CTF-UPVIS model includes 25 axial nodes for the active part of the fuel as well as the top and bottom reflector nodes. Moreover, the radial distribution is made defining the 648 fuel channel nodes, plus an external channel that is used as bypass.

For the definition of this model, the specific geometry of different fuel types is used. During the development of the thesis work different fuel assembly models where defined with a subchannel scheme. Each of the fuel assemblies account detailed geometrical design that considers water rods and partial length rods.

The channel-by-channel model lumps the geometry of each fuel pin-by-pin model in the corresponding flow area and wetted perimeter. Therefore, the detail of the level of the CTF-UPVIS model is enhanced in regard of the TRACE core model.

The aim of this step of the simulation methodology is to track the specific fuel channel where the Minimum CPR is predicted. For that purpose, as for the TRACE/PARCS simulation process, three substeps must be undertaken: the convergence of the Steady State Stand Alone solution with CTF-UPVIS, the converged solution of the coupled Steady State, and the coupled transient case simulation.

The reader must notice that the cross-section libraries have been already validated, therefore, in this section only the results of the verification of the coupled CTFUPVIS/PARCS steady state will be presented. Table 5.5 shows the results of the codeto-code verification of the steady state of SIMULATE-3 and CTF-UPVIS/PARCS.

Table 5.5 Summary of results of the code-to-code verification of CTF-UPVIS/PARCS (NEMTAB) vs SIMULATE-3.

\begin{tabular}{|l|l|l|l|}
\hline & $\begin{array}{l}\text { Axial Power } \\
\text { Profile } \\
\text { RMS Error } \\
(\boldsymbol{\%})\end{array}$ & $\begin{array}{l}\text { Radial Power Profile } \\
\text { RMS Error }(\boldsymbol{\%})\end{array}$ & $\begin{array}{l}\text { K-effective compar- } \\
\text { ison (pcm) }\end{array}$ \\
\hline $\begin{array}{l}\text { Comparison } \\
\text { results }\end{array}$ & 13.60 & 3.44 & 129.00 \\
\hline
\end{tabular}

In view of the results of Table 5.5, the radial error has been reduced respect to the results of TRACE/PARCS since the CTF/PARCS model is equivalent to SIMULATE-3 
Development and validation of a multi-scale and multi-physics methodology for the safety analysis of fast transients in Light Water Reactors

scheme, i.e. a channel-by-channel model for the TH. The error of the k-effective parameter shows good agreement since both codes of the comparison use the same source for the calculation of the NK.

Once the code-to-code verification is done, the next step is validating the transient results against plant data. The results to be compared are the evolution of the core power, since the pressure of the core is a forced boundary condition as well as the inlet mass flow. Figure 5.11 shows the evolution of the power of the CTF-UPVIS/PARCS model in addition to the previous results of TRACE/PARCS in order to complement the code-tocode verification of TRACE/PARCS and CTF-UPVIS/PARCS.

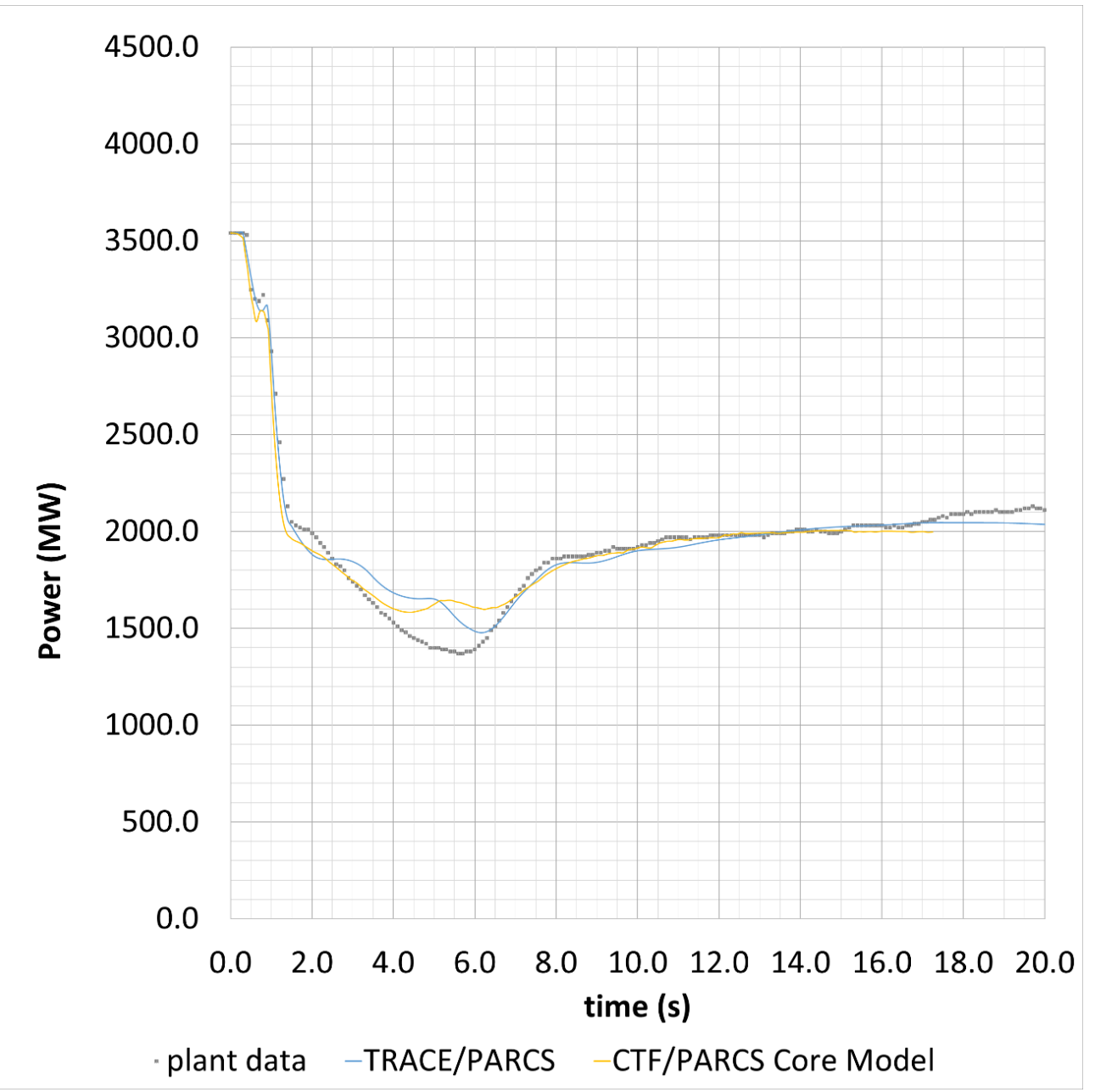

Figure 5.11 Reactor core evolution of the CTF-UPVIS/PARCS model and TRACE/PARCS model against plant data. 
Chapter 5. Application of the proposed methodology to the Safety Analysis of the Turbine Trip Event of KKL in Fuel Cycle 18

In view of the results, the channel-by-channel core model defined with CTFUPVIS/PARCS shows good agreement compared to the TRACE/PARCS results and the plant data. In addition, this step of the proposed methodology allows tracking the fuel channel having the Minimum CPR. Therefore, the presented methodology gives a better evaluation of such a local safety variable as the Minimum CPR.

Figure 5.12 shows the evolution of the Minimum CPR of the most critical channels i.e., the fuel channels conforming the envelope of the Minimum CPR. This means that the Minimum CPR along the transient is defined by one of the channel Minimum CPR depending on the transient time step.

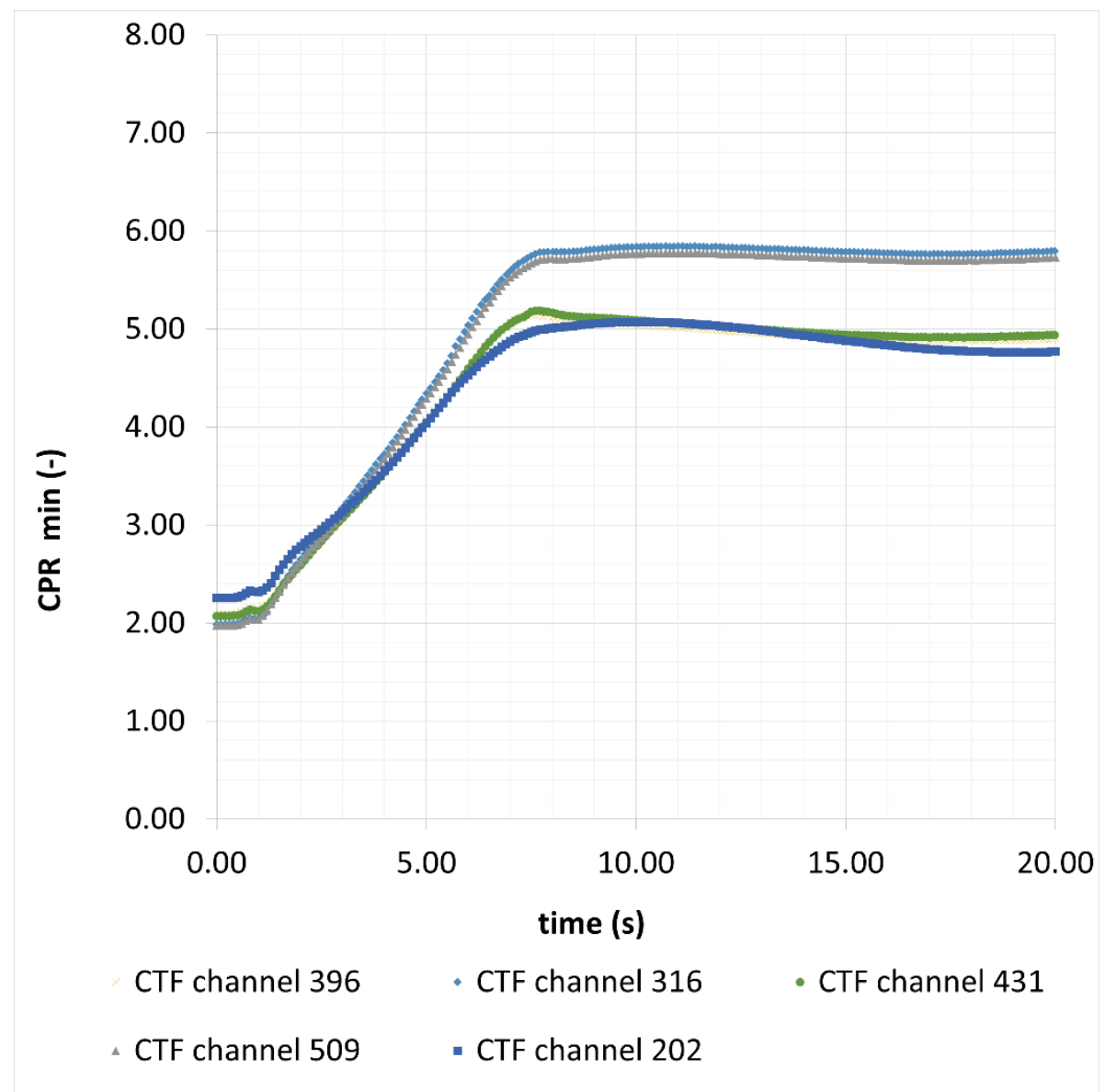

Figure 5.12 Evolution of the Minimum CPR of the fuel critical fuel channels retrieved from the simulation of the CTF-UPVIS/PARCS core model. 
Development and validation of a multi-scale and multi-physics methodology for the safety analysis of fast transients in Light Water Reactors

Moreover, the Figure 5.13 shows the core map where the critical fuel channels can be located. Noticed that in a lumped core model in 3 channels like the TRACE/PARCS model this approach could not have been obtained.

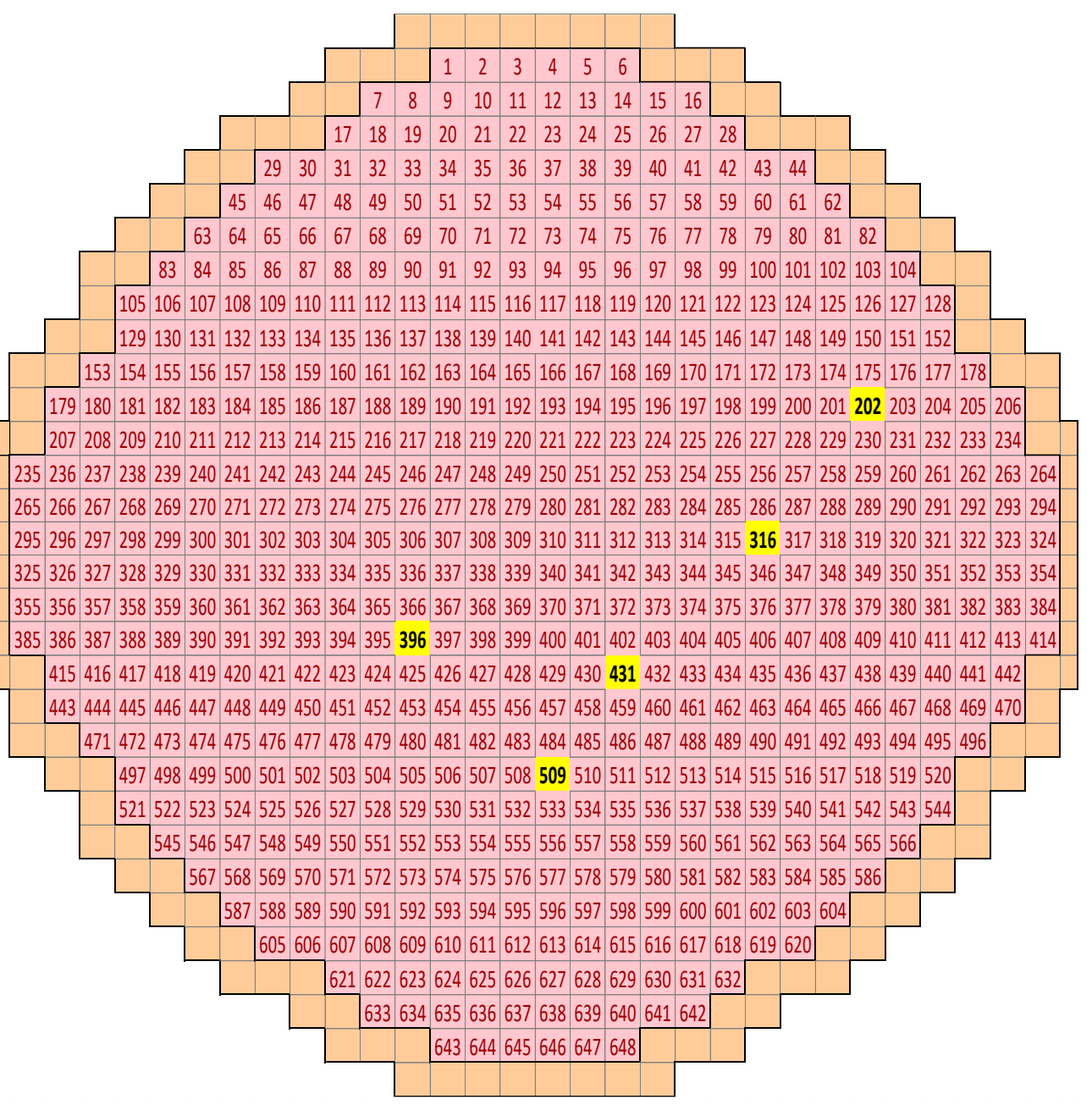

Figure 5.13 core map highlighting the critical fuel channels according to the Minimum CPR.

As Figure 5.12 shows the Minimum CPR is located at the beginning of the transient. Table 5.6 shows the Minimum CPR achieved in each of the selected critical fuel channels. 
Table 5.6 Minimum CPR for each of the fuel critical fuel channels.

\begin{tabular}{|c|c|}
\hline Channel & Minimum CPR (-) \\
\hline $\mathbf{5 0 9}$ & 1.97 \\
\hline $\mathbf{3 1 6}$ & 1.99 \\
\hline $\mathbf{4 1 3}$ & 2.07 \\
\hline $\mathbf{3 9 6}$ & 2.08 \\
\hline $\mathbf{2 0 2}$ & 2.25 \\
\hline
\end{tabular}

Channels 509 and 316 experiment similar Minimum CPR at the beginning of the transient. Due to this, the critical case is assumed for channel 509 that will be implemented in the following steps of this methodology.

The proposed multi-scale and multi-physics methodology is applied only for one single fuel channel. The fuel channel selected is the 509 due to the aforementioned reasons. In the following subsection the next step is explained and simulated. A more extended analysis would consider applying this methodology to every fuel channel that has endured the Minimum CPR. Table 5.6 shows very small difference between the Minimum CPR of the critical channels (specially for channels 509 and 316) and a more detailed scale analyzed with a BE tool such as CTF-UPVIS can reveal the Minimum CPR in other fuel channel other that 509 if such small differences exist.

The application of the proposed methodology goes one step further performing the simulation of the transient in a more local scale. For that purpose, a pin-by-pin fuel model will be used. In that model, the critical fuel rod will be tracked in regard of the Minimum CPR criterion.

\subsubsection{Simulation of the subchannel thermal-hydraulic model.}

This next step of the presented methodology uses a pin-by-pin fuel model adding the detail components such as water rods and partial length rods. The transient is simulated with a CTF-UPVIS subchannel model where the boundary conditions of the transient have been loaded according to the fuel channel data retrieved from the previous step of the methodology i.e., the CTF-UPVIS/PARCS core model.

The pin-by-pin model is selected from a library of fuel models that has to be available and previously designed to be ready to provide the detailed fuel model selected as critical from the core model simulation. 
Development and validation of a multi-scale and multi-physics methodology for the safety analysis of fast transients in Light Water Reactors

According to the results of the core simulation, fuel channel 509 is located in the library of CTF-UPVIS subchannel models. The fuel model corresponds to a fuel assembly with a central water rod and 4 water wings that subdivide the assembly in 4 sub-bundles. Moreover, the fuel assembly has two partial length rods of $1 / 3$ and $2 / 3$ the full length of a regular rod.

Figure 5.14 and Figure 5.15 show the layouts of the radial distribution of the fuel model to be analyzed and the axial nodal distribution.

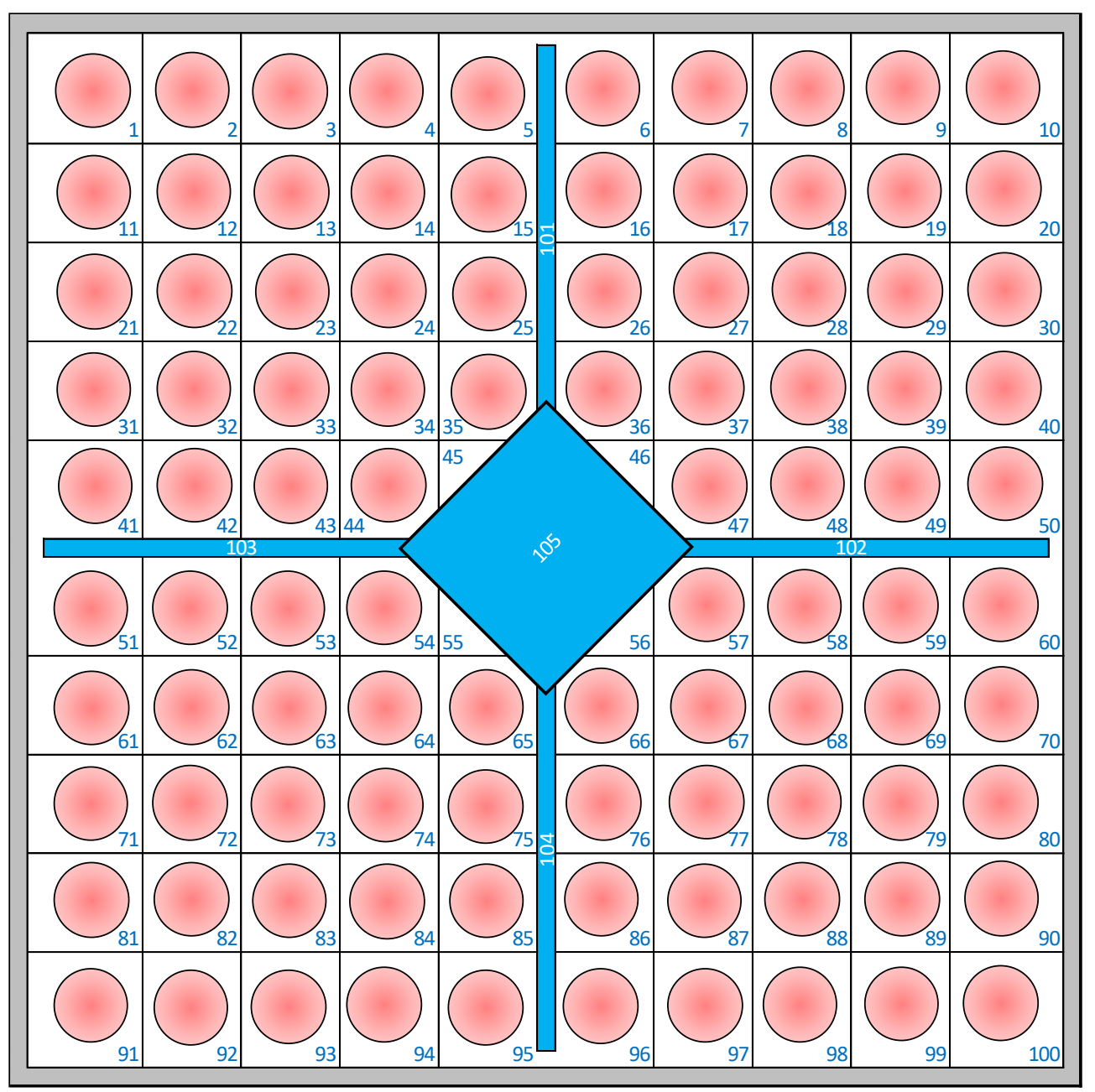

Figure 5.14 Radial nodal distribution of fuel channel 509. 


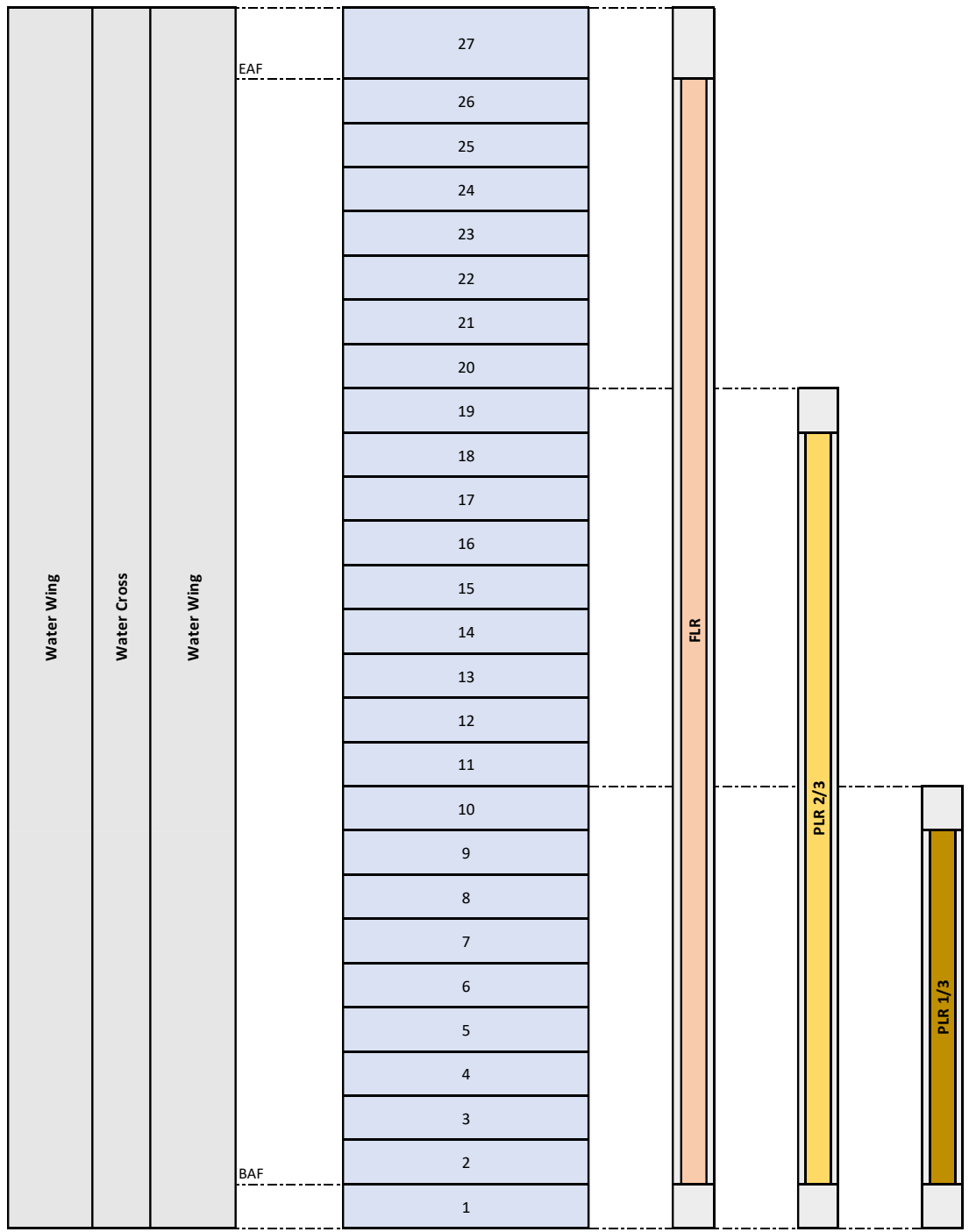

Figure 5.15 Axial nodal distribution of the fuel channel 509 with the detail of the partial length rods.

As Figure 5.14 depicts, there are 100 subchannels centered on the fuel rod. In addition, there are 4 subchannels for the water wings and 1 subchannel for the central water rod. Moreover, Figure 5.15 shows that the axial distribution has been designed using 25 active nodes plus one bottom and top node being all of them of $0.1524 \mathrm{~m}$. 
Development and validation of a multi-scale and multi-physics methodology for the safety analysis of fast transients in Light Water Reactors

As commented the boundary conditions are retrieved from fuel channel 509 using a MATLAB based application that loads the corresponding forcing functions of inlet enthalpy of the coolant, outlet pressure, inlet mass flow and power evolution. The result of the simulation of the subchannel fuel model gives the averaged prediction of the Minimum CPR. Figure 5.16 shows the comparison of the fuel channel of the core model of CTF-UPVIS/PARCS and the averaged fuel subchannel model.

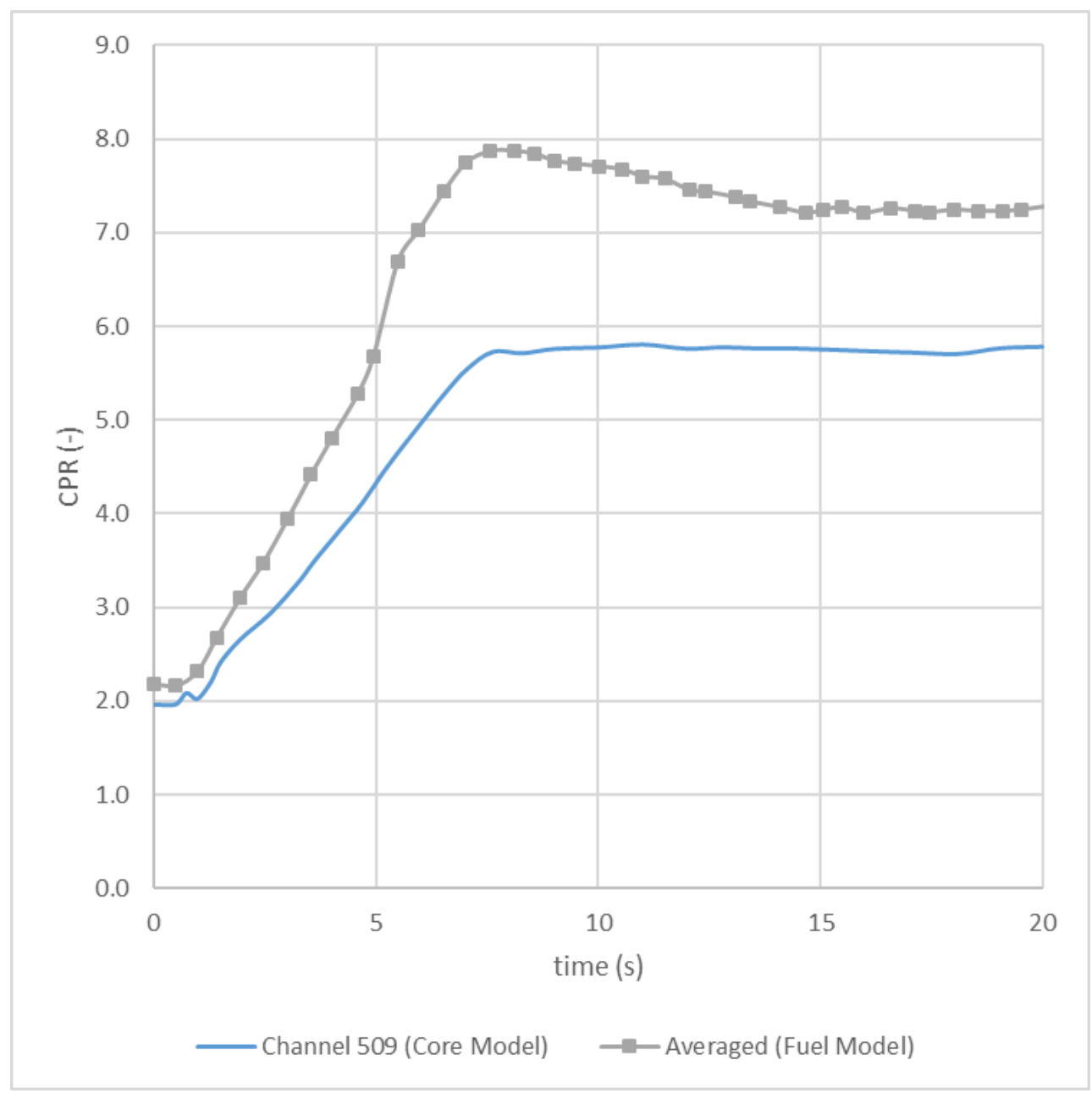

Figure 5.16 Comparison of the prediction of the Minimum CPR between the fuel channel 509 of the CTF-UPVIS/PARCS core model and the average pin-by-pin fuel model of CTF-UPVIS.

As Figure 5.16 shows, the use of a more detailed model gives a higher prediction of the Minimum CPR. The difference of the prediction of the Minimum CPR is related to the boundary conditions of the fuel suchannel model of CTF-UPVIS. In the core model of 
Chapter 5. Application of the proposed methodology to the Safety Analysis of the Turbine Trip Event of KKL in Fuel Cycle 18

CTF-UPVIS/PARCS simulation, channel 509 accounts the heat transfer between the adjacent fuel channels, whilst the subchannel of CTF-UPVIS uses an adiabatic boundary condition for the canister wall. This difference of the approach is solved adding the corresponding boundary conditions of the heat transfer to the canister wall of the CTFUPVIS model. Nevertheless, the purpose of simulating a pin-by-pin fuel model is to evaluate at the pin level the prediction of the Minimum CPR.

In this next step of the proposed methodology, the pin-by-pin model is used to locate the critical fuel pin according to the Minimum CPR. The pin-by-pin model can show the different approaches that this methodology can provide. First of all, the average value of the model gives a value of the Minimum CPR evolution, with a lower level of detail due to the coarse mesh. Secondly, a more conservative approach can be done by retrieving the envelope of the Minimum CPR that is predicted along all the fuel rods during the full time of the simulation. Finally, the Best Estimate approach gives the fuel rod with Minimum CPR and its axial location. The realistic prediction of this local phenomenon is analyzing the location where the Minimum CPR is predicted during the full length of the transient.

Figure 5.17 shows the evolution of the Minimum CPR according to the three different approaches. Moreover, Figure 5.17 shows at each time step the axial location of the Minimum CPR for the different approaches.

The analysis shows that the Minimum CPR is located in fuel rod 12 at the beginning of the transient in axial position 19. The envelope prediction fits with the critical prediction until second 5, where the Minimum CPR changes to rod 2 until second 16, and finally changes to rod 11. During the full simulation of the transient, the Minimum CPR is located at axial node 19 for the envelope prediction, and varies the axial position. The average value predicts the Minimum CPR at node 17. In this case, it could be assumed that the average prediction of the Minimum CPR is more conservative since it is located in a lower position. This is due to the critical combination of parameters predicting the Minimum CPR has an onset lower than the envelope and the evolution of the critical fuel rod. In this case, the simulation of the pin-by-pin fuel model avoid a conservative prediction. 
Development and validation of a multi-scale and multi-physics methodology for the safety analysis of fast transients in Light Water Reactors

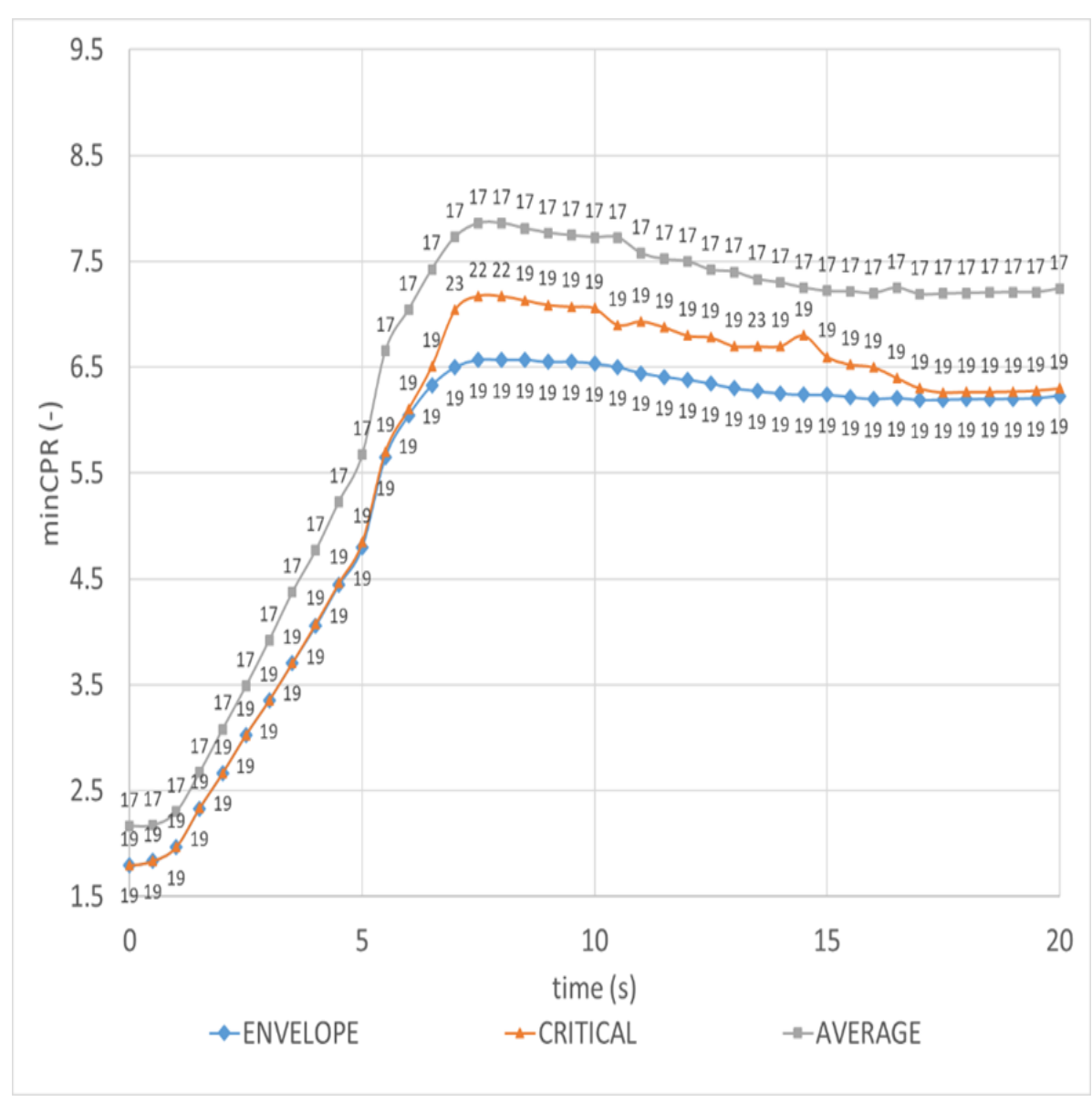

Figure 5.17 Evolution of the Minimum CPR with the approaches of the average value, the envelope and the critical fuel rod.

\subsubsection{Pin analysis including the fuel behavior simulation.}

In this subsection, the analysis uses the fuel behavior code FRAPCON/FRAPTRAN. The boundary conditions of fuel rod 12 are loaded in a fuel pin model. The target is to add the thermo-mechanics of FRAPCON/FRAPTRAN to the calculations of CTFUPVIS. Notice that FRAPCON/FRAPTRAN calculates the fuel behavior of the fuel pin from the centerline of the fuel pellet to the outer diameter of the cladding, which is a more detailed approach than the one made by CTF-UPVIS. Conversely, the TH are assumed in a simpler way. Therefore, adding the capabilities of FRAPCON/FRAPTRAN to the pin simulation of the TT it is possible to have a Best Estimate approach. 
Chapter 5. Application of the proposed methodology to the Safety Analysis of the Turbine Trip Event of KKL in Fuel Cycle 18

Moreover, FRAPCON/FRAPTRAN allows the definition of the axial distribution of the heat transfer coefficient of the fuel-cladding gap, instead of assuming a uniform heat transfer coefficient.

FRAPCON is a fuel behavior code for steady-state calculations, where the evolution of the fuel behavior is obtained by introducing a time dependent evolution of the operation conditions of the fuel rod. A restart file is generated and used in the simulation of FRAPTRAN. In this manner, it is possible to obtain the aforementioned axial distribution of the gas-gap heat transfer coefficient.

The use of FRAPCON/FRAPTRAN allows obtaining the evolution of the Minimum CPR of the critical axial position, i.e. where the Minimum CPR of the transient is located. Moreover, it is possible to obtain the envelope of the Minimum CPR, i.e. the different positions of the Minimum CPR in the fuel pin along the duration of the transient.

Figure 5.18 shows the evolution of the prediction of the Minimum CPR of the critical fuel pin with CTF-UPVIS, the critical axial location, corresponding to axial node 15 and the envelope, both with FRAPCON/FRAPTRAN.

In view of the results of Figure 5.18, FRAPCON/FRAPTRAN predicts a lower Minimum CPR than the critical prediction of CTF-UPVIS. Accounting the heat transfer coefficient of the gas-gap adds a more accurate prediction of the surface heat flux, which is relevant for the calculation of the Minimum CPR. Therefore, incrementing the known physics in the methodology modifies the results having a Best Estimate calculation.

Nevertheless, such Best Estimate approach needs of the corresponding uncertainty and sensitivity calculations, in order to define the boundaries of the uncertainty in the prediction of the safety variable, in this case the Minimum CPR. 
Development and validation of a multi-scale and multi-physics methodology for the safety analysis of fast transients in Light Water Reactors

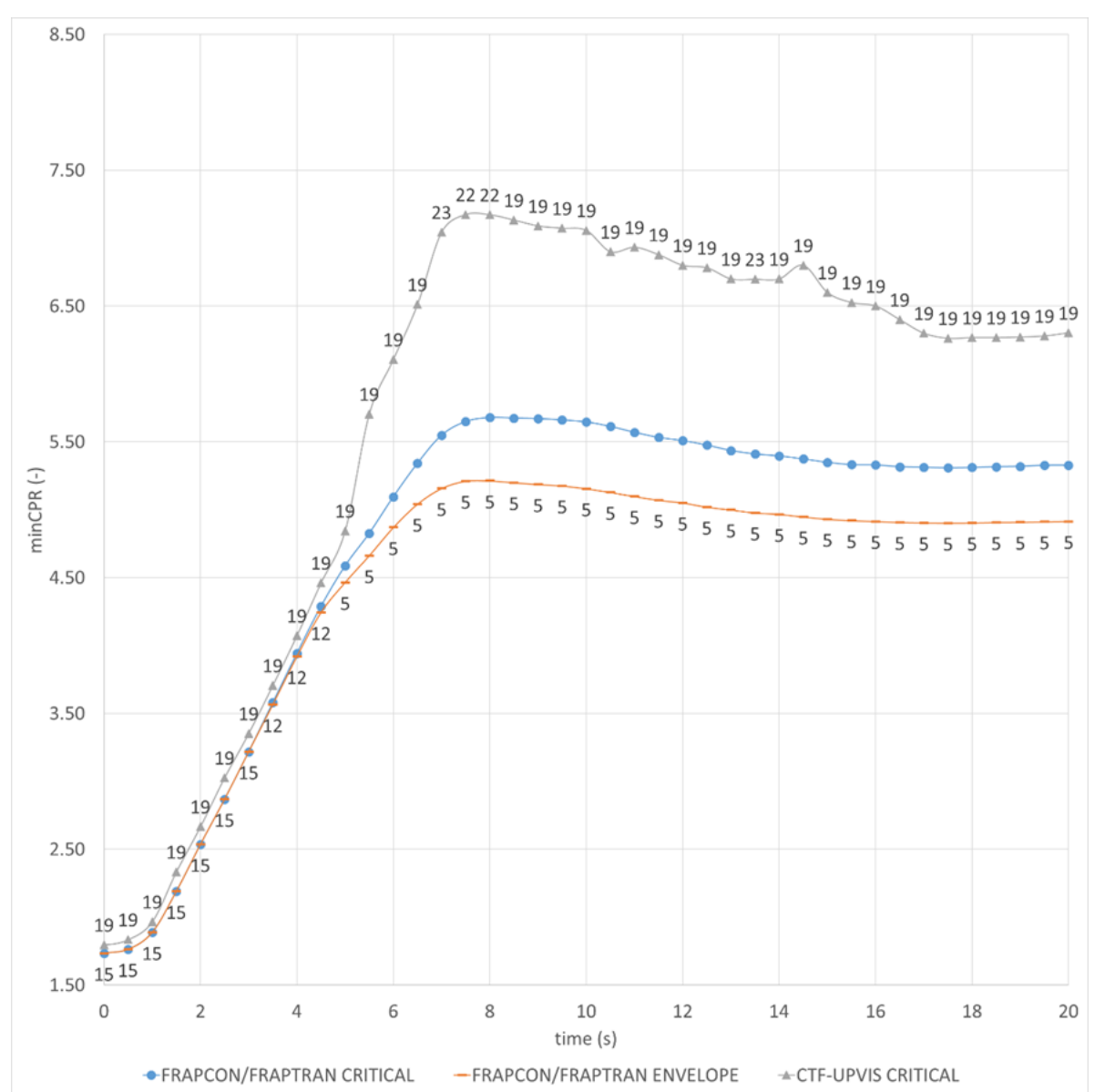

Figure 5.18 Prediction of the Minimum CPR by FRAPCON/FRAPTRAN pin model compared with the prediction of the CTF-UPVIS pin model.

\subsubsection{Uncertainty and sensitivity analysis.}

The Uncertainty Quantification has the aim of complementing this simulation methodology. The purpose is to define the limits of the predicted variable according to certain statistic criteria. Therefore, the propagation of uncertainties coming from input variables is accounted in the evaluation of the output target variable by means of the SA. The approach reported in this paper will use the scientific literature to account the uncertainty of the most relevant variables affecting the uncertainty of the prediction of the Minimum CPR. The final result of the methodology is to evaluate the upper and lower boundaries of the prediction of the Minimum CPR and which is the probability of the simulation results to obtain a result in such Confidence Interval. 
The features of this step include the DAKOTA toolkit, the scientific literature (National Technology and Engineering Solutions of Sandia, 2017) in order to realize an appropriate Uncertainty Quantification, and the Wilks theory (Wilks, 1941). The Wilks theory is used to set the sample size of the needed simulations. The method is based on introducing perturbations in the selected input variables, according to their PDFs. This set of perturbations generate a certain number of simulations to be run, i.e. the size of the sample. For the case presented in this report, the sample size is of 146, according to Wilks Formula. This sample size is derived from the selected Statistic Criterion, namely 95/95. This criterion defines that the $95 \%$ of the cases of the sample will fall into a Confidence Interval of $95 \%$. This criterion is sufficient to accomplish the acceptance criteria of the Nuclear Authority. According to the scientific literature, Table 5.7 shows the selected input variables that are assumed to introduce uncertainty in the prediction of the Minimum CPR.

Table 5.7 Sources of uncertainty considered.

\begin{tabular}{|l|c|c|c|c|}
\hline \multicolumn{1}{|c|}{ parameter } & units & mean & $\begin{array}{c}\text { Std. } \\
\text { Dev./bound- } \\
\text { ary }\end{array}$ & PDF \\
\hline $\begin{array}{l}\text { Cladding outer } \\
\text { diameter }\end{array}$ & $(\mathrm{m})$ & 0.009500 & 0.000019 & Normal \\
\hline $\begin{array}{l}\text { Cladding inner } \\
\text { diameter }\end{array}$ & $(\mathrm{m})$ & 0.008357 & 0.000019 & Normal \\
\hline $\begin{array}{l}\text { Pellet dish ra- } \\
\text { dius }\end{array}$ & $(\mathrm{m})$ & 0.002475 & 0.000063 & Normal \\
\hline Fuel density & $(\%)$ & 95.50000 & 0.750000 & Normal \\
\hline Pellet diameter & $(\mathrm{m})$ & 0.008192 & 0.000006 & Normal \\
\hline $\begin{array}{l}\text { Cladding rough- } \\
\text { ness }\end{array}$ & $(\mu \mathrm{m})$ & 0.635500 & 0.317250 & Normal \\
\hline Fuel roughness & $(\mu \mathrm{m})$ & 1.600500 & 0.799750 & Normal \\
\hline Plenum length & $(\mathrm{m})$ & 0.029531 & 0.000884 & Normal \\
\hline Outlet pressure & $(\mathrm{bar})$ & 73.64400 & 0.010000 & Normal \\
\hline Inlet mass flow & $(\mathrm{kg} / \mathrm{s})$ & 0.113807 & 0.010000 & Normal \\
\hline $\begin{array}{l}\text { Inlet tempera- } \\
\text { ture }\end{array}$ & $(\mathrm{K})$ & 550.6200 & \pm 0.01000 & Uniform \\
\hline
\end{tabular}


Development and validation of a multi-scale and multi-physics methodology for the safety analysis of fast transients in Light Water Reactors

The results are obtained using a MATLAB-based interface that pre-processes the statistical distribution of the target input variables in order to generate the input for DAKOTA. Afterwards, DAKOTA generates the 146 cases for FRAPTRAN. Once the simulation of the 146 cases is done, DAKOTA realizes the post-processing retrieving the statistics of the Uncertainty and Sensitivity analysis. Figure 5.19 shows the definition of the Confidence Interval (CI), the mean value of the Uncertainty Quantification, and the result of the nominal value with the pin model of FRAPTRAN.

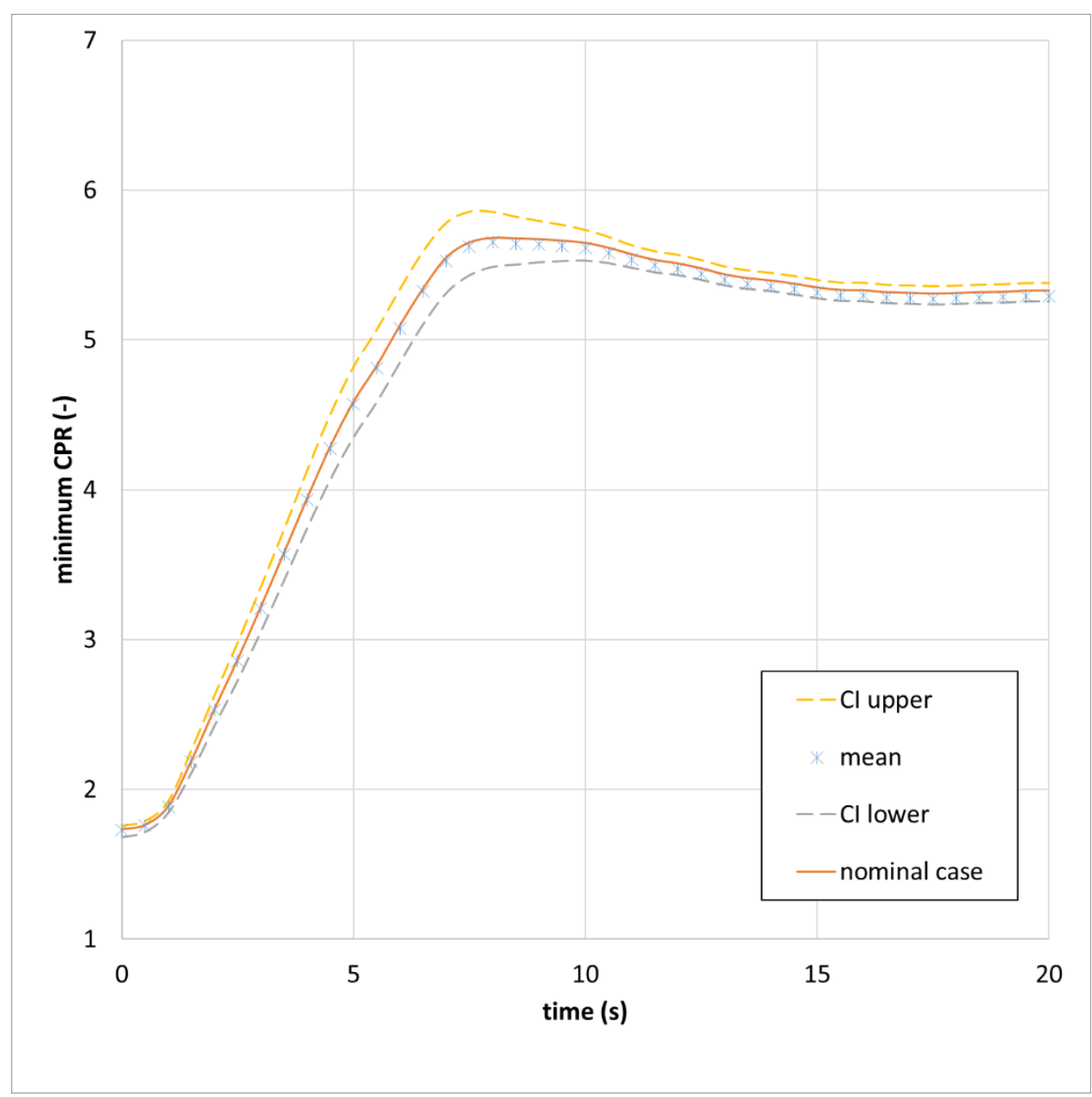

Figure 5.19 Statistics results of the Minimum CPR prediction with FRAPTRAN pin model.

As it can be expected, the nominal value of the simulation matches with the mean value of the sample. This step of the methodology defines the statistical boundaries with the 95/95 criterion. The results of the Uncertainty Quantification revealed that for each time 
Chapter 5. Application of the proposed methodology to the Safety Analysis of the Turbine Trip Event of KKL in Fuel Cycle 18

step the results of the 146 cases fell into the CI with a probability between 99.32 and 95.89 per cent. Therefore, the $95 / 95$ criterion is met.

In addition, the DAKOTA toolkit has the feature of defining the Sensitivity Analysis. This analysis reveals how the uncertainty of the Minimum CPR (as target output variable) is sensitive to the variations of the selected input variables. The procedure is made by analyzing the correlation between the input variables and the output variable. For this purpose, this simulation methodology uses the Partial Rank Correlation Coefficients (PRCC). It is assumed that values of PRCC above 0.20 in absolute value reveal significant effect of the uncertainty of the input in the uncertainty of the output. Figure 5.20 shows the result of the Sensitivity Analysis for Table 5.8 variables.

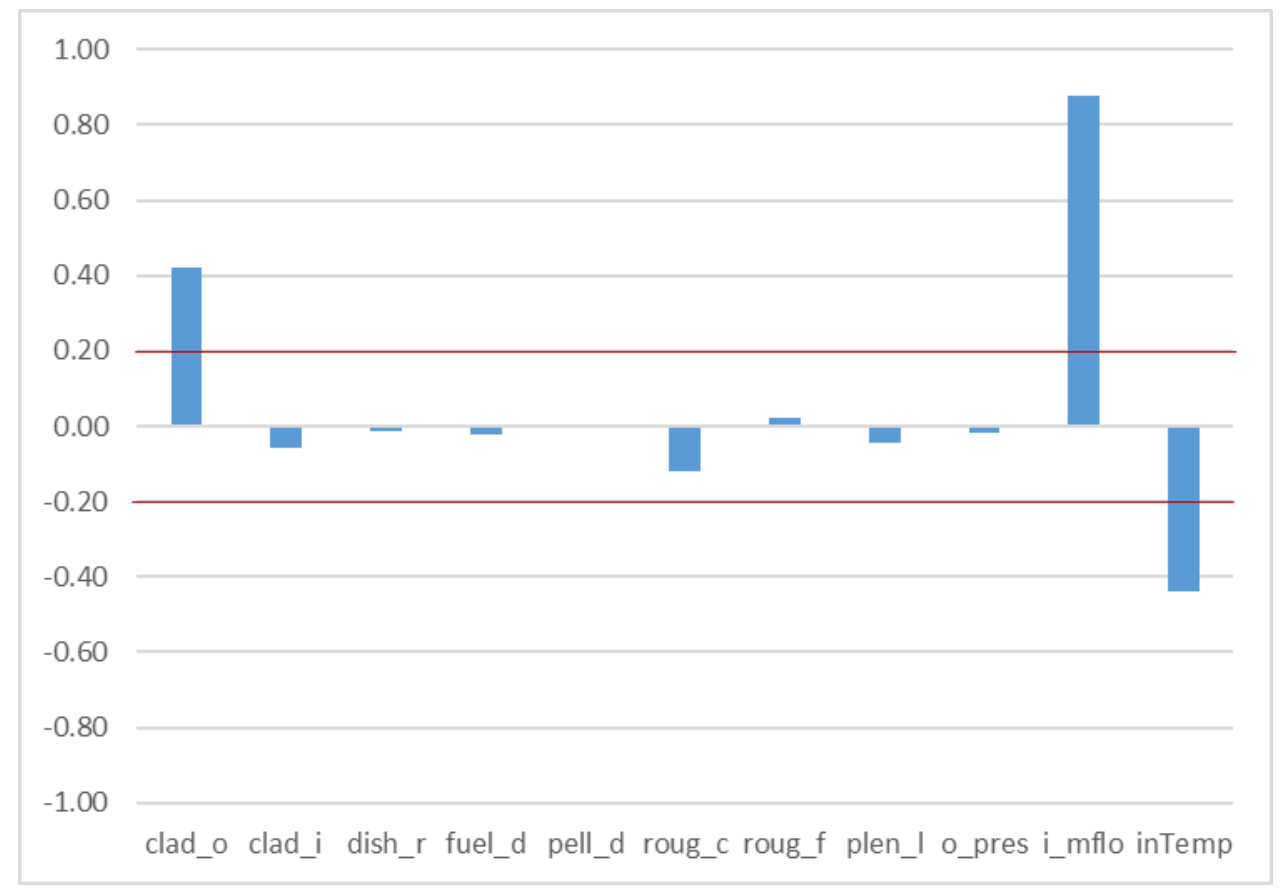

Figure 5.20 Sensitivity Analysis of the transient case.

Table 5.8 supports the results of Figure 5.20.

Table 5.8 Sensitivity Analysis of the transient case.

\begin{tabular}{|l|l|l|l|}
\hline Variable & units & variable name & PRCC \\
\hline Cladding Outer diameter & $\mathrm{m}$ & clad_o & 0.4209 \\
\hline Cladding Inner Diameter & $\mathrm{m}$ & clad_i & -0.0567 \\
\hline
\end{tabular}


Development and validation of a multi-scale and multi-physics methodology for the safety analysis of fast transients in Light Water Reactors

\begin{tabular}{|l|l|l|l|}
\hline Pellet Dish Radius & $\mathrm{m}$ & dish_r & -0.0137 \\
\hline Fuel Density & $\%$ & fuel_d & -0.0223 \\
\hline Pellet Diameter & $\mathrm{m}$ & pell_d & -0.0060 \\
\hline Cladding Roughness & $\mu \mathrm{m}$ & roug_c & -0.1194 \\
\hline Pellet Roughness & $\mu \mathrm{m}$ & roug_f & 0.0217 \\
\hline Plenum Length & $\mathrm{m}$ & plen_l & -0.0424 \\
\hline Outlet Pressure & $\mathrm{bar}$ & o_pres & -0.0186 \\
\hline Inlet Mass Flow & $\mathrm{kg} / \mathrm{s}$ & i_mflo & 0.8780 \\
\hline Inlet Temperature & $\mathrm{K}$ & inTemp & -0.4400 \\
\hline
\end{tabular}

In view of the results in Table 5.8 and Figure 5.20, it can be concluded that the input parameters affecting significantly the uncertainty of the Minimum CPR correspond to the operation conditions of mass flow and inlet temperature of the coolant. The Minimum CPR parameter is directly dependent on the Critical Heat Flux (CHF) correlation, and this parameter is determined by coolant conditions such as the flow quality, therefore it is expectable to highlight the uncertainty in the coolant inlet conditions as main source of uncertainty in the Minimum CPR. Furthermore, the Minimum CPR, as well as the $\mathrm{CHF}$, depend in the heat transfer capacity of the fuel rod. For this variable, parameters such as the cladding diameter and the roughness play a relevant role, and therefore, the uncertainty of these parameters affect to the uncertainty of the prediction of the Minimum CPR in the methodology.

\subsection{Conclusions on the application case.}

The proposed simulation methodology has been applied to an AOO, namely TT. This event corresponds to the TT event of KKL of fuel cycle 18, therefore the results of the application of the methodology are compared against real plant data. According to the USNRC, the safety variable to be analyzed is the Minimum CPR and the pressure of the reactor vessel. Safety criterion for the limit of the pressure achieved during the TT is defined with the system model, where the reactor vessel is modelled. Conversely, the Minimum CPR is a more local phenomenon, which can be predicted in a Best Estimate way, if the appropriate scale and the known physics are applied. For that purpose, the simulation of the TT event has been iterated from a coarse mesh in a system model to the simulation of the critical fuel pin. Moreover, the TH, the NK and the thermo-mechanics have been applied. 
The presented methodology shows the results of increasing step-by-step the detail level, from a coarse mesh to the pin level. The results of the calculated Minimum CPR change depending on the scale and the physics simulated of the corresponding model. These differences between the prediction are due to the fact that a detailed scale provides a better approximation of such a local phenomenon. Moreover, the different steps of the methodology added different physics that allowed accounting more parameters for the prediction of the Minimum CPR.

In addition, in order to complement the Best Estimate results, an Uncertainty and Sensitivity Analysis has been performed. The scientific literature provided the relevant input parameters affecting the prediction of the CPR. By means of the Wilks method the methodology generates a sample of 146 cases according to the uncertainty of the proposed input parameters and afterwards the Confidence Interval is generated. The 95/95 criterion was meet since more than the $95 \%$ of the sample cases fall in a Confidence Interval of the $95 \%$. Therefore, the Safety Criterion of the Minimum CPR is met.

The proposed methodology has proven to be a useful tool for the evaluation of the corresponding safety variables according to the regulation of the USNRC. Different models where defined for the application of the methodology to the TT event showing good agreement and an enhancement of the prediction of the Minimum CPR due to the BE approach.

Future work can be headed to provide a channel-by-channel core model for the vessel of TRACE. This step would allow skipping the CTF-UPVIS/PARCS simulation. On the other hand, the CTF-UPVIS/PARCS model could be enhanced tracking first the critical fuel channel, and then replacing the critical fuel channel, which is made of a lumped fuel channel model, by a detailed pin-by-pin model. This procedure would skip the simulation of the thermal-hydraulic subchannel model, accounting in addition the effect of the heat transfers between the adjacent subchannels. 


\section{Chapter 6 \\ Conclusions and Future Work}

The present document, in its first chapter, proposed the objective of developing, verifying and validating a multi-scale and multi-physics methodology for the analysis of fast transients in LWRs using the state-of-the-art codes. The development of this methodology had as main reference the current standards and recommendations of the IAEA and used the guidelines of the USNRC and ENSI. The purpose was adding value to the different codes that are used in different fields of the Nuclear Engineering to produce energy by means of combining their capabilities toward a single aim. This aim was analyzing the target safety variables of a postulated transient scenario with the corresponding physics at the needed scale. The results have been a Best Estimate approach for predicting the core behavior, which yields more realistic results that allow a better adjustment of the safety margins resulting hence in a more cost-efficient operation and design of the NPP and a safer assessment of the core conditions.

This objective has been achieved after the consolidation of the necessary background not only in Nuclear Safety Analysis but also in the corresponding state-of-the-art tools and the current status of the physical knowledge regarding the behavior of LWRs. 


\subsection{Conclusions.}

On the one hand, the methodology is adjusted to the guidelines and regulations of different national and international organizations that focus their work in evaluating which aspects of the operation of a NPP are relevant and have to be bounded in order to assure a safe functioning without any damage to the environment, public and staff. Therefore, the proposed methodology has been developed keeping in mind that the use of the different codes and their combined use must be focused in retrieving the necessary safety variables and defining the standard scenarios to be analyzed. This analysis helped to define the level of accuracy that the methodology had to set for its calculations, so the results can be introduced in a standard framework that can be acknowledge for the different Nuclear Authorities.

On the other hand, the current state-of-the-art simulation tools were analyzed in detail in order to define a route plan that step-by-step incorporates the needed simulation tools that compose the framework of the so called Best Estimate approach. The different physics used for analyzing the core behavior of a LWR have been analyzed in detail and the result has been a selection of different code families that retrieve the necessary predictions using the physics known at the moment and also the feedback mechanisms between such physics. In addition, different phenomena relevant for the safety analysis was analyzed in detail, so it can be defined the necessary scale of analysis. This work was headed to allow the user to perform a detailed evaluation of safety variables, if necessary. As commented in previous sections, certain phenomena of the reactor core, such as the dryout of a fuel rod, has a very local behavior. The proposed methodology offers a set of physics and scales to perform a BE approach, instead of analyzing such scenario in a coarser scale or assuming conservative models and conditions.

As a first task of the application of the methodology, the author of this thesis has gathered and analyzed the corresponding Regulatory Guidelines to perform the corresponding Safety Assessment. Each of the applied steps of this methodology is the result of achieving the partial objectives of this work. First of all, the first step has generated the 3D cross-section libraries for a proper analysis of the NK and its role of the TT transient. To be more specific, the cross-section libraries have been generated using the SIMTAB methodology. Secondly, the generated cross-section libraries where used in a TH/NK coupled model using the coupled codes TRACE/PARCS. This step simulated the general behavior of the reactor core, including different plant systems as the reactor turbine system, the recirculation system and the reactor pressure vessel. This step provided a first approach of the behavior of the code used in the following step, i.e. the coupled TH/NK 3D subchannel core analysis. In this third step, the objective was to increase the detail level of a BE simulation of the core. The results of this step allowed to track the critical fuel channel according to the MCPR criterion. In this step, the core was modelled channel-by-channel using the coupled code CTF-UPVIS/PARCS. The analysis of this last step is improved and complemented adding the evaluation of the fuel behavior with a pin level. The partial objective is to add a BE approach of the thermodynamics of the 
Development and validation of a multi-scale and multi-physics methodology for the safety analysis of fast transients in Light Water Reactors

fuel cladding at pin level. This objective was fulfilled using the suite FRAPCON/FRAPTRAN. Finally, the results are complemented applying the Uncertainty and Sensitivity Analysis. This step has been designed as a reliable way to provide the margins of the predicted safety variable, i.e. the MCPR. This part of the methodology was complemented using the DAKOTA toolkit. Moreover, as part of the development of the methodology, the management of the data flow has been handled using MATLAB-, FORTRAN and LINUX-scripts. The purpose of this partial objective has been avoiding an excessive interference of the user to pre- and post-process the input and output information.

Finally, the analysis of the results of the simulations undertaken by the different codes and the different scales revealed the necessary schedule for pre- and post-processing the data. This task was necessary to assure a correct flow of information, since the results yielded by one step of the methodology are used to feed the input of the next step. In addition, the influence of the user has been avoided by means of automatizing the data flow process. This has been made with interface applications programmed in different conventional languages and tools so the user only needs to set the corresponding safety criterion, and the applications pre- and post-processing the data will track, retrieve and load the simulation conditions according to the critical component based on the selected safety limit.

The result is a methodology headed to the prediction to the safety variables according the Nuclear Safety Standards designed to simulate the corresponding scenarios, becoming a useful tool not only for delivering the necessary periodical results to the corresponding Nuclear Authority, but working as a flexible tool for the support in the daily safety analysis needed in a NPP.

The design of the proposed methodology has been verified and validated in order to assess its capacity as simulation tool. Each step of the methodology has been tested including comparisons with reference codes of the Nuclear Industry and comparison of the results against plant data. The chosen scenario has been the Turbine Trip of KKL fuel cycle 18 . The necessary guidelines were reviewed in order to set the target variables in such scenario. The reference has been the Guidelines of the United States Nuclear Regulatory Commission. According to these regulations, the target variable has been the minimum Critical Power Ratio. Regarding this scenario, it was concluded that a detailed scale is needed for the prediction of such local phenomena, but also the coupling between the Thermal-Hydraulics of the core and the Neutron Kinetics was necessary for an accurate evaluation of the evolution of the core behavior in this fast transient.

The methodology has allowed to evaluate the whole system behavior, and step by step, get deep in the scale until analyzing the thermal-hydraulics and fuel performance at pin level. As it has been proved, using this multi-scale and multi-physics feature has yielded realistic results, that otherwise would have been categorized as conservative, forcing the operation of the NPP to assume wider safety margins. The validation of the results showed good agreement in the core behavior as the prediction of core power and pressure 
vessel shows. The general behavior of the core accounting the thermal-hydraulic and neutron kinetic feedback was used to introduce the boundary conditions of the core in a channel-by-channel model, allowing the location of the critical fuel channel according to the Minimum CPR criteria. The proposed methodology has been defined in order to use a database of pin-by-pin fuel channel models for a subchannel thermal-hydraulic code, in this case CTF-UPVIS. The use of this code at this scale provides Best Estimate approach used for locating the critical fuel pin. Finally, these results were loaded in a fuel pin model for the fuel performance analysis with FRAPTRAN/FRAPCON suite. The application of the different steps reveals different approaches of the evolution of the Minimum CPR, defining in its last step the more realistic approach. This result has been complemented with the Uncertainty and Sensitivity analysis that quantifies the uncertainty of the prediction by defining a Confidence Interval of $95 \%$. The method applied using DAKOTA toolkit showed that the result meets the 95/95 criterion, which meets as well the requirements of the Regulatory Guidelines. Moreover, the features of the methodology allowed to perform a Sensitivity Analysis that was used to evaluate which input variables affect most to the uncertainty in the prediction of the Minimum CPR. The results of this application case have been published in a Journal Paper of the Nuclear Engineering and Design into different parts, revealing the impact of the work developed within this $\mathrm{PhD}$ work.

\subsection{Future work.}

The development of the proposed methodology achieved a solid milestone as their results prove. Nonetheless, different aspects can be developed in future work in order to enhance the results provided by this methodology.

First of all, it must be accounted that the conventional application of this methodology for the Deterministic Safety Analysis of a NPP will require the continuous updating of models, cross section data and also code versions. For instance, the database of pin-bypin fuel models in KKL will be expanded including fuel models for other codes and also the possible new models that can be included in the future. Besides, the continuous functioning of the power plant yields the necessity of updating the cross-section libraries after each fuel cycle.

Secondly, the user of this methodology must be aware the research in the field of Deterministic Safety Analysis and Nuclear Simulation Technology keeps evolving and advancing every day. The state-of-the-art regarding physical models is being updated with new experiments and benchmarks that improve correlations and more accurate physical models. These improvements in the field of Nuclear Engineering must be applied to the corresponding simulation tools used in the methodology. The purpose of this is to maintain the Best Estimate approach of this work.

Moreover, the development of computer sciences will provide better simulation tools that will allow different mathematical schemes for the prediction of the safety analysis. 
Development and validation of a multi-scale and multi-physics methodology for the safety analysis of fast transients in Light Water Reactors

Therefore, the improvement and updating of a multi-physics and multi-scale methodology will always something to keep in mind.

In addition, the satisfactory preliminary results presented in the application case of Chapter 5.1, encourage to continue with further validation cases. The good results and team work between UPV and KKL pave the way to future collaborations that may allow the application of this methodology to other Plant Transients. The availability of plant data is an aspect that facilitates a solid validation of the different features of this methodology. The evaluation of other transient cases, such as the stability transients, can be a very interesting application case that will allow exploring other capabilities of the proposed methodology and the research area of BE simulations and Uncertainty Analysis.

On the other hand, there is an additional interest in the further development of Uncertainty Quantification that could be applied to this work in the future. The analysis of the uncertainty propagation is another interesting that is considered very suitable for this methodology. The proposed methodology has proven the capability of exchange data from step to step. With the corresponding data, it can be possible to model the sampling and analysis process from the first step to the final result, evaluating through the different steps the propagation of the uncertainty.

Lastly, it could be of significant interest to be able to validate the methodology to plant transients of PWRs. Unfortunately, as commented, plant data is not always available, and for this future objective, it will be necessary to wait for further projects.

To summarize, besides the commented aspects, there are short-term lines of future work to be developed.

- Starting on the step of generating Cross Section Libraries, the GenPMAXS methodology using CASMO data is still in development phase. Further application cases will be carried out to provide the methodology with solid PMAXS files retrieved from CASMO. This step of the methodology will be validated and verified in order to make the GenPMAXS interface a reference part of the methodology for the generation of Cross Section Libraries.

In addition, it must be accounted that CASMO/SIMULATE is a commercial tool very extended in the Nuclear Engineering field due to its proven capabilities. Nevertheless, other recent sources for generating Cross Section Libraries can be investigated. Currently, the code POLARIS can be used with the GenPMAXS interface, and its transport methods can be evaluated for generating the PMAXS libraries in future work.

- The analysis of the system scale uses a 3D core model where the fuel channels are lumped in 3 average channels. A more accurate definition of this model will account a channel-by-channel core model. This will mean more calculation power to be used, but the results will be enhanced since the thermal-hydraulic 
behavior of each channel will be accounted, instead using averaged values for each lumped channel.

The purpose of this future work is to provide more detailed core conditions to the subchannel thermal-hydraulic and neutron kinetics analysis step. This step was undertaken using a CTF-UPVIS channel-by-channel model, coupled with PARCS. The purpose is to locate the critical fuel channel. However, performing the previous step with a channel-by-channel core model in the TRACE input deck will already yield the critical fuel channel. Therefore, afterwards this fuel channel will be defined as a pin-by-pin fuel channel, in the channel-by-channel core model of CTF-UPVIS input deck. The improvement of this model will account more realistic boundary conditions in this step for the heat transfer of the pin-by-pin model through the adjacent fuel channels. Notice that this improvement will help to skip the next step, the pin-by-pin fuel channel analysis, allowing to go directly to the fuel performance analysis at pin level.

- The last step of the proposed methodology quantifies the uncertainty in the boundary conditions and different correlations for the prediction of the Minimum CPR. The analysis was undertaken to evaluate how these uncertainties are propagated through the simulation tool. This step needed the corresponding analysis of the scientific literature that helped to define the probability density functions of the input variables, and the definition of it was also supported in the engineering criteria of the author of this work. Nevertheless, it will add a better approach of the propagation of the uncertainties the analysis from the first step, and how these uncertainties are propagated from one step to another.

As observed, there are different future lines to be tackled in short and long terms. Furthermore, the development of this methodology will have to be adapted to the corresponding LWR and the available state-of-the-art tools that will integrate this multi-scale and multi-physics methodology. Nonetheless, author counts on the fact that the work developed and presented here will be a useful proof and support of the improvement of the Deterministic Safety Analysis towards a more cost-efficient and safer way to design the operation and analyzing the behavior of Nuclear Power Plants. 


\section{Chapter 7 \\ Contributions}

The work developed in this thesis has yield a series of contributions regarding journal papers, and different international and national congress papers. Moreover, the project of the thesis has been followed through different reports in relationship to the different $\mathrm{R} \& \mathrm{D}$ projects.

\subsection{Publications in indexed journals.}

- Title: A multi-scale and multi-physics simulation methodology with the stateof-the-art tools for safety (PART I)

Authors: Patricio Hidalga, Agustín Abarca, Rafael Miró, Abdelkrim Sekrhi, Gumersindo Verdú

Journal: Nuclear Engineering and Design (ISSN 0029-5493)

Volume: 350 Pages: 205-213

Year: 2019

DOI: 0.1016/j.nucengdes.2019.05.009 
- Title: A multi-scale and multi-physics simulation methodology with the stateof-the-art tools for safety (PART II)

Authors: Patricio Hidalga, Agustín Abarca, Rafael Miró, Abdelkrim Sekrhi, Gumersindo Verdú

Journal: Nuclear Engineering and Design (ISSN 0029-5493)

Volume: 350 Pages: 195-204 Year: 2019

DOI: 0.1016/j.nucengdes.2019.05.008

\subsection{Oral participation in International Conferences.}

- $\quad$ Title: Multi-Scale and multi-physics methodology for LWR safety analysis plus uncertainty

Authors: P. Hidalga, R. Miró, A. Sekhri.

Congress: 12th International Topical Meeting on Nuclear Thermal-Hydraulics, Operation and Safety (NUTHOS-12)

Location: Qingdao, China

Date: $14-18 / 10 / 2018$

- $\quad$ Title: BEPU methodology for the void fraction prediction in PWR fuel bundles validated with the PSBT benchmark for steady-state and transient cases.

Authors: P. Hidalga, R. Miró, A. Sekhri.

Congress: The Fourth International Conference on Physics and Technology of Reactors and Applications (PHYTRA4).

Location: Marrakech, Morocco_ Date:19/09/2018

- Title: Evaluation of a BEPU methodology with state-of-the-art tools in a nonrealistic Turbine Trip with ATWS for a BWR

Authors: P. Hidalga, A. Abarca, R. Miró, A. Sekhri.

Congress: Best Estimate Plus Uncertainty International Conference (BEPU 2018)

Location: Lucca, Italy

Date: $13-18 / 05 / 2018$

- $\quad$ Title: Uncertainty and sensitivity analysis in void fraction prediction with CTFUPVIS of the PSBT and BFBT Fuel Models for transient cases.

Authors: P. Hidalga, A. Abarca, R. Miró. 
Development and validation of a multi-scale and multi-physics methodology for the safety analysis of fast transients in Light Water Reactors

Congress: Best Estimate Plus Uncertainty International Conference (BEPU 2018)

Location: Lucca, Italy

Date: $13-18 / 05 / 2018$

- Title: CTF working activities at Universitat Politècnica de València

Authors: P. Hidalga, A. Abarca, R. Miró, G. Verdú.

Congress: Fourth CTF User's Group Meeting (CTF-4)

Location: Erlangen, Germany

Date: 08/05/2017

- $\quad$ Title: Integrated Methodology for the analysis of fuel integrity in high burnup fuel assemblies using CTF-UPVIS, FRAPCON and FRAPTRAN codes.

Authors: P. Hidalga, A. Abarca, R. Miró, G. Verdú.

Congress: Nuclear Reactor Thermal Hydraulics International Topical meeting 17th (NURETH-17)

Location: Xian, China

Date: 3-8/09/2017

- Title: Implementation of Groeneveld Critical Heat Flux look-up table in FRAPTRAN and assessment against the post-dryout experiments performed at the Royal Institute of Technology.

Authors: P. Hidalga, A. Abarca, R. Miró, G. Verdú.

Congress: Nuclear Reactor Thermal Hydraulics International Topical meeting 17th (NURETH-17)

Location: Xian, China

Date: 3-8/09/2017

- $\quad$ Title: Thermal-Hydraulic uncertainty and sensitivity analysis applied to BWR fuel assemblies using CTF-UPVIS and DAKOTA

Authors: P. Hidalga, A. Abarca, R. Miró, G. Verdú.

Congress: Nuclear Reactor Thermal Hydraulics International Topical meeting 17th (NURETH-17)

Location: Xian, China

Date: 3-8/09/2017

- Title: Verification of CTF/PARCSv3.2 coupled code in a turbine trip scenario 
Authors: A. Abarca, P. Hidalga, R. Miró, G. Verdú, A. Sekhri.

Congress: 2017 International Congress on Advances in Nuclear Power Plants (ICAPP2017)

Location: Fukui and Kyoto, Japan

Date: $24 / 04 / 2017$

ISBN: 978-4-89047-167-6

- Title: Validation of the subchannel code CTF against the benchmark data of the OECD/NEA PSBT

Authors: A. Abarca, P. Hidalga, R. Miró, T. Barrachina, G. Verdú.

Congress: ANS Reactor Physics Topical Meeting (PHYSOR 2014)

Location: Kyoto, Japan

Date: $3 / 10 / 2014$

ISBN: 978-0-89448-095-9

- $\quad$ Title: TRACE coupled with PARCS benchmark against Leibstadt plant data during the Turbine Trip Test

Authors: P. Hidalga, A. Sekhri, P. Bauman, D. Morera, R. Miró, T. Barrachina, G. Verdú.

Congress: International Nuclear Atlantic Conference (INAC 2013)

Location: Recife, Brazil

Date: $24-29 / 11 / 2013$

ISBN: 978-85-99141-05-2

- Title: Validation of the subchannel code CTF against the benchmark data of the OECD/NEA PSBT.

Authors: Agustín Abarca; Rafael Miró; Hidalga-García-Bermejo Patricio; Teresa Barrachina; Gumersindo Verdú

Congress: ANS Reactor Physics Topical Meeting (PHYSOR 2014). The Role of Reactor Physics towards a Sustainable Future

Location: Kyoto, Japan

Date: $3 / 10 / 2014$

ISBN: 978-0-89448-095-9

- Title: CTF working activities at Universitat Politècnica de València

Authors: P. Hidalga, A. Abarca; R. Miró; G. Verdú; 
Development and validation of a multi-scale and multi-physics methodology for the safety analysis of fast transients in Light Water Reactors

Congress: Fourth CTF User's Group Meeting (CTF-4)

Location: Erlangen, Deutschland Date: 8/05/2017

\subsection{Oral presentations in national conferences.}

- $\quad$ Title: Análisis de sensibilidad e incertidumbre en la predicción de fracción de huecos del código CTF-UPVIS validado con los experimentos BFBT y PSBT de la OECD/NEA.

Authors: P. Hidalga, R. Miró.

Congress: 43 Reunión Anual de la Sociedad Nuclear Española

Location: Ávila, España

Data: $26-28 / 09 / 2018$

- Title: Metodología para el análisis de la integridad de combustibles de alto quemado por medio de los códigos CTF y FRAPTRAN.

Authors: P. Hidalga, A. Abarca, R. Miró, G. Verdú.

Congress: 43 Reunión Anual de la Sociedad Nuclear Española

Location: Málaga, España

Data: $4 / 10 / 2017$

- $\quad$ Title: Análisis de sensibilidad e incertidumbre termohidráulico a escala subcanal en elementos combustibles de reactores tipo LWR con los códigos CTF y DAKOTA.

Authors: P. Hidalga, A. Abarca, R. Miró, G. Verdú.

Congress: 43 Reunión Anual de la Sociedad Nuclear Española

Location: Málaga, España

Data: $4 / 10 / 2017$

- $\quad$ Title: Validación de librerías PMAXS de secciones eficaces por medio del código PARCS 3.2 en casos estacionarios de la C.N. Cofrentes (España) y KKL (Suiza).

Authors: P. Hidalga, A. Sekhri, P. Baumann, R. Miró, G. Verdú.

Congress: 41 Reunión Anual de la Sociedad Nuclear Española

Location: La Coruña, España

Data: $23 / 09 / 2015$

ISBN: 978-84-608-2453-4 
- $\quad$ Title: Mejora del modelo acoplado TRACE/PARCS de la planta KKL empleando la simulación del evento disparo de turbina de 2001.

Authors: P. Hidalga, A. Sekhri, P. Papadopoulos, R. Miró, T. Barrachina, G. Verdú.

Congress: 41 Reunión Anual de la Sociedad Nuclear Española

Location: La Coruña, España

Data: 23/09/2015

ISBN: 978-84-608-2453-4

- $\quad$ Title: Validación del modelo acoplado TRACE/PARCS de KKL por medio de los datos de planta del evento disparo de turbina de 2001.

Authors: P. Hidalga, A. Sekhri, P. Baumann, D. Morera, R. Miró, T. Barrachina, G. Verdú.

Congress: 40 Reunión Anual de la Sociedad Nuclear Española

Location: Valencia, España

Data: 01/10/2014

ISBN: 978-84-697-1656-4

- $\quad$ Title: Validación del código de subcanal CTF por medio de los resultados experimentales del benchmark PSBT de la OECD/NEA.

Authors: Hidalga-García-Bermejo, Patricio; Miró Herrero, Rafael; Barrachina Celda, Teresa María; Abarca Giménez, Agustín; Verdú Martín, Gumersindo Jesús.

Congress: 40 Reunión Anual de la Sociedad Nuclear Española

Location: Valencia, España

Data: 01/10/2014

ISBN: 978-84-697-1656-4

\subsection{Development of projects in the framework of collaboration with dif- ferent private companies that generated a series of technical reports.}

- Title: THERMAL-HYDRAULIC/NEUTRONIC CODE TRACE/PARCS

Entity responsible of the development: Universitat Politècnica de València.

Supervisor: Prof. Dr. Rafael Miró Herrero

Authors/Research staff: Patricio Hidalga García-Bermejo 
Development and validation of a multi-scale and multi-physics methodology for the safety analysis of fast transients in Light Water Reactors

Financing Entity: Kernkraftwerk Leibstadt, AG.

Initiation date: $28 / 12 / 2011$

Duration: 28/01/2013

- $\quad$ Title: METHODOLOGY FOR COUPLED FAST TRANSIENTS IN BWR DETERMINISTIC SAFETY ANALYSIS WITH STATE-OF-THE-ART TOOLS

Entity responsible of the development: Universitat Politècnica de València.

Supervisor: Prof. Dr. Rafael Miró Herrero

Authors/Research staff: Patricio Hidalga García-Bermejo

Financing Entity: Kernkraftwerk Leibstadt, AG.

Initiation date: $11 / 03 / 2013$

Duration: 11/01/2014

- $\quad$ Title: METHODOLOGY FOR COUPLED FAST TRANSIENTS IN BWR DETERMINISTIC SAFETY ANALYSIS WITH STATE-OF-THE-ART TOOLS

Entity responsible of the development: Universitat Politècnica de València.

Supervisor: Prof. Dr. Rafael Miró Herrero

Authors/Research staff: Patricio Hidalga García-Bermejo

Financing Entity: Kernkraftwerk Leibstadt, AG.

Initiation date: $28 / 07 / 2015$

Duration: 28/01/2017

- Title: BELDO PROJECT ANALYSIS FOR DRY-OUT, CRITICAL FILM THICKNESS AND DENSITY WAVE OSCILLATION

Entity responsible of the development: Universitat Politècnica de València.

Supervisor: Prof. Dr. Rafael Miró Herrero

Authors/Research staff: Patricio Hidalga García-Bermejo, Dr. Agustín Abarca

Financing Entity: Kernkraftwerk Leibstadt, AG.

Initiation date: 03/12/2019 Duration: 03/04/2020

- Title: TRACE/PARCS PROJECT MULTIPHYSICS MULTISCALE METHODOLOGY

Entity responsible of the development: Universitat Politècnica de València.

Supervisor: Prof. Dr. Rafael Miró Herrero

Authors/Research staff: Patricio Hidalga García-Bermejo

Financing Entity: Kernkraftwerk Leibstadt, AG.

190 


\subsection{Technical reports produced in the framework of the Thesis Project}

\section{- $\quad$ Title: Isirym/Senubio-THN-0015-12.01. HELIOS KKL INPUT EDITING FOR GENPMAX SUMMARY}

Entity responsible of the development: Universitat Politècnica de València.

Supervisor: Prof. Dr. Rafael Miró Herrero

Authors/Research staff: Patricio Hidalga García-Bermejo, Prof. Dr. Rafael Miró Herrero, Prof. Dr. Gumersindo Verdú Martín

Financing Entity: Kernkraftwerk Leibstadt, AG.

Submittal Date: 03/09/2013

- Title: Isirym/Senubio-THN-0026-14.01 Executive Summary: Report of differences between KKL and ISIRYM versions of SIMULATE while core follow execution Entity responsible of the development: Universitat Politècnica de València.

Supervisor: Prof. Dr. Rafael Miró Herrero

Authors/Research staff: Patricio Hidalga García-Bermejo, Prof. Dr. Rafael Miró Herrero Financing Entity: Kernkraftwerk Leibstadt, AG.

Submittal Date: 15/06/2014

- Title: Isirym/Senubio-THN-0026-14.02 Validation of SIMTAB methodology for Cross Section generation for PARCS against SIMULATE data

Entity responsible of the development: Universitat Politècnica de València.

Supervisor: Prof. Dr. Rafael Miró Herrero

Authors/Research staff: Patricio Hidalga García-Bermejo, Prof. Dr. Rafael Miró Herrero

Financing Entity: Kernkraftwerk Leibstadt, AG.

Submittal Date: 16/06/2014

- $\quad$ Title: Isirym/Senubio-THN-0026-14.03 Report of analysis of PARCS performance in steady state simulations with PMAXS/CASMO4 Cross Section Libraries 
Development and validation of a multi-scale and multi-physics methodology for the safety analysis of fast transients in Light Water Reactors

Entity responsible of the development: Universitat Politècnica de València.

Supervisor: Prof. Dr. Rafael Miró Herrero

Authors/Research staff: Patricio Hidalga García-Bermejo, Prof. Dr. Rafael Miró Herrero

Financing Entity: Kernkraftwerk Leibstadt, AG.

Submittal Date: 15/10/2014

- Title: Senubio/Isirym-THN-0015-15.01 Preliminary results of PMAXS libraries obtained with CASMO version 5 from KKL

Entity responsible of the development: Universitat Politècnica de València.

Supervisor: Prof. Dr. Rafael Miró Herrero

Authors/Research staff: Patricio Hidalga García-Bermejo

Financing Entity: Kernkraftwerk Leibstadt, AG.

Submittal Date: $15 / 05 / 2015$

- Title: Senubio/Isirym-THN-0005-10.01 Executive Summary: Methodology for generating Cross Section libraries

Entity responsible of the development: Universitat Politècnica de València.

Supervisor: Prof. Dr. Rafael Miró Herrero

Authors/Research staff: Patricio Hidalga García-Bermejo, Prof. Dr. Rafael Miró Herrero

Financing Entity: Kernkraftwerk Leibstadt, AG.

Submittal Date: 18/12/2015

- Title: Senubio/Isirym-THN-0005-09.01 Project Tasks: Executive Summary

Entity responsible of the development: Universitat Politècnica de València.

Supervisor: Prof. Dr. Rafael Miró Herrero

Authors/Research staff: Patricio Hidalga García-Bermejo, Prof. Dr. Rafael Miró Herrero, Prof. Dr. Gumersindo Verdú Martín

Financing Entity: Kernkraftwerk Leibstadt, AG.

Submittal Date: 23/05/2016 
- Title: Senubio/Isirym-THN-0026-16.03 FRAPCON/FRAPTRAN fuel pin models of the fuel bundle SVEA-96 Optima2 for the fuel cycle 30

Entity responsible of the development: Universitat Politècnica de València.

Supervisor: Prof. Dr. Rafael Miró Herrero

Authors/Research staff: Patricio Hidalga García-Bermejo, Prof. Dr. Rafael Miró Herrero, Dr. Agustín Abarca Jiménez

Financing Entity: Kernkraftwerk Leibstadt, AG.

Submittal Date: 01/12/2016

- Title: Senubio/Isirym-THN-0026-16.04 Uncertainty and Sensitivity analysis applied to the BELDO project in KKL

Entity responsible of the development: Universitat Politècnica de València.

Supervisor: Prof. Dr. Rafael Miró Herrero

Authors/Research staff: Patricio Hidalga García-Bermejo, Prof. Dr. Rafael Miró Herrero, Dr. Agustín Abarca Jiménez

Financing Entity: Kernkraftwerk Leibstadt, AG.

Submittal Date: 01/12/2016

- Title: Isirym/Senubio-THN-0031-17.02 Preliminary results for Uncertainty and Sensitivity analysis applied to the BELDO project in KKL

Entity responsible of the development: Universitat Politècnica de València.

Supervisor: Prof. Dr. Rafael Miró Herrero

Authors/Research staff: Patricio Hidalga García-Bermejo, Dr. Agustín Abarca Jiménez

Financing Entity: Kernkraftwerk Leibstadt, AG.

Submittal Date: 27/05/2017

- Title: Isirym/Senubio-THN-0032-17.03 BELDO 17: Justification and references for the CTF model SVEA96O2 design

Entity responsible of the development: Universitat Politècnica de València.

Supervisor: Prof. Dr. Rafael Miró Herrero

Authors/Research staff: Patricio Hidalga García-Bermejo 
Development and validation of a multi-scale and multi-physics methodology for the safety analysis of fast transients in Light Water Reactors

Financing Entity: Kernkraftwerk Leibstadt, AG.

Submittal Date: 03/10/2017

- $\quad$ Title: Isirym/Senubio-THN-0032-17.01 BELDO 17: PROJECT FOLLOW UP

Entity responsible of the development: Universitat Politècnica de València.

Supervisor: Prof. Dr. Rafael Miró Herrero

Authors/Research staff: Patricio Hidalga García-Bermejo, Dr. Agustín Abarca

Financing Entity: Kernkraftwerk Leibstadt, AG.

Submittal Date: 21/12/2017

- $\quad$ Title: Isirym/Senubio-THN-0031-17.04a METHODOLOGY FOR COUPLED FAST TRANSIENTS IN BWR DETERMINISTIC SAFETY ANALYSIS WITH STATE-OF-THE-ART

Entity responsible of the development: Universitat Politècnica de València.

Supervisor: Prof. Dr. Rafael Miró Herrero

Authors/Research staff: Patricio Hidalga García-Bermejo, Dr. Agustín Abarca

Financing Entity: Kernkraftwerk Leibstadt, AG.

Submittal Date: 30/12/2017

- Title: Isirym/Senubio-THN-0031-17.04b Report of the modelling and design of the CTF-UPVIS input deck for SVEA 96 Optima 2 fuel assembly

Entity responsible of the development: Universitat Politècnica de València.

Supervisor: Prof. Dr. Rafael Miró Herrero

Authors/Research staff: Patricio Hidalga García-Bermejo, Dr. Agustín Abarca

Financing Entity: Kernkraftwerk Leibstadt, AG.

Submittal Date: 30/12/2017

- Title: Isirym/Senubio-THN-0031-17.05 Follow up of the project task regarding Cross Section Libraries Generation

Entity responsible of the development: Universitat Politècnica de València. 
Supervisor: Prof. Dr. Rafael Miró Herrero

Authors/Research staff: Patricio Hidalga García-Bermejo

Financing Entity: Kernkraftwerk Leibstadt, AG.

Submittal Date: 30/12/2017

- Title: Isirym/Senubio-THN-0033-18.01 METHODOLOGY FOR COUPLED

3D TRANSIENTS IN BWR DETERMINISTIC SAFETY ANALYSIS FOR LICENSING PURPOSES

Entity responsible of the development: Universitat Politècnica de València.

Supervisor: Prof. Dr. Rafael Miró Herrero

Authors/Research staff: Patricio Hidalga García-Bermejo

Financing Entity: Kernkraftwerk Leibstadt, AG.

Submittal Date: 17/04/2018

- Title: Isirym/Senubio-THN-0032-18.02 BELDO 17: PROJECT FOLLOW UP: TASK 2

Entity responsible of the development: Universitat Politècnica de València.

Supervisor: Prof. Dr. Rafael Miró Herrero

Authors/Research staff: Patricio Hidalga García-Bermejo, Dr. Agustín Abarca

Financing Entity: Kernkraftwerk Leibstadt, AG.

Submittal Date: 30/06/2018

- $\quad$ Title: Isirym/Senubio-THN-0033-18.03 SUMMARY OF RESULTS OF THE GENERATION PROCEDURE OF CROSS SECTION LIBRARIES FOR THE DIFFUSION CODE PARCS

Entity responsible of the development: Universitat Politècnica de València.

Supervisor: Prof. Dr. Rafael Miró Herrero

Authors/Research staff: Patricio Hidalga García-Bermejo

Financing Entity: Kernkraftwerk Leibstadt, AG.

Submittal Date: 11/07/2018 
Development and validation of a multi-scale and multi-physics methodology for the safety analysis of fast transients in Light Water Reactors

- Title: Isirym/Senubio-THN-0032-18.04 Evaluation of the capabilities of code CTF-UPVIS for the simulation and analysis of the phenomena regarding BELDO project. (PART 1)

Entity responsible of the development: Universitat Politècnica de València.

Supervisor: Prof. Dr. Rafael Miró Herrero

Authors/Research staff: Patricio Hidalga García-Bermejo

Financing Entity: Kernkraftwerk Leibstadt, AG.

Submittal Date: $16 / 10 / 2018$ 


\section{REFERENCES}

Abarca, A., Miró, R., Verdú, G., \& Bermejo, J. A. (2016). Analysis of thermal-hydraulic fluctuations in Trillo NPP with CTF/PARCS v. 2.7 coupled code. Journal of Nuclear Engineering and Radiation Science, 2(2), 1-5. https://doi.org/10.1115/1.4031660

Avramova, M., \& Ivanov, K. (2015). CTF - A Thermal-Hydraulic Subchannel Code for LWRs Transient Analyses User's Manual.

Bajorek, S. (2011). TRACE V5.435 Theory Manual: Filed Equations, Solution Methods, and Physical Models. Technical report.

Barrachina, T., Garcia-Fenoll, M., Ánchel, F., Miró, R., Verdú, G., Pereira, C., da Silva, C., Ortego, A., \& Martínez-Murillo, J. C. (2011). REA 3D-dynamic analysis in Almaraz NPP with RELAP5/PARCS v2.7 and SIMTAB cross-sections tables. Progress in Nuclear Energy, 53(8), 1167-1180. https://doi.org/https://doi.org/10.1016/j.pnucene.2011.07.012

Beard, C., Mitchell, D., \& Slagle, W. (2006). RIA LIMITS BASED ON COMMERCIAL PWR CORE RESPONSE TO RIA.

Bestion, D., \& Guelfi, A. (2005). Multiscale analysis of nuclear reactors thermal-hydraulics the NEPTUNE project. Http://Dx.Doi.Org/10.1051/Lhb:200505006. https://doi.org/10.1051/lhb:200505006

Blyth, T., Porter, N., Avramova, M., \& Ivanov, K. (2013). BENCHMARK FOR UNCERTAINTY ANALYSIS IN MODELLING (UAM) FOR DESIGN, OPERATION AND SAFETY ANALYSIS OF LWRs. https://www.oecd-nea.org/science/docs/2013/nscdoc2013-7.pdf

Boslaugh, S. (2012). Statistics in a Nutshell: A Desktop Quick Reference. O'Reilly Media. https://books.google.ch/books?id=Fc4fBgAAQBAJ 
Development and validation of a multi-scale and multi-physics methodology for the safety analysis of fast transients in Light Water Reactors

Boyac, B., Duffey, R., Griffith, P., Lellouche, G., Levy, S., Rohatgi, G., Wilson, G., Wulff, W., \& Zuber, N. (1989). Application of Code Scaling, Applicability and Uncertainty Evaluation Methodology to a Large-Break Loss-of-Coolant Accident. https://www.nrc.gov/docs/ML0303/ML030380473.pdf

Brun, W., Keren, G., Kirkeboen, G., \& Montgomery, H. (2011). Perspectives on thinking, judging, and decision making. Universitetsforlaget. http://urn.kb.se/resolve?urn=urn:nbn:se:su:diva-70546

Butkovich, T. R. (n.d.). Mechanical and thermomechanical calculations related to the storage of spent nuclear-fuel assemblies in granite. https://doi.org/10.2172/59225

CTF User's Group. (2018). Fifth COBRA-TF (CTF) User's Group Meeting - CTF-5. https://www.ne.ncsu.edu/wp-content/uploads/2018/05/CTF-5-Announcement-final.pdf

D’Auria, F., \& Salah, A. (2006). Methodologies for Qualification and Application of Computational Tools for Accident Analysis in Nuclear Power Plants.

D’Auria, F., Salah, A., Petruzzi, A., \& Del Nevo, A. (2006). State of the art in using best estimate calculation tools in nuclear technology. Nuclear Engineering and Technology, 38 .

de Rocquigny, E., Devictor, N., \& Tarantola, S. (2008). Uncertainty in Industrial Practice: A Guide to Quantitative Uncertainty Management. Wiley. https://books.google.ch/books?id=qg8bqw6ByskC

Demazière, C. (2019). Modelling of Nuclear Reactor Multi-physics: From Local Balance Equations to Macroscopic Models in Neutronics and Thermal-Hydraulics. Elsevier Science $\backslash \&$ Technology. https://books.google.ch/books?id=Hat9xAEACAAJ

Downar, T., Xu, Y., \& Seker, V. (2012). PARCS v3.0 U.S. NRC Core Neutronics Simulator. Theory Manual, University of Michigan.

Energy Innovation Hub. (2010). CASL-Consortium for Advanced Simulation of Light Water Reactors. http://www.casl.gov/about-casl.shtml

ENSI. (2018). Richtlinie für die schweizerischen Kernanlagen. ENSI-A01: Technische Sicherheitsanalyse für bestehende Kernanlagen: Umfang, Methodik und Randbedingungen.

ENSI. (2019). Richtlinie für die schweizerischen Kernanlagen. ENSI-G02: Auslegungsgrundsätze für in Betrieb stehende Kernkraftwerke.

Ferroukhi, H., Zerkak, O., \& Chawla, R. (2009). Cross-section modelling effects on pressurised water reactor main steam line break analyses. Annals of Nuclear Energy, 36(8), 1184-1200. https://doi.org/https://doi.org/10.1016/j.anucene.2009.04.006

Forthofer, R. N., \& Lee, E. S. (1995). Introduction to Biostatistics: A Guide to Design, Analysis, and Discovery. Academic Press. https://books.google.ch/books?id=xCVrAAAAMAAJ 

Reactors.

Funtowicz, S. O., \& Ravetz, J. R. (1990). Uncertainty and Quality in Science for Policy. Springer Netherlands. https://books.google.ch/books?id=lINAsNfN7i4C

Geelhood, K. J., Luscher, W. G., \& Beyer, C. E. (2011). FRAPCON-3.4: A Computer Code for the Calculation of Steady-State, Thermal-Mechanical Behavior of Oxide Fuel Rods for High Burnup.

Geelhood, K. J., Luscher, W. G., Beyer, C. E., \& Cuta, J. M. (2011). FRAPTRAN 1.4: A Computer Code for the Transient Analysis of Oxide Fuel Rods.

Giust, F., Grimm, P., \& Chawla, R. (2010). Experimental validation of radial reconstructed pin-power distributions in full-scale BWR fuel assemblies with and without control blade. Annals of Nuclear Energy, 37(12), 1629-1639. https://doi.org/https://doi.org/10.1016/j.anucene.2010.08.006

Glaeser, H. (2008). GRS Method for Uncertainty and Sensitivity Evaluation of Code Results and Applications. Science and Technology of Nuclear Installations, 2008. https://doi.org/10.1155/2008/798901

GRS. (2019). ATHLET 3.2 Program Overview.

Guba, A., Makai, M., \& Pál, L. (2003). Statistical aspects of best estimate method-I. Reliability Engineering \& System Safety, 80(3), 217-232. https://doi.org/https://doi.org/10.1016/S0951-8320(03)00022-X

Hébert, A. (1987). Trivac: A Modular Diffusion Code for Fuel Management and Design Applications. Nuclear Journal of Canada, 1, 325.

Hidalga, P. (2018). UNCERTAINTY AND SENSITIVITY ANALYSIS IN VOID FRACTION PREDIC-TION WITH CTF-UPVIS OF THE PSBT AND BFBT FUEL MODELS FOR TRAN-SIENT CASES. ANS Best Estimate Plus Uncertainty International Conference.

Hidalga, Patricio, Abarca, A., Miró, R., Sekrhi, A., \& Verdú, G. (2019a). A multi-scale and multi-physics simulation methodology with the state-of-the-art tools for safety analysis in light water reactors applied to a turbine trip scenario (PART I). Nuclear Engineering and Design, 350, 195-204. https://doi.org/https://doi.org/10.1016/j.nucengdes.2019.05.008

Hidalga, Patricio, Abarca, A., Miró, R., Sekrhi, A., \& Verdú, G. (2019b). A multi-scale and multi-physics simulation methodology with the state-of-the-art tools for safety analysis in Light Water Reactors applied to a Turbine Trip scenario (Part II). Nuclear Engineering and Design, 350, 205-213. https://doi.org/https://doi.org/10.1016/j.nucengdes.2019.05.009

Hong, I., \& Connolly, A. (2008). Generalized Tolerance Limit Evaluation Method to Determine Statistically Meaningful Minimum Code Simulations (Vol. 4). https://doi.org/10.1115/ICONE16-48448 
Development and validation of a multi-scale and multi-physics methodology for the safety analysis of fast transients in Light Water Reactors

Hong, I. S., Oh, D. Y., \& Kim, I. G. (n.d.). Generic Application of Wilks' Tolerance Limit Evaluation Approach to Nuclear http://inis.iaea.org/search/search.aspx?orig_q=RN:45107564

IAEA. (1992). Probabilistic Safety Assessment (Issue 6). INTERNATIONAL ATOMIC ENERGY AGENCY. https://www.iaea.org/publications/3789/probabilistic-safetyassessment

IAEA NUCLEAR ENERGY SERIES. (2009). Deterministic Safety Analysis for Nuclear Power Plants.

IAEA NUCLEAR ENERGY SERIES. (2015). Plant Life Management Models For Long Term Operation Of Nuclear Power Plants No. NP-T-3.18. https://wwwpub.iaea.org/MTCD/Publications/PDF/Pub1655_web.pdf

IAEA Safety Report Series. (2008). Best Estimate Safety Analysis for Nuclear Power Plants: Uncertainty Evaluation.

IAEA SAFETY STANDARDS SERIES. (2006). FUNDAMENTAL SAFETY PRINCIPLES.

International Nuclear Safety Advisory Group INSAG-10. (1996). Defense in depth in Nuclear Safety.

Ivanov, K. N., Macian-Juan, R., Irani, A., \& Baratta, A. J. (1999). Features and performance of a coupled three-dimensional thermal-hydraulic/kinetics TRAC-PF1/NEM pressurized water reactor (PWR) analysis code. Annals of Nuclear Energy, 26(15), 1407-1417. https://doi.org/https://doi.org/10.1016/S0306-4549(99)00017-1

Jenks, R. P., \& Martinez, V. (1988). TRAC (Transient Reactor Analysis Code) support software.

KAERI. (2009). ATLAS Facility Rescription Report. https://inis.iaea.org/collection/NCLCollectionStore/_Public/41/070/41070429.pdf

Kahler, A. C., MacFarlane, R. E., Mosteller, R. D., Kiedrowski, B. C., Frankle, S. C., Chadwick, M. B., McKnight, R. D., Lell, R. M., Palmiotti, G., Hiruta, H., Herman, M., Arcilla, R., Mughabghab, S. F., Sublet, J. C., Trkov, A., Trumbull, T. H., \& Dunn, M. (2011). ENDF/B-VII.1 Neutron Cross Section Data Testing with Critical Assembly Benchmarks and Reactor Experiments. Nuclear Data Sheets, 112(12), 2997-3036. https://doi.org/https://doi.org/10.1016/j.nds.2011.11.003

Khuri, A. I., \& Mukhopadhyay, S. (2010). Response surface methodology. WIREs Computational Statistics , 2(2), 128-149. https://doi.org/10.1002/wics.73

Lahey, R. T., \& Moody, F. J. (1993). The thermal-hydraulics of a boiling water nuclear reactor. American Nuclear Society. https://books.google.ch/books?id=dXBTAAAAMAAJ

Langenbuch, S. (1984). QUABOX/CUBBOX-HYCA, Ein dreidimensionales Kernmodell mit parallelen Kuhlkanalen fur Leichtwasserreaktore. 

collections/nuregs/agreement/ia0407/\#pub-info

Martin, R. P., \& O'Dell, L. D. (2005). AREVA's realistic large break LOCA analysis methodology. Nuclear Engineering and Design, 235(16), 1713-1725. https://doi.org/10.1016/J.NUCENGDES.2005.02.004

Martorell Aigües, P. (2019). Aportaciones al modelado de la indisponibilidad de componentes y la fiabilidad humana para la mejora de la seguridad de las centrales nucleares informada en el riesgo [Universitat Politècnica de València]. https://doi.org/10.4995/Thesis/10251/118794

MATLAB and Statistics Toolbox Release 2017a. (2017).

Michel, B., Sercombe, J., \& Thouvenin, G. (2008). A new phenomenological criterion for pellet-cladding interaction rupture. Nuclear Engineering and Design, 238(7), 16121628. https://doi.org/https://doi.org/10.1016/j.nucengdes.2008.01.012

Miro, R., Jambrina, A., Mesado, C., Barrachina, T., \& Verdú, G. (2016). TRAC-BF1 to TRACE Model Semi-Automatic Conversion. PBTT Example.

National Technology and Engineering Solutions of Sandia. (2017). DAKOTA Explore and predict with confidence.

NEA. (2009). Nuclear Fuel Behaviour in Loss-of-coolant Accident (LOCA) Conditions. https://doi.org/ISBN 978-92-64-99091-3

Odar, F. (2000). Software Quality Assurance Procedures for NRC Thermal Hydraulic Codes (NUREG-1737). https://www.nrc.gov/docs/ML0101/ML010170081.pdf

OECD/NEA. (2016). International Benchmark on Pressurized Water Reactors Sub-channel and Bundle Tests (Vol. 2).

Papadopoulos, P., \& Sekrhi, A. (2017). Development of a Coupled TRACE/PARCS Model for KKL and Benchmark Against the Turbine Trip Test. https://www.nrc.gov/docs/ML1709/ML17096A273.pdf

Paulsen, M. P. (2014). RETRAN-3D - A Program for Transient Thermal-Hydraulic Analysis of Complex Fluid Flow Systems Volume 5: Modeling Guidelines.

Pavlovski, O. A., \& Ilyn, L. A. (1987). Radiological consequences of the Chernobyl accident in the Soviet Union and measures taken to mitigate their impact. https://www.iaea.org/sites/default/files/publications/magazines/bulletin/bull294/29402791724.pdf

Ponciroli, R., Wang, Y., Zhou, Z., Botterud, A., Jenkins, J., Vilim, R. B., \& Ganda, F. (2017). Profitability Evaluation of Load-Following Nuclear Units with Physics-Induced Operational Constraints. Nuclear Technology, 200(3), 189-207. https://doi.org/10.1080/00295450.2017.1388668

Pusa, M., \& Isotalo, A. (2017). Uncertainty analysis of assembly and core-level calculations 
Development and validation of a multi-scale and multi-physics methodology for the safety analysis of fast transients in Light Water Reactors

with application to CASMO-4E and SIMULATE-3. Annals of Nuclear Energy, 104, 124-131. https://doi.org/https://doi.org/10.1016/j.anucene.2017.01.042

Rao, K. R. (Ed.). (2009). Companion Guide to the ASME Boiler and Pressure Vessel Code, Volume 3, Third Edition. ASME Press. https://doi.org/10.1115/1.802717

Robert, M., Farvacque, M., Parent, M., \& Faydide, B. (2003). CATHARE 2 V2.5 : a fully validated CATHARE version for various applications.

Rohde, U., Kliem, S., Grundmann, U., Baier, S., Bilodid, Y., Duerigen, S., Fridman, E., Gommlich, A., Grahn, A., Holt, L., Kozmenkov, Y., \& Mittag, S. (2016). The reactor dynamics code DYN3D - models, validation and applications. Progress in Nuclear Energy, 89, 170-190. https://doi.org/https://doi.org/10.1016/j.pnucene.2016.02.013

Rotmans, J., \& van Asselt, M. B. A. (2001). Uncertainty in Integrated Assessment Modelling: A Labyrinthic Path. Integrated Assessment, 2(2), 43-55. https://doi.org/10.1023/A:1011588816469

Rubin, A. (2010). OECD/NRC Benchmark Based on NUPEC PWR Sub-channel and Bundle Tests (PSBT), Volume I: Experimental Database and Final Problem Specifications.

Rudling, P., Jernkvist, L. O., Garzarolli, F., Adamson, R., Mahmood, T., Strasser, A., \& Patterson, C. (2016). Nuclear fuel behaviour under ria conditions. Advanced Nuclear Technology International, Mölnlycke.

Saltelli, A. (2002). Making best use of model evaluations to compute sensitivity indices. Computer Physics Communications, 145(2), 280-297. https://doi.org/https://doi.org/10.1016/S0010-4655(02)00280-1

Schultz, R. (2003). RELAP5-3D@ Code Manual Volume V: User's Guidelines.

Siu, N., Stutzke, M., Dennis, S., \& Harrison, D. (2016). Probabilistic Risk Assessment and Regulatory Decisionmaking. https://www.nrc.gov/docs/ML1624/ML16245A032.pdf

Smith, K. (1985). QPANDA An Advanced Nodal Method for LWR Analyses.

Sobol, M. I. (1993). Sensitivity Analysis for Non-Linear Mathematical Models. Math. Modeling Comput. Exp., 1, 407-414. http://ci.nii.ac.jp/naid/10030641711/en/

Stacey, W M. (2018). Nuclear Reactor Physics. Wiley. https://books.google.ch/books?id=NzIJDwAAQBAJ

Stacey, Weston M. (1972). Variational Flux Synthesis Methods for Multigroup Neutron Diffusion Theory. Nuclear Science and Engineering, 47(4), 449-469. https://doi.org/10.13182/NSE72-A22436

Turinsky, P. J. (1994). NESTLE: Few-group Neutron Diffusion Equation Solver Utilizing the Nodal Expan-sion Method for Eigenvalue, Adjoint, Fixed-Source Steady-State and Transient Problems.

USNRC. (2007a). Chapter 15 Standard Review Plan for the Review of Safety Analysis Reports 

https://www.nrc.gov/docs/ML0707/ML070710376.pdf

USNRC. (2007b). NUREG-0800, Chapter 15.2.1 Standard Review Plan for the Loss of external load; Turbine trip; loss of condenser vacuum; closure of main steam isolation valve (BWR); and steam pressure regulator failure (PWR). https://www.nrc.gov/docs/ML0703/ML070300702.pdf

USNRC. (2007c). NUREG-0800, Chapter 15 Standard Review Plan for the Review of Safety Analysis Reports for Nuclear Power Plants: LWR Edition - Transient and Accident Analysis. https://www.casl.gov/sites/default/files/docs/CASL-U-2015-0055-000.pdf

USNRC. (2017a). Appendix A to Part 50 - General Design Criteria for Nuclear Power Plants. https://www.nrc.gov/reading-rm/doc-collections/cfr/part050/part050-appa.html

USNRC. (2017b). Appendix $K$ to Part 50-ECCS Evaluation Models. https://www.nrc.gov/reading-rm/doc-collections/cfr/part050/part050-appk.html

USNRC. (2017c). Code of Federal Regulations 10 CFR 50.46: Acceptance criteria for emergency core cooling systems for light-water nuclear power reactors. https://www.nrc.gov/reading-rm/doc-collections/cfr/part050/part050-0046.html

USNRC. (2019). Appendix $G$ to Part 50-Fracture Toughness Requirements. https://www.nrc.gov/reading-rm/doc-collections/cfr/part050/part050-appg.html

Vedovi, J., Yang, J., Klebanov, L., Vreeland, D. G., \& Zino, J. F. (2012). Best-estimate plus uncertainty thermal-hydraulic stability analysis of BWRs using TRACG code. American Nuclear Society - ANS. http://inis.iaea.org/search/search.aspx?orig_q=RN:44065603

Verdú, G., Muñoz-Cobo, J. L., Pereira, C., \& Ginestar, D. (1993). Lambda modes of the neutron-diffusion equation: Application to B.W.R.'s out-of-phase instabilities. Annals of Nuclear Energy, 20(7), 477-501. https://doi.org/https://doi.org/10.1016/03064549(93)90089-8

Versluis, R. M., Huff, D., Cook, T. L., \& Johnson, R. S. (2013). NEAMS: Software Verification and Validation Plan Requirements. https://www.hsdl.org/?view\&did=744485

Wall, I. B. (1980). Probabilistic risk assessment in nuclear power plant regulation. Nuclear Engineering and Design, 60(1), 11-24. https://doi.org/https://doi.org/10.1016/00295493(80)90248-4

Ward, A., Xu, Y., \& Downar, T. (2016). GenPMAXS-v6.2 Code for Generating the PARCS Cross Section Interface File PMAXS.

Wieselquist, W., Williams, M., Wiarda, D., Pigni, M., \& Mertyurek, U. (2018). Overview of Nuclear Data Uncertainty in Scale and Application to Light Water Reactor Uncertainty Analysis (NUREG/CR-7249, ORNL/TM-2017/706). https://www.nrc.gov/reading$\mathrm{rm} /$ doc-collections/nuregs/contract/cr7249/

Wilks, S. S. (1941). Determination of Sample Sizes for Setting Tolerance Limits. The Annals of Mathematical Statistics, 12(1), 91-96. http://www.jstor.org/stable/2235627 